\title{
Elliptic Double Affine Hecke Algebras
}

Eric M. RAINS

Department of Mathematics, California Institute of Technology, USA

E-mail: rains@caltech.edu

Received December 19, 2019, in final form October 16, 2020; Published online November 05, 2020

https://doi.org/10.3842/SIGMA.2020.111

\begin{abstract}
We give a construction of an affine Hecke algebra associated to any Coxeter group acting on an abelian variety by reflections; in the case of an affine Weyl group, the result is an elliptic analogue of the usual double affine Hecke algebra. As an application, we use a variant of the $\tilde{C}_{n}$ version of the construction to construct a flat noncommutative deformation of the $n$th symmetric power of any rational surface with a smooth anticanonical curve, and give a further construction which conjecturally is a corresponding deformation of the Hilbert scheme of points.
\end{abstract}

Key words: elliptic curves; Hecke algebras; noncommutative deformations

2020 Mathematics Subject Classification: 33D80; 39A70; 14A22

\section{Contents}

1 Introduction 1

2 Line bundles on $E^{n}$ and their sections $\quad 8$

3 Coxeter group actions on abelian varieties $\quad 26$

4 Elliptic analogues of affine Hecke algebras $\quad 34$

$\begin{array}{lll}5 & \text { Infinite groups } & 60\end{array}$

$\begin{array}{lll}6 & \text { The (double) affine case } & 76\end{array}$

$\begin{array}{llr}7 & \text { The } C^{\vee} C_{n} \text { case } & 87\end{array}$

8 The (spherical) $C^{\vee} C_{n}$ Fourier transform $\quad 107$

9 Deformations of Hilbert schemes $\quad 122$

$\begin{array}{ll}\text { References } & 131\end{array}$

\section{Introduction}

The origin of this paper was a question of P. Etingof which was conveyed to the author by A. Okounkov at a 2011 conference, ${ }^{1}$ to wit whether the author knew of a way to construct

This paper is a contribution to the Special Issue on Elliptic Integrable Systems, Special Functions and Quantum Field Theory. The full collection is available at https://www.emis.de/journals/SIGMA/elliptic-integrablesystems.html

${ }^{1}$ Affine Hecke Algebras, the Langlands Program, Conformal Field Theory and Super Yang-Mills Theory, Centre International de Rencontres Mathématiques (Luminy), June 2011. 
noncommutative deformations of symmetric powers of the complement of a smooth cubic plane curve. Although the answer was "no" (at the time, see below!), it seemed likely that it should be possible to extend the approach of [28] (which the author and S. Ruijsenaars had developed earlier that month) to multivariate difference operators; although this would not answer the question as posed, it would give analogous deformations associated to the complement of a smooth biquadratic curve in $\mathbb{P}^{1} \times \mathbb{P}^{1}$, represented as algebras of elliptic difference operators in $n$ variables. The author continued to develop this approach while on a sabbatical that fall at MIT, eventually coming up with a construction for such deformations for any rational surface equipped with a smooth anticanonical curve and a rational ruling.

There were, however, a couple of significant issues. One was that the spaces of operators were cut out by a number of conditions, including in particular certain residue conditions that only made sense for generic values of the parameters. This would have been merely an annoying technicality, except that the conditions specifically failed to make sense in the commutative case, making it rather difficult to consider the family as actually being a deformation. This could be worked around by considering the family as a whole, or in other words only considering those operators that could be extended to an open subset of parameter space. However, although this would indeed give a well-defined family of algebras, it would make the question of flatness even more difficult, and would in principle even allow the representation in difference operators to fail to be faithful!

Despite these difficulties, the definition was still well-behaved enough to allow a fair amount of experimentation. One thing that became clear was that the construction directly led to some spaces of operators that had been considered in the literature, in particular those associated to the difference equations for interpolation and biorthogonal functions [23, 24], of particular interest in the latter case since no satisfying construction was yet known for the full space of operators. In addition, since these functions degenerate to more familiar functions, to wit the Macdonald and Koornwinder polynomials, this suggested that the algebras of elliptic difference operators should degenerate to algebras related to those polynomials. In particular, the latter algebras can be constructed as spherical algebras of appropriate double affine Hecke algebras, and P. Etingof suggested to the author that the same might hold at the elliptic level, and give a possible approach to flatness.

Indeed, the same approach to constructing the algebra of operators (as operators preserving (locally) holomorphic functions and satisfying appropriate vanishing conditions on the coefficients) could be fairly easily extended to give a construction of elliptic double affine Hecke algebras. The resulting residue conditions turned out to be essentially those of [11], with again the caveat that they only make sense when the noncommutative parameter $q$ is non-torsion. In fact, something slightly stronger is true: the residue conditions are well-behaved as long as one only considers a sufficiently small interval relative to an appropriate (Bruhat) filtration, with the constraint on the interval being simply that it act faithfully. This led the author to investigate that filtration more carefully, leading eventually to the realization that (a) the residue conditions always make sense on rank 1 subalgebras (which are very special cases of the construction of [11]), and (b) those rank 1 subalgebras always generate a flat algebra, even when $q$ is torsion. As a result, one could avoid the residue conditions entirely and simply consider the algebra generated by the rank 1 algebras. It is then relatively straightforward to show that the resulting family of algebras is flat (and the representation as difference-reflection operators is faithful), and not too difficult to show that this flatness is inherited by the spherical algebra. Moreover, much of the theory can be developed for quite general actions of Coxeter groups on abelian varieties, so that the DAHAs are just the special cases in which the Coxeter group is affine.

In the above discussion, we have neglected a few technical issues. The first is that the deformations of symmetric powers are not, in general, algebras of operators. The difficulty is that, with the exception of complements of smooth anticanonical curves in del Pezzo surfaces, 
none of the surfaces we wish to deform are actually affine! Since they are only quasiprojective in general, it is easier to simply deform the symmetric power of the original projective surface (and then take the appropriate localization if desired). This still requires a choice of ample divisor, and one then encounters the difficulty that twisting a noncommutative variety by a line bundle tends to change the noncommutative variety. As a result, the object actually being deformed is the category of line bundles on the symmetric power.

When it comes to the DAHA, however, the situation is even more complicated. The problem is that the elliptic DAHA essentially arises by replacing one of the two commutative subalgebras of the usual DAHA by the structure sheaf of an abelian variety of the form $E^{n}$. In the affine case considered in [11], the commutative subalgebra is finite over the center of the affine Hecke algebra, and thus one is naturally led to consider sheaves over the center, or in other words sheaves on the quotient $E^{n} / W$, where $W$ is the relevant finite Weyl group. Unfortunately, in the double affine setting, $W$ is replaced by an affine Weyl group, and there is no such quotient scheme! As a result, the trickiest part of our construction turns out to be simply figuring out what kind of object we should be constructing. The key idea, coming from earlier work in noncommutative geometry $[2,37]$ is that since the elliptic DAHA should have $\mathcal{O}_{E^{n}}$ as a subalgebra, it should have a natural bimodule structure over $\mathcal{O}_{E^{n}}$, and thus correspond to a quasicoherent sheaf on the product $E^{n} \times E^{n}$. Subject to some finiteness conditions (satisfied for any sheaf of meromorphic difference operators), such bimodules form a monoidal category, and thus we can construct the elliptic DAHA as a monoid object ("sheaf algebra") in that category.

A final technical issue is that we wish to deform symmetric powers of arbitrary blowups of $\mathbb{P}^{1} \times \mathbb{P}^{1}$ or the Hirzebruch surface $F_{1}$. Each time we blow up a point, we acquire a new parameter, and thus our construction needs to admit an unbounded number of parameters. This is an issue from the standpoint of traditional double affine Hecke algebras, where one normally has precisely one parameter per root (which must be constant on orbits), plus an overall parameter $q$. One partial exception is the $C^{\vee} C_{n}$ case, where one has a total of $5+1$ parameters. This is normally explained by taking a nonreduced root system, so that one has 5 orbits of roots, but from the standpoint of the actual algebra, this is rather artificial: there is an action of $S_{2} \times S_{2}$ on the parameters that has no effect on the algebra, but moves degrees of freedom between corresponding short and long roots. It is thus much more natural to view those four parameters as assigning an unordered pair to each orbit of short roots of the reduced root system. This turns out to generalize easily to the elliptic setting: we obtain an elliptic DAHA for every assignment of an effective divisor on $E$ to each orbit of roots. This causes some difficulties in constructing the spherical algebra, as the usual construction via idempotents fails even generically, but one can show that the spherical algebra still continues to inherit flatness in this general case.

As we mentioned above, the construction of deformations of $\operatorname{Sym}^{n}(X)$, where $X$ is a projective rational surface with a choice of smooth anticanonical curve, depends on a choice of rational ruling on $X$. One consequence is that we cannot directly obtain the case $X=\mathbb{P}^{2}$ from our construction. This can be worked around by blowing up a point but then only considering those line bundles coming from $\mathbb{P}^{2}$, but this approach leads to a nontrivial question of showing that the result is independent of the choice of point. Similarly, if $X \cong \mathbb{P}^{1} \times \mathbb{P}^{1}$, then there are two choices of ruling on $X$, but deformation theory suggests that the resulting deformations should be the same (both have the maximum number of parameters). Both questions turn out to reduce to the existence of a certain generalized Fourier transform in the $\mathbb{P}^{1} \times \mathbb{P}^{1}$ case, which is also key to proving the most general form of the flatness result. (The DAHA only tells us that certain sheaves are flat, so gives only an asymptotic flatness result for their global sections.) We find that not only is our deformation of the category of symmetric powers of line bundles on $X$ flat (modulo some possibility of bad parameters in codimension $\geq 2$ not including the original symmetric-power-of-commutative-surface case), but it is invariant under the action of a Coxeter group of type $W\left(E_{m+1}\right)$. (In other words, to first approximation, the 
construction only depends on the underlying surface $X$ and two points $q$ and $t$ of the Jacobian of the anticanonical curve.) Note that both facts are actually not true for the full original family of commutative categories, but hold for the subcategory in which we only allow those morphisms that extend to a neighborhood in the family.

The plan of this paper is as follows. First, in Section 2, we deal with a largely notational issue that arises due to the fact that we wish to deal with the construction from a purely algebraic standpoint. The issue is that we need in many cases to deal with twisted versions of our algebras, in which the coefficients of the operators lie in nontrivial line bundles. To make sense of this, one must not only describe those line bundles but various maps between tensor products of pullbacks of those bundles through elements of the Coxeter group, with associated concerns about compatibility. In the analytic setting, one can avoid most of those issues by constructing the line bundle via an appropriate automorphy factor on the universal cover, and our objective in Section 2 is to do something similar in the algebraic setting. The key idea is to replace the individual curve $E$ by the universal curve over the moduli stack of elliptic curves; it turns out that one can compute the Picard group of the corresponding stack $\mathcal{E}^{n}$ in general, with only very mild rigidification required to make pullbacks and tensor products behave well. In addition, certain of the line bundles come with natural global sections, which one can use to construct sections of more general bundles; our most significant result along these lines gives conditions for a function on the analytic locus described via theta functions to extend to the full moduli stack (i.e., when it extends in a nice way to elliptic curves over an arbitrary base). We also partially consider the case of varieties which are isogenous to powers of elliptic curves (i.e., over a moduli stack of elliptic curves with a cyclic subgroup), and as an application give some results on spaces of invariant sections of equivariant line bundles on $\mathcal{E}^{n}$. The main result along those lines states that the dimension of the space of invariants is independent of the curve $E$ with only finitely many possible exceptions (supersingular curves of characteristic dividing the order of the group).

In Section 3, we give some structural results on the main scenario we consider in the sequel, namely a Coxeter group acting on an abelian variety "by reflections". In particular, we show that under reasonable conditions every root of the Coxeter group can be assigned an associated "coroot" morphism to an elliptic curve, compatibly with the linear relations between roots in the standard reflection representation (and satisfying suitable notions of positivity!). This is a key ingredient in our construction, as our parameters will correspond to effective divisors on those curves. We also show that in the case of a finite Coxeter group, the invariant theory is better behaved than suggested by the results of Section 2: as long as a certain isogeny (which is an isomorphism in the most natural cases) has diagonalizable kernel, the invariant theory continues to behave well even for supersingular curves. This flatness of invariants is a crucial ingredient in proving flatness of the spherical algebra, and in particular means the $C^{\vee} C_{n}$ case will be flat over any field.

Section 4 is largely a recapitulation of the construction of [11], in which we associate to any finite Coxeter group $W$ acting on a family $X$ of abelian varieties a family (the "elliptic Hecke algebra" for concision, though it should be thought of as affine, with the role of the commutative subalgebra being played by the structure sheaf $\mathcal{O}_{X}$ ) of sheaves of algebras on $X / W$ parametrized by effective divisors on the "coroot" curves. The main difference, apart from allowing arbitrary numbers of parameters and slightly more general abelian varieties, is that we replace the residue conditions of [11] by the (equivalent) condition that the operators preserve the spaces of local sections of the structure sheaf of $X$ on $W$-invariant open subsets, as the latter is easier to generalize from a conceptual standpoint. Our main new tool for studying these algebras is a natural filtration by Bruhat order on $W$, which allows us to express various subsheaves as extensions of line bundles in natural ways. In particular, this makes it easy to show that the algebra is generated by the subalgebras corresponding to simple roots, which will be a key ingredient of the extension to infinite Coxeter groups, as well as giving a construction 
which is easily seen to respect base change. We also prove an important technical lemma on the space of "invariants" of a module over the elliptic AHA, giving fairly general conditions on a family of modules guaranteeing that the invariants are well-behaved; this will be the key lemma in proving flatness of spherical algebras. In addition, we give an analogue of Mackey's theorem for the case of parabolic subgroups in which the usual sum over double cosets is replaced by a filtration.

Section 5 begins with a discussion of sheaf algebras, which we use in place of a sheaf of algebras on the quotient. Since the corresponding tensor product of bimodules is somewhat tricky to deal with, we discuss approaches to dealing with this issue (and in particular ways to describe the maps $A \otimes B \rightarrow C$ we need in order to discuss algebras or categories). This makes it relatively straightforward to construct analogues of the affine Hecke algebra in which $W$ is replaced by an infinite Coxeter group: start with the sheaf algebra of meromorphic reflection operators and take the sheaf subalgebra generated by the rank 1 Hecke algebras. (The only tricky aspect comes when we consider twisted forms of the algebra, for which we prove a fairly general result about "orders" in twisted forms of $k(X)[W]$ containing $\mathcal{O}_{X}[W]$.) Most of the results extend immediately from the finite case (with the caveat that any parabolic subgroups considered should be finite); in particular, the infinite analogue of the Mackey result is precisely the remaining result we need to show that the spherical algebras are flat.

Section 6 discusses the special case in which $W$ is an affine Weyl group, so that the sheaf algebras constructed in Section 5 are analogues of double affine Hecke algebras. Apart from some mild issues about viewing $q$ as a parameter (not the case for the standard construction), this mainly consists of some observations about the spherical algebra (relative to the associated finite Weyl group): the fact that the fibers are (sheaf) algebras of difference operators, and are thus in a natural sense domains, and the fact that the elliptic DAHA is at least generically Morita equivalent to its spherical algebra (as well as versions in which some of the parameters have been shifted by $q$ ). In addition, we discuss the consequences of the fact that the action of $\widetilde{W}$ fails to be faithful when $q$ is torsion: not only does the sheaf algebra come from a sheaf of algebras on the quotient by the image of $\widetilde{W}$, but it has a $2 n$-dimensional center (over which it is presumably finite). Moreover, under mild conditions on the twisting, we can identify the center as the spherical algebra of an elliptic DAHA with $q=0$ living on an isogenous abelian variety.

Section 7 considers in detail the case that $W$ is the affine Weyl group of type $C_{n}$ and $X$ is a particularly nice action of that group, with a view to constructing deformations of symmetric powers of rational surfaces. In addition to the spherical algebras themselves, one must also consider certain intertwining bimodules. We prove that these are always flat as sheaf bimodules, and show that in the case $t=0$ the result is indeed a symmetric power of the univariate case (which was constructed in [26] without reference to DAHAs). This enables us to at least partially extend the flatness as sheaves to flatness of global sections, by giving a number of cases in which the sheaves are acyclic. In addition to these general results, our main result in this section is showing that if we blow up 8 points of $\mathbb{P}^{1} \times \mathbb{P}^{1}$, then there is a hypersurface in parameter space on which the "anticanonical" algebra is an integrable system: it is generated by $n+1$ commuting (and self-adjoint) elliptic difference operators in $n$ variables. (One can verify that this is precisely the integrable system of $[6,7,16]$. In addition, the geometry strongly suggests the existence of other integrable systems with the same number of parameters, but higher-order operators.)

Section 8 deals with the question of showing that the construction is mostly independent of the way in which we represented our rational surface as a blowup of a ruled surface. The key ingredient is a certain "Fourier transform". Analytically, this should be represented by the integral operator with kernel constructed in [27], but there are difficulties in showing this is well-defined in general (as well as showing that it respects the additional conditions associated to any points we have blown up). As a result, we construct the transform in several steps. First, 
we give a construction that is manifestly well-defined (and a homomorphism) as long as $q$ is not torsion, but lives on a certain completion of the algebra of meromorphic difference operators. Although the construction is in general quite complicated, it is sufficiently well-behaved to allow us to compute a few special cases. The result in those special cases turns out to be well-defined even when $q$ is torsion, and this allows us to show that this formal transform extends in general. Moreover, the special cases we understand are sufficiently close to generating the full algebras that we can prove that the formal transform restricts to an actual transform. This also gives us some ability to explore how the algebras behave when we degenerate the elliptic curve, as it is easy to take limits of the almost-generators. In addition to allowing us to prove a much stronger flatness result, the Fourier transform also allows us to construct a large collection of "quasi-integrable" systems, in particular including the aforementioned operators associated to interpolation and biorthogonal functions.

Section 9 gives some partial results towards "desingularizing" the above construction; i.e., giving a deformation of the Hilbert scheme of points of $X$ rather than just the symmetric power. The basic idea is fairly straightforward: simply include additional bimodules that shift $t$ as well as the points being blown up. The argument for flatness breaks down in general, even at the level of sheaves, but we can show both flatness and agreement with Euler characteristics of line bundles on the Hilbert schemes in a fair number of cases. Moreover, for several of those cases, not only do we obtain the correct number of global sections, but one can identify those global sections as global sections on the Hilbert scheme in such a way that they satisfy precisely the same relations. In particular, this includes the line bundles corresponding to the embedding of $\operatorname{Hilb}^{n}(X)$ in a Grassmannian that takes the ideal sheaf $\mathcal{I}$ to the subspace $\Gamma(X ; \mathcal{I}(D)) \subset \Gamma\left(X ; \mathcal{O}_{X}(D)\right)$. (One can also show that the Fourier transform extends, so that again the construction essentially depends only on $X$ and not the particular way it was obtained from a Hirzebruch surface.)

We close with a summary of some of the various open problems that arose in the course of this work. (Of course, this is only a small sampling of such problems, as nearly any existing result on double affine Hecke algebras suggests the existence of a generalization to the elliptic case! We are also omitting some questions discussed in Sections 2 and 3, as they are peripheral to the main thrust of the work.) One big collection of questions has to do with the fact that, although we show that the spherical algebras of the elliptic DAHAs are flat in significant generality, we can prove almost nothing else about them in general. In particular, we cannot even show that they are Noetherian (even in the specific $C^{\vee} C_{n}$-type cases for which we have such strong flatness results). The approach to such questions in [1] suggests that one should be able to reduce this to the case in which everything is defined over a finite field, when one expects the spherical algebra to be finite over its center (which should itself be Noetherian); unfortunately, understanding the center (when $q$ is torsion) is itself an open problem. (This reduces to understanding the spherical algebra when $q=0$.)

Another natural question is whether the Fourier transform of the usual DAHA extends to the elliptic level. The construction of Section 8 can be viewed as a partial affirmative answer to this question in the $C^{\vee} C_{n}$ case, as it constructs a Fourier transform on the spherical algebra. For $n=1$, this at least implicitly leads to a Fourier transform on the elliptic DAHA by using the appropriate Morita equivalence, but even there it is unclear how to make the transformation explicit. (There is also a philosophical question: our work suggests that the transform should really be viewed as living on the spherical algebra, as the analogous transform on the DAHA is not as well behaved relative to the natural filtration.)

In fact, even in the $C_{n}$ case, there are still open questions about the Fourier transform, as the construction of Section 8 only applies to the case in which we have assigned precisely one parameter to the $D_{n}$-type roots. There is evidence suggesting the existence of some other Fourier transforms related to the versions of the DAHAs of types $A_{n}$ and $C_{n}$ that do not have this " $t$ " parameter. Indeed, the paper [35] discusses several integral transformations; one appears to 
relate two versions of the $A_{n}$ spherical algebra, while the other two appear to relate the $A_{n}$ spherical algebra to the $C_{n}$ spherical algebra. The main obstruction to understanding these cases is that the lack of a $t$ parameter makes it difficult to control the spaces of global sections, as we can no longer view the spherical algebras as deformations of a symmetric power. In any event, this suggests that the existence of isomorphisms between spherical algebras is a much more subtle question than one might have thought based on the classical theory of double affine Hecke algebras. Another source of questions about the Fourier transform is the analytic version constructed in [27]. In addition to the "interpolation kernel", that paper constructed a few other functions with some similar properties (the "Littlewood", "dual Littlewood" and "Kawanaka" kernels), and it is natural to ask whether those functions interact with the $C^{\vee} C_{n}$ spherical algebra in any interesting way. That such an interaction should exist is strongly suggested by the fact that the quadratic transforms proved in that paper were generalizations of results first proved using the action of affine Hecke algebras on Laurent polynomials.

There are also a number of questions about our deformations of symmetric powers having to do with taking global sections. One fundamental question has to do with the fact that our construction, although (mostly) flat and highly symmetrical, does not quite correspond directly to deformations of symmetric powers of surfaces: we must still make a choice of ample line bundle in order to obtain an actual projective deformation in the commutative case or a deformation of the category of sheaves in the noncommutative case. For $n=1$, it was shown in [26] that all such choices give the same result, but it will be difficult to extend those techniques to $n>1$. In addition, one would like to show that the corresponding family of commutative quasiprojective varieties is at least generically smooth (which experiment suggests is the case). Of course, we also expect that our conjectural deformation of Hilbert schemes should always be smooth (i.e., in the noncommutative case, that the corresponding category of coherent sheaves should satisfy Serre duality). In addition, one would like to have a proof that our family of algebras (or noncommutative varieties) actually depends on all of the parameters (and is not, say, simply a base change from a lower-dimensional family); this suggests trying to understand how the family relates to the infinitesimal deformation theory of the symmetric power. (Note, however, that the deformation associated to the $t$ parameter is almost certainly not locally trivial. Also, the fact that replacing $t$ by $q-t$ gives an isomorphic algebra implies that the corresponding Kodaira-Spencer map will vanish unless we first descend the family to the quotient by this symmetry, and it is unclear how to do so without breaking the representation via difference operators.) This would largely be settled if we could show that the $q=0$ case of the Hilbert scheme deformation agreed with the deformation constructed in [26] (as a moduli space of rank 1 sheaves on a noncommutative rational surface).

In any event, a choice of ample divisor allows one to translate each original line bundle into an actual sheaf on the (noncommutative) variety, and thus produces a saturated version of the original Hom space (by taking all morphisms between the sheaves). In the univariate case, the saturated morphisms were still difference operators, and there is a primarily combinatorial algorithm for computing the resulting dimensions. The question is more subtle in the multivariate setting, with two main issues arising. For some line bundles on $F_{1}$ (including those coming from line bundles on $\mathbb{P}^{2}$ ), we can only prove flatness away from a possible bad locus of codimension $\geq 2$, and some new approach to constructing global sections is likely needed to eliminate this possibility. The other tricky case arises from the elliptic pencil on a deformation of a non-Jacobian elliptic surface (i.e., in which the elliptic fibration does not have a section). There, not only do we want to know how many global sections there are (with the conjecture being that on the hypersurface where the algebra of global sections is nontrivial, it is flat), but also, by analogy with the Jacobian case, expect that the algebra of global sections will give a new integrable system, associated to the same parameters as the van Diejen/Komori-Hikami system, with the addition of a choice of nontrivial torsion point on the elliptic curve. 
Finally, the condition that a projective rational surface have a smooth anticanonical curve is quite restrictive, and in particular excludes a number of cases in which deformations were already known. Although the strong version of flatness cannot be expected to extend in general, one can still expect to have flatness for ample bundles. There are already issues before blowing up any points, as it appears one must give an analogue of the elliptic DAHA in which the elliptic curve becomes singular, reducible, or even nonreduced, and this can cause issues with the Bruhat filtration as well as the generation in rank 1. Beyond that, although it should be fairly straightforward (especially if the base curve remains integral) to consider blowups in smooth points of the base curve, this is again a pretty restrictive condition, while blowing up singular points quickly leads to a combinatorial explosion without some more conceptual approach.

\section{Line bundles on $E^{n}$ and their sections}

In the sequel, we will quite frequently need to specify line bundles on a power $E^{n}$ of an elliptic curve $E$ or (meromorphic) sections thereof. (Here $E$ will typically be defined over an algebraically closed field, though much of the discussion works over a general base.) At first glance, the problem of specifying a line bundle appears nearly trivial. Indeed, given any principally polarized abelian variety $A$, we have a short exact sequence

$$
0 \rightarrow A \rightarrow \operatorname{Pic}(A) \rightarrow \mathrm{NS}(A) \rightarrow 0
$$

where the Néron-Severi group $\mathrm{NS}(A)$ is naturally isomorphic to the group of endomorphisms of $A$ which are symmetric under the Rosati involution. If $\operatorname{End}(E)=\mathbb{Z}$ (which holds generically), then this becomes

$$
0 \rightarrow E^{n} \rightarrow \operatorname{Pic}\left(E^{n}\right) \rightarrow \mathrm{NS}\left(E^{n}\right) \rightarrow 0
$$

where $\operatorname{NS}\left(E^{n}\right)$ is the group of symmetric $n \times n$ integer matrices. Moreover, it turns out (as we will discuss in more detail below) that this short exact sequence splits, giving us canonical labels for line bundles up to isomorphism.

This last caveat is quite significant, however; if $\mathcal{L}_{1}$ and $\mathcal{L}_{2}$ represent given classes in $\operatorname{Pic}\left(E^{n}\right)$ and $\mathcal{L}_{3}$ represents their sum, then there exists an isomorphism $\mathcal{L}_{1} \otimes \mathcal{L}_{2} \cong \mathcal{L}_{3}$, but this is only determined up to an overall scalar multiple. Another consequence is that if we have (as we will below) a group $G$ acting on $E^{n}$, a $G$-invariant class in $\operatorname{Pic}\left(E^{n}\right)$ need not specify an equivariant line bundle.

If $E$ is an analytic curve $\mathbb{C} /\langle 1, \tau\rangle$, there is a standard way to avoid these difficulties, namely the theory of theta functions. Indeed, given a cocycle $z \in Z^{1}\left(\pi_{1}\left(E^{n}\right) ; \mathbb{A}\left(\mathbb{C}^{n}\right)^{*}\right)$, we can construct a corresponding line bundle $\mathcal{L}_{z}$, and these bundles satisfy $\mathcal{L}_{z_{1}} \otimes \mathcal{L}_{z_{2}} \cong \mathcal{L}_{z_{1} z_{2}}$ and $g^{*} \mathcal{L}_{z} \cong \mathcal{L}_{g^{*} z}$. Moreover, since $H^{1}\left(\mathbb{Z}, \mathbb{A}(\mathbb{C})^{*}\right)=0$, we can arrange for our cocycles to have trivial restriction along $\mathbb{Z}^{n} \subset\langle 1, \tau\rangle^{n} \cong \pi_{1}\left(E^{n}\right)$. We thus obtain the following description of line bundles on $E^{n}$ : given a symmetric integer matrix $Q$ and constants $C_{1}, \ldots, C_{n} \in \mathbb{C}^{*}$, we could consider the line bundle on $(\mathbb{C} /\langle 1, \tau\rangle)^{n}$ with local sections given by functions on $\mathbb{C}$ satisfying

$$
\begin{aligned}
& f\left(z_{1}, \ldots, z_{i-1}, z_{i}+1, z_{i+1}, \ldots, z_{n}\right)=f\left(z_{1}, \ldots, z_{n}\right), \\
& f\left(z_{1}, \ldots, z_{i-1}, z_{i}+\tau, z_{i+1}, \ldots, z_{n}\right)=C_{i} e\left(-\sum_{j} Q_{i j} z_{j}\right) f\left(z_{1}, \ldots, z_{n}\right),
\end{aligned}
$$

where $e(x):=\exp (2 \pi \sqrt{-1} x)$. These line bundles behave well under tensor product, but in slightly odd ways under pullback; it turns out that we should not quite trivialize the cocycle along $\mathbb{Z}^{n}$ in general, but instead merely insist that it restrict to an appropriate morphism 
$\mathbb{Z}^{n} \rightarrow\{ \pm 1\}$. This leads us to define the line bundle $\mathcal{L}_{Q ; \vec{C}}$ on $(\mathbb{C} /\langle 1, \tau\rangle)^{n}$ as the sheaf with local sections consisting of holomorphic functions satisfying

$$
f(\vec{z}+\vec{x} \tau+\vec{y})=\prod_{1 \leq i \leq n}(-1)^{Q_{i i}\left(x_{i}+y_{i}\right)} C_{i}^{x_{i}} \prod_{1 \leq i, j \leq n} e\left(-Q_{i j} x_{i}\left(z_{j}+x_{j} \tau / 2\right)\right) f(\vec{z}),
$$

for $\vec{x}, \vec{y} \in \mathbb{Z}^{n}$. Note that this does not quite solve the problem as stated, since it gives multiple representatives for each line bundle (multiplying $f$ by the nowhere vanishing entire function $e\left(z_{i}\right)$ multiplies $C_{i}$ by $e(\tau)$ ), but still makes it straightforward to control equivariant structures on line bundles, as well as some of the more gerbe-like structures we need to consider below.

Although this suffices for many purposes, we would like to have an algebraic solution to this problem. Not only is our construction below essentially algebraic in nature, there are also some indications that it may prove useful in later work to be able to consider versions defined over finite fields. (In particular, see the use in [1] of finite field instances of the Sklyanin algebra in proving the latter is Noetherian, of particular interest given that we do not yet have a proof that our algebras are Noetherian; see also unpublished work of Bezrukavnikov and Okounkov.) A first step towards this is to observe that we are not really interested in constructing things over a particular curve; rather, we wish to have constructions that apply to all curves. In other words, what we truly want to understand are line bundles on the $n$th fiber power $\mathcal{E}^{n}$ of the universal curve $\mathcal{E}$ over the moduli stack $\mathcal{M}_{1,1}$ of elliptic curves.

Remark. For those readers who may be unfamiliar with stacks, it is worth noting that there is an equivalence between statements about line bundles on $\mathcal{E}^{n}$ (or $\mathcal{M}_{1,1}$ ) and statements about equivariant line bundles on an appropriate scheme-with-group-action. This comes from the fact that each of these stacks is a quotient stack. Indeed, a choice of a nonzero holomorphic differential $\omega$ on an elliptic curve on which 6 is invertible ${ }^{2}$ determines a unique expression of the curve as a Weierstrass curve: the compactification of a curve $y^{2}=x^{3}+a_{4} x+a_{6}$, with differential $\mathrm{d} x / 2 y$. It follows immediately that for any family of elliptic curves $E / S$ with 6 invertible on $S$, there is a $\mathbb{G}_{m}$-torsor $T$ over $S$ such that $E_{T}$ is given by such an equation: simply take $T$ to be the torsor of nonzero holomorphic differentials! It follows that there is a natural isomorphism between the (symmetric monoidal) category of line bundles on $\mathcal{M}_{1,1}[1 / 6]$ (or $\mathcal{E}^{n}[1 / 6]$ ) and the category of $\mathbb{G}_{m}$-equivariant line bundles on $\operatorname{Spec}\left(\mathbb{Z}\left[1 / 6, a_{4}, a_{6}, 1 / \Delta\right]\right.$ ) (or the corresponding family of abelian varieties). Indeed, if we are given a $\mathbb{G}_{m}$-equivariant line bundle on the family of Weierstrass curves (or, for $\mathcal{E}^{n}$, on the appropriate family of abelian varieties), then for any family of elliptic curves over $S$ (resp. with $n$ additional sections), we obtain an induced $\mathbb{G}_{m}$-equivariant bundle on the $\mathbb{G}_{m}$-torsor $T$, and this descends naturally to a line bundle on $S$ (satisfying the requisite compatibility conditions). Conversely, any line bundle on the stack induces a line bundle on the corresponding family of Weierstrass curves, and the compatibility conditions make this line bundle naturally equivariant. This equivalence would allow one to entirely eliminate stacks from the discussion below, at the cost of requiring some additional bookkeeping in the arguments to keep track of equivariance.

The theory of Jacobi forms $[8,13,14,17]$ gives us an approach to this at the analytic level. Analytically, $\mathcal{E}^{n}$ is the quotient of $\mathbb{C}^{n} \times \mathbb{H}$ by the appropriate action of $\mathbb{Z}^{2 n} \rtimes \mathrm{SL}_{2}(\mathbb{Z})$, and thus again we may specify line bundles via cocycles. This leads to the following definition: Given a symmetric integer matrix $Q$ (the "level") and an integer $w$ (the "weight"), we define the line bundle $\mathcal{L}_{Q, w}$ on the complex locus of $\mathcal{E}^{n}$ to be the sheaf with local sections consisting of functions $f\left(z_{1}, \ldots, z_{n} ; \tau\right)$ such that

$$
\begin{aligned}
& f(\vec{z}+\vec{x} \tau+\vec{y} ; \tau)=(-1)^{\vec{x}^{t} Q \vec{x}+\vec{y}^{t} Q \vec{y}} e\left(-\vec{x}^{t} Q(\vec{z}+\vec{x} \tau / 2)\right) f(\vec{z} ; \tau), \\
& f(\vec{z} /(c \tau+d) ;(a \tau+b) /(c \tau+d))=(c \tau+d)^{w} e\left(c \vec{z}^{t} Q \vec{z} / 2(c \tau+d)\right) f(\vec{z} ; \tau),
\end{aligned}
$$

\footnotetext{
${ }^{2}$ Something similar holds over $\mathbb{Z}$, but with $\mathbb{G}_{m}$ replaced by the group $(y, x) \mapsto\left(a^{3} y+b x+c, a^{2} x+d\right)$.
} 
with $\vec{x}, \vec{y} \in \mathbb{Z}^{n}$ and $\left(\begin{array}{ll}a & b \\ c & d\end{array}\right) \in \mathrm{SL}_{2}(\mathbb{Z})$. (This differs from the usual notion of Jacobi form by virtue of our not imposing any condition at the cusp; we have also allowed $Q$ to have odd diagonal.)

Lemma 2.1. The line bundle $\mathcal{L}_{1,-1}(-[0])$ on the complex locus of $\mathcal{E}$ is trivial, where the divisor $[0]$ is the image of the identity section $0: \mathcal{M}_{1,1} \rightarrow \mathcal{E}$.

Proof. We first observe that the function

$$
\begin{aligned}
\vartheta(z ; \tau) & :=\frac{(e(z / 2)-e(-z / 2)) \prod_{1 \leq j}(1-e(j \tau+z))(1-e(j \tau-z))}{\prod_{1 \leq j}(1-e(j \tau))^{2}} \\
& =\frac{\sum_{k \in 1 / 2+\mathbb{Z}}(-1)^{k-1 / 2} e\left(k z+k^{2} \tau / 2\right)}{\sum_{k \in 1 / 2+\mathbb{Z}}(-1)^{k-1 / 2} k e\left(k^{2} \tau / 2\right)}
\end{aligned}
$$

is a global section of $\mathcal{L}_{1,-1}$. This follows immediately from the standard transformation law for Jacobi theta functions together with the transformation law for the Dedekind eta function $e(\tau / 24) \prod_{1 \leq j}(1-e(j \tau))$. (Note that in each case, the overall transformation law involves complicated arithmetic characters, but these turn out to cancel.) This is holomorphic on $\mathbb{C} \times \mathbb{H}$, and for each $\tau \in \mathbb{H}$ is a nonzero function vanishing only on the lattice $\langle 1, \tau\rangle$; thus the corresponding section of $\mathcal{L}_{1,-1}$ has divisor [0], establishing triviality as required.

Remark. The function $\vartheta(z ; \tau)$ may be expressed in the standard multiplicative notation for theta functions in elliptic special function theory as $\vartheta(z ; \tau)=\frac{-x^{-1 / 2} \theta_{p}(x)}{(p ; p)_{\infty}^{2}}$, where $x=e(z)$, $p=e(\tau)$.

It follows in particular that we can extend $\mathcal{L}_{1,-1}$ (or, rather, an algebraic representative of its isomorphism class!) to the entirety of $\mathcal{E}$ : simply take the line bundle $\mathcal{O}_{\mathcal{E}}([0])$. Pulling this back through a homomorphism $g: \mathcal{E}^{n} \rightarrow \mathcal{E},\left(x_{1}, \ldots, x_{n}\right) \mapsto \sum_{i} g_{i} x_{i}$, gives an algebraic version of $\mathcal{L}_{g^{t} g,-1}$, and thus by taking tensor products an algebraic version of $\mathcal{L}_{Q, w}$ in general. Of course, there is a potential issue here of uniqueness. Up to isomorphism, this is settled by the following.

Proposition 2.2. The Picard group of $\mathcal{E}^{n}$ is a canonically split extension of $\operatorname{Pic}\left(\mathcal{M}_{1,1}\right) \cong \mathbb{Z} / 12 \mathbb{Z}$ by the Néron-Severi group of the generic fiber.

Proof. That $\operatorname{Pic}\left(\mathcal{M}_{1,1}\right) \cong \mathbb{Z} / 12 \mathbb{Z}$ is standard (see [10] for an extension to fairly general base changes), as is the fact that we can represent the restrictions of such line bundles to the complex locus via sheaves of modular forms of given weight. (The reduction mod 12 then comes from the fact that the discriminant $\Delta(\tau)$ is a holomorphic form of weight 12 , nowhere vanishing on the smooth locus.) Moreover, the identity section $\mathcal{M}_{1,1} \rightarrow \mathcal{E}^{n}$ gives rise to a splitting of the natural pullback morphism $\operatorname{Pic}\left(\mathcal{M}_{1,1}\right) \rightarrow \operatorname{Pic}\left(\mathcal{E}^{n}\right)$. The above construction moreover shows that the natural map from $\operatorname{Pic}\left(\mathcal{E}^{n}\right)$ to the Néron-Severi group of the generic fiber is surjective. (Note that since $\mathcal{M}_{1,1}$ is a stack, "the generic fiber" does not quite make sense, but it suffices to base change to some smooth curve covering $\mathcal{M}_{1,1}$, say by imposing a full level 3 or full level 4 structure.)

It thus remains only to show that if $\mathcal{L}$ is a line bundle on $\mathcal{E}^{n}$ which on the generic fiber is algebraically equivalent to the trivial divisor, then every fiber of $\mathcal{L}$ is trivial, and thus $\mathcal{L}$ is the pullback of a line bundle on $\mathcal{M}_{1,1}$. Since an algebraically trivial line bundle on an abelian variety gives rise to a point of the dual variety, and $\mathcal{E}^{n}$ is principally polarized via the product polarization, we find that $\mathcal{L}$ induces a section $\mathcal{M}_{1,1} \rightarrow \mathcal{E}^{n}$, and we need merely show that this is the identity section. The elliptic curve $y^{2}+t x y=x^{3}+t^{5}$ over $\mathbb{C}(t)$ has trivial Mordell-Weil group, and thus the pullback of any section $\mathcal{M}_{1,1} \rightarrow \mathcal{E}^{n}$ to this elliptic curve is trivial. Since the corresponding map $\operatorname{Spec}(\mathbb{C}(t)) \rightarrow \mathcal{M}_{1,1}$ is dominant, it follows that any section $\mathcal{M}_{1,1} \rightarrow \mathcal{E}^{n}$ is generically trivial, and thus everywhere trivial since this is a closed condition. 
Thus each line bundle on $\mathcal{E}^{n}$ is determined by its weight (the restriction to the zero section as an element of $\left.\operatorname{Pic}\left(\mathcal{M}_{1,1}\right) \cong \mathbb{Z} / 12 \mathbb{Z}\right)$ and polarization (the class in the Néron-Severi group of the generic fiber). It will be convenient going forward to represent the polarization as a symmetric integer matrix $Q$ or as the corresponding quadratic polynomial $\vec{z} Q \vec{z}^{t} / 2$; the latter will be particularly convenient when we have assigned names to the coordinates in $\mathcal{E}^{n}$.

Let us thus choose for each symmetric integer matrix $Q$ and integer $w \in \mathbb{Z}$ a line bundle $\mathcal{L}_{Q, w}$ (unique up to isomorphism) which restricts to the bundle of weight $w$ on the identity section and induces the symmetric endomorphism $Q$ on the generic fiber of $\mathcal{E}^{n}$. This very nearly solves our problem of constructing line bundles with consistent isomorphisms under tensor product and pullbacks, by virtue of the fact that there are very few global units on $\mathcal{E}^{n}$. Indeed, since $\mathcal{E}^{n}$ is proper over $\mathcal{M}_{1,1}$, the global units on $\mathcal{E}^{n}$ are just the global units on $\mathcal{M}_{1,1}$, and these are easily seen to consist precisely of elements of the form $\pm \Delta^{l}$ for $l \in \mathbb{Z}$. But in fact the same thing that gives us a splitting of the Picard group gives us a natural way to rigidify things completely.

Definition 2.3. For an integer $w$, a weight $w$ trivialization of a line bundle $\mathcal{L}$ on $\mathcal{E}^{n}$ is an isomorphism

$$
0^{*} \mathcal{L} \cong\left(0^{*} \omega_{\mathcal{E} / \mathcal{M}_{1,1}}\right)^{w}
$$

We now enhance our data as follows: $\mathcal{L}_{Q, w}$ is not just a line bundle in the appropriate isomorphism class; rather, it is such a line bundle together with a choice of weight $w$ trivialization. We also insist that $\mathcal{L}_{0,0}=\mathcal{O}_{\mathcal{E}^{n}}$ with the obvious trivialization. (Here we now take $w$ an integer rather than a class mod 12 , as trivializing $0^{*} \omega_{\mathcal{E} / \mathcal{M}_{1,1}^{12}}$ requires choosing one of $\Delta$ or $-\Delta$. This is not a significant issue, however, and in fact should allow us to extend everything to the cusps of $\mathcal{M}_{1,1}$.)

Theorem 2.4. There is a family of natural isomorphisms

$$
\mathcal{L}_{Q_{1}, w_{1}} \otimes \mathcal{L}_{Q_{2}, w_{2}} \cong \mathcal{L}_{Q_{1}+Q_{2}, w_{1}+w_{2}}
$$

agreeing with the obvious isomorphisms when $\left(Q_{1}, w_{1}\right)=(0,0)$ or $\left(Q_{2}, w_{2}\right)=(0,0)$, and, for any linear transformation $g: \mathbb{Z}^{n} \rightarrow \mathbb{Z}^{m}$ (with induced homomorphism $g: \mathcal{E}^{n} \rightarrow \mathcal{E}^{m}$ ), a family of natural isomorphisms

$$
g^{*} \mathcal{L}_{Q, w} \cong \mathcal{L}_{g^{t} Q g, w},
$$

agreeing with the obvious isomorphism when $g=1$. Moreover, these are compatible in the sense that any isomorphism

$$
\bigotimes_{1 \leq i \leq n} g_{i}^{*} \mathcal{L}_{Q_{i}, w_{i}} \rightarrow \mathcal{L}_{\sum_{i} g_{i}^{t} Q_{i} g_{i}, \sum_{i} w_{i}}
$$

constructed from these ingredients is invariant under permutations of the tensor factors and independent of the order in which the above isomorphisms are applied.

Proof. In either case, not only are the bundles isomorphic, but so are their pullbacks through 0 (e.g., since $0^{*} g^{*}=0^{*}$ ), and the respective trivializations actually induce a specific choice of isomorphism of the pullbacks. Since both isomorphisms are determined up to a global unit on $\mathcal{M}_{1,1}$, rigidifying the pullback suffices to rigidify the desired isomorphisms. Similarly, the compatibility conditions certainly hold up to a scalar factor, and pulling back through $0^{*}$ shows that that scalar must be 1 .

To specify (meromorphic) sections of such line bundles, we would like to have an analogue of the Jacobi theta function. Here we have the following. 
Lemma 2.5. The line bundle $\mathcal{O}_{\mathcal{E}}([0])$ on $\mathcal{E}$ has a natural weight -1 trivialization.

Proof. We need an isomorphism

$$
0^{*} \mathcal{O}_{\mathcal{E}}([0]) \otimes 0^{*} \omega_{\mathcal{E} / \mathcal{M}_{1,1}} \cong \mathcal{O}_{\mathcal{M}_{1,1}}
$$

or equivalently

$$
0^{*}\left(\omega_{\mathcal{E} / \mathcal{M}_{1,1}}([0])\right) \cong \mathcal{O}_{\mathcal{M}_{1,1}}
$$

but this is just adjunction.

Remark. Note that the isomorphism coming from adjunction simply takes a differential with simple pole at 0 to its residue. In particular, the natural isomorphism $[-1]^{*} \mathcal{O}_{\mathcal{E}}([0]) \cong \mathcal{O}_{\mathcal{E}}([0])$ negates the trivialization.

Definition 2.6. Let $\mathcal{L}_{1,-1}$ be the chosen line bundle with trivialization on $\mathcal{E}$. Then the global section $\vartheta \in \Gamma\left(\mathcal{L}_{1,-1}\right)$ is the image of 1 under the isomorphism $\mathcal{O}_{\mathcal{E}}([0]) \cong \mathcal{L}_{1,-1}$ respecting the trivialization.

Note that the function $\vartheta$ considered above was normalized so that

$$
\operatorname{Res}_{z=0} \frac{\mathrm{d} z}{\vartheta(z ; \tau)}=1
$$

and thus the two definitions essentially agree on the analytic locus. (This is not quite correct, since the analytic definition of $\mathcal{L}_{1,-1}$ only gives something isomorphic to an algebraic bundle, but the residue condition implies that there is a system of isomorphisms between the algebraic versions of $\mathcal{L}_{Q, w}$ and their analytic versions satisfying appropriate compatibility conditions along tensor products and pullbacks and taking $\vartheta$ to $\vartheta(; \tau)$.) This gives us the following consequence, a very powerful way of constructing functions on powers $\mathcal{E}^{n}$.

Theorem 2.7. Let $c_{i j}, 1 \leq i \leq l, 1 \leq j \leq n$ and $m_{i}, 1 \leq i \leq l$ be integers such that $\sum_{1 \leq i \leq l} m_{i}=0$ and $\sum_{1 \leq i \leq l} m_{i} c_{i j} c_{i k}=0$ for $1 \leq j, k \leq n$. Then there is a (unique) meromorphic function on $\mathcal{E}^{n}$, defined on every fiber, which on the complex locus restricts to $\prod_{1 \leq i \leq l} \vartheta\left(\sum_{j} c_{i j} z_{j} ; \tau\right)^{m_{i}}$.

Proof. We may view each $c_{i}$ as a morphism $\mathcal{E}^{n} \rightarrow \mathcal{E}$, and find that $\prod_{1 \leq i \leq l}\left(c_{i}^{*} \vartheta\right)^{m_{i}}$ is a meromorphic section of the line bundle

$$
\bigotimes_{1 \leq i \leq l}\left(c_{i}^{*} \mathcal{L}_{1,-1}\right)^{m_{i}} \cong \mathcal{L}_{\sum_{i} m_{i} c_{i}^{t} c_{i},-\sum_{i} m_{i}} \cong \mathcal{O}_{\mathcal{E}^{n}}
$$

with both maps canonical. In other words, the given product of sections of line bundles determines a meromorphic function on $\mathcal{E}^{n}$, the divisor of which manifestly has no vertical components.

Remark 2.8. By a very mild abuse of notation, we will write the functions constructed in this way as $\prod_{1 \leq i \leq l} \vartheta\left(\sum_{j} c_{i j} z_{j}\right)^{m_{i}}$, and similarly for the analogous meromorphic sections of line bundles $\mathcal{L}_{Q, w}$.

Remark 2.9. Note that since $[-1]^{*}$ negates the natural trivialization of $\mathcal{O}_{\mathcal{E}}([0])$, we have the identity $\vartheta(-z)=-\vartheta(z)$. 
Remark 2.10. Of course, if we multiply by a suitable power of $\Delta$, we can construct similar functions under the weaker assumption that $\sum_{i} m_{i}$ is a multiple of 12 . On the other hand, with the constraints as given, there is a natural limit at the cusp of $\mathcal{M}_{1,1}$, namely the rational function

$$
\prod_{j} x_{j}^{-\sum_{i} m_{i} c_{i j} / 2} \prod_{1 \leq i \leq l}\left(1-\prod_{j} x_{j}^{c_{i j}}\right)^{m_{i}}
$$

on $\left(\mathbb{C}^{*}\right)^{n}=e(\mathbb{C})^{n}$ (or more generally $\mathbb{G}_{m}^{n}$ ). Note that $\sum_{i} m_{i} c_{i j}$ is even since $\sum_{i} m_{i} c_{i j}^{2}=0$, so this is indeed well-defined. This gives a useful method for sanity-checking calculations, by verifying that the result is correct in this limit. One can also take a limit as all of the variables approach 0; this is ill-defined, but blowing up the 0 section gives the function

$$
\prod_{1 \leq i \leq l}\left(\sum_{j} c_{i j} y_{j}\right)^{m_{i}}
$$

on the exceptional $\mathbb{P}^{n-1}$ (as long as we avoid the finitely many characteristics in which one of the factors is identically 0 ).

It is worth noting that there is an alternate approach to the theorem which, while it does not help us deal with line bundles, is more powerful in one important respect: it gives a reasonable algorithm for evaluating such functions at specific points (i.e., on $n$-tuples of points of specific elliptic curves, say over a finite field). We choose (to make the appropriate induction work) a differential $\omega$ on $E$, allowing us to eliminate the constraint $\sum_{i} m_{i}=0$. The corresponding trivialization of the line bundle $\mathcal{O}_{\mathcal{E}}(-d[0])$ can then be computed as follows: choose any uniformizer $u$ at 0 such that $\omega / u$ has residue 1 , and then for any function $f$ with multiplicity $d$ at 0 , express $f=c(f, \omega) u^{d}+O\left(u^{d+1}\right)$ to obtain a "leading coefficient" $c(f, \omega)$. It is easy to see that this leading coefficient is independent of the choice of $u$, and that $c(f, \omega)$ scales in the appropriate way with $\omega$.

Now, we can characterize the function $\prod_{1 \leq i \leq l} \vartheta\left(\sum_{j} c_{i j} z_{j}\right)^{m_{i}}$ up to a scalar multiple by viewing it as a function of $z_{n}$ with specified divisor. If there are no factors depending only on $z_{n}$, then the value of the function along $z_{n}=0$ is then a function of the same form, which can be computed by induction, giving us the requisite scale factor and letting us plug in the specific value of $z_{n}$ desired. If the only factors depending only on $z_{n}$ are powers of $\vartheta\left(z_{n}\right)$, then we use the leading coefficient instead and proceed as before. (The correct leading coefficient is computed by removing powers of $\vartheta\left(z_{n}\right)$ and setting $z_{n}=0$.) Note that in general we can compute the leading coefficient as long as every factor $\vartheta\left(k z_{n}\right)^{m}$ that arises has $k$ invertible (since $\vartheta(k z)$ has leading term $k u$ in characteristic 0 ). Thus if we can construct functions of the form $\frac{\vartheta(k z)}{\vartheta(z)^{k^{2}}}$ directly, we could eliminate those factors before computing the leading coefficient. Since $\vartheta(k z)=-\vartheta(-k z)$, we reduce to the case $k>1$. Divisor considerations then tell us that such a function must be proportional to the $k$-division polynomial (as defined in [34, Example 3.7]), and we find in fact that the $k$-division polynomial has the correct leading term in characteristic 0 , so gives the desired function on general $E$.

Note that although we are writing $\vartheta(z)$ as a function, the fact that it is only a section of a line bundle means that we must be somewhat cautious when specializing. In particular, consider the ratio $\vartheta(u+z) / \vartheta(u)$, a section of the line bundle with polarization $z^{2} / 2+u z$ and weight 0 . The restriction of this line bundle to the hypersurface $z=0$ is trivial (and in a natural way given our global choices of trivializations), and thus $\vartheta(u+z) / \vartheta(u)$ restricts to a function on this hypersurface, which we can verify is equal to 1 . There is, however, an issue in the analytic setting, as the algebraic hypersurface $z=0$ corresponds to the analytic hypersurfaces $z=x+y \tau$, 
$x, y \in \mathbb{Z}$, and $\vartheta(u+x+y \tau ; \tau) / \vartheta(u ; \tau)$ is of course nontrivial for most values of $x, y$. (For $z=0$, there is still no issue, naturally.)

In contrast, if we restrict the ratio $\vartheta(u+m z) / \vartheta(u)$ to the hypersurface $m z=0$, then there are difficulties even algebraically. The difficulty here is that although the restriction of the line bundle is trivial on every fiber over $\mathcal{E}[\mathrm{m}]$, it is not canonically trivial, and thus the restriction could be a nontrivial line bundle on $\mathcal{E}[m]$, and even if trivial, the given section need not restrict to 1 .

Indeed, let $\mathfrak{q} \in \mu_{2}(E[2])$ be the character of the natural [-1]-equivariant structure on $\mathcal{L}_{1,-1}$ (i.e., such that the action on the fiber at 0 is trivial) on the fixed subscheme $E[2]$. Note that $\mathfrak{q}(0)=1$ and when the characteristic is not 2 is -1 at the remaining 2-torsion points; in general, it is a $\mu_{2}$-valued quadratic form such that the induced bilinear form is the Weil pairing.

Proposition 2.11. The restriction of the function

$$
\frac{\vartheta(u-z) \vartheta(v-z) \vartheta(u+v+z)}{\vartheta(u+z) \vartheta(v+z) \vartheta(u+v-z)}
$$

to the hypersurface $2 z=0$ is given by $\mathfrak{q}(z)$.

Proof. To compute $\mathfrak{q}$, we need merely express $\mathcal{L}_{1,-1}$ as $\mathcal{O}(D)$ for a suitable divisor $D$ disjoint from the 2-torsion, at which point $\mathfrak{q}$ is the restriction to $E[2]$ of the unique function $f$ with divisor $D-[-1]^{*} D$ such that $f(0)=1$. Taking $D=[u]+[v]-[u+v]$ gives the desired result.

We note the following fact useful for simplifying products of such factors.

Proposition 2.12. For any integer $m \geq 1$, the restriction of the function

$$
\frac{\vartheta(u+m z) \vartheta(u+w) \vartheta(v) \vartheta(v+w+m z)}{\vartheta(u) \vartheta(u+w+m z) \vartheta(v+m z) \vartheta(v+w)}
$$

to the hypersurface $m z=0$ is 1 .

Proof. Indeed, the original function is a rational function on $\mathcal{E}^{4}$, which may be described as the unique elliptic function of $u$ with the appropriate divisor and taking the value 1 at $u=v$. On the hypersurface $m z=0$, this elliptic function of $u$ has divisor 0 , and is thus the function 1 .

Remark. It follows that the restriction to $m z=0$ of

$$
\frac{\vartheta(u) \vartheta(u+v+m z)}{\vartheta(u+m z) \vartheta(u+v)}
$$

is independent of $u$ and in a suitable sense a homomorphism in $v$. Of course, again, it is only a section of a line bundle which is isomorphic to the pullback of a line bundle on $\mathcal{E}[\mathrm{m}]$, but not canonically so. In the analytic setting, the corresponding ratio as a function of $v$ indeed gives a homomorphism from the universal cover; the specific homomorphism depends on the choice of representative of the given $m$-torsion point, and for some $m$-torsion points cannot be made trivial.

Note that since both lemmas are stated in terms of actual functions on the moduli stack, we may replace the respective variables by arbitrary homomorphisms $\mathcal{E}^{n} \rightarrow \mathcal{E}$.

In addition to line bundles and their sections, we will also need some gerbe-ish structures. The objects we want to consider are "equivariant gerbes" (more precisely, equivariant structures on the trivial gerbe; all equivariant gerbes considered in the present work will have trivial 
underlying gerbe): a system of line bundles $\mathcal{Z}_{g}$ associated to $g \in G \subset \mathrm{GL}_{n}(\mathbb{Z})$ along with morphisms $\zeta_{g, h}: \mathcal{Z}_{g} \otimes\left(g^{-1}\right)^{*} \mathcal{Z}_{h} \cong \mathcal{Z}_{g h}$ making all diagrams

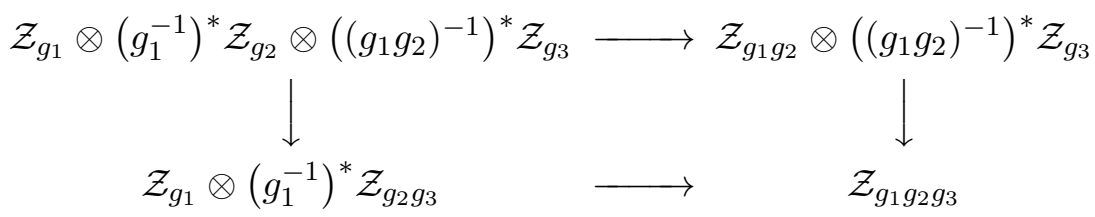

commute. This, of course, is easy to construct: given any 1-cocycle for $G$ valued in pairs $(Q, w)$, we may take $\mathcal{Z}_{g}$ to be $\mathcal{L}_{Q_{g}, w_{g}}$ and $\zeta_{g, h}$ to be the natural isomorphism

$$
\zeta_{g, h}: \mathcal{L}_{Q_{g}, w_{g}} \otimes\left(g^{-1}\right)^{*} \mathcal{L}_{Q_{h}, w_{h}} \cong \mathcal{L}_{Q_{g}+g^{-t} Q_{h} g^{-1}, w_{g}+w_{h}}=\mathcal{L}_{Q_{g h}, w_{g h}} .
$$

The situation becomes more complicated if we want to also construct meromorphic sections of such gerbes: i.e., a system of meromorphic sections $z_{g} \in \mathcal{Z}_{g}$ such that $\zeta_{g, h}\left(z_{g} \otimes\left(g^{-1}\right)^{*} z_{h}\right)=z_{g h}$. In this case, we do not have a completely general solution, but there is an important special case.

Consider first the case $G=\mathbb{Z}$, with generator acting on $\mathcal{E}^{2}$ by $(z, q) \mapsto(z+q, q)$. There is a natural equivariant gerbe with meromorphic section such that $\mathcal{Z}_{1}=\mathcal{L}_{z^{2} / 2,-1}$ and $z_{1}=\vartheta(z)$. Indeed, we then find more generally that $\mathcal{Z}_{k}$ is the natural line bundle of weight $-k$ with polarization

$$
(z, q) Q(z, q)^{t} / 2=k \frac{z^{2}}{2}+\frac{k(k-1)}{2} q z+\frac{k(2 k-1)(k-1)}{6} \frac{q^{2}}{2},
$$

and

$$
z_{k}=\vartheta(z ; q)_{k}:= \begin{cases}\prod_{0 \leq i<k} \vartheta(i q+z), & k \geq 0 \\ \prod_{1 \leq i \leq-k} \vartheta(-i q+z)^{-1}, & k \leq 0\end{cases}
$$

We will denote this meromorphic section of an equivariant gerbe by $\Gamma_{q}(z)$, which we refer to as an "elliptic Gamma function". Of course, this is even less a function than $\vartheta$, but in the analytic setting one can indeed replace $\Gamma_{q}(z)$ by a suitable meromorphic solution of the functional equation $\Gamma_{q}(q+z)=\vartheta(z) \Gamma_{q}(z)$. Of course, this only determines $\Gamma_{q}$ up to multiplication by invertible $q$-periodic functions, so the resulting meromorphic function is far from unique. One such solution (for $q$ in the upper half-plane) is

$$
\Gamma_{q}(z ; \tau):=\left(-\prod_{1 \leq j}(1-e(j \tau))^{2}\right)^{-z / q} e(-z(z-q) / 4 q) \prod_{0 \leq j, k} \frac{1-e((j+1) \tau+(k+1) q-z)}{1-e(j \tau+k q+z)}
$$

this depends on a choice of $\log \left(-\prod_{1 \leq j}(1-e(j \tau))^{2}\right)$, but this will not matter for our purposes. Here the double product is just the usual elliptic Gamma function [30].

More generally, for given morphisms $q, z: \mathcal{E}^{n} \rightarrow \mathcal{E}$, we may pull this formal symbol back to $\mathcal{E}^{n}$. This is tricky to deal with in complete generality, but if we fix $q$ and vary $z$, we may consider general products

$$
\prod_{1 \leq i \leq l} \Gamma_{q}\left(\vec{\alpha}_{i} \cdot \vec{z}\right)^{m_{i}}
$$

If the corresponding element $\sum_{i} m_{i}\left[\vec{\alpha}_{i}\right]$ of $\mathbb{Z}\left[\operatorname{Hom}\left(\mathcal{E}^{n}, \mathcal{E}\right) / q\right]$ is trivial, then this formal product may be resolved into a product of $\vartheta$ functions using the functional equation. Moreover, since for 
each congruence class the corresponding subproduct may be pulled back from $\mathcal{E}^{2}$, we find that the resulting product of $\vartheta$ functions is independent of any choices that may have been made.

More generally, if $G \subset \mathrm{GL}_{n}(\mathbb{Z})$ fixes $q$ and the element $\sum_{i} m_{i}\left[\vec{\alpha}_{i}\right] \in \mathbb{Z}\left[\operatorname{Hom}\left(\mathcal{E}^{n}, \mathcal{E}\right) / q\right]$, then the ratio of the formal product and its pullback under $g \in G$ will always resolve to a product of $\vartheta$ functions, and thus gives an equivariant-gerbe-with-meromorphic-section which we refer to as the coboundary of the formal symbol. Note in particular that the hypothesis always holds for the (translation) subgroup of $\mathrm{GL}_{n}(\mathbb{Z})$ that acts trivially on both $q$ and the quotient $\operatorname{Hom}\left(\mathcal{E}^{n}, \mathcal{E}\right) / q$; in the cases of interest (affine Weyl groups), the intersection of $G$ with this subgroup will be cofinite in both groups, and it will be relatively straightforward to check that individual instances give rise to sections of gerbes.

We should caution the reader that there is a mild subtlety when it comes to the reflection principle. Although the product $\Gamma_{q}(z) \Gamma_{q}(q-z)$ corresponds to the trivial equivariant gerbe (it has polarization 0 and weight -1 (see below), both of which are invariant under $z \mapsto z+q$ ), the corresponding meromorphic section is not quite trivial: one finds that $\Gamma_{q}(z) \Gamma_{q}(q-z)$ is negated by the translation $z \mapsto z+q$.

On the other hand, the multiplication principle $\Gamma_{q}(z)=\prod_{0 \leq j<k} \Gamma_{k q}(z+j q)$ for integer $k>0$ does work, as both sides truly do correspond to the same gerbe-with-section. This also works for negative $k: \Gamma_{q}(z)=\prod_{1 \leq j \leq-k} \Gamma_{k q}(z-j q)^{-1}$. Using this, one can make sense of products of elliptic Gamma functions with varying $q$ as long as the different $q$ that appear have a common multiple. Note in particular the special case $\Gamma_{q}(z)=\Gamma_{-q}(z-q)^{-1}$.

We will of course want to know the polarizations and weights of the line bundles associated to a given such gerbe section, which reduces to knowing the polarization and weight when such a product resolves to a product of $\vartheta$ functions. There is, in fact, a very simple bookkeeping procedure for determining this. Define the polarization of $\Gamma_{q}(z)$ to be $\frac{z(z-q)(2 z-q)}{12 q}$, and the weight of $\Gamma_{q}(z)$ to be $-\frac{z}{q}$, extended to products of pullbacks in the obvious way. By this definition, the polarization of the formal product $\Gamma_{q}(q+z) / \Gamma_{q}(z)$ is $z^{2} / 2$ and the weight is -1 , agreeing with the polarization and weight of $\vartheta(z)$. It follows more generally that the polarization and weight of a product of powers of elliptic Gamma functions is consistent with the usual notion whenever the product resolves to a product of $\vartheta$ functions. (In particular, if the product resolves and the polarization and weight are trivial, then it resolves to an honest function on $\mathcal{E}^{n}$.) Of course, when considering the associated gerbe-with-section, only the $z$-dependent portion of the polarization matters.

One should note here that not every cocycle valued in pairs $(Q, w)$ comes from a product of $\Gamma_{q}$ symbols; for instance, any product of symbols $\Gamma_{q}(a z+b q)$ with trivial polarization has weight of the form $12 c z / q+d$, so that every line bundle in the coboundary has weight a multiple of 12 . (There are also some additional parity issues for $n>1$.) Of course, it is conceivable that the cocycles violating these congruence conditions do not have any consistent family of meromorphic sections.

Note that if one wishes to convert a product of standard elliptic Gamma functions into a product of symbols $\Gamma_{q}$, one must first use the reflection principle to eliminate appearances of $p$ from the arguments (which, if there is a balancing condition that involves $p$ will require shifting some of the variables by $p$ first). If the result has $\sum_{i} m_{i}\left(\sum_{i} \vec{\alpha}_{i} \cdot z_{i}\right)^{2}=\sum_{i} m_{i}\left(\sum_{i} \vec{\alpha}_{i} \cdot z_{i}\right)=0$, then replacing the standard Gamma functions by the explicit meromorphic solution given above for the functional equation of $\Gamma_{q}$ will have no effect on the resulting meromorphic function.

Since we now have a method for constructing equivariant line bundles on $\mathcal{E}^{n}$, it is natural to ask about the corresponding representations of $G$ on global sections; in particular, we will want to understand the space of $G$-invariant global sections. It turns out that if we want to extend the standard analytic approach to this question to algebraic curves, we will need to extend the above construction to cover certain abelian varieties isogenous to $\mathcal{E}^{n}$.

To be precise, for a positive integer $N$ let $\mathcal{X}_{0}(N)$ denote the moduli stack of elliptic curves $E_{1}$ equipped with a cyclic $N$-isogeny $\phi: E_{1} \rightarrow E_{N}$, with universal curves denoted by $\mathcal{E}=\mathcal{E}_{1}, \mathcal{E}_{N}$. 
(Here "cyclic" is in the sense of [15]; in particular, note that in characteristic $p$, any isogeny of degree $p^{k}$ is cyclic.) For each divisor $d \mid N$, there is a corresponding factorization $\phi=\phi_{N, d} \circ \phi_{d, 1}$, where $\phi_{d, 1}: \mathcal{E}_{1} \rightarrow \mathcal{E}_{d}, \phi_{N, d}: \mathcal{E}_{d} \rightarrow \mathcal{E}_{N}$ are cyclic isogenies of degrees $d$ and $N / d$ respectively. For $d=p$ prime, this is constructed as follows: on the locus where the $p$-part of ker $\phi$ is étale, $\mathcal{E}_{p}$ is the quotient by the $p$-torsion of ker $\phi$; on the complementary locus, where the $p$-part of ker $\phi$ is nonreduced, $\phi_{1, p}$ is the Frobenius isogeny. (Per [15], this rule gives the correct limit to make $\mathcal{E}_{p}$ a flat family.) In either case, $\operatorname{ker} \phi_{1, p} \subset \operatorname{ker} \phi$, and thus $\phi$ factors as required, and we find that $\phi_{p, N}$ is again cyclic. We thus have induced factorizations for every $d \mid N$, and it is easy to see that they are all compatible. More precisely, for any pair $d_{1}\left|d_{2}\right| N$, we obtain an isogeny $\phi_{d_{2}, d_{1}}: \mathcal{E}_{d_{1}} \rightarrow \mathcal{E}_{d_{2}}$, and $\phi_{d_{3}, d_{2}} \circ \phi_{d_{2}, d_{1}} \cong \phi_{d_{3}, d_{1}}$. We also, of course, have similarly compatible isogenies $\phi_{d_{1}, d_{2}}: \mathcal{E}_{d_{2}} \rightarrow \mathcal{E}_{d_{1}}$ obtained by dualizing $\phi_{d_{2}, d_{1}}$.

Lemma 2.13. For any $d_{1}, d_{2} \mid N$, we have $\operatorname{Hom}\left(\mathcal{E}_{d_{1}}, \mathcal{E}_{d_{2}}\right) \cong \mathbb{Z}$, generated by the composition

$$
\phi_{d_{2}, d_{1}}:=\phi_{d_{2}, \operatorname{gcd}\left(d_{1}, d_{2}\right)} \circ \phi_{\operatorname{gcd}\left(d_{1}, d_{2}\right), d_{1}}=\phi_{d_{2}, \operatorname{lcm}\left(d_{1}, d_{2}\right)} \circ \phi_{\operatorname{lcm}\left(d_{1}, d_{2}\right), d_{1}} .
$$

Proof. Let $d_{0}=\operatorname{gcd}\left(d_{1}, d_{2}\right)$ and $d_{3}=\operatorname{lcm}\left(d_{1}, d_{2}\right)$, and let $f: \mathcal{E}_{d_{1}} \rightarrow \mathcal{E}_{d_{2}}$ be any homomorphism. Then $\phi_{d_{0}, d_{2}} \circ f \circ \phi_{d_{1}, d_{0}}$ is an endomorphism of $\mathcal{E}_{d_{0}}$, so must be multiplication by some integer. By degree considerations, that integer must be a multiple of $d_{2} / d_{0}$ and $d_{1} / d_{0}$, and thus (since these are relatively prime) of $d_{1} d_{2} / d_{0}^{2}=d_{3} / d_{0}$. Since $\phi_{d_{0}, d_{2}} \circ \phi_{d_{2}, d_{1}} \circ \phi_{d_{1}, d_{0}}=\left[d_{1} d_{2} / d_{0}^{2}\right]$, the first claim follows. We moreover find that

$$
\phi_{d_{0}, d_{2}} \circ \phi_{d_{2}, d_{3}} \circ \phi_{d_{3}, d_{1}} \circ \phi_{d_{1}, d_{0}}=\phi_{d_{0}, d_{3}} \circ \phi_{d_{3}, d_{0}}=\left[d_{3} / d_{0}\right]
$$

from which the other factorization follows.

For any sequence of divisors $d_{i}$ of $N$, we may consider the corresponding fiber product of curves $\mathcal{E}_{d_{i}}$ over $\mathcal{X}_{0}(N)$; we will generally omit $\mathcal{X}_{0}(N)$ from the product notation. As in the $N=1$ case, morphisms between such products may be expressed as matrices, with the only difference being that the $i j$ entry is now a multiple of the natural isogeny $\phi_{d_{i}, d_{j}}$.

Proposition 2.14. For any $d_{1}, d_{2} \mid N$, there is an isomorphism

$$
\mathcal{E}_{d_{1}} \times \mathcal{E}_{d_{2}} \cong \mathcal{E}_{\operatorname{gcd}\left(d_{1}, d_{2}\right)} \times \mathcal{E}_{\mathrm{lcm}\left(d_{1}, d_{2}\right)} .
$$

Proof. Let $d_{0}=\operatorname{gcd}\left(d_{1}, d_{2}\right), d_{3}=\operatorname{lcm}\left(d_{1}, d_{2}\right)$, and choose $a, b$ so that $a d_{1}-b d_{2}=d_{0}$. We then easily verify that the morphisms

$$
\left(\begin{array}{cc}
a \phi_{d_{0}, d_{1}} & b \phi_{d_{0}, d_{2}} \\
\phi_{d_{3}, d_{1}} & \phi_{d_{3}, d_{2}}
\end{array}\right): \mathcal{E}_{d_{1}} \times \mathcal{E}_{d_{2}} \rightarrow \mathcal{E}_{d_{0}} \times \mathcal{E}_{d_{3}}
$$

and

$$
\left(\begin{array}{cc}
\phi_{d_{1}, d_{0}} & -b\left(d_{2} / d_{0}\right) \phi_{d_{1}, d_{3}} \\
-\phi_{d_{2}, d_{0}} & a\left(d_{1} / d_{0}\right) \phi_{d_{2}, d_{3}}
\end{array}\right): \mathcal{E}_{d_{0}} \times \mathcal{E}_{d_{3}} \rightarrow \mathcal{E}_{d_{1}} \times \mathcal{E}_{d_{2}}
$$

are inverses, giving the desired isomorphism.

It follows immediately that any product of curves $\mathcal{E}_{d}$ is isomorphic to one of the form $\prod_{i} \mathcal{E}_{d_{i}}$, where $1\left|d_{1}\right| \cdots\left|d_{n}\right| N$.

If we attempt to repeat our $N=1$ construction for general $N$, we encounter two difficulties. The first is that for each $d \mid N$, we may obtain a line bundle on $\mathcal{X}_{0}(N)$ by taking the fiber at 1 of the sheaf of differentials on $\mathcal{E}_{d}$, but these line bundles are not quite the same. If $E_{1} \rightarrow E_{d}$ is an étale isogeny, there is no problem: $\phi_{1, d}^{*}$ induces an isomorphism of $\omega_{E_{d}}$ and $\omega_{E_{1}}$, so in particular of their fibers over 1 . However, if $E_{1} \rightarrow E_{d}$ is inseparable, then $\phi_{1, d}^{*}$ actually annihilates $\omega_{E_{d}}$. 
As a result, $\left.\omega_{E_{d}}\right|_{0}$ and $\left.\omega_{E_{1}}\right|_{0}$ differ by a linear combination of components of the fibers of $\mathcal{X}_{0}(N)$ over primes dividing $d$. Of course, if all we want to do is construct line bundles, this is not an issue, but this does mean that the construction of functions in this way will be nontrivial.

The more serious difficulty is that it is no longer the case that $\prod_{i} \mathcal{E}_{d_{i}} \rightarrow \mathcal{X}_{0}(N)$ has only the trivial section. Indeed, we have the following.

Lemma 2.15. Let $N$ be a positive integer, and $d \mid N$. Then the group of sections of $\mathcal{E}_{d}$ over $\mathcal{X}_{0}(N)$ consists entirely of 2-torsion. If both $d$ and $N / d$ are odd, the group is trivial; if precisely one is even, it has rank 1, and if both are even, it has rank 2.

Proof. Since the stack $\mathcal{X}_{0}(N)$ has nontrivial stabilizers, any section of $\mathcal{E}_{d}$ must be invariant under the action of the stabilizer, and is thus preserved by [-1], so is 2-torsion. The structure of the 2-torsion of $\mathcal{E}_{d}$ then follows by considering the image of the corresponding congruence subgroup in $\mathrm{SL}_{2}(\mathbb{Z} / 2 \mathbb{Z})$.

Remark. For more general level structures, it follows from [33, Theorem 5.5] that for any subgroup $\Gamma \subset \mathrm{SL}_{2}(\mathbb{Z} / N \mathbb{Z})$, the group of sections over the corresponding quotient stack $\mathcal{X}(N) / \Gamma$ is $N$-torsion, and isomorphic to the subgroup $\left((\mathbb{Z} / N \mathbb{Z})^{2}\right)^{\Gamma}$.

If $N$ is odd, we may thus conclude as before that the pullback of $\mathcal{O}_{\mathcal{E}_{d_{1}}}([0])$ through $\phi_{d_{1}, d_{2}}$ is indeed fiberwise isomorphic to $\mathcal{O}_{\mathcal{E}_{d_{2}}}([0])^{\operatorname{deg}\left(\phi_{d_{1}, d_{2}}\right)}$. However, if $N$ is even, this is no longer the case; indeed, the pullback of $\mathcal{O}_{E}([0])$ through a 2-isogeny $E^{\prime} \rightarrow E$ is the tensor product of $\mathcal{O}_{E^{\prime}}(2[0])$ by the corresponding 2-torsion line bundle.

It turns out that we can fix this, at the cost of imposing some additional level structure. Let $\mathcal{X}_{0}(2 N, 2)$ be the slight reinterpretation of the stack $\mathcal{X}_{0}(4 N)$ obtained by dividing all of the subscripts of the isogenous curves by 2 ; that is $\mathcal{X}_{0}(2 N, 2)$ classifies cyclic $4 N$-isogenies $\mathcal{E}_{1 / 2} \rightarrow \mathcal{E}_{2 N}$ in terms of the curve $\mathcal{E}_{1}$. Now, it follows from the lemma that for each integer $d \mid N$, the curve $\mathcal{E}_{d}$ has full 2-torsion over $\mathcal{X}_{0}(2 N, 2)$; in addition to the generators of the kernels $\mathcal{E}_{d} \rightarrow \mathcal{E}_{d / 2}$ and $\mathcal{E}_{d} \rightarrow \mathcal{E}_{2 d}$, it also has their sum, which we denote by $\sigma_{d}$. We thus obtain a line bundle $\hat{\mathcal{L}}_{1, \mathcal{E}_{d}}:=\mathcal{O}_{\mathcal{E}_{d}}\left(\left[\sigma_{d}\right]\right) \otimes 0^{*} \mathcal{O}_{\mathcal{E}_{d}}\left(\left[\sigma_{d}\right]\right)^{-1}$ on $\mathcal{E}_{d}$ with trivial fiber over 0 . Note that again $0^{*} \mathcal{O}_{\mathcal{E}_{d}}\left(\left[\sigma_{d}\right]\right)$ is a nontrivial line bundle, though it is actually far better-behaved than $0^{*} \mathcal{O}_{\mathcal{E}_{d}}([0])$. Indeed, $0^{*} \mathcal{O}_{\mathcal{E}_{d}}\left(\left[\sigma_{d}\right]\right)$ is trivial away from the locus where $\sigma_{d}=0$. This can only happen in characteristic 2, and only when the 4-isogeny $\mathcal{E}_{d / 2} \rightarrow \mathcal{E}_{2 d}$ is multiplication by 2 (corresponding to one of the three components of $\mathcal{X}_{0}(2,2)$ in characteristic 2 ).

The merit of using this 2 -torsion point is that it makes everything compatible.

Lemma 2.16. If $d_{1}\left|d_{2}\right| N$ then there are natural isomorphisms

$$
\begin{aligned}
& \phi_{d_{2}, d_{1}}^{*} \hat{\mathcal{L}}_{1, \mathcal{E}_{d_{2}}} \cong \hat{\mathcal{L}}_{1, \mathcal{E}_{d_{1}}^{d_{2} / d_{1}},}, \\
& \phi_{d_{1}, d_{2}}^{*} \hat{\mathcal{L}}_{1, \mathcal{E}_{d_{1}}} \cong \hat{\mathcal{L}}_{1, \mathcal{E}_{d_{2}}}^{d_{2} / d_{1}} .
\end{aligned}
$$

Proof. The two claims are equivalent via the (Atkin-Lehner) involution $\mathcal{X}_{0}(2 N, 2) \cong \mathcal{X}_{0}(2 N, 2)$ that replaces $\mathcal{E}_{1 / 2} \rightarrow \mathcal{E}_{2 N}$ by its dual, so it suffices to prove the first. Since the fibers at the origin of the two bundles are trivial, it suffices to show that the line bundles are isomorphic on each fiber over $\mathcal{X}_{0}(2 N, 2)$, at which point we may take the isomorphism respecting the fibers at the origin. We may also, for convenience, reduce to the case $d_{1}=1, d_{2}=N$.

Being isomorphic is a closed condition, so we may exclude primes dividing $4 N$, and in particular assume that the isogenies are all separable. On a given such curve, the line bundle $\phi_{N, 1}^{*} \hat{\mathcal{L}}_{1, \mathcal{E}_{N}}$ is represented by the divisor

$$
\sum_{x \in \phi_{N, 1}^{-1}\left(\sigma_{N}\right)}[x],
$$


and thus we need to show

$$
\sum_{x \in \phi_{N, 1}^{-1}\left(\sigma_{N}\right)} x=N \sigma_{1},
$$

or equivalently

$$
\sum_{x \in \phi_{N, 1}^{-1}\left(\sigma_{N}-\phi_{N, 1}\left(\sigma_{1}\right)\right)} x=0 .
$$

If $N$ is odd, then $\phi\left(\sigma_{N}\right)=\sigma_{1}$, and this sum becomes

$$
\sum_{x \in \operatorname{ker} \phi_{N, 1}} x=0
$$

as required. Otherwise, we may factor through $\mathcal{E}_{2}$ and thus reduce to the case $N=2$. Then we find that $\phi_{2,1} \sigma_{1}$ is the generator of the kernel of $\phi_{1,2}$, and thus $\sigma_{2}-\phi_{2,1} \sigma_{1}$ is the generator of the kernel of $\phi_{2,4}$. It follows that the preimage of $\sigma_{2}-\phi_{2,1} \sigma_{1}$ consists of the two generators of the kernel of $\phi_{1,4}$, and these add to 0 as required.

Remark. It is worth noting here that there are additional curves in the isogeny class of $\mathcal{E}_{1}$, since after all each of the 2 -torsion points $\sigma_{d}$ itself determines a 2 -isogeny. It is unclear whether we can extend the above family of line bundles to the other curves arising in this way.

Corollary 2.17. For any integer $a,[a]^{*} \hat{\mathcal{L}}_{1, \mathcal{E}_{d}} \cong \hat{\mathcal{L}}_{1, \mathcal{E}_{d}}^{a^{2}}$.

Proof. This is clearly true for $a=0$ and $a=-1$, so we may assume $a>0$. Over $\mathcal{X}_{0}(2 a N, 2)$, we have $[a]=\phi_{d, a d} \circ \phi_{a d, d}$, so that the claim follows immediately from the lemma. Since the isomorphism is natural, it descends to $\mathcal{X}_{0}(2 N, 2)$ as required.

Since $\hat{\mathcal{L}}_{1, \mathcal{E}_{d}}$ represents the standard principal polarization on $\mathcal{E}_{d}$, we also have the following.

Lemma 2.18. For any $d \mid N$, the bundle

$$
\left[x_{1}+x_{2}\right]^{*} \hat{\mathcal{L}}_{1, \mathcal{E}_{d}} \otimes\left[x_{1}\right]^{*} \hat{\mathcal{L}}_{1, \mathcal{E}_{d}}^{-1} \otimes\left[x_{2}\right]^{*} \hat{\mathcal{L}}_{1, \mathcal{E}_{d}}^{-1}
$$

is naturally isomorphic to the Poincaré bundle $\mathcal{P}_{\mathcal{E}_{d}}$ on $\mathcal{E}_{d} \times \mathcal{E}_{d}$.

Theorem 2.19. For any sequence $1\left|d_{1}\right| \cdots\left|d_{n}\right| N$, suppose $\mathcal{L}_{1}$ and $\mathcal{L}_{2}$ are two line bundles on $\prod_{i} \mathcal{E}_{d_{i}}$ obtained as tensor products of pullbacks of bundles $\hat{\mathcal{L}}_{1, \mathcal{E}_{d}}$ through morphisms $\prod_{i} \mathcal{E}_{d_{i}} \rightarrow \mathcal{E}_{d}$. If $\mathcal{L}_{1}$ and $\mathcal{L}_{2}$ represent the same polarization, then they are naturally isomorphic.

Proof. By the theorem of the cube, it suffices to prove this for $n=2$. In this case, it is clear that the images of the bundles $(1 \times 0)^{*} \hat{\mathcal{L}}_{1, \mathcal{E}_{d_{1}}},(0 \times 1)^{*} \hat{\mathcal{L}}_{1, \mathcal{E}_{d_{2}}}$ and $\left(1 \times \phi_{d_{1}, d_{2}}\right)^{*} \mathcal{P}_{\mathcal{E}_{d_{1}}}$ span the group of symmetric endomorphisms of $\mathcal{E}_{d_{1}} \times \mathcal{E}_{d_{2}}$, and thus it will suffice to show that any pullback of $\hat{\mathcal{L}}_{1, \mathcal{E}_{d}}$ is isomorphic to the appropriate product of these bundles (again using the triviality at 0 to fix the isomorphism).

Thus consider a morphism $\psi:=a \phi_{d, d_{1}}+b \phi_{d, d_{2}}: \mathcal{E}_{d_{1}} \times \mathcal{E}_{d_{2}} \rightarrow \mathcal{E}_{d}$. We then have

$$
\psi^{*} \hat{\mathcal{L}}_{1, \mathcal{E}_{d}} \cong\left(a \phi_{d, d_{1}}\right)^{*} \hat{\mathcal{L}}_{1, \mathcal{E}_{d}} \otimes\left(b \phi_{d, d_{2}}\right)^{*} \hat{\mathcal{L}}_{1, \mathcal{E}_{d}} \otimes\left(a \phi_{d, d_{1}} \times b \phi_{d, d_{2}}\right)^{*} \mathcal{P}_{\mathcal{E}_{d}} .
$$

Now,

$$
\left(a \phi_{d, d_{1}}\right)^{*} \hat{\mathcal{L}}_{1, \mathcal{E}_{d}}=[a]^{*} \phi_{\operatorname{gcd}\left(d, d_{1}\right), d_{1}}^{*} \phi_{d, \operatorname{gcd}\left(d, d_{1}\right)}^{*} \hat{\mathcal{L}}_{1, \mathcal{E}_{d}}=\hat{\mathcal{L}}_{1, \mathcal{E}_{d_{1}}}^{a^{2} \operatorname{gcd}\left(d, d_{1}\right)^{2} / d_{1} d},
$$

and similarly for the second term. We also have

$$
\left(a \phi_{d, d_{1}} \times b \phi_{d, d_{2}}\right)^{*} \mathcal{P}_{\mathcal{E}_{d}} \cong\left(1 \times a \phi_{d_{1}, d} b \phi_{d, d_{2}}\right)^{*} \mathcal{P}_{\mathcal{E}_{d}}=\left(1 \times a b c \phi_{d_{1}, d_{2}}\right)^{*} \mathcal{P}_{\mathcal{E}_{d}} \cong\left(\left(1 \times \phi_{d_{1}, d_{2}}\right)^{*} \mathcal{P}_{\mathcal{E}_{d}}\right)^{a b c}
$$

for a suitable integer $c$, so that the claim follows. (The first step here is essentially the definition of the dual isogeny.) 
Thus for each symmetric $Q \in \operatorname{End}\left(\prod_{i} \mathcal{E}_{d_{i}}\right)$, we obtain a line bundle $\hat{\mathcal{L}}_{Q ; d_{1}, \ldots, d_{n}}$ on $\prod_{i} \mathcal{E}_{d_{i}}$, and these line bundles satisfy the same compatibility relations as for our earlier construction on $\mathcal{E}^{n}$. In general, our two constructions do not agree, but there is one important special case.

Proposition 2.20. Suppose $Q \in \operatorname{Mat}_{n}(\mathbb{Z})$ is a symmetric matrix with even diagonal entries. Then $\hat{\mathcal{L}}_{Q ; 1, \ldots, 1}$ descends to $\mathcal{M}_{1,1}$, where it is canonically isomorphic to $\mathcal{L}_{Q, 0}$.

Proof. Any symmetric integer matrix with even diagonal is not only in the span of pullbacks of 1 , but in fact is in the span of pullbacks of $H=\left(\begin{array}{ll}0 & 1 \\ 1 & 0\end{array}\right)$, the symmetric endomorphism associated to the Poincaré bundle $\mathcal{P} \cong \hat{\mathcal{L}}_{H ; 1} \cong \mathcal{L}_{H, 0}$.

Remark. More generally, if $Q_{i i}$ is even whenever $d_{i}$ is odd, then $\hat{\mathcal{L}}_{Q ; \vec{d}}$ descends to $\mathcal{X}_{0}(2 N)$; similarly, if $Q_{i i}$ is even whenever $N / d_{i}$ is odd, then $\hat{\mathcal{L}}_{Q ; \vec{d}}$ descends to $\mathcal{X}_{0}(N, 2)$.

It will be convenient to have a somewhat more functorial version of the constructions of $\mathcal{E}^{n}$ or the products $\prod_{i} \mathcal{E}_{d_{i}}$ above. For the first, if $B$ is a finitely generated free abelian group, then we may consider the family of group schemes $\mathcal{E} \otimes B$. For a specific curve $E$, we may also construct $E \otimes B$, which is simply the corresponding fiber of $\mathcal{E} \otimes B$.

This clearly extends to a functor, which is exact on short exact sequences of free abelian groups. Note, however, that if we extend it in the obvious way to a functor on the category of all finitely generated abelian groups, then it is no longer exact. We readily compute the special cases

$$
\begin{aligned}
& E \otimes \mathbb{Z} \cong E, \\
& \operatorname{Tor}_{p}(E, \mathbb{Z})=0, \quad p>0, \\
& E \otimes \mathbb{Z} / N \mathbb{Z}=0, \\
& \operatorname{Tor}_{1}(E, \mathbb{Z} / N \mathbb{Z})=E[N], \\
& \operatorname{Tor}_{p}(E, \mathbb{Z} / N \mathbb{Z})=0, \quad p>0
\end{aligned}
$$

using the obvious projective resolution of $\mathbb{Z} / N \mathbb{Z}$. (This, of course, is the expected behavior for tensoring with a divisible group $E$.)

Proposition 2.21. Let $\phi: B \rightarrow C$ be a morphism of finitely generated free abelian groups. Then $E \otimes \phi$ is surjective iff $\operatorname{coker}(\phi)$ is finite, and injective iff $\operatorname{ker}(\phi)=0$ and $\operatorname{coker}(\phi)$ is free.

Proof. The four-term sequence

$$
0 \rightarrow \operatorname{ker} \phi \rightarrow B \rightarrow C \rightarrow \operatorname{coker} \phi \rightarrow 0
$$

is a free resolution, and thus we have an isomorphism $\operatorname{coker}(E \otimes \phi) \cong E \otimes \operatorname{coker} \phi$ and a short exact sequence

$$
0 \rightarrow E \otimes \operatorname{ker}(\phi) \rightarrow \operatorname{ker}(E \otimes \phi) \rightarrow \operatorname{Tor}_{1}(E, \operatorname{coker}(\phi)) \rightarrow 0 .
$$

The claim follows immediately.

Proposition 2.22. If $E$ does not have complex (or quaternionic) multiplication (in particular if $E=\mathcal{E})$, then $\operatorname{Hom}(B, C) \rightarrow \operatorname{Hom}(E \otimes B, E \otimes C)$ is an isomorphism.

Proof. This reduces to the case $B=\mathbb{Z}^{n}, C=\mathbb{Z}^{m}$, and thus to the case $B=C=\mathbb{Z}$, where it is essentially by definition.

Corollary 2.23. If $E$ does not have complex multiplication, then the natural map $B \rightarrow$ $\operatorname{Hom}(E, E \otimes B)$ is an isomorphism, as is the natural map $E \otimes \operatorname{Hom}(E, E \otimes B) \rightarrow E \otimes B$. 
The dual variety is then easy to compute.

Proposition 2.24. For $B$ free, there is a natural isomorphism $(E \otimes B)^{\vee} \cong E \otimes B^{*}$.

Proof. Indeed, we have $E \otimes B \cong E^{n}$, so dually $(E \otimes B)^{\vee} \cong E^{n}$. Thus for $E$ without complex multiplication, the natural map $E \otimes \operatorname{Hom}\left(E,(E \otimes B)^{\vee}\right) \rightarrow(E \otimes B)^{\vee}$ is an isomorphism. Duality gives $\operatorname{Hom}\left(E,(E \otimes B)^{\vee}\right) \cong \operatorname{Hom}(E \otimes B, E) \cong \operatorname{Hom}(B, \mathbb{Z})$, and thus $E \otimes \operatorname{Hom}(B, \mathbb{Z}) \cong(E \otimes B)^{\vee}$ as desired. The isomorphism holds for $E=\mathcal{E}$, and thus for all fibers $E$.

This gives the following description of the Néron-Severi group of $\mathcal{E} \otimes B: N S(\mathcal{E} \otimes B)$ consists of symmetric morphisms $\mathcal{E} \otimes B \rightarrow(\mathcal{E} \otimes B)^{\vee} \cong \mathcal{E} \otimes B^{*}$, and thus of symmetric pairings $Q: B \otimes B \rightarrow \mathbb{Z}$. We then find as above that any such symmetric pairing (and any weight) induces a line bundle $\mathcal{L}_{Q, w}$ on $\mathcal{E} \otimes B$.

Now, suppose $B \rightarrow C$ is an injective morphism with finite cokernel. Then we have a short exact sequence

$$
0 \rightarrow \operatorname{Tor}_{1}(\mathcal{E}, C / B) \rightarrow \mathcal{E} \otimes B \rightarrow \mathcal{E} \otimes C \rightarrow 0
$$

where the kernel is a product of groups of the form $E\left[d_{i}\right]$. If $C / B$ has exponent $N$, then we have $\operatorname{Tor}_{1}(\mathcal{E}, C / B) \cong \mathcal{E}[N] \otimes_{\mathbb{Z} / N \mathbb{Z}} C / B$, which in turn suggests that we consider the subgroup $\kappa_{N} \otimes_{\mathbb{Z} / N \mathbb{Z}} C / B$, where $\kappa_{N}$ is the kernel of a the cyclic $N$-isogeny corresponding to a point of $\mathcal{X}_{0}(N)$. This, it turns out, does not quite behave correctly in characteristic dividing $N$, but we do have the following.

Proposition 2.25. Let $N$ be a positive integer, and let $B$ be a finitely generated abelian group of exponent $N$. Then the group scheme $\kappa_{N} \otimes_{\mathbb{Z} / N \mathbb{Z}} B$ on $\mathcal{X}_{0}(N) \times \operatorname{Spec}(\mathbb{Z}[1 / N])$ extends in a natural way to a flat group scheme on $\mathcal{X}_{0}(N)$.

Proof. If we choose an isomorphism $B \cong \bigoplus \mathbb{Z} / d_{i} \mathbb{Z}$, then we certainly have such an extension: away from $N$, the group is just $\prod_{i} \kappa_{d_{i}}$, where $\kappa_{d_{i}}$ is the kernel of the isogeny $E_{1} \rightarrow E_{d_{i}}$, and this product makes sense in all characteristics. If $B \rightarrow B^{\prime}$ is a morphism of $N$-torsion groups, then the morphism $\kappa_{N} \otimes_{\mathbb{Z} / N \mathbb{Z}} B \rightarrow \kappa_{N} \otimes_{\mathbb{Z} / N \mathbb{Z}} B^{\prime}$ is simply the restriction of the morphism $E[N] \otimes_{\mathbb{Z} / N \mathbb{Z}} B \rightarrow E[N] \otimes_{\mathbb{Z} / N \mathbb{Z}} B^{\prime}$. The latter morphism is defined in all characteristics, and the requirement that it restrict to a specific morphism is a closed condition, so is inherited from the generic case.

This allows us to define families of abelian varieties over $\mathcal{X}_{0}(N)$ as follows: given abelian groups $B, C$ such that $N C \subset B \subset C$, we define $\mathcal{E}_{B, C}$ to be the quotient of $\mathcal{E} \otimes B$ by the subgroup scheme extending $\kappa_{N} \otimes_{\mathbb{Z} / N \mathbb{Z}} C / B$. (We will denote this extension by the same tensor product notation, but caution the reader that it is not the actual tensor product in general.)

Proposition 2.26. If $N C_{1} \subset B_{1} \subset C_{1}, N C_{2} \subset B_{2} \subset C_{2}$ are pairs of finitely generated free abelian groups and $\phi: C_{1} \rightarrow C_{2}$ is a morphism such that $\phi\left(B_{1}\right) \subset B_{2}$, then there is an induced morphism $\mathcal{E}_{B_{1}, C_{1}} \rightarrow \mathcal{E}_{B_{2}, C_{2}}$, making the construction functorial.

Proof. The condition on $\phi$ implies that it induces a morphism $C_{1} / B_{1} \rightarrow C_{2} / B_{2}$, and thus we have a commutative diagram

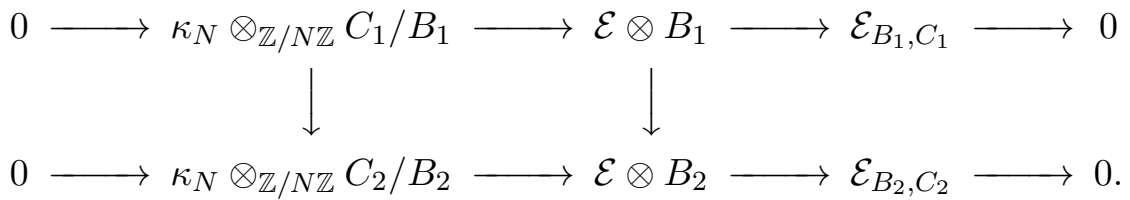

Since the rows are exact and the given vertical arrows are functorial, the claim follows. 
Of course, given an isomorphism $C / B \cong \prod_{i} \mathbb{Z} / d_{i} \mathbb{Z}$, there is a corresponding isomorphism $\mathcal{E}_{B, C} \cong \prod_{i} \mathcal{E}_{d_{i}}$. This makes the following straightforward to verify.

Proposition 2.27. If $E_{1} \rightarrow E_{N}$ is a cyclic $N$-isogeny between curves with no complex multiplication, then the morphisms between corresponding fibers of $\mathcal{E}_{B_{1}, C_{1}}$ and $\mathcal{E}_{B_{2}, C_{2}}$ are precisely those coming from the previous proposition.

Corollary 2.28. For $N C \subset B \subset C$, we have

$$
\begin{aligned}
& \operatorname{Hom}\left(\mathcal{E}, \mathcal{E}_{B, C}\right) \cong B, \\
& \operatorname{Hom}\left(\mathcal{E}_{N}, \mathcal{E}_{B, C}\right) \cong C
\end{aligned}
$$

in such a way that composition with the dual isogeny $\mathcal{E}_{N} \rightarrow \mathcal{E}$ induces the inclusion $B \subset C$.

Proposition 2.29. There is a natural isomorphism $\mathcal{E}_{B, C}^{\vee} \cong \mathcal{E}_{C^{*}, B^{*}}$.

Proof. The previous corollary allows us to canonically identify anything isomorphic to a product of curves $\mathcal{E}_{d_{i}}$ with a variety of the form $\mathcal{E}_{B, C}$. Since $\mathcal{E}_{B, C}$ is isomorphic to such a product, its dual is also of that form, and thus it remains only to compute $\operatorname{Hom}\left(\mathcal{E}, \mathcal{E}_{B, C}^{\vee}\right)$ and $\operatorname{Hom}\left(\mathcal{E}_{N}, \mathcal{E}_{B, C}^{\vee}\right)$.

It follows that the Néron-Severi group of $\mathcal{E}_{B, C}$ (or of any fiber without complex multiplication) consists of pairings $B \otimes C \rightarrow \mathbb{Z}$ which become symmetric when restricted to $B \otimes B$; equivalently, it consists of symmetric pairings $Q: B \otimes B \rightarrow \mathbb{Z}$ such that $Q(B, N C) \subset N \mathbb{Z}$. From our construction above, we find that if we base change to $\mathcal{X}_{0}(2 N, 2)$, then any such symmetric pairing induces a natural line bundle $\hat{\mathcal{L}}_{Q ; B, C}$ on $\mathcal{E}_{B, C}$, satisfying the appropriate compatibility relations.

Suppose now that $Q$ is a positive definite pairing of "level" dividing $N$ (i.e., such that $\left.N B^{*} \subset Q B\right)$ Then we have a chain of free abelian groups $Q^{-1} N B^{*} \subset B \subset C \subset Q^{-1} B^{*}$, giving rise to an isogeny $\pi: \mathcal{E}_{B, C} \rightarrow \mathcal{E}_{B, Q^{-1} B^{*}}$. The pairing $Q$ still induces an element of the NéronSeveri group of the quotient, which by degree considerations is now a principal polarization, represented by the line bundle $\hat{\Theta}_{B}:=\hat{\mathcal{L}}_{Q ; B, Q^{-1} B^{*}}$. We moreover find that $\pi^{*} \hat{\Theta}_{B} \cong \hat{\mathcal{L}}_{Q ; B, C}$.

We also note that the action of $\mathcal{E}[N] \otimes\left(Q^{-1} B^{*} / B\right) \cong \operatorname{Tor}_{1}\left(\mathcal{E}, Q^{-1} B^{*} / B\right)$ on $\mathcal{E} \otimes B$ descends to an action of the quotient $\left(\mathcal{E}[N] \otimes\left(Q^{-1} B^{*} / B\right)\right) /\left(\kappa_{N} \otimes\left(Q^{-1} B^{*} / B\right)\right)$ on $\mathcal{E}_{B, Q^{-1} B^{*}}$, which by the Weil pairing may be identified with an action of the Pontryagin dual $\operatorname{Hom}\left(\kappa_{N} \otimes\right.$ $\left.\left(Q^{-1} B^{*} / B\right), \mu_{N}\right)$. If $\kappa_{N}$ is diagonalizable, then this dual is discrete, and may be identified with $\operatorname{Hom}\left(Q^{-1} B^{*} / B, \operatorname{Hom}\left(\kappa_{N}, \mu_{N}\right)\right)$.

Lemma 2.30. On the locus of $\mathcal{X}_{0}(2 N, 2)$ where the $N$-isogeny $E_{1} \rightarrow E_{N}$ has diagonalizable kernel $\kappa_{N}$, we have natural identifications

$$
\Gamma\left(E_{B, C} ; \hat{\mathcal{L}}_{Q ; B, C}\right) \cong \bigoplus_{g \in \operatorname{Hom}\left(Q^{-1} B^{*} / C, \operatorname{Hom}\left(\kappa_{N}, \mu_{N}\right)\right)} g^{*} \Gamma\left(E_{B, Q^{-1} B^{*}} ; \hat{\Theta}_{B}\right),
$$

in which each (1-dimensional!) summand on the right is an eigenspace for the induced action of $\operatorname{ker}\left(E_{B, C} \rightarrow E_{B, Q^{-1} B^{*}}\right)$.

Proof. We have

$$
\Gamma\left(E_{B, C} ; \hat{\mathcal{L}}_{Q ; B, C}\right) \cong \Gamma\left(E_{B, C} ; \pi^{*} \hat{\Theta}_{B}\right) \cong \Gamma\left(E_{B, Q^{-1} B^{*}} ; \pi_{*} \pi^{*} \hat{\Theta}_{B}\right)
$$

The natural map $\hat{\Theta}_{B} \rightarrow \pi_{*} \pi^{*} \hat{\Theta}_{B}$ selects a particular eigenspace of the kernel of the isogeny, and the decomposition as claimed then follows by the structure theory of representations of Heisenberg groups, see [32] as well as the exposition in [22]. Here we use the fact that $\Gamma\left(E_{B, C} ; \hat{\mathcal{L}}_{Q ; B, C}\right)$ is the unique irreducible representation of the Heisenberg group $\mathcal{G}\left(\hat{\mathcal{L}}_{Q ; B, C}\right)$ on which the central $\mathbb{G}_{m}$ acts with weight 1, together with the fact that the commutator pairing on the Heisenberg group is precisely the Weil pairing, so the different isotypic components for the diagonalizable kernel are related via the complementary translation subgroup. 
Corollary 2.31. Suppose that the finite group $G$ acts on $C$, preserving the subgroup $B$ and the polarization $Q: B \rightarrow B^{*}$ so that $G$ acts on $\mathcal{E}_{B, C}$ as automorphisms fixing the identity, with an induced equivariant structure on $\hat{\mathcal{L}}_{Q ; B, C}$. On the locus of $\mathcal{X}_{0}(2 N, 2)$ where $E_{1} \rightarrow E_{N}$ has diagonalizable kernel, the $G$-module $\Gamma\left(E_{B, C} ; \hat{\mathcal{L}}_{Q ; B, C}\right)$ is isomorphic to the permutation module arising from the action of $G$ on $\operatorname{Hom}\left(Q^{-1} B^{*} / C, \mathbb{Q} / \mathbb{Z}\right)$.

Remark. Here we note that for general free abelian groups $B \subset C$ with finite quotient,

$$
\operatorname{Hom}(C / B, \mathbb{Q} / \mathbb{Z}) \cong \operatorname{Tor}_{1}\left(B^{*} / C^{*}, \mathbb{Q} / \mathbb{Z}\right) \cong B^{*} / C^{*},
$$

and thus

$$
\operatorname{Hom}\left(Q^{-1} B^{*} / C, \mathbb{Q} / \mathbb{Z}\right) \cong C^{*} / Q B \cong Q^{-1} C^{*} / B .
$$

Corollary 2.32. Let $B$ be a finitely generated free abelian group and $Q: B \otimes B \rightarrow \mathbb{Z}$ an even symmetric pairing of level dividing $N$, and let $E$ be an elliptic curve which, if supersingular, has characteristic prime to $N$. Then $\Gamma\left(E \otimes B ; \mathcal{L}_{Q, 0}\right)$ is isomorphic as a $G$-module to the permutation module coming from the action of $G$ on $Q^{-1} B^{*} / B$.

Proof. The condition on $E$ ensures that we may choose a point of $\mathcal{X}_{0}(2 N, 2)$ lying over it such that the cyclic $N$-isogeny $E \cong E_{1} \rightarrow E_{N}$ has diagonalizable kernel. We may then identify the $G$-module $\Gamma\left(E \otimes B ; \mathcal{L}_{Q, 0}\right)$ with $\Gamma\left(E_{B, B} ; \hat{\mathcal{L}}_{Q ; B, B}\right)$ and thus apply the previous corollary.

Corollary 2.33. With the same hypotheses, the dimension $\operatorname{dim}\left(\Gamma\left(E \otimes B ; \mathcal{L}_{Q, 0}\right)^{G}\right)$ is equal to the number of orbits of $G$ in $Q^{-1} B^{*} / B$.

Remark. Both conclusions remain valid for supersingular curves of characteristic prime to $|G|$; indeed, any 1-parameter family of $G$-modules over an algebraically closed field containing $1 /|G|$ is trivial, so the claims follow from the case of ordinary curves.

It turns out that the exclusion of certain supersingular curves above is indeed necessary. For instance, suppose that $B=\mathbb{Z}^{8}$ and $Q$ is the Gram matrix of the lattice $Q_{8}(1)$ of [5]. This is a symmetric matrix with even diagonal and elementary divisors $1,1,1,1,5,5,5$, 5, and the corresponding automorphism group $\mathrm{GO}_{4}^{+}(5)$ acts in the natural way on $\operatorname{coker}(Q)$. If $E$ is the (geometrically unique) supersingular curve of characteristic 5, then one finds that the induced $\mathrm{GO}_{4}^{+}(5)$-module structure on $\Gamma\left(E^{8} ; \mathcal{L}_{Q, 0}\right)$ is not isomorphic to the given permutation representation. In this case, the actual invariant subspace does not jump, but we find that

$$
\operatorname{dim}\left(\Gamma\left(E^{16} ; \mathcal{L}_{Q \oplus Q, 0}\right)^{\mathrm{GO}_{4}^{+}(5)}\right) \cong \operatorname{dim}\left(\left(\Gamma\left(E^{8} ; \mathcal{L}_{Q, 0}\right)^{\otimes 2}\right)^{\mathrm{GO}_{4}^{+}(5)}\right)=160,
$$

while for all other curves, the invariant space has dimension 156 . To compute the action of $G$ in such supersingular cases, we may use the fact that $\mathcal{L}_{Q, 0}$ always descends to the appropriate quotient and gives an equivariant isomorphism $\Gamma\left(E^{n} ; \mathcal{L}_{Q, 0}\right) \cong \Gamma\left(\operatorname{ker} \psi_{Q}^{\vee} ; \mathcal{L}^{\prime}\right)$; what fails is that we no longer have a natural basis (which in the case of the theorem are an eigenbasis for the action of the diagonalizable group ker $\left.\psi_{Q}\right)$ of the latter. There is, in fact, an isomorphism

$$
\Gamma\left(\operatorname{ker} \psi_{Q}^{\vee} ; \mathcal{L}^{\prime}\right) \cong \Gamma\left(\operatorname{ker} \psi_{Q}^{\vee} ; \mathcal{O}_{\operatorname{ker} \psi_{Q}^{\vee}}\right),
$$

since $\operatorname{ker} \psi_{Q}^{\vee}$ is 0-dimensional, but this is not in general equivariant; in general, the action of $G$ is twisted by some cocycle with values in the unit group of the coordinate $\operatorname{ring}$. When $\operatorname{det}(Q)$ is odd, however, we can use the description of $\mathcal{L}^{\prime}$ in terms of pullbacks of $\mathcal{O}_{\mathcal{E}_{d}}\left(\left[\sigma_{d}\right]\right)$ to compute this cocycle. In the $Q_{8}(1)$ case, this further simplifies, since we only need to know what happens on $\alpha_{5}^{4}$, allowing us to reduce to an evaluation of functions on $E_{5}^{4}$ in an appropriate formal neighborhood of the identity. 
A similar calculation applies to the Gram matrix of $\sqrt{3} \Lambda_{E_{6}}^{\perp}$, with its automorphism group $O_{5}(3)$; in this case, there are also subgroups of $O_{5}(3)$ for which the corollary fails on the supersingular curve of characteristic 3 . We can also obtain a characteristic 2 counterexample from $\sqrt{2} \Lambda_{E_{7}}^{\perp}$; in this case, it is unclear how to compute the cocycle, but we can simply check that none of the 16 elements of $H^{1}\left(\operatorname{Sp}_{6}(2) ; \mu_{2}\left(\alpha_{2}^{6}\right)\right)$ give rise to the permutation module. (There is also a subgroup with too many invariants, namely the preimage in $W\left(D_{6}\right)$ of the transitive Alt $_{5} \subset S_{6}$, which has too many invariants in each of the 16 possible cases.)

We should further note that the requirement that $Q$ have even diagonal is also necessary; indeed, otherwise the claim already fails for the case $Q=1, G=\mathrm{GL}_{1}(\mathbb{Z})$ for any curve of characteristic not 2 .

Even when $E$ is supersingular of characteristic dividing $N$, there may still be isogenies of the form

$$
E_{B, C} \rightarrow E_{B, C^{\prime}}
$$

with diagonalizable kernel, which as an abstract group scheme can be (geometrically) identified with $\mu_{N} \otimes_{\mathbb{Z} / N \mathbb{Z}} C^{\prime} / C$. Indeed, the only requirement is that $\left|C^{\prime} / C\right|$ be prime to the characteristic of $E$. Making this a canonical identification is somewhat trickier, as the kernel is only naturally described as the quotient

$$
\left(\kappa_{N} \otimes_{\mathbb{Z} / N \mathbb{Z}} C^{\prime} / B\right) /\left(\kappa_{N} \otimes_{\mathbb{Z} / N \mathbb{Z}} C / B\right) .
$$

Moreover, the translations moving between the different eigenspaces are only defined up to the kernel of the descended polarization. We find in general that

$$
\Gamma\left(E_{B, C} ; \mathcal{L}_{Q ; B, C}\right) \cong \bigoplus_{g} g^{*} \Gamma\left(E_{B, C^{\prime}} ; \mathcal{L}_{Q ; B, C^{\prime}}\right)
$$

where $g$ ranges over the quotient group

$$
\operatorname{Hom}\left(Q^{-1} B^{*} / C, \operatorname{Hom}\left(\kappa_{N}, \mu_{N}\right)\right) / \operatorname{Hom}\left(Q^{-1} B^{*} / C^{\prime}, \operatorname{Hom}\left(\kappa_{N}, \mu_{N}\right)\right) .
$$

We can thus only use this decomposition in understanding group actions when this quotient group has an equivariant splitting. Luckily, there is an important case when this happens: if $C^{\prime} / C$ is the $l$-part of $Q^{-1} B^{*} / C$ for some prime $l$ which is invertible on $E$, then the quotient may be identified with the $l$-part of $\operatorname{Hom}\left(Q^{-1} B^{*} / C, \operatorname{Hom}\left(\kappa_{N}, \mu_{N}\right)\right)$.

Lemma 2.34. With $B, C, Q$ as above and $l$ a prime invertible on $E$, there is a $G$-equivariant isomorphism

$$
\Gamma\left(E_{B, C} ; \mathcal{L}_{Q ; B, C}\right) \cong \bigoplus_{H} \operatorname{Ind}_{H}^{G} \operatorname{Res}_{H}^{G} \Gamma\left(E_{B, C^{\prime}} ; \mathcal{L}_{Q ; B, C^{\prime}}\right),
$$

where $H$ ranges over the point stabilizers in the different orbits of the action of $G$ on the l-part of $Q^{-1} C^{*} / B$ and

$$
C^{\prime}=\bigcup_{k} Q^{-1} B^{*} \cap l^{-k} C
$$

Here we should note that we have such a reduction for every prime dividing $N$; in particular, if the polarization does not have prime power degree, then we can always choose a prime dividing the degree of the polarization which is invertible on $E$, and use the corresponding reduction.

Although we have seen that there can indeed be (finitely many) bad curves for such invariant theory questions, it turns out that our hypotheses are in fact slightly more restrictive than 
they need to be. Suppose we have a finite group $G$ acting on an abelian variety $A$ (fixing the identity). There are two natural induced abelian subvarieties. The subgroup scheme $A^{G}$ is still projective, and thus (up to a possible inseparable base change) we may take its reduced identity component $A^{G 0}$. Equivalently (and without need for base change), we could instead define $A^{G 0}$ to be the image of the endomorphism $\sum_{g \in G} g \in \operatorname{End}(A)$. There is also an almost complementary subvariety $A_{G}$ giving by the image of the endomorphism $|G|-\sum_{g \in G} g$. Both subvarieties are clearly preserved by $G$, and since the sum of the endomorphisms is an isogeny, it follows that we have a natural $G$-equivariant isogeny $A_{G} \times A^{G 0} \rightarrow A$. Any $G$-equivariant line bundle on $A$ (with trivial action on the fiber over the identity) pulls back to a $G$-equivariant line bundle on $A_{G} \times A^{G 0}$, namely $\left.\left.\mathcal{L}\right|_{A_{G}} \otimes \mathcal{L}\right|_{A^{G 0}}$. Moreover, the action of $G$ on the second factor is trivial, since it is trivial at the identity.

It turns out that even though this isogeny can fail to have diagonalizable kernel, we can still use it to reduce questions about $G$-module structures to $A_{G}$.

Lemma 2.35. With $A, G, \mathcal{L}$ as above, assume that $\mathcal{L}$ is ample. Then there is a $G$-module isomorphism

$$
\Gamma(A ; \mathcal{L})^{d} \cong \Gamma\left(A_{G} ;\left.\mathcal{L}\right|_{A_{G}}\right)^{e},
$$

where $d=\left|A_{G} \cap A^{G 0}\right|$ and $e=\operatorname{dim} \Gamma\left(A^{G 0} ;\left.\mathcal{L}\right|_{A^{G 0}}\right)$.

Proof. Let $K=A_{G} \cap A^{G 0}$ be the kernel of the isogeny $A_{G} \times A^{G 0} \rightarrow A$. Then we have a natural isomorphism

$$
\Gamma(A ; \mathcal{L}) \cong \Gamma\left(A_{G} \times A^{G 0} ;\left.\left.\mathcal{L}\right|_{A_{G}} \otimes \mathcal{L}\right|_{A^{G 0}}\right)^{K} \cong\left(\Gamma\left(A_{G} ;\left.\mathcal{L}\right|_{A_{G}}\right) \otimes \Gamma\left(A^{G 0} ;\left.\mathcal{L}\right|_{A^{G 0}}\right)\right)^{K} .
$$

Let $H$ be the preimage of $K$ in the Heisenberg group $\mathcal{G}\left(\mathcal{L}_{A^{G 0}}^{-1}\right)$. This certainly acts naturally on $\Gamma\left(A^{G 0} ;\left.\mathcal{L}\right|_{A^{G 0}}\right)^{*}$, but the fact that the line bundle descends to $A$ implies that it also acts on $\Gamma\left(A_{G} ;\left.\mathcal{L}\right|_{A_{G}}\right)$. We thus have a natural isomorphism

$$
\left(\Gamma\left(A_{G} ;\left.\mathcal{L}\right|_{A_{G}}\right) \otimes \Gamma\left(A^{G 0} ;\left.\mathcal{L}\right|_{A^{G 0}}\right)\right)^{K} \cong \operatorname{Hom}_{H}\left(\Gamma\left(A^{G 0} ;\left.\mathcal{L}\right|_{A^{G 0}}\right)^{*}, \Gamma\left(A_{G} ;\left.\mathcal{L}\right|_{A_{G}}\right)\right),
$$

which by Frobenius reciprocity further becomes

$$
\begin{aligned}
& \operatorname{Hom}_{H}\left(\Gamma\left(A^{G 0} ;\left.\mathcal{L}\right|_{A^{G 0}}\right)^{*}, \Gamma\left(A_{G} ;\left.\mathcal{L}\right|_{A_{G}}\right)\right) \\
& \cong \operatorname{Hom}_{\mathcal{G}\left(\left.\mathcal{L}\right|_{A} ^{-1}\right)}\left(\Gamma\left(A^{G 0} ;\left.\mathcal{L}\right|_{A^{G 0}}\right)^{*}, \operatorname{Ind}_{H} \mathcal{G}\left(\mathcal{L}_{A}^{-1}\right) \Gamma\left(A_{G} ;\left.\mathcal{L}\right|_{A_{G}}\right)\right) \text {. }
\end{aligned}
$$

By the structure of Heisenberg representations, we may then conclude that there is a functorial isomorphism

$$
\Gamma(A ; \mathcal{L}) \otimes \Gamma\left(A^{G 0} ;\left.\mathcal{L}\right|_{A}{ }^{G 0}\right) \cong \operatorname{Ind}_{H}^{\mathcal{G}\left(\mathcal{L}_{A}^{-1}{ }^{G 0}\right.} \Gamma\left(A_{G} ;\left.\mathcal{L}\right|_{A_{G}}\right)
$$

of $\mathcal{G}\left(\mathcal{L}_{A G 0}^{-1}\right)$-modules. Moreover, the splitting $K \rightarrow H$ is $G$-invariant, since it could be computed inside $A^{G 0}$, and thus we may rewrite this as a $G \times \mathcal{G}\left(\mathcal{L}_{A^{G 0}}^{-1}\right)$-module isomorphism:

$$
\Gamma(A ; \mathcal{L}) \otimes \Gamma\left(A^{G 0} ;\left.\mathcal{L}\right|_{A^{G 0}}\right) \cong \operatorname{Ind}_{G \times H}^{G \times \mathcal{G}\left(\mathcal{L}_{A}^{-1}\right)} \Gamma\left(A_{G} ;\left.\mathcal{L}\right|_{A_{G}}\right) .
$$

Moreover, the induction functor is exact (since the homogeneous space is affine), as is restriction to $G$, and thus if we forget the action of the Heisenberg group, we obtain a $G$-module isomorphism

$$
\Gamma(A ; \mathcal{L})^{e} \cong \Gamma\left(A_{G} ;\left.\mathcal{L}\right|_{A_{G}}\right)^{e^{2} / d},
$$

from which the claim follows.

Remark 2.36. Note that although $d$ always divides $e^{2}$, it need not divide $e$, and vice versa $e$ can fail to divide $d$.

Remark 2.37. The reader should note that the notion of an induced module for representations of group schemes corresponds to what would normally be called a coinduced module. 


\section{Coxeter group actions on abelian varieties}

One of the major ingredients in the construction of elliptic analogues of double affine Hecke algebras is a suitable action of an affine Weyl group on a power of an elliptic curve (or more generally on a variety isogenous to such a power). It will be convenient to work somewhat more abstractly, and begin with the finite case.

With this in mind, let $A$ be an abelian variety, and suppose the finite Weyl group $W$ acts faithfully on $A$ (fixing the identity) in such a way that for any reflection $r \in R(W)$, the corresponding fixed subgroup scheme has codimension 1 . We will naturally refer to such an action as an action "by reflections".

For each reflection $r \in R(W)$, the subgroup $\langle r\rangle$ splits $A$ (up to isogeny) as discussed above; in this case, we have natural subvarieties $A^{r 0}:=\operatorname{im}(1+r)$ and $A_{r}:=\operatorname{im}(1-r)$, and an induced isogeny $A^{r 0} \times A_{r} \rightarrow A$ (with kernel contained in $A_{r}[2]$ ). Since $A^{r 0}$ by assumption has codimension 1 , we see that $A_{r}$ is a 1-dimensional abelian variety. In other words, each reflection in $W$ induces a corresponding elliptic curve $E_{r}=A_{r}$ contained in $A$, as the image of the endomorphism $1-r$. We call such a curve the "root curve" associated to $r$. Applying the same construction to the dual variety $A^{\vee}$ gives root curves $E_{r}^{\prime} \subset A^{\vee}$, the duals of which we refer to as "coroot curves". Note that the coroot curve associated to $r$ can be described directly as the cokernel of the endomorphism $1+r$. In particular, the endomorphism $1-r$ factors through $E_{r}^{\prime}$, giving rise to a natural map $E_{r}^{\prime} \rightarrow E_{r}$ such that the composition $A \rightarrow E_{r}^{\prime} \rightarrow E_{r} \rightarrow A$ is $1-r$ and the composition $E_{r} \rightarrow A \rightarrow E_{r}^{\prime} \rightarrow E_{r}$ is multiplication by 2 .

Fix a system of simple roots $S=\left\{\alpha_{1}, \ldots, \alpha_{n}\right\}$ in $W$, and let $E_{1}, \ldots, E_{n} ; E_{1}^{\prime}, \ldots, E_{n}^{\prime}$ be the corresponding root and coroot curves, with induced maps $\iota_{i}: E_{i} \rightarrow A, \iota_{i}^{\prime}: A \rightarrow E_{i}^{\prime}, \nu_{i}: E_{i}^{\prime} \rightarrow E_{i}$. The action of $s_{i}$ on $E_{j}$ can be described quite simply:

$$
s_{i} \circ \iota_{j}=\iota_{j}+\left(s_{i}-1\right) \circ \iota_{j}=\iota_{j}-\iota_{i} \circ \nu_{i} \circ \iota_{i}^{\prime} \circ \iota_{j} .
$$

This suggests that we should define a morphism $\mu_{i j}: E_{j} \rightarrow E_{i}$ as the composition $-\nu_{i} \circ \iota_{i}^{\prime} \circ \iota_{j}$; that is, it is the morphism $E_{j} \rightarrow E_{i}$ induced by $s_{i}-1$.

Lemma 3.1. The curves $E_{1}, \ldots, E_{n}$ are distinct.

Proof. Suppose otherwise, and reorder the simple roots so that $E_{1}=E_{2}$. Then $s_{1} s_{2} \neq 1$, but

$$
\left(s_{1} s_{2}-1\right)=\left(s_{1}-1\right)\left(s_{2}-1\right)+\left(s_{1}-1\right)+\left(s_{2}-1\right)
$$

so that $s_{1} s_{2}-1$ has image $E_{1}=E_{2}$. Since $s_{1} s_{2}$ fixes $E_{1}=E_{2}$, we find that $\left(s_{1} s_{2}\right)^{k}-1=k\left(s_{1} s_{2}-1\right)$ for all $k \geq 1$, and thus $s_{1} s_{2}$ has infinite order, contradicting finiteness of $W$.

Lemma 3.2. For $i \neq j$, the composition $\mu_{j i} \circ \mu_{i j}$ is multiplication by $k \in\{0,1,2,3\}$, and if the composition is 0 , then $\mu_{i j}=\mu_{j i}=0$.

Proof. Since $E_{i} \neq E_{j}$, the product $E_{i} \times E_{j}$ is isogenous with its image in $A$. Define an action of $s_{i}, s_{j}$ on $E_{i} \times E_{j}$ by

$$
\begin{aligned}
& s_{i}\left(x_{i}, x_{j}\right)=\left(-x_{i}+\mu_{j i}\left(x_{j}\right), x_{j}\right), \\
& s_{j}\left(x_{i}, x_{j}\right)=\left(x_{i},-x_{j}+\mu_{i j}\left(x_{i}\right)\right) .
\end{aligned}
$$

The elements $s_{i}, s_{j}$ clearly act as involutions on the product, and the actions are compatible with the actions on $A$, so that the action of $\left(s_{i} s_{j}\right)^{m_{i j}}-1$ induces a homomorphism from $E_{i} \times E_{j}$ to the kernel of the map $E_{i} \times E_{j} \rightarrow A$. Since $E_{i} \times E_{j}$ is proper, reduced, and connected, and said kernel is finite, we see that this description actually gives an action of the rank 2 Weyl 
group $\left\langle s_{i}, s_{j}\right\rangle$. Moreover, since this group is finite, there is a $W$-invariant polarization on $E_{i} \times E_{j}$, of the form

$$
\left(\begin{array}{cc}
2 r_{i} & -\psi_{j i} \\
-\psi_{j i}^{\vee} & 2 r_{j}
\end{array}\right)
$$

with $4 r_{i} r_{j}-\operatorname{deg}\left(\psi_{j i}\right)>0$. We then find that $\psi_{j i}=r_{i} \mu_{j i}=r_{j} \mu_{i j}^{\vee}$, so that

$$
\operatorname{deg}\left(\psi_{j i}\right)=\psi_{j i} \psi_{j i}^{\vee}=r_{i} \mu_{j i} r_{j} \mu_{i j}
$$

and thus $\mu_{j i} \mu_{i j}$ is multiplication by a nonnegative integer less than 4 . Moreover, since $r_{i} \mu_{j i}=$ $r_{j} \mu_{i j}^{\vee}$, we see that if one of $\mu_{i j}, \mu_{j i}$ vanishes, then so does the other.

Remark. We then readily see that the order of $s_{i} s_{j}$ is equal to $2,3,4,6$ when $\mu_{i j} \mu_{j i}=\mu_{j i} \mu_{i j}$ is equal to $0,1,2,3$ respectively.

Proposition 3.3. Let $(W, S)$ be a finite Weyl group, and suppose that $E_{1}, \ldots, E_{n}$ is a system of elliptic curves and $\mu_{i j}: E_{i} \rightarrow E_{j}, i \neq j$, a system of morphisms such that $\mu_{i j} \mu_{j i}=4 \cos \left(\pi / m_{i j}\right)^{2}$, with $\mu_{i j}=\mu_{j i}=0$ whenever $m_{i j}=2$. Then there is a faithful action of $W$ on $\prod_{i} E_{i}$ such that

$$
s_{i}\left(x_{1}, \ldots, x_{n}\right)=\left(x_{1}, \ldots, x_{i-1},-x_{i}+\sum_{j \neq i} \mu_{j i}\left(x_{j}\right), x_{i+1}, \ldots, x_{n}\right) .
$$

Proof. The action of $s_{i}$ is clearly an involution, and the braid relations are straightforward to verify. (This is easy when $m_{i j}=2$, and for $m_{i j}>2$ it suffices to show that $\left(\left(s_{i} s_{j}\right)^{2}+\right.$ $\left.\left(2-\mu_{i j} \mu_{j i}\right)\left(s_{i} s_{j}\right)+1\right)\left(s_{i} s_{j}-1\right)$ vanishes, which reduces to a computation in $E_{i} \times E_{j}$.) So this certainly gives an action of $W$, and it remains only to show that it is faithful.

Since the construction clearly respects products, we may as well assume that $W$ is irreducible. For any path in the Coxeter diagram of $W$, we may take the corresponding composition of morphisms $\mu_{i j}$; since $\mu_{i j}=0$ iff $m_{i j}=2$, any such composition will be an isogeny. Moreover, since the Coxeter diagram is a tree by finiteness of $W$, we see that any two isogenies $E_{i} \rightarrow E_{j}$ arising in this way will differ by a positive factor (any time the path backtracks introduces a factor $\mu_{i j} \mu_{j i}>0$ ). In particular, for any element $w \in W$, the induced map $E_{i} \rightarrow E_{j}$ (apply $w$ then project onto the $j$ th factor) is an integer linear combination of such isogenies, and in particular has a corresponding notion of positivity. This allows us to turn any element of $W$ into a real matrix by taking each such morphism to the appropriately signed square root of its degree. The consistency of sign ensures that this will give rise to an actual representation of $W$, and we can then verify that up to a diagonal change of basis, this is precisely the standard reflection representation of $W$.

Corollary 3.4. Let the finite Weyl group $W$ act faithfully on the abelian variety $A$ by reflections, with simple root curves $E_{1}, \ldots, E_{n}$. Then the induced morphism $\prod_{i} E_{i} \rightarrow A$ is made $W$-equivariant by the above action, and its kernel is finite and fixed by $W$.

Proof. The equivariance is obvious by construction, so it remains only to show that the kernel $K$ is fixed by $W$. Otherwise, some simple reflection $s_{i}$ will act nontrivially on $K$, and thus $\left(s_{i}-1\right) K$ $\subset K$ is nonzero. But $\left(s_{i}-1\right) K \subset E_{i}$, and $K \cap E_{i}=0$ since $E_{i}$ is defined as a subscheme of $A$.

The proof of the proposition suggests an extension of this construction to more general crystallographic Coxeter groups (in particular to affine Weyl groups). Certainly, one could consider an action of the above form associated to any system of morphisms $\mu_{i j}$, but to relate it to the standard reflection representation of a Coxeter group, we make the following assumptions: 
- The composition $\mu_{i j} \mu_{j i}$ is multiplication by $k_{i j} \in\{0,1,2,3,4\}$.

- There is a system of positive integers $r_{i}$ such that $r_{i} \mu_{j i}=r_{j} \mu_{i j}^{\vee}$ for each $i \neq j$.

- Any composition $\mu_{i_{1} i_{2}} \mu_{i_{2} i_{3}} \cdots \mu_{i_{m} i_{1}}$ is multiplication by a nonnegative integer.

We call such a system of curves and morphisms an "elliptic root datum".

Theorem 3.5. Any elliptic root datum gives rise to a faithful action on $\prod_{i} E_{i}$ of the Coxeter group with multiplicities $m_{i j}$ given by $k_{i j}=4 \cos \left(\pi / m_{i j}\right)^{2}$, such that $s_{i}$ acts as above.

Proof. The conditions on the morphisms ensure that we can faithfully translate the action into one on a real vector space, taking each morphism to the appropriately signed square root of its degree. Conjugating by the diagonal matrix with entries $\sqrt{r_{i}}$ turns this into the standard reflection representation of the given Coxeter group.

Corollary 3.6. For each conjugacy class $C$ of reflections, there is a corresponding elliptic curve $E_{C}$ equipped with isomorphisms $E_{C} \cong E_{r}, r \in C$ such that the action of $W$ on the set of compositions $E_{C} \cong E_{r} \rightarrow A$ and their negatives can be identified with the action of $W$ on the corresponding set of roots, with the compositions $E_{C} \cong E_{r} \rightarrow A$ corresponding to the positive roots.

Proof. Indeed, for each simple root $\alpha_{i}$, we may consider the set of compositions $w \circ \iota_{i}$ for $w \in W$, and find that each such composition has the form $\left(\beta_{i 1}, \beta_{i 2}, \ldots, \beta_{i n}\right)$ in which either every entry is a nonnegative element of the relevant Hom space (i.e., corresponding to a nonnegative real number) or every entry is nonpositive. If $r=w s_{i} w^{-1}$, then the image of $1-r$ is $w$ times the image of $1-s_{i}$, and thus $w \circ \iota_{i}$ identifies $E_{i}$ with $E_{r}$. Each $E_{r}$ is afforded with precisely two such identifications, of which we naturally choose the one corresponding to a positive root. We furthermore see that conjugate simple reflections give rise to equivalent systems of identifications of root curves.

Corollary 3.7. Relative to the action of $W$ on $\prod_{i} E_{i}$ arising in this way, any two reflections have distinct root curves.

Proof. Assuming without loss of generality that $W$ is irreducible, we find that each $E_{i}$ is isogenous to $E_{1}$ in an essentially canonical way (choose the isogeny of smallest degree among the "positive" isogenies), and then see that two root curves agree iff the corresponding maps $E_{1} \rightarrow \prod_{i} E_{i}$ correspond to proportional real vectors, making the two reflections agree.

More generally, if $B$ is an abelian variety with trivial action of $W$, we could consider the image $B \times E_{1} \times \cdots \times E_{n}$ under a $W$-equivariant isogeny. We will say that the abelian variety $A$ arising in this way has an action of $W$ of "root type". Note that as in the finite case, we may always arrange for the kernel of the isogeny to be not just preserved by $W$ but fixed elementwise by $W$, as otherwise there will be kernel elements contained in root curves. We will also need the dual notion: an abelian variety with an action of $W$ is of "coroot type" if its dual is of root type. These are equivalent for finite groups, or more generally for Coxeter groups with nondegenerate Cartan matrices, but in the affine case the two notions do not agree. Note that in the coroot type case, rather than having well-behaved positivity for roots, we have well-behaved positivity for coroots: for each conjugacy class of reflections, we can choose isomorphisms between the corresponding coroot curves and a fixed curve $E$ in such a way that the resulting set of maps to $E$, together with their negatives, are in equivariant, sign-preserving bijection with the corresponding set of root vectors. 
Consider the case of the affine Weyl group of type $\tilde{A}_{2}$. Since $m_{i j}=3, k_{i j}=1$, we see that each $\mu_{i j}$ is an isomorphism, and the positivity assumption forces the isomorphisms to be consistent. We thus obtain the following faithful action on $E^{3}$ :

$$
\begin{aligned}
& s_{0}\left(x_{0}, x_{1}, x_{2}\right)=\left(x_{1}+x_{2}-x_{0}, x_{1}, x_{2}\right), \\
& s_{1}\left(x_{0}, x_{1}, x_{2}\right)=\left(x_{0}, x_{0}+x_{2}-x_{1}, x_{2}\right), \\
& s_{2}\left(x_{0}, x_{1}, x_{2}\right)=\left(x_{0}, x_{1}, x_{0}+x_{1}-x_{2}\right) .
\end{aligned}
$$

This action fixes the diagonal copy of $E$, but does not fix any morphism to $E$. It follows that the corresponding action on the dual variety fixes a morphism to $E$, but does not fix any curve. In fact, we see that the dual action takes the form

$$
\begin{aligned}
& s_{0}\left(x_{0}, x_{1}, x_{2}\right)=\left(-x_{0}, x_{0}+x_{1}, x_{0}+x_{2}\right), \\
& s_{1}\left(x_{0}, x_{1}, x_{2}\right)=\left(x_{0}+x_{1},-x_{1}, x_{1}+x_{2}\right), \\
& s_{2}\left(x_{0}, x_{1}, x_{2}\right)=\left(x_{0}+x_{2}, x_{1}+x_{2},-x_{2}\right),
\end{aligned}
$$

from which we may see that the corresponding root curves do not even generate $E^{3}$.

For our purposes, we will in fact prefer actions of coroot type. The main issue with actions of root type in the affine case is that since there are only finitely many distinct coroots, the kernel of any given coroot map is fixed by infinitely many reflections. For instance, in the above $\tilde{A}_{2}$ example, both $s_{0}$ and $s_{1} s_{2} s_{1}$ fix the hypersurface $x_{1}+x_{2}=2 x_{0}$ pointwise. However, the dual of the standard model, though of coroot type, is badly behaved for other reasons: the product of root curves corresponding to the finite Weyl group does not inject, and the image of the product is fixed by the translation subgroup.

Suppose $\widetilde{W}=\left\langle s_{0}, \ldots, s_{n}\right\rangle$ is an affine Weyl group (with associated finite Weyl group $W=$ $\left.\left\langle s_{1}, \ldots, s_{n}\right\rangle\right)$, and that the abelian variety $A$ is equipped with an action of $\widetilde{W}$ of coroot type. The $\widetilde{W}$-invariant subvariety of $A^{\vee}$ has codimension $n$, and induces by duality a universal equivariant morphism $A \rightarrow B$ such that $\widetilde{W}$ acts trivially on $B$ and the fibers have dimension $n$. (In other words, we may interpret the original action as a family of actions on $n$-dimensional varieties.) In contrast, the invariant subvariety of $A$ has codimension $n+1$, and thus its image in $B$ has codimension 1 . Thus if we base change by a suitable isogeny $B^{\prime} \rightarrow B$, we may arrange for $B$ to be the product of $A^{\widetilde{W} 0}$ by an elliptic curve, allowing us to split off that factor and reduce to the case that $B$ is an elliptic curve $E$. Now, since $W$ is finite, $A^{W 0}$ has codimension $n$, and is thus itself an elliptic curve, which necessarily surjects onto $E$. Although this curve $A^{W 0}$ is not preserved by $\widetilde{W}$, we may still base change by it, and thus find that the natural action of $W$ on $A^{W 0} \times A_{W}$ extends to an action of $\widetilde{W}$ in such a way that the isogeny $A^{W 0} \times A_{W} \rightarrow A$ is equivariant. (Note, however, that the factorization itself is not equivariant; the projection to $A_{W}$ is not an equivariant map.)

We can describe this action explicitly on generators. Of course, for $1 \leq i \leq n, s_{i}(z, x)=$ $\left(z, s_{i}(x)\right)$, so only $s_{0}$ is nontrivial. The root curve associated to $s_{0}$ is the same as the root curve associated to the reflection $r$ in the relevant root of $W$, and the action on $0 \times A_{W}$ is the same as that of $r$. We thus see that $s_{0}(z, x)=(z, r(x)+\zeta(z))$ for some (nonzero) morphism $\zeta: A^{W 0} \rightarrow E_{r}$. Conversely, it is easy to see that any action of this form has coroot type.

We may view this action as a family of actions of $\widetilde{W}$ on $A_{W}$ parametrized by $z$, with the one caveat being that the action no longer preserves the identity; indeed, the translation subgroup of $\widetilde{W}$ acts (unsurprisingly) as translations of $A_{W}$. Of course, the action on a given fiber depends only on the point $q:=\zeta(z)$, and we easily see that it is faithful precisely when $q$ is non-torsion. (It follows from the above considerations that this is the typical form of an action of coroot type, up to base change and twisting by a $\left(A_{W}\right)^{W}$-torsor.)

Note that in this construction, we may as well start with a given action of $W$ and then adjoin $s_{0}$. In non-simply-laced cases, one must choose an orbit of roots and then obtain $s_{0}$ by 
shifting the action of the reflection in the highest root of that orbit by an element $q$ of the corresponding root curve. This produces an action of the affine Weyl group $W \ltimes \Lambda$, where $\Lambda$ is the free abelian group generated by the root maps in that orbit. It is worth noting that the choice of orbit is entirely orthogonal to the direction (if any) of the arrows in the finite Dynkin diagram: e.g., each of the three versions of the finite diagram $B_{n}=C_{n}$ gives rise to both an action of $\tilde{B}_{n}$ and an action of $\tilde{C}_{n}$, and similarly in the $G_{2}$ and $F_{4}$ cases, there are two natural extensions to actions of the corresponding affine groups. It is also worth noting that the various base changes required to put the action in this form can eliminate some of the information present in the original coroot model; in particular, in the $\tilde{C}_{n}$ case (including $\tilde{C}_{1}=\tilde{A}_{1}$ ), the special node is connected to the rest of the Dynkin diagram by an arrow, and thus there is a choice of isogeny in the coroot model. This includes some exotic coroot models in which $E_{0}$ and $E_{n}$ are merely 4 -isogenous and the corresponding family of abelian varieties with $\tilde{C}_{n}$ action has no section.

Returning to the finite case, suppose that $A / S$ is a family of abelian varieties (over an integral base $S$ ) equipped with a faithful action of the finite Weyl group $W$ by reflections, and suppose moreover that we are given a $W$-invariant ample line bundle $\mathcal{L}$ on $A$. This can be made equivariant by taking the action on the fiber at 1 to be trivial, and we may then ask when the map $s \mapsto \operatorname{dim} \Gamma\left(A_{s} ; \mathcal{L}\right)^{W}$ is constant on $S$. By the reductions of the previous section, this reduces to considering the corresponding question for $A_{W}$, which is very nearly a variety of the form we considered above. To be precise, the root curves in each irreducible component of $W$ are isogenous, and since indecomposable finite Weyl groups have at most two conjugacy classes of reflections, we see that each component is associated to a point of $\mathcal{X}_{0}, \mathcal{X}_{0}(2)$, or $\mathcal{X}_{0}(3)$. To ensure that we can apply our previous results, we must insist that the induced line bundles on the root curves be suitable; to wit, we insist that for each reflection, $\left.\mathcal{L}\right|_{E_{r}} \cong \mathcal{L}_{2 d_{r}, 0 ; E_{r}}$ for some positive integer $d_{r}$, clearly constant on conjugacy classes of involutions. We thus see that the only possible issues arise when (a) one of the curves $E_{r}$ is supersingular of characteristic dividing $W$, or (b) the "root kernel", i.e., the kernel of $\prod_{i} E_{i} \rightarrow A$, fails to be diagonalizable. In fact, the first condition turns out not to be necessary.

Lemma 3.8. Let $\mathcal{L}$ be a $W$-invariant ample line bundle on an abelian variety $A$ such that there are positive integers $d_{i}$ such that $\left.\mathcal{L}\right|_{E_{i}} \cong \mathcal{L}_{2 d_{i}, 0 ; E_{i}}$ for each $i$. Then for any section $f \in \Gamma(A ; \mathcal{L})$, the antisymmetrization $\sum_{w \in W} \sigma(w) w f$ vanishes along the divisor $\sum_{r \in R(W)}[\operatorname{ker}(r-1)]$.

Proof. We may write $\sum_{w \in W} \sigma(w) w=(1-r) \sum_{w \in W_{0}} w$, where $W_{0}$ is the even subgroup of $W$. Since the divisors $\operatorname{ker}(r-1)$ are transverse for distinct $r$, it thus suffices to show that $(1-r) f$ vanishes along the divisor $[\operatorname{ker}(r-1)]$. We thus reduce to the case that $W$ has rank 1 . In other words, $A$ is a quotient of a variety $B \times E$ (with $r$ acting trivially on $B$ ) obtained by identifying some subgroup $K \subset E[2]$ with a subgroup of $B$. The action of $r$ lifts to $B \times E$, and we conclude (by considering how $[-1]$ acts on sections of $\mathcal{L}_{2 d}$ ) that the antisymmetrization of any section of the pulled back line bundle must vanish on the divisor $B \times E[2]$. This is the preimage of the divisor $(B \times E[2]) / K$, which in turn is precisely the kernel of $r-1$ as required.

This gives us the following possible approach to controlling invariants in such bundles. Let $\mathcal{L}_{\Delta}$ be the line bundle $\mathcal{O}_{A}\left(\sum_{r \in R(W)}[\operatorname{ker}(r-1)]\right)$, but equipped with the equivariant structure which is trivial at the identity. If there is a section $g \in \Gamma\left(A_{W} ; \mathcal{L}_{\Delta}\right)$ with nontrivial antisymmetrization, then the operation $f \mapsto \frac{\sum_{w \in W} \sigma(w) w(g f)}{\sum_{w \in W} \sigma(w) w(g)}$ induces an idempotent on any $\Gamma(A ; \mathcal{L})$ which projects onto the symmetric subspace. More generally, if we have a family of such varieties such that such a section $g$ exists locally (or, equivalently, on every fiber), then we obtain such idempotents locally, and thus the spaces $\Gamma\left(A_{s} ; \mathcal{L}\right)^{W}$ are fibers of a vector bundle, implying that their dimensions are constant. 
Theorem 3.9. Suppose $A / S$ is a family of abelian varieties equipped with a faithful action by reflections of the finite Weyl group $W$, and let $\mathcal{L}$ be a $W$-invariant ample line bundle on $A$ such that the restriction to every root curve of every fiber is isomorphic to an even power of $\mathcal{L}_{1}$. If the root kernel of $A$ is diagonalizable, then the functions $s \mapsto \operatorname{dim} \Gamma\left(A_{s} ; \mathcal{L}\right)^{W}$ and $s \mapsto \operatorname{dim}\left(\left(\sum_{w \in W} \sigma(w) w\right) \Gamma\left(A_{s} ; \mathcal{L}\right)\right)$ are constant on $S$.

Proof. We first observe that by Lemma 2.35, the claims hold for $A$ iff they hold for $A_{W}$, a.k.a. the image of $\prod_{i} E_{i} \rightarrow A$. We may thus without loss of generality assume that the morphism $\prod_{i} E_{i} \rightarrow A$ is an isogeny such that $W$ acts trivially on the root kernel $K$. By assumption, $K$ is diagonalizable, so that $\Gamma\left(\prod_{i} E_{i} ; \mathcal{L}\right)$ decomposes into $K$-eigenspaces, and this decomposition is compatible with the action of $W$. It then follows by semicontinuity that the claims hold for $A$ if they hold for $\prod_{i} E_{i}$. Since this is a product over the components of $W$, we may assume without loss of generality that $W$ is indecomposable.

We may then reduce as discussed to showing that for any elliptic root datum corresponding to an indecomposable finite Weyl group, the corresponding line bundle $\mathcal{L}_{\Delta}$ contains a section with nontrivial antisymmetrization. (There is also the technical, but easy to verify, condition that $\left.\mathcal{L}_{\Delta}\right|_{E_{r}} \cong \mathcal{L}_{2 d_{r}, 0 ; E_{r}}$ for suitable positive integers.)

Here we may use the classification of finite Weyl groups. The simplest case is $W=A_{n}$, in which case we may identify $\prod_{i} E_{i}$ with the subvariety of $E^{n+1}$ on which $\sum_{i} z_{i}=0$. By induction in $n$ (with trivial base case $n=0$ ), the result holds for $n-1$, and thus any $S_{n}$-anti-invariant section can be obtained by antisymmetrization over $S_{n}$. It thus suffices to show that there is an $S_{n}$-anti-invariant section that when summed over coset representatives of $S_{n+1} / S_{n}$ with appropriate sign gives a nonzero result. Equivalently, by dividing by the appropriate product of $\vartheta$ functions, we need to find an $S_{n}$-invariant function with suitable poles that symmetrizes to a nonzero constant. For auxiliary parameters $y_{1}, \ldots, y_{n+2}$, we may consider the function

$$
\frac{\prod_{1 \leq i \leq n+2} \vartheta\left(z_{n+1}-y_{i}\right) \prod_{1 \leq i \leq n} \vartheta\left(Y-z_{i}\right)}{\prod_{1 \leq i \leq n} \vartheta\left(z_{n+1}-z_{i}\right)},
$$

where $Y=\sum_{1 \leq i \leq n+2} y_{i}$. Summing this over $S_{n+1} / S_{n}$ gives a function with no poles, which must therefore be constant; on the other hand, of the $n+1$ terms that result, all but one vanishes when $z_{n+1}=Y$. We thus find that

$$
\sum_{w \in S_{n+1} / S_{n}} w \cdot \frac{\prod_{1 \leq i \leq n+2} \vartheta\left(z_{n+1}-y_{i}\right) \prod_{1 \leq i \leq n} \vartheta\left(Y-z_{i}\right)}{\prod_{1 \leq i \leq n} \vartheta\left(z_{n+1}-z_{i}\right)}=\prod_{1 \leq i \leq n+2} \vartheta\left(Y-y_{i}\right),
$$

which is generically nonzero. (Note that the case $n=1$ is a version of the standard addition law for theta functions.) This identity is a disguised form of a classical theta function identity; see the discussion around [29, equation (1.22)].

For types $B / C / D$, we may similarly reduce to lower rank cases, noting that $D_{2}$ and $B_{1}$ both follow from the result for $A_{1}$. There are four cases to consider: the action of $D_{n}$ on $E \otimes \Lambda_{D_{n}}$, the action of $B_{n}$ on the same variety, the action of $C_{n}$ on $E \otimes \mathbb{Z}^{n}$ (following [18], we label the cases by the dual root system), and the action of $B C_{n}$ on the variety $E_{\Lambda_{D_{n}}, \mathbb{Z}^{n}}$ associated to a point of $\mathcal{X}_{0}(2)$ lying over $E$. In each case, there is a natural isogeny to $E \otimes \mathbb{Z}^{n}$, and it turns out we can choose the function being symmetrized to be the pullback of a function on $E \otimes \mathbb{Z}^{n}$. The simplest identity corresponds to the $C_{n}$ case, valid for all $n \geq 1$ :

$$
\begin{gathered}
\sum_{w \in C_{n} / C_{n-1}} w \cdot \frac{\prod_{1 \leq i \leq 2 n+1} \vartheta\left(z_{n}-y_{i}\right) \prod_{1 \leq i \leq n} \vartheta\left(Y+z_{i}\right) \prod_{1 \leq i<n} \vartheta\left(Y-z_{i}\right)}{\vartheta\left(2 z_{n}\right) \prod_{1 \leq i<n} \vartheta\left(z_{n}+z_{i}\right) \vartheta\left(z_{n}-z_{i}\right)} \\
=\prod_{1 \leq i \leq 2 n+1} \vartheta\left(Y-y_{i}\right),
\end{gathered}
$$


with $Y=\sum_{1 \leq i \leq 2 n+1} y_{i}$; if we expand this out as a sum of $2 n$ terms, we find that it is simply the special case $\left(z_{1}, \ldots, z_{2 n}\right) \mapsto\left(-z_{n}, \ldots,-z_{1}, z_{1}, \ldots, z_{n}\right)$ of the $S_{2 n} / S_{2 n-1}$ identity. In characteristic not 2, we may set $y_{2 n-2}, \ldots, y_{2 n+1}$ to be the four points of $E[2]$ to obtain an identity for $D_{n}$, $n>2$ :

$$
\begin{gathered}
\sum_{w \in D_{n} / D_{n-1}} w \cdot \frac{\prod_{1 \leq i \leq 2 n-3} \vartheta\left(z_{n}-y_{i}\right) \prod_{1 \leq i \leq n} \vartheta\left(Y+z_{i}\right) \prod_{1 \leq i<n} \vartheta\left(Y-z_{i}\right)}{\prod_{1 \leq i<n} \vartheta\left(z_{n}+z_{i}\right) \vartheta\left(z_{n}-z_{i}\right)} \\
=\vartheta(2 Y) \prod_{1 \leq i \leq 2 n-3} \vartheta\left(Y-y_{i}\right),
\end{gathered}
$$

where $Y=\sum_{1 \leq i<2 n-3} y_{i}$. Since this identity is expressed entirely in terms of $\vartheta$, it continues to hold in characteristic 2 . If we only specialize three parameters to the nonzero 2 -torsion points, we instead obtain (for $n \geq 2$ ):

$$
\begin{gathered}
\sum_{w \in B_{n} / B_{n-1}} w \cdot \frac{\prod_{1 \leq i \leq 2 n-2} \vartheta\left(z_{n}-y_{i}\right) \prod_{1 \leq i \leq n} \vartheta\left(Y+z_{i}\right) \prod_{1 \leq i<n} \vartheta\left(Y-z_{i}\right)}{\vartheta\left(z_{n}\right) \prod_{1 \leq i<n} \vartheta\left(z_{n}+z_{i}\right) \vartheta\left(z_{n}-z_{i}\right)} \\
=\frac{\vartheta(2 Y)}{\vartheta(Y)} \prod_{1 \leq i \leq 2 n-2} \vartheta\left(Y-y_{i}\right) .
\end{gathered}
$$

We omit the analogous identity for $B C_{n}$ (obtained from the $C_{n}$ identity by setting two of the $y_{i}$ to be the 2-torsion points not in the kernel of $\phi$ ) due to notational difficulties with using $\vartheta$ in the presence of isogenies, but note that for purposes of the reduction there is no reason we cannot simply use the $B_{n}$ identity.

For the seven exceptional cases (two each of $G_{2}$ and $F_{4}$ along with the simply laced cases $E_{6}$, $E_{7}, E_{8}$ ), we observe that the relevant line bundle comes from a polarization of degree a multiple of 6 , and we may thus use Lemma 2.34 to reduce to a smaller group. That is, we obtain an equivariant isomorphism

$$
\Gamma\left(A ; \mathcal{L}_{\Delta}\right) \cong \bigoplus_{H} \operatorname{Ind}_{H}^{W} \operatorname{Res}_{H}^{W} \Gamma\left(A^{\prime} ; \mathcal{L}_{\Delta}\right)
$$

where $H$ ranges over the point stabilizers in the different orbits of the action of $G$ on an appropriate diagonalizable 2- or 3-group. The image of antisymmetrization on the left is thus the direct sum of terms

$$
\left(\sum_{h \in H} \sigma(h) h\right) \operatorname{Res}_{H}^{W} \Gamma\left(A^{\prime} ; \mathcal{L}_{\Delta}\right),
$$

and since $H$ is a reflection group in each case, we may apply induction. (In fact, only that term which is nonzero in characteristic 0 has any hope of contributing).

For instance, for $E_{8}$, the variety is $E^{8}$ with polarization given by 30 times the Cartan matrix of $E_{8}$. In characteristic not 2, we may use the 2-part of $\Lambda_{E_{8}} / 30 \Lambda_{E_{8}}$ to split into eigenspaces, of which only one term survives. We thus reduce to showing that the descended line bundle on $E_{\Lambda_{E_{8}}, 2 \Lambda_{E_{8}}} \cong E_{2}^{8}$ (with nonproduct polarization) has a nontrivial antisymmetrization under the stabilizer $W\left(D_{8}\right)$. This is 2-isogenous to the standard $D_{8}$ model, and thus (since the characteristic is not 2) the image of antisymmetrization has the same dimension as in characteristic 0. A similar reduction using the 3-part reduces to antisymmetrization for $W\left(A_{8}\right)$ and proves the result for any characteristic other than 3 .

Remark 3.10. Results of $[18,31]$ in characteristic 0 actually compute the structure of the invariant ring (i.e., $\bigoplus_{d} \Gamma\left(E_{B, C} ; \mathcal{L}_{Q}^{d}\right)$, where $Q$ is the minimal invariant polarization satisfying 
the evenness requirement) and find that in each case the result is a free polynomial ring in generators of degrees that can be read off of the coefficients of the highest short (co)root. This suggests that something similar should hold in arbitrary characteristic. It would be natural in this context to also consider actions of complex reflection groups on varieties isogenous to $E^{n}$ where $j(E) \in\{0,1728\}$, or even quaternionic reflection groups in the case of supersingular curves of characteristic 2 or 3 .

Remark 3.11. The diagonalizability hypothesis is necessary, at least as far as the antisymmetrization claim is concerned. For example, suppose $x \in E[3]$ is a nontrivial 3-torsion point, and consider the quotient $A$ of the sum 0 subvariety of $E^{3}$ by the subgroup generated by $(x, x, x)$. The image in $A$ of the point $(0, x,-x)$ is negated by every reflection, and is thus not contained in any reflection hypersurface, but still has nontrivial stabilizer $\mathbb{Z} / 3 \mathbb{Z} \subset S_{3}$. It follows that in characteristic 3, the antisymmetrization of any section of an ample line bundle will vanish at this point, and thus for no ample line bundle is the dimension of the image of antisymmetrization the same as in characteristic 0 (except, of course, when there are no antisymmetric elements in characteristic 0). The invariants remain well-behaved, however, as the invariant ring is the same as that of $\langle x\rangle$ on $\mathbb{P}^{2}$; this is a permutation representation, so its Hilbert series is independent of the characteristic.

In the sequel, we will need some understanding of equivariant gerbes on abelian schemes, and thus in particular will want to understand $H^{1}(W ; \operatorname{Pic}(A))$. This turns out to be nearly trivial when $W$ is finite and the map $\prod_{i} E_{i} \rightarrow A$ is an isomorphism, in that any nontrivial cohomology class remains nontrivial when restricted to some simple reflection. (In particular, $H^{1}(W ; \operatorname{Pic}(A)$ ) is 2 -torsion.)

For inductive purposes, we will need to consider a slightly larger class of actions. Given an elliptic root datum of finite type and a point $u \in \prod_{i} E_{i}(S)$, we may define an action of $W$ on $\prod_{i} E_{i}$ by

$$
s_{i}\left(x_{1}, \ldots, x_{n}\right)=\left(x_{1}, \ldots, x_{i-1},-x_{i}+\sum_{j \neq i} \mu_{j i}\left(x_{j}\right)+u_{i}, x_{i+1}, \ldots, x_{n}\right) ;
$$

this is of course no longer a pointed action of $W$, but is instead the twist by a class in $H^{1}\left(S ; A^{W}\right)$ determined by $u$. (More precisely, the cocycle corresponding to $u$ is fppf locally a coboundary, and the nonuniqueness of this representation gives a class in $H^{1}\left(S ; A^{W}\right)$.) (And, of course, a class in $H^{1}\left(S ; A^{W}\right)$ is representable in this form iff the corresponding $A$-torsor has a section.)

Lemma 3.12. Let $W$ be a finite Weyl group of rank 2 , and let $X=E_{1} \times E_{2}$ be equipped with an action of the above form such that the root datum has $r_{1} \geq r_{2}$. Then the kernel of $\sum_{w \in W}(-1)^{\ell(w)} w$ on $\operatorname{Pic}(X)$ is spanned by $\pi_{1}^{*} \operatorname{Pic}\left(E_{1}\right)$ and $\operatorname{Pic}(X)^{\left\langle s_{1}\right\rangle}$.

Proof. It suffices to prove the corresponding claims for $\operatorname{Pic}^{0}(X)$ and $\mathrm{NS}(X)$ (modulo the requirement in the latter case that the classes lift to actual bundles still of the required form). Since $\operatorname{Pic}^{0}(X)$ is generated by $\pi_{1}^{*} \operatorname{Pic}^{0}\left(E_{1}\right)$ and $\pi_{2}^{*} \operatorname{Pic}^{0}\left(E_{2}\right)$, with the latter $s_{1}$-invariant, we find that the antisymmetrizer vanishes on $\operatorname{Pic}^{0}(X)$, and the claim is immediate. The Néron-Severi group may be identified with the space of matrices of the form

$$
Q=\left(\begin{array}{cc}
a & b \\
b^{\vee} & c
\end{array}\right)
$$

with antisymmetrization

$$
\left(\begin{array}{cc}
0 & 4 b \\
4 b^{\vee} & 0
\end{array}\right)
$$


when $m_{12}=2$ and

$$
\left(\begin{array}{cc}
0 & m_{12}\left(b \mu_{21}^{-1}-\left(b \mu_{21}^{-1}\right)^{\vee}\right) \mu_{21} \\
-m_{12} \mu_{21}^{\vee}\left(b \mu_{21}^{-1}-\left(b \mu_{21}^{-1}\right)^{\vee}\right) & 0
\end{array}\right),
$$

when $m_{12} \in\{3,4,6\}$. It follows in either case that the vanishing of the antisymmetrization implies $b \in \mathbb{Z} \mu_{21}$. (Here for $m_{12} \in\{3,4,6\}$, we use the fact that $\operatorname{deg}\left(\mu_{21}\right) \leq \operatorname{deg}\left(\mu_{12}\right)$ and $\mu_{12} \mu_{21} \in\{0,1,2,3\}$, so that $\operatorname{deg}\left(\mu_{21}\right)$ is squarefree and an isogeny is in $\mathbb{Q} \mu_{21}$ iff it is in $\mathbb{Z} \mu_{21}$.) In particular, the kernel of the antisymmetrizer is spanned by the matrices

$$
\left(\begin{array}{ll}
1 & 0 \\
0 & 0
\end{array}\right), \quad\left(\begin{array}{cc}
2 & -\mu_{21} \\
-\mu_{21}^{\vee} & \mu_{21}^{\vee} \mu_{21}
\end{array}\right), \quad\left(\begin{array}{ll}
0 & 0 \\
0 & 1
\end{array}\right) .
$$

The first matrix is in the pullback of $\pi_{1}^{*}$, while the second and third are $s_{1}$-invariant (and, in fact, images of $s_{1}$-invariant bundles).

Proposition 3.13. Let $X / S=\prod_{i} E_{i}$ be equipped with an action of the finite Weyl group $W$ of the above form, and let $z \in Z^{1}(W ; \operatorname{Pic}(X))$ be such that $z_{s_{i}}$ is a coboundary for every $i$. Then $z$ is a coboundary.

Proof. Since $W$ is finite, its diagram is a forest, and we may thus order the roots in such a way that $s_{1}$ corresponds to a leaf, and thus without loss of generality commutes with $s_{3}, \ldots, s_{n}$. Moreover, since every component has at most two values of $r_{i}$, we may arrange to have $r_{1} \geq r_{i}$ for all $i$. If we view $X$ as a family of abelian varieties over $E_{1}$, then we see that the action of $W_{\{2, \ldots, n\}}$ is still of the above form, and thus the corresponding restriction of $z$ is a coboundary by induction. We may thus reduce to the case that $z_{s_{2}}=\cdots=z_{s_{n}}=0$.

Now, consider the action of $\left\langle s_{1}, s_{2}\right\rangle$ on $X$ viewed as a family over $\prod_{3 \leq i \leq n} E_{i}$, and let $v \in$ $\operatorname{Pic}(X)$ be such that $z_{s_{1}}=v-{ }^{s_{1}} v$. The cocycle condition on $z_{s_{1}}$ implies that $\sum_{w \in\left\langle s_{1}, s_{2}\right\rangle}(-1)^{\ell(w) w} v$ $=0$, and thus the lemma implies that we may replace $v$ by the pullback of a class in $E_{1} \times$ $\prod_{3 \leq i \leq n} E_{i}$ without changing $z_{s_{1}}$. Since the cocycle is trivial on $W_{\{3, \ldots, n\}}$ and $s_{1}$ commutes with this subgroup, $z_{s_{1}}$ must be $W_{\{3, \ldots, n\}}$-invariant, and thus a computation in the Néron-Severi group shows that $v$ is in the subgroup generated by $\operatorname{Pic}\left(E_{1}\right)$ and $\operatorname{Pic}\left(\prod_{3 \leq i \leq n} E_{i}\right)$. Since elements of the latter have no effect on $z_{s_{1}}$, we see that we may take $v \in \pi_{1}^{*} \operatorname{Pic}\left(E_{1}\right)$. This is $W_{\{2, \ldots, n\}}$-invariant, and thus $z=\partial v$ as required.

Remark. Note that this can fail if we quotient by a subgroup of $A^{W}$. Indeed, if $A=E^{2}$ is given the standard action of $W\left(A_{2}\right)$, then the result fails for the induced action on $A^{\vee} \cong E^{2}$. The cocycles in which all bundles have degree 0 (and which are coboundaries in rank 1 ) are themselves classified by $A^{\vee}(S)$, while a coboundary must be in the image of $A(S)$ under the invariant polarization, and these are different unless $E(S)$ is 3-divisible. There is no difficulty with the polarization, however, so although the induction breaks down, it may still be the case that the claim holds on the Néron-Severi group, which would imply an fppf local version of the proposition.

\section{Elliptic analogues of affine Hecke algebras}

Before proceeding to the construction of Hecke algebras associated to general elliptic root data, it will be helpful to consider the finite case, a generalization of the construction of [11]. Note that although we work with a finite group, the resulting Hecke algebras are most naturally thought of as elliptic analogues of affine Hecke algebras, as they include multiplication operators in addition to reflection operators. (In particular, [11] constructs affine Hecke algebras as degenerations of a special case of the construction given below.) 
Although the approach in [11] via residue conditions can (mostly) be extended to the infinite case, there are two alternate approaches for which the generalization is more straightforward: one as a space of operators preserving appropriate holomorphy conditions, the other as the subalgebra of the algebra of operators generated by the rank 1 subalgebras. Since we will need to understand the rank 1 case to give the second construction, we begin with the first.

In our application to noncommutative rational varieties below, we will need to be able to attach an arbitrary finite set of parameters to the endpoint roots of the affine $C_{n}$ diagram; we will thus give a version of the general construction in which each conjugacy class of reflections can be given arbitrarily many parameters. This, of course, includes a case without any parameters at all, which we consider first.

This "master" Hecke algebra has a third description which does not generalize well to the infinite case, but is simplest of all to give (and extend to actions of arbitrary finite groups). Let $X$ be a regular integral scheme, and let $G$ be a finite group acting faithfully on $X$. Then we define the master Hecke algebra $\mathcal{H}_{G}(X)$ to be the sheaf of algebras on $X / G$ given by $\mathcal{H}_{G}(X):=$ $\mathcal{E} \operatorname{nd}\left(\pi_{*} \mathcal{O}_{X}\right)$, where $\pi: X \rightarrow X / G$ is the quotient map.

If $\pi$ is flat, then $\mathcal{H}_{G}(X)$ is the endomorphism ring of a vector bundle, so is in particular an Azumaya algebra on $X / G$, and the category of quasicoherent $\mathcal{H}_{G}(X)$-modules is equivalent to the category of quasicoherent sheaves on $X / G$. However, this condition holds only rarely; even in the case when $G$ is a finite Weyl group acting on an abelian variety, this morphism can easily fail to be flat. For instance, consider the case $G=G_{2}$ acting on the sum zero subvariety $X$ of $E^{3}$ (as permutations and global negation). In characteristic not 2, consider the point $\left(\tau_{1}, \tau_{2}, \tau_{1}+\tau_{2}\right) \in E^{3}$, where $\tau_{1}, \tau_{2}$ generate $E[2]$. This point has stabilizer $Z(G)$, and is isolated in the subvariety fixed by its stabilizer, and thus we see that its image in $X / G$ is a singular point (of type $A_{1}$ ), and that the quotient morphism fails to be flat in a neighborhood of that orbit.

There are two prominent cases in which we do have flatness, namely the action of $A_{n}$ on the sum 0 subvariety of $E^{n+1}$ and the action of $C_{n}$ on $E^{n}$; in each case, the quotient morphism is flat because it is a quasi-finite morphism between regular schemes (from $E^{n}$ to $\mathbb{P}^{n}$, to be precise).

In general, although $\mathcal{H}_{G}(X)$ may not be an Azumaya algebra, we at least know that it is torsion-free, and thus may be viewed as contained in its generic fiber $\operatorname{End}_{k(X / G)}(k(X))$. Since $k(X)$ is Galois over $k(X / G)$, the generic fiber has an alternate description as a twisted group algebra $k(X)[G]$, giving rise to the following description of $\mathcal{H}_{G}(X)$. Denote the natural action of $\operatorname{Aut}(X)$ on $k(X)$ by ${ }^{g} f:=\left(g^{-1}\right)^{*} f$; we will also use a similar notation for the actions on line bundles and divisors. It will also be convenient to define (for finite $G$ ) ${ }^{G} f=\pi_{G}^{*} f$, where $f \in k(X / G)$ and $\pi: X \rightarrow X / G$ is the natural quotient.

Proposition 4.1. The master Hecke algebra $\mathcal{H}_{G}(X)$ is the subsheaf of the twisted group algebra $k(X)[G]$ such that for any $G$-invariant open subset $U, \Gamma\left(U / G ; \mathcal{H}_{G}(X)\right)$ consists of the operators $\sum_{i} c_{i} g$ such that for any $G$-invariant open $V$ and $f \in \Gamma\left(V ; \mathcal{O}_{X}\right), \sum_{i} c_{i}^{g} f \in \Gamma\left(U \cap V ; \mathcal{O}_{X}\right)$.

Proof. Indeed, $\mathcal{H}_{G}(X)$ is the subsheaf of $\operatorname{End}_{k(X / G)}(k(X))$ which on $U$ consists of endomorphisms preserving $\left.\mathcal{O}_{X}\right|_{U}$, or equivalently preserving global sections of $\left.\mathcal{O}_{X}\right|_{V}$ for all invariant $V \subset U$. The claim then follows by using the twisted group algebra description of the endomorphism ring and observing that $\Gamma\left(V ; \mathcal{O}_{X}\right) \subset \Gamma\left(U \cap V ; \mathcal{O}_{X}\right)$.

One consequence is that if $H \subset G$, then there is a natural inclusion $\mathcal{H}_{H}(X) \subset \mathcal{H}_{G}(X)$ (where we conflate $\mathcal{H}_{H}(X)$ with its direct image under $X / H \rightarrow X / G$ ). In addition, if $\alpha$ is an automorphism of $X$ that normalizes $G$, then there is a corresponding automorphism of $\mathcal{H}_{G}(X)$, either by pulling back through the induced automorphism of $X / G$, or on operators as $\sum_{g} c_{g} g \mapsto \sum_{g}{ }^{\alpha} c_{\alpha^{-1} g \alpha} g$.

Note that $\mathcal{H}_{G}(X)$ clearly contains a copy of the structure sheaf $\mathcal{O}_{X}$ as well as the operators $g$ for each $g \in G$, and thus contains a copy of the twisted group algebra $\mathcal{O}_{X}[G]$. It can, however, 
be bigger than the twisted group algebra. Consider the case of $G=\mu_{n}=\langle s\rangle$ acting on $X=\mathbb{A}^{1}$ in characteristic prime to $n$ by rescaling the variable $x$. Applying the operator $1+\zeta_{n} s+\zeta_{n}^{2} s^{2}+$ $\cdots+\zeta_{n}^{n-1} s^{n-1}$ to any function which is holomorphic at the origin gives a function which vanishes to order $n-1$ at the origin, and thus $\mathcal{H}_{\mu_{n}}\left(\mathbb{A}^{1}\right)$ contains the operator $x^{1-n}\left(1+\zeta_{n} s+\zeta_{n}^{2} s^{2}+\cdots+\right.$ $\zeta_{n}^{n-1} s^{n-1}$ ) not contained in $\mathcal{O}_{X}[G]$.

It turns out that this is the typical case in which the coefficients may have poles; more precisely, the only poles are associated to "complex reflections" in $G$ (relative to the action on $X$ ). It will be useful to consider a more general setting. We begin with a couple of local results.

Lemma 4.2. Suppose $R, S$ are discrete valuation rings, and suppose $\psi_{1}, \ldots, \psi_{n}: S \rightarrow R$ are distinct finite homomorphisms. Then the module of operators $\sum_{i} c_{i} \psi_{i}$ mapping $S$ to $R$ is a free $R$-module of rank $n$.

Proof. Consider the subalgebra $R \vec{\psi} S \subset R^{n}$ generated by the image of $S$ under $\left(\psi_{1}, \ldots, \psi_{n}\right)$. The monoid characters $\psi_{i}: S \backslash\{0\} \rightarrow R^{*}$ are linearly independent over $K_{R}$ and thus over $R$, so that $R \vec{\psi} S$ is free of rank $n$ as an $R$-module. An operator $\sum_{i} c_{i} \psi_{i}$ maps $S$ to $R$ iff the $K_{R^{-}}$ linear functional $\vec{c}$ maps $R \vec{\psi} S$ to $R$, and thus the space of such operators is isomorphic to the dual $R^{n}$.

Remark. It follows that the quotient by the submodule in which the coefficients are in $R$ has finite length, equal to the colength of $R \vec{\psi} S$ as a submodule of $R^{n}$.

For $n=1, R \vec{\psi} S=R$, and thus $(R \vec{\psi} S)^{*}=R$. We can also give an explicit description for $n=2$.

Corollary 4.3. Let $\psi_{1}, \psi_{2}: S \rightarrow R$ be finite homomorphisms of discrete valuation rings inducing the same action on residue fields. Then the operator $c_{1} \psi_{1}+c_{2} \psi_{2}: k(S) \rightarrow k(R)$ maps $S$ to $R$ iff $c_{1}, c_{2} \in \operatorname{det}(R \vec{\psi} S)^{-1}$ and $c_{1}+c_{2} \in R$.

Proof. The module $R \vec{\psi} S$ is spanned by elements of the form $\left(\psi_{1}(h), \psi_{2}(h)\right)$, or equivalently by the element $(1,1)$ and elements of the form $\left(0, \psi_{2}(h)-\psi_{1}(h)\right)$, and thus can be expressed as $R(1,1)+\operatorname{det}(R \vec{\psi} S)(0,1)$. The corresponding condition on the operator is that $c_{1}+c_{2} \in R$ and $c_{2} \in \operatorname{det}(R \vec{\psi} S)^{-1}$. These conditions imply that $c_{1} \in \operatorname{det}(R \vec{\psi} S)^{-1}$ as well.

Remark 4.4. For any $f \in S^{*}$, since $c_{1} \psi_{1}(f)+c_{2} \psi_{2}(f)-\psi_{1}(f)\left(c_{1}+c_{2}\right)=\left(\psi_{2}(f)-\psi_{1}(f)\right) c_{2}$ and $\psi_{2}(f)-\psi_{1}(f) \in \operatorname{det}(R \vec{\psi})$ we see that when $c_{2} \in \operatorname{det}(R \vec{\psi})^{-1}$, the conditions $c_{1}+c_{2} \in R$ and $c_{1} \psi_{1}(f)+c_{2} \psi_{2}(f) \in R$ are equivalent. This is useful in global situations in which one has twisted by a line bundle.

Remark 4.5. It follows that $\left(R\left(\psi_{1}, \psi_{2}\right) S\right)^{*}=R \psi_{1}+\operatorname{det}(R \vec{\psi} S)^{-1}\left(\psi_{1}-\psi_{2}\right)$.

For $n>2$, it is difficult to give an explicit description in general, but in the case of Coxeter groups, we can generally reduce to the $n=2$ case, using the following result. Given a homomorphism $\psi: S \rightarrow R$ of local rings, let $\bar{\psi}: k_{S} \rightarrow k_{R}$ denote the corresponding homomorphism of residue fields. Also, note that for any subset $I \subset\{1, \ldots, n\}$, there is a natural morphism $R \vec{\psi} S \rightarrow R\left(\psi_{i}: i \in I\right) S$.

Lemma 4.6. Suppose $R, S$ are discrete valuation rings, and suppose $\psi_{1}, \ldots, \psi_{n}: S \rightarrow R$ are distinct finite homomorphisms. Then the algebra $R \vec{\psi} S$ splits as the direct sum $\bigoplus_{\sigma} R\left(\psi_{i}: \bar{\psi}_{i}=\sigma\right) S$ of local rings, where $\sigma$ ranges over all morphisms $k_{S} \rightarrow k_{R}$. 
Proof. The radical of $R \vec{\psi} S$ is equal to its intersection with $\mathfrak{m}_{R}^{n}$, and the quotient is a product of copies of $k_{R}$, with one for each distinct reduction $\bar{\psi}_{i}$ (in particular, the radical is maximal and $R \vec{\psi} S$ local iff there is only one such reduction). If $R$ is complete, then we can lift the idempotents from the reduction to obtain the desired splitting. Moreover, the lifts are unique, and thus agree with the lifts one would have obtained if working inside the larger algebra $R^{n}$, i.e., the projections onto the given sets of coordinates. It thus follows that even if $R$ is not complete, the lifts in the completion of $R \vec{\psi} S$ agree with the projections, and thus said projections lie in $R \vec{\psi} S$.

Remark. By duality, the same splitting applies to the module of operators taking $S$ to $R$.

This leads to the following global result.

Lemma 4.7. Let $X, Y$ be normal integral schemes and let $\phi_{1}, \ldots, \phi_{n}: X \rightarrow Y$ be a collection of distinct finite morphisms. Let $\mathcal{M}_{\vec{\phi}}$ be the subsheaf of $k(X)^{n}$ which on an open subset $U \subset X$ consists of those $n$-tuples $\left(c_{1}, \ldots, c_{n}\right) \in k(X)^{n}$ such that for any open subset $V \subset Y$ and any function $f \in k(Y)$ holomorphic on $V$, the function $\sum_{i} c_{i} \phi_{i}^{*} f$ is holomorphic on $U \cap \bigcap_{i} \phi_{i}^{-1} V$. Then there is an n-tuple of divisors $\Delta_{i} \in \operatorname{Div}(X)$ such that $\mathcal{M}_{\vec{\phi}} \subset \bigoplus_{i} \mathcal{O}_{X}\left(\Delta_{i}\right)$, and each $\Delta_{i}$ is supported on those hypersurfaces on which $\phi_{j}=\phi_{i}$ for some $j \neq i$.

Proof. Let $D \subset X$ be a reduced irreducible hypersurface; we need to understand the possible singularities of the coefficients along $D$. Note that if $U_{1}, U_{2}$ are two open subsets meeting $D$, then $U_{1} \cap U_{2}$ also meets $D$, and thus any bound on singularities holding on $U_{1} \cap U_{2}$ also holds for global sections along $U_{1}, U_{2}$. We may thus take a limit along those open subsets meeting $D$. Similarly, since we are only considering holomorphy along $D$, we may take a limit over those $V$ meeting $\phi_{i}(D)$ for every $i$. In other words, the condition for $\left(c_{1}, \ldots, c_{n}\right)$ to be a section of the base change of $\mathcal{M}_{\vec{\phi}}$ to the local ring of $k(D)$ is that for any function $f$ which is holomorphic along $\phi_{1}(D), \ldots, \phi_{n}(D)$, the image $\sum_{i} c_{i} \phi_{i}^{*} f$ is holomorphic along $D$.

Now, given such an $n$-tuple, let $d$ be the maximum order of pole of a coefficient $c_{i}$ along $D$. The condition that $\sum_{i} c_{i} \phi_{i}^{*} f$ be holomorphic only depends on the value of $f$ modulo the intersections of the $d$-th powers of the maximal ideals at the divisors $\phi_{i}(D)$, and by the Chinese remainder theorem, the reductions corresponding to distinct divisors may be chosen independently. Taking those reductions to be 0 on all but $\phi_{j}(D)$ tells us that if the operator $\sum_{i} c_{i} \phi_{i}^{*}$ preserves holomorphy, then so does $\sum_{i: \phi_{i}(D)=\phi_{j}(D)} c_{i} \phi_{i}^{*}$.

We may as well assume, therefore, that the divisors $\phi_{i}(D)$ are all equal to the same divisor $D^{\prime}$ in $Y$, and thus reduce to the local case.

Remark. By mild abuse of notation, if $g_{1}, \ldots, g_{n} \in \operatorname{Aut}(X)$, then $\mathcal{M}_{\vec{g}}$ will denote the sheaf corresponding to the $n$-tuple $\vec{\phi}=\left(g_{1}^{-1}, \ldots, g_{n}^{-1}\right)$; i.e., the sheaf of operators $\sum_{i} c_{i} g_{i}$ that preserve holomorphy. This generalizes to the case of operators $\sum_{i} c_{i} g_{i} G: k(X / G) \rightarrow k(X)$ for a finite subgroup $G \subset \operatorname{Aut}(X)$, which act as $f \mapsto \sum_{i} c_{i}{ }^{g_{i} G} f$.

The local result for $n=2$ leads to the following result in the global setting. Given two finite morphisms $\phi, \psi$ of normal integral schemes, let $[\phi=\psi]$ denote the Cartier divisor obtained by removing all codimension $>1$ components from the subscheme on which $\phi$ and $\psi$ agree.

Corollary 4.8. With notation as above, suppose that for each $i$, the $n-1$ divisors $\left[\phi_{i}=\phi_{j}\right]$ are mutually transverse. Then $\mathcal{M}_{\vec{\phi}}$ may be identified with the subsheaf of

$$
\bigoplus_{i} \mathcal{O}_{X}\left(\sum_{j \neq i}\left[\phi_{j}=\phi_{i}\right]\right)
$$

in which the local sections $\left(c_{1}, \ldots, c_{n}\right)$ satisfy the additional "residue" conditions that $c_{i}+c_{j}$ is holomorphic along $\left[\phi_{i}=\phi_{j}\right]$ for all $i \neq j$. 
Proof. The condition on the divisors ensures that when we perform the various reductions, we will end up with local problems involving at most 2 morphisms. The case with one morphism is trivial (the coefficient is forced to be holomorphic, and this makes the operator preserve holomorphy), and the case with two is just Corollary 4.3 above.

Remark. If $\left[\phi_{i}=\phi_{j}\right]$ is reduced, then the residue conditions can of course be stated in terms of the residues of $c_{i}$ and $c_{j}$ with respect to any differential holomorphic and nonvanishing along $\left[\phi_{i}=\phi_{j}\right]$.

Note that we can also right-multiply operators by local sections of $\mathcal{O}_{Y}$, and thus obtain an $\left(\mathcal{O}_{X}, \mathcal{O}_{Y}\right)$-bimodule structure on $\mathcal{M}_{\vec{\phi}}$. Such a structure is equivalent to an $\mathcal{O}_{X} \otimes \mathcal{O}_{Y}$-module structure, or equivalently an $\mathcal{O}_{X \times Y}$-module structure. We will consider this structure in more detail when discussing the infinite case, but for the moment we observe the following.

Corollary 4.9. The induced sheaf $\mathcal{M}_{\vec{\phi}}$ on $X \times Y$ is a coherent subsheaf of $\bigoplus_{i}\left(1, \phi_{i}\right)_{*} \mathcal{O}_{X}\left(\Delta_{i}\right)$.

Remark. It is worth noting that this sheaf depends only on the set of morphisms $\left\{\phi_{1}, \ldots, \phi_{n}\right\}$, and not on the ordering, and the map from global sections to operators can be reconstructed from the bimodule structure. As a result, when considering sheaves on $X \times Y$, we allow the subscript to be a set rather than a sequence.

Corollary 4.10. The algebra $\mathcal{H}_{G}(X)$, viewed as an $\mathcal{O}_{X \times X}$-module, is coherent, and contained in the sum $\bigoplus_{g \in G}(1, g)_{*} \mathcal{O}_{X}(\Delta)$, where $\Delta$ is an effective divisor supported on the "reflection hypersurfaces" of $G$ on $X$ : the irreducible hypersurfaces which are fixed pointwise by some $g \in G$.

In the simplest elliptic case $A_{1}$ acting on $E$, the divisor $\Delta$ is precisely the divisor corresponding to the subscheme $E[2]$. In characteristic not 2, this subscheme is reduced, and thus the coefficients have at most simple poles at the 2 -torsion points, but in characteristic 2 , the coefficients can have double poles at the 2-torsion points of an ordinary curve, and a quadruple pole at the origin of a supersingular curve. This, of course, is an artifact of wild ramification; without that, a "reflection" of order $n$ will admit poles of order at most $n-1$ along the corresponding reflection hypersurfaces.

There is an important variation arising from the interpretation as an $\mathcal{O}_{X \times X}$-module: simply consider the sheaf $\mathcal{H}_{G ; \mathcal{L}_{1}, \mathcal{L}_{2}}(X):=\mathcal{H}_{G}(X) \otimes_{X \times X} \mathcal{L}_{2} \otimes \mathcal{L}_{1}^{-1}$ for invertible sheaves $\mathcal{L}_{2}, \mathcal{L}_{1}$ on $X$. Since both left- and right-multiplication by sections of $\mathcal{O}_{X / G}$ agree in $\mathcal{H}_{G}(X)$, this twisted version still descends to a sheaf on $X / G$, and the result moreover has induced compositions

$$
\mathcal{H}_{G ; \mathcal{L}_{1}, \mathcal{L}_{2}}(X) \otimes_{X / G} \mathcal{H}_{G ; \mathcal{L}_{2}, \mathcal{L}_{3}}(X) \rightarrow \mathcal{H}_{G ; \mathcal{L}_{1}, \mathcal{L}_{3}}(X) .
$$

In fact, as a sheaf on $X / G$, we have $\mathcal{H}_{G ; \mathcal{L}_{1}, \mathcal{L}_{2}}(X) \cong \mathcal{H} \mathrm{om}_{G / X}\left(\pi_{*} \mathcal{L}_{1}, \pi_{*} \mathcal{L}_{2}\right)$, with the obvious induced composition; this is most easily seen by representing $\mathcal{L}_{1}, \mathcal{L}_{2}$ by Cartier divisors, and observing that this turns $\mathcal{H}_{G ; \mathcal{L}_{1}, \mathcal{L}_{2}}(X)$ into the subsheaf of the meromorphic twisted group algebra taking the subsheaf of $k(X)$ corresponding to $\mathcal{L}_{1}$ into the subsheaf corresponding to $\mathcal{L}_{2}$. When $\mathcal{L}_{1}=\mathcal{L}_{2}$, we omit the second copy of $\mathcal{L}_{2}$, and note that the result is again a sheaf of algebras, with each $\mathcal{H}_{G ; \mathcal{L}_{1}, \mathcal{L}_{2}}(X)$ a bimodule that induces a Morita equivalence between $\mathcal{H}_{G ; \mathcal{L}_{1}}(X)$ and $\mathcal{H}_{G ; \mathcal{L}_{2}}(X)$.

In addition to the isomorphisms of such sheaves arising from isomorphisms of $\mathcal{L}_{1}, \mathcal{L}_{2}$, there are also isomorphisms coming from twisting both sheaves by a suitable invertible sheaf. Let $\mathcal{L}$ be a $G$-equivariant invertible sheaf on $X$ which "descends in codimension 1"; that is, there is an open subscheme of $X / G$ containing every codimension 1 point over which $\mathcal{L}$ descends to a line bundle. (Note that this is a local condition and is automatically satisfied on the complement of the reflection hypersurfaces.) Then for each reflection hypersurface $H$ with inertia group 
(i.e., pointwise stabilizer) $I_{H}$, there is a $I_{H}$-invariant neighborhood $U_{H}$ of the generic point of $H$ such that the $H$-equivariant sheaf $\mathcal{L}_{I_{H}}$ is equivariantly isomorphic to $\mathcal{O}_{U_{H}}$. It follows that there is a natural isomorphism $\mathcal{H}_{G ; \mathcal{L}}(X) \cong \mathcal{H}_{G}(X)$. Indeed, since $\mathcal{L}$ is $G$-equivariant, both algebras are naturally contained in $k(X)[G]$, and the conditions for any given reflection hypersurface are the same on both sides. This condition is automatically satisfied by the pullback of an invertible sheaf on $X / G$, but this is not necessary. For instance, in the case of $W\left(G_{2}\right)$ acting on the sum 0 subvariety of $E^{3}$, the quotient is a weighted projective space with generators of degree $1,1,2$. The pullback of $\mathcal{O}_{\mathbb{P}^{2}}(1)$ (from $X / W\left(A_{2}\right) \cong \mathbb{P}^{2}$ ) does not descend to a line bundle on $X / W\left(G_{2}\right)$ (since $\mathcal{O}_{X / W\left(G_{2}\right)}(1)$ is not invertible on such a weighted projective space), but does so if we remove the singular point of $X / W\left(G_{2}\right)$ and the three points of $X$ lying over it.

A particularly important instance of twisting by line bundles arises when we consider the natural involution on operators: $\sum_{g} c_{g} g \mapsto \sum_{g} g^{-1} c_{g}$.

Proposition 4.11. This gives a contravariant isomorphism $\mathcal{H}_{G}(X)^{\mathrm{op}} \cong \mathcal{H}_{G ; \omega_{X}}(X)$.

Proof. We need to show that $\sum_{g} c_{g} g$ preserves holomorphic functions iff $\sum_{g} g^{-1} c_{g}$ preserves holomorphic $n$-forms. By Lemma 4.7, it suffices to prove that the conditions on individual hypersurfaces are the same, and thus we may fix a hypersurface $D$ and restrict our attention to the case that every term in the sum gives the same divisor $g^{-1} D=D^{\prime}$. By duality, a function $f$ is holomorphic along $D$ iff $\operatorname{Res}_{D} f \omega=0$ for all $n$-forms $\omega$ which are holomorphic along $D$, and we may similarly detect holomorphy of $n$-forms by taking residues against test functions. In particular, for any function $f$ holomorphic along $D$, and any $n$-form $\omega$ holomorphic along $D^{\prime}$, we have

$$
\operatorname{Res}_{D^{\prime}}\left(\sum_{g} c_{g}^{g} f\right) \omega=\sum_{g} \operatorname{Res}_{D^{\prime}} c_{g}^{g} f \omega=\sum_{g} \operatorname{Res}_{D}{ }^{-1} c_{g} f^{g^{-1}} \omega=\operatorname{Res}_{D} f \cdot\left(\sum_{g} g^{-1}\left(c_{g} \omega\right)\right) .
$$

It follows that $\sum_{g} c_{g}{ }^{g} f$ is holomorphic along $D^{\prime}$ for all $f$ iff $\sum_{g} g^{-1}\left(c_{g} \omega\right)$ is holomorphic along $D$ for all $\omega$.

Let us now turn to the "elliptic" case, in which $G$ is a finite Weyl group $W$ acting by reflections on an abelian torsor $X / S$ over a normal integral base $S$. That is, the flat family $X / S$ is a torsor over an abelian variety $A / S$, and $W$ acts on $X$ in such a way that the induced action on $A$ is an action by reflections. Note that since the subvariety $X^{W}$ is the intersection of the simple reflection hypersurfaces, it has codimension at most $n$, so is nonempty (the corresponding intersection is nonempty and transverse in $A$, and thus the corresponding intersection number is positive) and thus a torsor over $A^{W}$; conversely, any $A^{W}$-torsor induces a corresponding family $X / S$ by twisting. The action of $W$ is faithful on every fiber, and this remains true if we view $X$ as a family over the quotient $S^{\prime}:=X / A_{W}$. We will see that the fibers of $\mathcal{H}_{W}(X)$ over $S$ are identified with the master Hecke algebras of the fibers, in a fairly strong way.

Given a reflection $r$, let $\left[X^{r}\right]$ denote the effective Cartier divisor cut out by the equation $r x=x$. Also, let $C_{r}$ denote the quotient of $X$ by the abelian subvariety $(r+1) A$; this, of course, is a torsor over the corresponding coroot curve $E_{r}^{\prime}$. The morphism $(r-1): X \rightarrow A$ factors through $C_{r}$ and has image $E_{r}$, so that there is a morphism $C_{r} \rightarrow E_{r}$ compatible with the isogeny $E_{r}^{\prime} \rightarrow E_{r}$, and thus $C_{r}$ corresponds to a class in $H^{1}\left(S ; \operatorname{ker}\left(E_{r}^{\prime} \rightarrow E_{r}\right)\right)$. Note that in contrast to the coroot curve, we cannot expect to have a natural torsor over the root curve inside $X$.

For the rank 1 case, we have the following immediate consequence of Lemma 4.7. Here a "hyperelliptic curve of genus 1" is a smooth genus 1 curve $C$ with a marked involution such that the quotient is (geometrically) rational; note that the torsor arising in the rank 1 case is always a family of such curves-with-involutions. 
Lemma 4.12. Let $C / S$ be a flat family of hyperelliptic curves of genus 1 with $G=A_{1}=\langle s\rangle$ acting by the marked involution. Then for any $G$-invariant open set $U, \Gamma\left(U ; \mathcal{H}_{A_{1}}(C)\right)$ consists of operators $f_{0}+f_{1}(s-1)$ such that $f_{0} \in \Gamma\left(U ; \mathcal{O}_{C}\right)$ and $f_{1} \in \Gamma\left(U ; \mathcal{O}_{C}\left(\left[C^{s}\right]\right)\right)$.

Remark 4.13. Note that

$$
f_{0}+f_{1}(s-1)=\left(f_{0}-f_{1}-{ }^{s} f_{1}\right)+(1+s)^{s} f_{1},
$$

and thus (since $f_{1}+{ }^{s} f_{1} \in \Gamma\left(U ; \mathcal{O}_{C}\right)$ ) we may also describe $\Gamma\left(U ; \mathcal{H}_{A_{1}}(C)\right)$ as the space of operators $f_{0}^{\prime}+(1+s) f_{1}^{\prime}$ such that $f_{0}^{\prime} \in \Gamma\left(U ; \mathcal{O}_{C}\right)$ and $f_{1}^{\prime} \in \Gamma\left(U ; \mathcal{O}_{C}\left(\left[C^{s}\right]\right)\right)$. This also follows from the above description of the adjoint once we realize that $\omega_{C}$ is the trivial line bundle with equivariant structure such that $s$ acts as -1 .

Remark 4.14. It will be useful in the sequel to know when an $A_{1}$-equivariant line bundle on $C$ descends in codimension 1 . If $\mathcal{L}$ is equivariantly isomorphic to $\mathcal{O}_{C}(D)$ for some symmetric Cartier divisor, then we may write $D$ as a linear combination of divisors $D^{\prime}$ and $D^{\prime \prime}+{ }^{s} D^{\prime \prime}$ with $D^{\prime}, D^{\prime \prime}$ irreducible and $D^{\prime}={ }^{s} D^{\prime}$. The latter case is the pullback of the image of $D^{\prime \prime}$ in $C / A_{1}$, and thus certainly has no effect on twisting, while if $D^{\prime}$ is not a component of $\left[C^{s}\right]$, then its image in $C / A_{1}$ is twice a divisor, so that again $D^{\prime}$ is a pullback. We are thus left to consider the linear combinations of reflection hypersurfaces, and thus determine that the condition on $D$ is precisely that the valuations along reflection hypersurfaces must be even (or no condition at all in characteristic 2 if the reflection hypersurface is inseparable over $S$ ). This, of course, is for the standard equivariant structure on $\mathcal{O}_{C}(D)$; in odd characteristic, we may twist by the sign character of $A_{1}$, in which case the condition becomes that the valuations along reflection hypersurfaces are odd. Note that in any event, a line bundle that descends in codimension 1 will restrict on the generic fiber to a power of the hyperelliptic bundle.

For rank $n$, we have the following.

Lemma 4.15. The $\mathcal{O}_{X / W}$-algebra $\mathcal{H}_{W}(X)$ is generated by the $\mathcal{O}_{X / W}$-subalgebras $\mathcal{H}_{\left\langle s_{i}\right\rangle}(X)$ for $1 \leq i \leq n$.

Proof. It follows from Lemma 4.7 that $\mathcal{H}_{W}(X)$ is generated as a left $\mathcal{O}_{X}$-module by the twisted group algebra $\mathcal{O}_{X}[W]$ along with the subsheaves arising from operators of the form $c_{w} w+c_{r w} r w$ for some reflection $r \in R(W)$. Now, by the above explicit description, each subalgebra $\mathcal{H}_{\left\langle s_{i}\right\rangle}(X)$ contains $\mathcal{O}_{X}\left[s_{i}\right]$, and thus the algebra they generate contains $\mathcal{O}_{X}[W]$. But then if we express $r$ above as $w_{1}^{-1} s_{i} w_{1}$ for some simple reflection $s_{i}$, we have

$$
c_{w} w+c_{r w} r w=c_{w} w+c_{w_{1}^{-1} s_{i} w_{1} w} w_{1}^{-1} s_{i} w_{1} w=w_{1}^{-1}\left({ }^{w_{1}} c_{w}+{ }^{w_{1}} c_{w_{1}^{-1} s_{i} w_{1} w} s_{i}\right) w_{1} w
$$

and find that $c_{w} w+c_{r w} r w$ is a local section of $\mathcal{H}_{W}(X)$ iff

$$
{ }^{w_{1}} c_{w}+{ }^{w_{1}} c_{w w_{1} s_{i} w_{1}^{-1}} s_{i}
$$

is a local section of $\mathcal{H}_{W}(X)$, iff it is a local section of $\mathcal{H}_{\left\langle s_{i}\right\rangle}(X)$. The claim follows.

Remark. Of course, the same argument shows that if $G$ is generated by a collection of cyclic groups meeting every conjugacy class of (generalized) reflections, then $\mathcal{H}_{G}(X)$ is generated by the corresponding subalgebras.

We can actually say a great deal more in the case of interest; not only is $\mathcal{H}_{W}(X)$ a flat sheaf in general, but we can in fact express it as an extension of invertible sheaves on $X$. The key ingredient is the fact that there is a natural partial order on $W$ (the Bruhat order), the weakest partial order such that if $w^{\prime} w^{-1}$ is a reflection, then $w$ and $w^{\prime}$ are comparable and ordered 
according to their length. Note that omitting any set of reflections from a reduced word for $w$ gives an element $w^{\prime} \leq w$, and classical results on Coxeter groups give the converse: for any reduced word for $w, w^{\prime} \leq w$ iff some word for $w^{\prime}$ (iff some reduced word for $w^{\prime}$ ) can be obtained by omitting reflections from the chosen reduced word.

Given an order ideal $I$ with respect to Bruhat order (i.e., a subset $I \subset W$ such that if $w \in I$ and $w^{\prime} \leq w$, then $w^{\prime} \in I$ ), we may consider the subsheaf $\mathcal{H}_{W}(X)[I]$ of $\mathcal{H}_{W}(X)$ consisting of those operators in which the coefficient of $w$ is 0 for $w \notin I$. Any chain of order ideals induces in this way a filtration, and we will show that in the case of a maximal chain, the subquotients of the filtration are invertible sheaves on $X$. Let $[\leq w]$ denote the order ideal consisting of elements $\leq w$

For any element $w \in W$, define a divisor $D_{w}:=\sum_{r \in R(W), r w<w}\left[X^{r}\right]$.

Lemma 4.16. Let $I$ be a Bruhat order ideal, and suppose that $w$ is a maximal element of $I$. Then there is a short exact sequence

$$
0 \rightarrow \mathcal{H}_{W}(X)[I \backslash\{w\}] \subset \mathcal{H}_{W}(X)[I] \rightarrow \mathcal{O}_{X}\left(D_{w}\right) \rightarrow 0 .
$$

Proof. By definition, $\mathcal{H}_{W}(X)[I \backslash\{w\}]$ is the kernel of the "coefficient of $w$ " map on $\mathcal{H}_{W}(X)[I]$, so we first need to show that the coefficient of $w$ is contained in $\mathcal{O}_{X}\left(D_{w}\right)$. It follows from Corollary 4.8 that the coefficient has polar divisor bounded by $\sum_{w^{\prime} \in(I \backslash\{w\}) \cap R(W) w}\left[X^{w^{\prime} w^{-1}}\right]$. If $w^{\prime} w^{-1}$ is a reflection, then $w^{\prime}$ is comparable to $w$, which since $w$ is maximal in $I$ implies that $w^{\prime}<w$, and thus the bound on the divisor is $D_{w}$ as required.

It remains only to show that the map is surjective. Choose a reduced word $w=s_{1} \cdots s_{n}$, and consider the multiplication map

$$
\mathcal{H}_{\left\langle s_{1}\right\rangle}(X) \otimes \cdots \otimes \mathcal{H}_{\left\langle s_{n}\right\rangle}(X) \rightarrow \mathcal{H}_{W}(X)
$$

Every term in the resulting expansion corresponds to an element in which some (possibly empty) subset of the simple reflections have been omitted, and thus the image of this multiplication map is contained in $\mathcal{H}_{W}(X)[\leq w] \subset \mathcal{H}_{W}(X)[I]$. The image under the leading coefficient map can then be determined by replacing each factor by its corresponding leading coefficient line bundle. It thus remains only to verify that

$$
D_{w}=\sum_{1 \leq i \leq n} s_{1} \cdots s_{i-1}\left[X^{s_{i}}\right]=\sum_{1 \leq i \leq n}\left[X^{s_{1} \cdots s_{i-1} s_{i} s_{i-1} \cdots s_{1}}\right] .
$$

But this follows from the strong exchange property: the reflections $r$ such that $r w<w$ are precisely those of the form $s_{1} \cdots s_{i-1} s_{i} s_{i-1} \cdots s_{1}$, and these are distinct since such reflections are naturally bijective with the $n=\ell(w)$ positive roots that become negative under $w$.

Corollary 4.17. For any reduced word $w=s_{1} \cdots s_{n}$, the multiplication map

$$
\mathcal{H}_{\left\langle s_{1}\right\rangle}(X) \otimes \cdots \otimes \mathcal{H}_{\left\langle s_{n}\right\rangle}(X) \rightarrow \mathcal{H}_{W}(X)[\leq w]
$$

is surjective. Moreover, any product of rank 1 subalgebras is equal to some Bruhat interval.

Proof. It suffices to show that if $s w<w$, then

$$
\mathcal{H}_{\langle s\rangle} \otimes \mathcal{H}_{W}(X)[\leq s w] \rightarrow \mathcal{H}_{W}(X)[\leq w]
$$

is surjective. The image clearly contains the subsheaf $\mathcal{H}_{W}(X)[\leq s w]$, so it suffices to show surjectivity to the quotient $\mathcal{H}_{W}(X)[\leq w] / \mathcal{H}_{W}(X)[\leq s w]$. This, in turn, is an iterated extension of invertible sheaves $\mathcal{O}_{X}\left(D_{w^{\prime}}\right)$ on $X$, and thus it suffices to show surjectivity for each subquotient. That is, if $[\leq s w] \subset I \subset[\leq w]$ is an order ideal and $w^{\prime}$ is a maximal element of $I$ not contained 
in $[\leq s w]$, then we need to show that the intersection of the image with $\mathcal{H}_{W}(X)[I]$ surjects onto $\mathcal{L}_{D_{w^{\prime}}}$.

Since $s w<w$, we may choose a reduced word for $w$ beginning with $s$, and the subword description of the Bruhat order then tells us that $[\leq w]=[\leq s w] \cup s[\leq s w]$. Since $w^{\prime} \not \leq s w$ and $w^{\prime} \neq w$, it follows that $s w^{\prime}<s w$. We may thus consider the composition

$$
\mathcal{H}_{\langle s\rangle}(X) \otimes \mathcal{H}_{W}(X)\left[\leq s w^{\prime}\right] \rightarrow \mathcal{H}_{W}(X)\left[\leq w^{\prime}\right] \rightarrow \mathcal{H}_{W}(X)[I] .
$$

The proof of the lemma shows that the composition with the "coefficient of $w^{\prime \prime}$ map is surjective as required.

The second claim follows immediately from the fact that for any word $s_{1} \cdots s_{l}$, the products of subwords still form a Bruhat interval (so multiplication maps into the corresponding subsheaf), and the maximum of that interval can be represented by a reduced subword (so multiplication surjects).

Remark. In particular, the closest thing to an analogue of the braid relations in this setting is the fact that if $\left(s_{i} s_{j}\right)^{m_{i j}}=1$, then the products

$$
\mathcal{H}_{\left\langle s_{i}\right\rangle}(X) \mathcal{H}_{\left\langle s_{j}\right\rangle}(X) \cdots=\mathcal{H}_{\left\langle s_{j}\right\rangle}(X) \mathcal{H}_{\left\langle s_{i}\right\rangle}(X) \cdots
$$

(with $m_{i j}$ terms on each side) agree as subsheaves of $\mathcal{H}_{W}(X)$. Indeed, both sides are equal to the order ideal generated by the longest element of $\left\langle s_{i}, s_{j}\right\rangle$, and thus equal $\mathcal{H}_{\left\langle s_{i}, s_{j}\right\rangle}(X)$.

Corollary 4.18. The construction $\mathcal{H}_{W}(X)$ respects base change $T \rightarrow S$.

Proof. Let $\pi_{1}: X \times_{S} T \rightarrow X$ be the natural projection; we need to show that $\pi_{1}^{*} \mathcal{H}_{W}(X) \cong$ $\mathcal{H}_{W}\left(X \times_{S} T\right)$. In the rank 1 case, this is immediate from the explicit description and the fact that $\pi_{1}^{*}\left(\mathcal{O}_{X}\left(\left[X^{s}\right]\right)\right) \cong \mathcal{O}_{X}\left(\left[\left(X \times_{S} T\right)^{s}\right]\right)$. Since the rank 1 subalgebras generate the full algebra, this induces a morphism $\pi_{1}^{*} \mathcal{H}_{W}(X) \rightarrow \mathcal{H}_{W}\left(X \times_{S} T\right)$. (Normally one would need to check relations, but this is simply the restriction of the corresponding isomorphism for the algebra of meromorphic operators such that the common polar divisor does not contain any fiber; thus generators suffice.)

It remains only to show that this morphism is an isomorphism, but this follows from the existence of compatible filtrations (i.e., coming from a chain of Bruhat order ideals) such that the induced maps on subquotients are isomorphisms.

We can also give an alternate description of the adjoint involution. Let $w_{0}$ be the longest element of $W$.

Proposition 4.19. There is a contravariant isomorphism $\mathcal{H}_{W}(X)^{\mathrm{op}} \cong \mathcal{O}_{X}\left(-D_{w_{0}}\right) \otimes \mathcal{H}_{W}(X) \otimes$ $\mathcal{O}_{X}\left(D_{w_{0}}\right)$.

Proof. It suffices to show that the naïve adjoint $\sum_{w} c_{w} w \mapsto \sum_{w} w^{-1} c_{w}$ on the meromorphic twisted group algebra restricts to an isomorphism as claimed. Since this is an involution, it reduces to showing the analogous claim for each rank 1 subalgebra. Let $U$ be an open subset on which the effective Cartier divisor $D_{w_{0}}$ is cut out by an equation $h=0$. We thus need to show (using the two descriptions of the rank 1 Hecke algebra and taking the adjoint on the left)

$$
\Gamma\left(U ; \mathcal{O}_{X}\right)+\left(s_{i}-1\right) \Gamma\left(U ; \mathcal{O}_{X}\left(\left[C^{s}\right]\right)\right) \subset h\left(\Gamma\left(U ; \mathcal{O}_{X}\right)+\left(s_{i}+1\right) \Gamma\left(U ; \mathcal{L}\left[C^{s}\right]\right)\right) h^{-1} .
$$

Given an instance $f_{0}+\left(s_{i}-1\right) f_{1}$ on the left, conjugating by $h$ gives

$$
f_{0}-\left(1+\left(h /{ }^{s_{i}} h\right)\right) f_{1}+\left(s_{i}+1\right)\left(h /{ }^{s_{i}} h\right) f_{1} .
$$

Since ${ }^{s_{i}} D_{w_{0}}=D_{w_{0}}$, we find that $h /{ }^{s_{i}} h$ is a unit, and local considerations near $\left[X^{s_{i}}\right]$ tell us that $1+\left(h /{ }^{s_{i}} h\right)$ vanishes on $\left[X^{s_{i}}\right]$. 
Remark 4.20. We could also show that $\omega_{X} \otimes \mathcal{O}_{X}\left(D_{w_{0}}\right)$ descends in codimension 1 . The divisor $D_{w_{0}}$ is certainly invariant under every reflection, and thus it remains only to verify the conditions along reflection hypersurfaces. In characteristic not $2, \omega_{X}$ is equivariantly isomorphic to the twist of $\mathcal{O}_{X}$ by the sign character, and thus the condition is that the divisor must have odd valuation along the reflection hypersurfaces, while in characteristic 2 , there is no need to twist, and the valuations of separable reflection hypersurfaces must be even. In either case, the condition is automatically satisfied.

Remark 4.21. It is worth noting that this operation is triangular, in the sense that the image of the subsheaf corresponding to an order ideal is always the subsheaf corresponding to an order ideal. This follows immediately from the fact that $w \mapsto w^{-1}$ is an order-preserving automorphism of the Bruhat poset.

The proof of Theorem 3.9 has the following consequence for our algebras. Here and below, by the root kernel of $X$, we mean the root kernel of the corresponding abelian scheme $A$.

Proposition 4.22. Let $X / S$ be a flat family of abelian torsors equipped with a faithful action by reflections of the finite Weyl group $W$, with $\operatorname{dim}(X / S)=\operatorname{rank}(W)$. If the root kernel of $X$ is diagonalizable on $S$, then $S$ may be covered by open subsets on which $\mathcal{H}_{W}(X)$ has a symmetric idempotent: an idempotent global section which on each fiber has image $\Gamma\left(X_{x} ; \mathcal{O}_{X}\right)^{W}$.

Proof. For any fiber $x$, choose a global section $h$ of $\mathcal{O}_{X}\left(D_{w_{0}}\right)$ with nonzero symmetrization (reflections negate the fiber over 0 , so this corresponds to the antisymmetrization of Theorem 3.9), extend it to a neighborhood of $x \in S$, and observe that the antisymmetrization will remain nonzero in a possibly smaller, but nonempty, neighborhood. (By the proof of Theorem 3.9, this is guaranteed to exist for any geometric fiber (over which we can equivariantly trivialize the torsor), but the existence of an element with nontrivial antisymmetrization in an extension field implies the existence of such an element over the ground field.) Dividing by the symmetrization gives a function $f$ with poles at most $\Delta$ such that $\sum_{w \in W}{ }^{w} f=1$. The proof of Theorem 3.9 shows that the idempotent operator $\sum_{w \in W} w f$ preserves the space of functions holomorphic on any given invariant open subset of $X$, and thus is a section of $\mathcal{H}_{W}(X)$ over the given open subset of $S$.

Remark. Of course, we can always ensure the dim $=$ rank condition holds by taking the parameter space to be $X / A_{W}$. This condition is needed so that $\sum_{r \in R(W)}\left[X^{r}\right]$ is ample, allowing Theorem 3.9 to be applied. For instance, in the case of $A_{1}$ acting on $E^{2}$ by swapping the factors, every global section of $\mathcal{H}_{A_{1}}\left(E^{2}\right)$ has holomorphic coefficients, and thus in characteristic 2 there is no symmetric idempotent. The same argument gives a weaker statement without the dimension condition: for any ample divisor $D$ on $X / W$, there is (locally on $S$ ) a symmetric idempotent in $\Gamma\left(X / W ; \mathcal{H}_{W}(X) \otimes \mathcal{O}_{X / W}(D)\right)$.

Since $\mathcal{H}_{W}(X)$ contains the twisted group algebra of naïvely holomorphic operators, there is in particular an action of the group on any $\mathcal{H}_{W}(X)$-module, and thus for any such module $M$ which is (quasi)coherent as an $\mathcal{O}_{X / W}$-module, we could consider the $W$-invariant subsheaf of $M$. This as it stands may not be well-behaved, say for torsion sheaves supported on the reflection hypersurfaces. To obtain a better notion, we note that if we view $\mathcal{O}_{X}$ as a module over $\mathcal{H}_{W}(X)$, then there is a surjective $\mathcal{H}_{W}(X)$-module morphism $\sum_{w} c_{w} w \mapsto \sum_{w} c_{w}$ from $\mathcal{H}_{W}(X)$ to $\mathcal{O}_{X}$, with kernel containing the kernel of the natural morphism $\mathcal{O}_{X}[W] \rightarrow \mathcal{O}_{X}$. Thus for modules which are torsion-free as $\mathcal{O}_{X}$-modules, the sheaf $\mathcal{H}_{\mathcal{O}_{W}(X)}\left(\mathcal{O}_{X}, M\right)$ will agree with the sheaf of $W$ invariant sections of $M$. With this in mind, we define $M^{W}$ as the image of $\mathcal{H o m}_{\mathcal{H}_{W}(X)}\left(\mathcal{O}_{X}, M\right)$ under the "evaluate at 1" morphism. 
Corollary 4.23. If the root kernel of $X$ is diagonalizable, then the functor $-{ }^{W}$ on $\mathcal{H}_{W}(X)$ modules is exact and commutes with base change.

Proof. If $\mathcal{H}_{W}(X)$ has an idempotent of the form $\left(\sum_{w} w\right) h$, then the map $\mathcal{H}_{W}(X) \rightarrow \mathcal{O}_{X}$ (taking $\sum_{w} c_{w} w$ to $\left.\sum c_{w}\right)$ splits as $f \mapsto f\left(\sum_{w} w\right) h$. Since such idempotents exist locally on $S^{\prime}=X / A_{W}$, it follows that $\mathcal{O}_{X}$ is locally projective, and thus the corresponding sheaf Hom functor is exact. Moreover, it follows that $M^{W}=\left(\sum_{w} w\right) h M$, and this operation clearly commutes with base change.

Of course, as it stands, the algebra $\mathcal{H}_{W}(X)$ does not bear a terribly strong resemblance to the more familiar Hecke algebras, due to the lack of any parameters associated to the roots. Classically, one generally has one parameter for each orbit of roots, but in the classical $C_{n}$ case (viewing the affine Hecke algebra as being specified by an action of the finite Hecke algebra on the space of Laurent polynomials), one effectively has two parameters associated to the endpoint of the Dynkin diagram. This is traditionally interpreted as arising from the nonreduced root system $B C_{n}$, in which the endpoint is associated to two orbits of roots (differing by a factor of 2). If one looks at the actual action on Laurent polynomials, however, one finds that there is more symmetry in the parameters than is suggested by this interpretation, making it far more natural to associate an unordered pair of parameters to the given simple reflection. In fact, as we mentioned above, for our application, we will need a place to put an unbounded number of parameters; since there is already an example in which one can assign two parameters to a root without breaking things, this suggests that we should be able to assign arbitrarily many parameters to each orbit of roots.

Let us first consider the case of rank 1 , so that $X$ is a flat family $C / S$ of hyperelliptic curves of genus 1. By consideration of the classical $A_{1}$ and $C_{1}$ cases, we are led to consider the following algebra.

Definition 4.24. Let $C / S$ be a flat family of hyperelliptic curves of genus 1 on which $A_{1}=\langle s\rangle$ acts as the marked involution, and let $T$ be an effective Cartier divisor on $C$ not containing any fiber of $C$ over $S$. The rank 1 Hecke algebra $\mathcal{H}_{A_{1}, T}(C)$ is the subsheaf of $\mathcal{H}_{A_{1}}(C)$ such that the coefficient of $s$ in a local section of $\mathcal{H}_{A_{1}, T}(C)$ is a local section of $\mathcal{O}_{C}\left(\left[C^{s}\right]-T\right)$.

To see that this is an algebra, we note that the local sections of $\mathcal{H}_{A_{1}, T}(C)$ are precisely the operators of the form $f_{0}+f_{1}(s-1)$ with $f_{0} \in \Gamma\left(U ; \mathcal{O}_{C}\right), f_{1} \in \Gamma\left(U ; \mathcal{O}_{C}\left(\left[C^{s}\right]-T\right)\right.$, or equivalently the operators of the form $g_{0}+(s+1)^{s} g_{1}$ with $g_{0} \in \Gamma\left(U ; \mathcal{O}_{C}\right), g_{1} \in \Gamma\left(U ; \mathcal{O}_{C}\left(\left[C^{s}\right]-T\right)\right)$. Thus the general product of two local sections can be expressed as

$$
\begin{aligned}
\left(f_{0}+f_{1}(s-1)\right)\left(g_{0}+(s+1)^{s} g_{1}\right)= & f_{0} g_{0}+f_{1}\left({ }^{s} g_{0}-g_{0}\right)+f_{0}\left(g_{1}+{ }^{s} g_{1}\right) \\
& +\left(f_{1}{ }^{s} g_{0}+f_{0} g_{1}\right)(s-1),
\end{aligned}
$$

so that the coefficient of $s$ in the product is again a section of $\mathcal{O}_{C}\left(\left[C^{s}\right]-T\right)$.

There is an alternate description which makes the algebra property clearer, at the cost of a mild loss of generality.

Proposition 4.25. The algebra $\mathcal{H}_{A_{1}, T}(C)$ is contained in the subalgebra of $\mathcal{H}_{A_{1}}(C)$ which preserves the subsheaf $\mathcal{O}_{C}(-T) \subset \mathcal{O}_{C}$. If the divisors $T$ and ${ }^{s} T$ have no component in common, then this subalgebra is equal to $\mathcal{H}_{A_{1}, T}(C)$.

Proof. Let $U$ be an invariant open subset on which $T$ is cut out by a single equation $h=0$. Then the space $\Gamma\left(U ; \mathcal{H}_{A_{1}, T}(C)\right)$ can be described as the space of operators $f_{0}+(s+1) f_{1} s h$ such that $f_{0} \in \Gamma\left(U ; \mathcal{O}_{C}\right), f_{1} \in \Gamma\left(U ; \mathcal{O}_{C}\left(\left[C^{s}\right]\right)\right)$. Similarly, $\Gamma\left(U ; \mathcal{O}_{C}(-T)\right)=h \Gamma\left(U ; \mathcal{O}_{C}\right)$, so we need to 
show that $f_{0}+(s+1) f_{1} s h$ preserves $h \Gamma\left(U ; \mathcal{O}_{C}\right)$, or equivalently that $h^{-1}\left(f_{0}+(s+1) f_{1} s h\right) h \in$ $\Gamma\left(U ; \mathcal{H}_{A_{1}}(C)\right)$. Since

$$
h^{-1}\left(f_{0}+(s+1) f_{1}{ }^{s} h\right) h=f_{0}+h^{-1}(s+1) f_{1}\left({ }^{s} h h\right)=f_{0}+{ }^{s} h(s+1) f_{1},
$$

and $f_{0},{ }^{s} h,(s+1) f_{1} \in \Gamma\left(U ; \mathcal{H}_{A_{1}}(C)\right)$, the first claim follows.

Conversely, if $f_{0}+s f_{1} \in \Gamma\left(U ; \mathcal{H}_{A_{1}}(C)\right)$ also preserves $\left.\mathcal{O}_{C}(-T)\right|_{U}$, then both $f_{0}+s f_{1}$ and $h^{-1}\left(f_{0}+s f_{1}\right) h$ are in $\Gamma\left(U ; \mathcal{H}_{A_{1}}(C)\right)$. The first condition implies $f_{1} \in \Gamma\left(U ; \mathcal{O}_{C}\left(\left[C^{s}\right]\right)\right)$, while the second implies $f_{1} \in \Gamma\left(U ; \mathcal{O}_{C}\left(\left[C^{s}\right]-T+{ }^{s} T\right)\right.$. If ${ }^{s} T$ has no component in common with $T$, so that $\mathcal{O}_{C}(T) \cap \mathcal{O}_{C}\left({ }^{s} T\right)=\mathcal{O}_{C}$, then $\mathcal{O}_{C}\left(\left[C^{s}\right]\right) \cap \mathcal{O}_{C}\left(\left[C^{s}\right]-T+{ }^{s} T\right)=\mathcal{O}_{C}\left(\left[C^{s}\right]-T\right)$. In other words, $f_{1} \in \Gamma\left(U ; \mathcal{O}_{C}\left(\left[C^{s}\right]-T\right)\right)$, so that $f_{0}+s f_{1} \in \Gamma\left(U ; \mathcal{H}_{A_{1}, T}(C)\right)$ as required.

Remark. It is likely that the condition on $T$ here is slightly stronger than strictly necessary: the claim most likely continues to hold as long as $T \cap{ }^{s} T$ is contained in $\left(X^{s}\right)^{\text {red }}$.

Note that we could have used this to prove the algebra property, in the following way. For each nonnegative integer $m$, let $S^{\prime}$ be the relative symmetric $m$-th power of $C$ over $S$, and let $C^{\prime}$ be the base change of $C$ to $S^{\prime}$. There is a corresponding tautological divisor $T^{\prime}$, and our original data $(C / S, T)$ (assuming $T$ has degree $m$ over $S$ ) is the base change of $\left(C^{\prime} / S^{\prime}, T^{\prime}\right)$ by the section $S \rightarrow S^{\prime}$ corresponding to $T$. The space of operators as described respects base change, and thus it suffices to prove the algebra property in this larger family. Since $T^{\prime}$ and ${ }^{s} T^{\prime}$ have no component in common, this follows from the above result. There is one caveat here, though: although our original description respects base change, the description from the proposition does not. Indeed, if there is an effective divisor $T_{0}$ such that $T-T_{0}-{ }^{s} T_{0}$ is effective, then the subalgebra preserving $\mathcal{O}_{C}(-T)$ is the same as that preserving $\mathcal{O}_{C}\left(-T+T_{0}+{ }^{s} T_{0}\right)$, but the corresponding rank 1 Hecke algebras are not the same.

In the above argument, we used the fact that ${ }^{s} h h$ is central. This means we could also have described $\mathcal{H}_{A_{1}, T}(C)$ (subject to the given condition on $T$ ) as the subalgebra of $\mathcal{H}_{A_{1}}(C)$ preserving the supersheaf $\mathcal{O}_{C}\left({ }^{s} T\right)$. This symmetry leads to the following.

Proposition 4.26. There is a natural isomorphism

$$
\mathcal{O}_{C}(T) \otimes \mathcal{H}_{A_{1}, T}(C) \otimes \mathcal{O}_{C}(-T) \cong \mathcal{H}_{A_{1},{ }^{s} T}(C) .
$$

Proof. Replacing $(C / S, T)$ by a larger family as necessary, we may assume that $T$ and ${ }^{s} T$ have no component in common. We then have

$$
\mathcal{H}_{A_{1}, T}(C)=\mathcal{H}_{A_{1}}(C) \cap \mathcal{O}_{C}(-T) \otimes \mathcal{H}_{A_{1}}(C) \otimes \mathcal{O}_{C}(T)
$$

and thus, conjugating by $\mathcal{O}_{C}(T)$,

$$
\mathcal{O}_{C}(T) \otimes \mathcal{H}_{A_{1}, T}(C) \otimes \mathcal{O}_{C}(-T)=\mathcal{H}_{A_{1}}(C) \cap \mathcal{O}_{C}(T) \otimes \mathcal{H}_{A_{1}}(C) \otimes \mathcal{O}_{C}(-T)
$$

Replacing $T$ by ${ }^{s} T$ in the alternate description

$$
\mathcal{H}_{A_{1}, T}(C)=\mathcal{H}_{A_{1}}(C) \cap \mathcal{O}_{C}\left({ }^{s} T\right) \otimes \mathcal{H}_{A_{1}}(C) \otimes \mathcal{O}_{C}\left(-{ }^{s} T\right)
$$

tells us that

$$
\mathcal{H}_{A_{1}}(C) \cap \mathcal{O}_{C}(T) \otimes \mathcal{H}_{A_{1}}(C) \otimes \mathcal{O}_{C}(-T)=\mathcal{H}_{A_{1},{ }^{s} T}(C)
$$

as required. 
Proposition 4.27. The adjoint isomorphism $\mathcal{H}_{A_{1}}(C)^{\mathrm{op}} \cong \mathcal{O}_{C}\left(-\left[C^{s}\right]\right) \otimes \mathcal{H}_{A_{1}}(C) \otimes \mathcal{O}_{C}\left(\left[C^{s}\right]\right)$ restricts to a contravariant isomorphism

$$
\mathcal{H}_{A_{1}, T}(C)^{\mathrm{op}} \cong \mathcal{O}_{C}\left(-\left[C^{s}\right]\right) \otimes \mathcal{H}_{A_{1},{ }^{s} T}(C) \otimes \mathcal{O}_{C}\left(\left[C^{s}\right]\right),
$$

inducing a contravariant isomorphism

$$
\mathcal{H}_{A_{1}, T}(C)^{\mathrm{op}} \cong \mathcal{O}_{C}\left(T-\left[C^{s}\right]\right) \otimes \mathcal{H}_{A_{1}, T}(C) \otimes \mathcal{O}_{C}\left(\left[C^{s}\right]-T\right)
$$

With this construction in mind, let $\vec{T}$ be a system of effective Cartier divisors $T_{\alpha}$ on $X$ associated to the roots $\alpha \in \Phi(W)$, such that $T_{\alpha}$ never contains a fiber of $X$ and $w\left(T_{\alpha}\right)=T_{w \alpha}$ for all $\alpha \in \Phi(W), w \in W$. Clearly, to specify such a system, it suffices to specify $T_{\alpha}$ for one representative of each orbit of roots, subject to the condition that $w\left(T_{\alpha}\right)=T_{\alpha}$ whenever $w \alpha=\alpha$. Although the construction would work in this generality, we will also impose the further condition that $T_{\alpha}$ descends to a divisor on the corresponding coroot curve (or, equivalently, is invariant under translation by any point in $\left.\left(1+r_{\alpha}\right) A\right)$. This makes the stabilizer condition automatic, and thus we may specify $\vec{T}$ by specifying effective divisors on the coroot curves associated to a set of inequivalent simple roots.

We will call such a system $\vec{T}$ of divisors a "system of parameters for $W$ on $X$ ".

Definition 4.28. Let $W$ be a finite Weyl group acting on an abelian torsor $X / S$ by reflections, and let $\vec{T}$ be a system of parameters for $W$ on $X$. Then the Hecke algebra $\mathcal{H}_{W ; \vec{T}}(X)$ is the subalgebra of $\mathcal{H}_{W}(X)$ generated by the rank 1 algebras $\mathcal{H}_{\left\langle s_{i}\right\rangle, T_{i}}(X)$.

We again have a filtration by Bruhat order, inherited from $\mathcal{H}_{W}(X)$, and the subquotients are again explicit line bundles.

Lemma 4.29. Let I be a Bruhat order ideal, and suppose that $w$ is a maximal element of $I$. Then there is a short exact sequence

$$
0 \rightarrow \mathcal{H}_{W ; \vec{T}}(X)[I \backslash\{w\}] \subset \mathcal{H}_{W ; \vec{T}}(X)[I] \rightarrow \mathcal{O}_{X}\left(D_{w}(\vec{T})\right) \rightarrow 0
$$

where $D_{w}(\vec{T}):=\sum_{r \in R(W), r w<w}\left(\left[X^{r}\right]-T_{\alpha_{r}}\right)$, with $\alpha_{r}$ the positive root corresponding to $r$.

Proof. Suppose first that $T_{\alpha}$ never has a component in common with the discriminant divisor $D_{w_{0}}$. Then an easy induction tells us that the left coefficient of $w$ in any local section of $\mathcal{H}_{W ; \vec{T}}(X)$ vanishes on $T_{\alpha_{r}}$ for every reflection $r$ such that $r w<w$; this is by a calculation as in Lemma 4.16 above, except that we must also argue that $s_{1} \cdots s_{i-1} \alpha_{i}$ is positive. But this is again standard Coxeter theory; if it were not positive, then $s_{1} \cdots s_{i}$ could not be a reduced word. The claim then follows as in the no parameter case.

To extend this to bad parameters, we observe (as in the rank 1 case, as we will discuss more precisely below) that we can always embed our family in a larger family which generically satisfies the condition on $\vec{T}$. On the one hand, since $\mathcal{H}_{W ; \vec{T}}(X)$ is generated by a flat family of submodules, its Hilbert polynomial is lower semicontinuous and is thus bounded above by the sum of the Hilbert polynomials of the line bundles of the subquotients of the generic Bruhat filtration. Since we can construct elements of Bruhat intervals with the desired leading coefficients, it follows that this bound must be tight, and the claim follows in general.

Remark. We may also write the divisor as $D_{w}(\vec{T})=\sum_{\alpha \in \Phi^{+}(W) \cap w \Phi^{-}(W)}\left(\left[X^{r_{\alpha}}\right]-T_{\alpha}\right)$.

This leads to an alternate description valid under fairly weak conditions on the system of parameters. 
Corollary 4.30. Suppose $\vec{T}$ is such that every $T_{\alpha}$ is transverse to every reflection hypersurface. Then $\mathcal{H}_{W ; \vec{T}}(X)$ may be identified with the subalgebra of $\mathcal{H}_{W}(X)$ consisting of local sections $\sum_{w} c_{w} w$ such that $c_{w}$ vanishes on $\sum_{\alpha \in \Phi^{+}(W) \cap w \Phi^{-}(W)} T_{\alpha}$ for every $w \in W$.

Proof. We showed in the proof of Lemma 4.29 that every section of $\mathcal{H}_{W ; \vec{T}}(X)$ satisfies the given vanishing conditions, so it remains only to show that every local section of $\mathcal{H}_{W}(X)$ satisfying the conditions is in fact a local section of $\mathcal{H}_{W ; \vec{T}}(X)$. Let $\mathcal{D}=\sum_{w} c_{w}$ be such a section (on the open subset $U \subset X / W)$, and $I$ be the smallest order ideal containing the support of $\mathcal{D}$, with $w_{1}$ a maximal element of $I$. Then $c_{w_{1}}$ is a section of $\mathcal{O}_{X}\left(D_{w_{1}}(\vec{T})\right)$, so that by Lemma 4.29 , there is an open covering $U=\cup_{i} V_{i}$ such that on each $V_{i}$ there is a local section of $\mathcal{H}_{W ; \vec{T}}(X)$ supported on $I$ with the same left coefficient of $w_{1}$. Subtracting this local section gives an element which by induction is itself a local section of $\mathcal{H}_{W ; \vec{T}}(X)$. It follows that the restriction of $\mathcal{D}$ to each $V_{i}$ is a section of $\mathcal{H}_{W ; \vec{T}}(X)$, and thus $\mathcal{D}$ is a local section of $\mathcal{H}_{W ; \vec{T}}(X)$ as required.

Similarly, the corollaries carry over immediately.

Corollary 4.31. For any reduced word $w=s_{1} \cdots s_{n}$, the multiplication map

$$
\mathcal{H}_{\left\langle s_{1}\right\rangle, \vec{T}}(X) \otimes \cdots \otimes \mathcal{H}_{\left\langle s_{n}\right\rangle, \vec{T}}(X) \rightarrow \mathcal{H}_{W ; \vec{T}}(X)[\leq w]
$$

is surjective. Moreover, any product of rank 1 subalgebras is equal to some Bruhat interval.

Corollary 4.32. The construction $\mathcal{H}_{W ; \vec{T}}(X)$ respects base change.

We also have an immediate extension of the adjoint isomorphism.

Proposition 4.33. The adjoint isomorphism $\mathcal{H}_{W}(X)^{\mathrm{op}} \cong \mathcal{O}_{X}\left(-D_{w_{0}}\right) \otimes \mathcal{H}_{W}(X) \otimes \mathcal{O}_{X}\left(D_{w_{0}}\right)$ restricts to a contravariant isomorphism

$$
\mathcal{H}_{W ; \vec{T}}(X)^{\mathrm{op}} \cong \mathcal{O}_{X}\left(-D_{w_{0}}(\vec{T})\right) \otimes \mathcal{H}_{W ; \vec{T}}(X) \otimes \mathcal{O}_{X}\left(D_{w_{0}}(\vec{T})\right)
$$

Proof. Again, it suffices to prove that the adjoint identifies the corresponding rank 1 subalgebras, and one finds that twisting by $\mathcal{O}_{X}\left(D_{w_{0}}(\vec{T})-D_{s_{i}}(\vec{T})\right)$ has no effect, so the claim follows from the rank 1 case.

One important special case is when $T_{\alpha}=\left[X^{r_{\alpha}}\right]$ (which descends to the coroot curve since it is the preimage of the identity under the composition $X \rightarrow E_{r}^{\prime} \rightarrow E_{r}$ ). In that case, we find that the rank 1 subalgebras are just the twisted group algebras $\mathcal{O}_{X}[\langle s\rangle]$, and thus that the full algebra is itself simply equal to $\mathcal{O}_{X}[W]$.

One disadvantage of the approach via rank 1 subalgebras is that it is not particularly convenient when trying to determine whether a given operator is a (local) section of the Hecke algebra. For this, it will be helpful to have a generalization of Proposition 4.25.

Proposition 4.34. The algebra $\mathcal{H}_{W ; \vec{T}}(X)$ is contained in the subalgebra of $\mathcal{H}_{W}(X)$ preserving the subsheaf $\mathcal{O}_{X}\left(-\sum_{\alpha \in \Phi^{+}(W)} T_{\alpha}\right) \subset \mathcal{O}_{X}$, with equality holding unless there is a root $\alpha$ such that $T_{\alpha}$ and $T_{-\alpha}$ have a common component.

Proof. Containment reduces to showing that the rank 1 subalgebras preserve the given subsheaf. Since the simple reflection $s_{i}$ permutes the positive roots other than $\alpha_{i}$, the divisor $T_{i}-\sum_{\alpha \in \Phi^{+}(W)} T_{\alpha}$ is $s_{i}$-invariant, and has trivial valuation along the components of $\left[X^{s_{i}}\right]$. It follows that on the corresponding rank 1 subalgebra, preserving $\mathcal{O}_{X}\left(-\sum_{\alpha \in \Phi^{+}(W)} T_{\alpha}\right)$ is equivalent to preserving $\mathcal{O}_{X}\left(-T_{i}\right)$, at which point the claim is just Proposition 4.25. 
Using the Bruhat filtration, we see that equality holds whenever

$$
\mathcal{O}_{X}\left(D_{w}(\vec{T})\right)=\mathcal{O}_{X}\left(D_{w}\right) \cap \mathcal{O}_{X}\left(D_{w}-\sum_{\alpha \in \Phi^{+}(W)} T_{\alpha}+w\left(\sum_{\alpha \in \Phi^{+}(W)} T_{\alpha}\right)\right) .
$$

Since

$$
\sum_{\alpha \in \Phi^{+}(W)} T_{\alpha}-w\left(\sum_{\alpha \in \Phi^{+}(W)} T_{\alpha}\right)=\sum_{\alpha \in \Phi^{+}(W)} T_{\alpha}-\sum_{\alpha \in \Phi^{+}(W)} T_{w \alpha}=\sum_{\alpha \in \Phi^{+}(W) \cap w \Phi^{-}(W)}\left(T_{\alpha}-T_{-\alpha}\right),
$$

we have equality as long as there is no cancellation, i.e., unless there is a positive root $\alpha$ and a negative root $\beta$ such that $T_{\alpha}$ and $T_{\beta}$ have a common component. If $\beta \neq-\alpha$, then the two divisors are pulled back through different coroot maps, and thus cannot have a common component, so only the case $T_{\alpha}, T_{-\alpha}$ is relevant, and the claim follows.

As in the rank 1 case, the restriction on the divisors is not particularly serious, as we can always obtain the algebra we want as the base change of a more general family. In particular, if $S^{\prime}$ is an appropriate product of relative symmetric powers of coroot curves, then there is a corresponding tautological system of parameters $\vec{T}^{\prime}$ on the base change to $S^{\prime}$, and the original system $\vec{T}$ is the pullback along a suitable section $S \rightarrow S^{\prime}$.

Corollary 4.35. There is a natural isomorphism

$$
\mathcal{O}_{X}\left(\sum_{\alpha \in \Phi^{+}(W)} T_{\alpha}\right) \otimes \mathcal{H}_{W ; \vec{T}}(X) \otimes \mathcal{O}_{X}\left(-\sum_{\alpha \in \Phi^{+}(W)} T_{\alpha}\right) \cong \mathcal{H}_{W ;-\vec{T}}(X),
$$

where $^{-} T_{\alpha}:=T_{-\alpha}$.

Another source of isomorphisms is diagram automorphisms.

Corollary 4.36. Let $\delta$ be an automorphism of $X$ over $S$ such that composition with $\delta$ permutes the set of positive coroot maps. Then $\delta$ normalizes $W$, and the induced action on $\mathcal{H}_{W}(X)$ preserves $\mathcal{H}_{W ; \vec{T}}(X)$ for all $\vec{T}$.

Proof. The assumption on $\delta$ (and finiteness of $W$ ) implies that $\delta$ preserves the set of simple coroot maps as well as the divisor $\sum_{\alpha \in \Phi^{+}(W)} T_{\alpha}$.

Corollary 4.37. Let $w_{0}$ be the longest element of $W$. Then the action of $w_{0}$ on $\mathcal{H}_{W}(X)$ takes $\mathcal{H}_{W ; \vec{T}}$ to $\mathcal{H}_{W ;-\vec{T}}$.

Proof. Since $[-1] w_{0}$ acts as a diagram automorphism, it suffices to show the corresponding fact for [-1], which clearly commutes with $W$ and satisfies

$$
[-1]\left(\sum_{\alpha \in \Phi^{+}(W)} T_{\alpha}\right)=\sum_{\alpha \in \Phi^{+}(W)} T_{-\alpha} .
$$

We now turn to modules over $\mathcal{H}_{W ; \vec{T}}(X)$. Since we constructed $\mathcal{H}_{W ; \vec{T}}(X)$ as a space of operators, this gives rise to a natural left module denoted $\mathcal{O}_{X}$, which as a sheaf on $X / W$ is the direct image of $\mathcal{O}_{X}$. This works more generally for any $W$-equivariant line bundle $\mathcal{L}$ that descends in codimension 1 , as $\mathcal{H}_{W ; 0}(X)$ still acts on such bundles. (We also have a corresponding submodule $\mathcal{L}\left(-\sum_{\alpha \in \Phi^{+}(W)} T_{\alpha}\right)$ coming from Proposition 4.34, which we will discuss more below.)

An important construction of modules comes from the fact that our algebras are generated by the rank 1 subalgebras, and thus any parabolic subgroup $W_{I}$ induces a corresponding 
parabolic subalgebra $\mathcal{H}_{W_{I} ;\left.\vec{T}\right|_{\Phi\left(W_{I}\right)}}(X) \subset \mathcal{H}_{W ; \vec{T}}(X)$, which by mild abuse of notation we denote by $\mathcal{H}_{W_{I} ; \vec{T}}(X)$. As a result, given a (left) $\mathcal{H}_{W_{I} ; \vec{T}}(X)$-module $M$, we may tensor with $\mathcal{H}_{W ; \vec{T}}(X)$ to obtain an induced $\mathcal{H}_{W ; \vec{T}}(X)$-module which we denote by $\operatorname{Ind}_{W_{I}}^{W ; \vec{T}} M$, or by $\operatorname{Ind}_{W_{I}}^{W ; 0} M$ when considering the analogous construction for the master Hecke algebra; we also denote the corresponding restriction functor as $\operatorname{Res}_{W_{I}}^{W ; \vec{T}}$. Note that the restriction of the left module associated to a line bundle is the left module associated to the same line bundle.

If $\mathcal{L}$ is a $W$-equivariant line bundle on $\mathcal{O}_{X}$ that descends in codimension 1 , then we have a locally free module $\operatorname{Ind}_{1}^{W ; \vec{T}} \mathcal{L}$, which establishes a Morita autoequivalence of the category of $\mathcal{H}_{W ; \vec{T}}(X)$-modules, which we denote by $\mathcal{L} \otimes-$. We of course have a corresponding notion for right modules, with $\operatorname{Ind}_{1}^{W ; \vec{T}} \mathcal{L} \cong \mathcal{L} \otimes \mathcal{H}_{W ; \vec{T}}(X) \cong \mathcal{H}_{W ; \vec{T}}(X) \otimes \mathcal{L}$. Similarly, there is an equivalence $\mathcal{L}\left(-\sum_{\alpha \in \Phi^{+}(W)} T_{\alpha}\right) \otimes-$ taking $\mathcal{H}_{W ; \vec{T}}(X)$-modules to $\mathcal{H}_{W ;-\vec{T}}(X)$-modules.

If we take the restriction of an induced module, we would ordinarily expect the result to split as a sum over double cosets. This fails even in the case of the regular representation, as $\mathcal{H}_{W ; \vec{T}}(X)$ does not naturally split as a direct sum of $\left(\mathcal{H}_{W_{I} ; \vec{T}}(X), \mathcal{H}_{W_{J} ; \vec{T}}(X)\right)$-bimodules corresponding to double cosets. The case $I=J=\varnothing$ is suggestive however: although the Hecke algebra does not split as a sum of line bundles indexed by $W$, our results on the Bruhat filtration come fairly close. It turns out that there is a natural Bruhat order on (parabolic) double cosets. Indeed, every double coset $W_{I} w W_{J}$ has a unique minimal representative, and the restriction of Bruhat order to the set of such representatives is well-behaved. (See, e.g., [36] and references therein.) In particular, for any order ideal in the set ${ }^{I} W^{J}$ of minimal representatives, the corresponding union of double cosets is an order ideal in $W$. As a result, any order ideal in ${ }^{I} W^{J}$ induces a corresponding sub-bimodule of $\mathcal{H}_{W ; \vec{T}}(X)$, and thus a subfunctor of $\operatorname{Res}_{W_{I} ; \vec{T}} \operatorname{Ind}_{W_{J}}^{W ; \vec{T}}$.

Given any element $w \in{ }^{I} W^{J}$, the intersections $W_{I} \cap w W_{J} w^{-1}$ and $w^{-1} W_{I} w \cap W_{J}$ are both parabolic, giving subsets $I(w) \subset I, J(w) \subset J$ such that $W_{I(w)} \cong W_{J(w)}$, extending in an obvious way to an isomorphism of the corresponding Hecke algebras.

Lemma 4.38. For any $w \in{ }^{I} W^{J}, D_{w}(\vec{T})$ is $W_{I(w)}$-invariant, and has trivial valuation along the corresponding reflection hyperplanes.

Proof. We recall the expression

$$
D_{w}(\vec{T})=\sum_{\alpha \in \Phi^{+}(W) \cap w \Phi^{-}(W)}\left(\left[X^{r_{\alpha}}\right]-T_{\alpha}\right) .
$$

The fact that $w$ is $W_{I}$-minimal implies that no root of $W_{I}$ appears in this sum, and thus in particular that no root of $W_{I(w)}$ appears. It thus remains only to show $W_{I(w)}$-invariance, but this follows by comparing $D_{s_{i} w}(\vec{T})$ and $D_{w s_{j}}(\vec{T})$ for reflections $s_{i} \in W_{I}, s_{j} \in W_{J}$ such that $s_{i} w=w s_{j}$.

This ensures that the twisting functor in the following Mackey-type result is well-defined.

Proposition 4.39. Let $I, J \subset S$. Then for any $\mathcal{H}_{W_{J} ; \vec{T}}(X)$-module $M$ and any maximal chain in the Bruhat order on ${ }^{I} W^{J}$, the subquotient corresponding to $w \in{ }^{I} W^{J}$ in the resulting filtration of $\operatorname{Res}_{W_{I}}^{W ; \vec{T}} \operatorname{Ind}_{W_{J}}^{W ; \vec{T}} M$ is the $\mathcal{H}_{W_{I} ; \vec{T}}(X)$-module

$$
\operatorname{Ind}_{W_{I(w)}}^{W_{I} ; \vec{T}}\left(\mathcal{O}_{X}\left(D_{w}(\vec{T})\right) \otimes w \operatorname{Res}_{W_{J(w)} ; \vec{T}}^{W_{J}} M\right)
$$

where here $w$ represents the induced isomorphism from the category of $\mathcal{H}_{W_{J(w)}, \vec{T}}(X)$-modules to the category of $\mathcal{H}_{W_{I(w)}, \vec{T}}(X)$-modules. 
Proof. Since the description of the subquotient is functorial, it suffices to consider the case that $M=\mathcal{H}_{W_{J} ; \vec{T}}(X)$, or in other words to consider the Bruhat filtration on $\mathcal{H}_{W ; \vec{T}}(X)$ viewed as a bimodule. Let $O, O \cup\{w\}$ be the elements of the chosen maximal chain that differ by $w$, so that we need to understand the quotient of the subsheaf corresponding to $W_{I}(O \cup\{w\}) W_{J}$ by the subsheaf corresponding to $W_{I} O W_{J}$. Both of these are bimodules over the respective Hecke algebras, and the actions commute with projecting onto the vector space of meromorphic operators supported on $W_{I} w W_{J}$. We thus immediately see from Corollary 4.31 that the quotient is generated by the subsheaf supported on $W_{I(w)} w W_{J(w)}=W_{I(w)} w$, and is in fact induced from the corresponding $\left(\mathcal{H}_{W_{I(w)}, \vec{T}}(X), \mathcal{H}_{W_{J(w)}, \vec{T}}(X)\right)$-bimodule structure. Moreover, one easily verifies that this bimodule induces the Morita equivalence $M \mapsto \mathcal{O}_{X}\left(D_{w}(\vec{T})\right) \otimes w M$, from which the result follows. Note that the fact that $w \in{ }^{I} W^{J}$ ensures that $D_{w}(\vec{T})$ is $W_{I(w)}$-invariant and has trivial valuation along the reflection hypersurfaces corresponding to $R\left(W_{I(w)}\right)$, so this twisting is indeed well-defined.

Taking $I=\varnothing$ gives the following, where we omit $\varnothing$ from the notation in ${ }^{\varnothing} W^{J}$.

Corollary 4.40. Let $I \subset S$. Then for any $\mathcal{H}_{W_{I} ; \vec{T}}(X)$-module $M$ and any maximal chain in the Bruhat order on $W^{I}$, the subquotient corresponding to $w \in W^{I}$ in the resulting filtration of $\operatorname{Ind}_{W_{I}}^{W ; \vec{T}} M$ is the $\mathcal{O}_{X}$-module $\mathcal{O}_{X}\left(D_{w}(\vec{T})\right) \otimes{ }^{w} M$.

Corollary 4.41. The functor $\operatorname{Ind}_{W_{I}}^{W ; \vec{T}}$ is exact.

Proof. Indeed, the proof of Proposition 4.39 shows that the $\mathcal{H}_{W_{I} ; \vec{T}}(X)$-module $\mathcal{H}_{W ; \vec{T}}(X)$ has a filtration by (locally) free modules, so is itself locally projective.

For finite groups, this exactness arises from the fact that restriction and induction are adjoint in both directions. This is again not quite true in our setting, but something fairly close is true. Define

$$
\operatorname{Coind}_{W_{I}}^{W ; \vec{T}} M:=\mathcal{H}_{\mathcal{O}_{W_{I} ; \vec{T}}}(X)\left(\mathcal{H}_{W ; \vec{T}}(X), M\right),
$$

which is clearly right adjoint to $\operatorname{Res}_{W_{I}}^{W ; \vec{T}}$. Given a set $I$ of simple roots, let $I^{\prime}$ denote its image under the diagram automorphism corresponding to $w_{0}$, and note that the double coset $W_{I^{\prime}} w_{0} W_{I}=w_{0} W_{I}=W_{I^{\prime}} w_{0}$. Let $w_{I}$ denote the longest element of $W_{I}$.

Lemma 4.42. For any $\mathcal{H}_{W_{I} ; \vec{T}}(X)$-module $M$, there is a natural isomorphism

$$
\operatorname{Ind}_{W_{I}}^{W ; \vec{T}}(M) \cong \operatorname{Coind}_{W_{I^{\prime}} ; \vec{T}}^{W}\left(\mathcal{O}_{X}\left(D_{w_{0} w_{I}}(\vec{T})\right) \otimes w_{0} w_{I} M\right) .
$$

Proof. There is a natural morphism

$$
\operatorname{Res}_{W_{I^{\prime}}}^{W ; \vec{T}} \operatorname{Ind}_{W_{I}}^{W ; \vec{T}}(M) \rightarrow \mathcal{O}_{X}\left(D_{w_{0} w_{I}}(\vec{T})\right) \otimes w_{0} w_{I} M
$$

since the codomain is precisely the top subquotient in the Bruhat filtration of the domain. By adjunction, this induces a morphism from the induced module to the coinduced module, and it remains only to show that this is an isomorphism. The image of a local section $x \in \operatorname{Ind}_{W_{I}}^{W ; \vec{T}}(M)$ in the coinduced module is the map taking local sections $y \in \operatorname{Res}_{W_{I^{\prime}} ; \vec{T}} \mathcal{H}_{W ; \vec{T}}(X)$ to the top subquotient of $y x$ in the $W_{I^{\prime}} \backslash W / W_{I}$ filtration. The map $w \mapsto w_{0} w_{I} w^{-1}$ induces an orderreversing isomorphism from $W^{I}$ to ${ }^{I^{\prime}} W$, and thus our putative isomorphism is triangular, and it suffices to show that it is an isomorphism on the diagonal. This reduces to showing that

$$
D_{w_{0} w_{I} w^{-1}}(\vec{T})+{ }^{w_{0} w_{I} w^{-1}} D_{w}(\vec{T})=D_{w_{0} W_{I}}(\vec{T})
$$


for any $w \in W^{I}$, which in turn reduces to $\ell\left(w_{0} w_{I} w^{-1}\right)+\ell(w)=\ell\left(w_{0} W_{I}\right)$ and thus to $\ell\left(w w_{I}\right)=$ $\ell(w)+\ell\left(W_{I}\right)$.

Remark. Since the transformation being applied to $M$ is invertible, we also have an expression

$$
\operatorname{Coind}_{W_{I}}^{W ; \vec{T}} M \cong \operatorname{Ind}_{W_{I^{\prime}}}^{W ; \vec{T}}\left(\mathcal{O}_{X}\left(-D_{w_{0} w_{I}}(-\vec{T})\right) \otimes w_{0} w_{I} M\right)
$$

As in the master Hecke algebra case, we again have a module $\mathcal{O}_{X}$ coming from the action on operators, and the restriction to $\mathcal{H}_{W ; \vec{T}}(X)$ of the natural map $\mathcal{H}_{W}(X) \rightarrow \mathcal{O}_{X}$ is still surjective. In particular, we may again define $M^{W}$ to be the image of the natural injective morphism $\mathcal{H o m}_{\mathcal{H}_{W ; \vec{T}}(X)}\left(\mathcal{O}_{X}, M\right) \rightarrow M$.

Proposition 4.43. The kernel of the natural morphism $\mathcal{H}_{W ; \vec{T}}(X) \rightarrow \mathcal{O}_{X}$ is generated as a left ideal sheaf by the subsheaves of the form $\mathcal{O}_{X}\left(\left[X^{s_{i}}\right]-T_{i}\right)\left(s_{i}-1\right)$.

Proof. Let $\mathcal{I}$ be the left ideal sheaf so generated. This is clearly contained in the kernel, so it remains to show that it contains the kernel. Let $\sum_{w} c_{w} w$ be a local section of the kernel, and suppose $w_{1}$ is Bruhat-maximal among the elements of $W$ for which $c_{w} \neq 0$. Since by definition $\sum_{w} c_{w}=0, w_{1}$ cannot be the identity, and thus has a reduced expression of the form $w_{1}=s_{1} \cdots s_{m}$ with $m>0$. We thus have a (surjective) multiplication map

$$
\mathcal{H}_{\left\langle s_{1}\right\rangle, \vec{T}}(X) \cdots \mathcal{H}_{\left\langle s_{m}\right\rangle, \vec{T}}(X) \rightarrow \mathcal{H}_{W ; \vec{T}}(X)\left[\leq w_{1}\right] .
$$

Restricting the last tensor factor to $\mathcal{O}_{X}\left(\left[X^{s_{m}}\right]-T_{m}\right)\left(s_{m}-1\right)$ gives an image in $\mathcal{I}$ without changing the leading coefficient line bundle, and thus there is an element $\sum_{w \leq w_{1}} c_{w}^{\prime} w$ of $\mathcal{I}$ with $c_{w}^{\prime}=c_{w}$. Subtracting this element makes the order ideal generated by the support of the operator smaller, and thus the result follows by induction.

Corollary 4.44. There is an exact sequence of $\mathcal{O}_{X / W}$-modules

$$
0 \rightarrow M^{W} \rightarrow M \rightarrow \bigoplus_{1 \leq i \leq n} \mathcal{O}_{X}\left(T_{i}-\left[X^{s_{i}}\right]\right) \otimes M
$$

Proof. The proposition gives a presentation of $\mathcal{O}_{X}$, and this is just the sheaf $\mathcal{H}$ om from that presentation to $M$.

If $M$ is $S$-flat, then this tells us that $M^{W}$ is the kernel of a morphism of $S$-flat sheaves.

Lemma 4.45. Let $Y / S$ be a projective morphism with relatively ample line bundle $\mathcal{O}_{Y}(1)$, and suppose $\phi: M \rightarrow N$ is a morphism of $S$-flat coherent sheaves on $Y$. If the Hilbert polynomial of $\operatorname{ker}\left(\phi_{s}\right)$ is independent of the point $s \in S$, then the kernel, image, and cokernel of $\phi$ are all $S$-flat, and the natural map $\operatorname{ker}(\phi)_{s} \rightarrow \operatorname{ker}\left(\phi_{s}\right)$ is an isomorphism for all $s$.

Proof. If the Hilbert polynomial of $\operatorname{ker}\left(\phi_{s}\right)$ is independent of $s$, then so is the Hilbert polynomial of $\operatorname{coker}\left(\phi_{s}\right) \cong \operatorname{coker}(\phi)_{s}$. It follows that $\operatorname{coker}(\phi)$ is $S$-flat, implying immediately that the image and kernel are also $S$-flat (as kernels of surjective morphisms of $S$-flat sheaves). The final claim follows using the four-term sequence

$$
0 \rightarrow \operatorname{Tor}_{2}(\operatorname{coker}(\phi), k(s)) \rightarrow \operatorname{ker}(\phi)_{s} \rightarrow \operatorname{ker}\left(\phi_{s}\right) \rightarrow \operatorname{Tor}_{1}(\operatorname{coker}(\phi), k(s)) \rightarrow 0
$$

arising by comparing the two spectral sequences for tensoring the complex $M \rightarrow N$ with $k(s)$.

Corollary 4.46. If $M$ is a coherent $S$-flat $\mathcal{H}_{W: \vec{T}}(X)$-module such that the Hilbert polynomial of $\left(M_{s}\right)^{W}$ is independent of $s$, then $M^{W}, M / M^{W}$ are flat and the natural map $\left(M^{W}\right)_{s} \rightarrow\left(M_{s}\right)^{W}$ is an isomorphism for all $s$. 
If $M$ satisfies the hypothesis, we say that $M$ has "strongly flat invariants".

Before introducing parameters, we could show that this functor respected base change and flatness by observing that (subject to diagonalizability of the root kernel) the Hecke algebra had (locally on $X / A_{W}$ ) idempotents projecting onto $M^{W}$. Unfortunately, this fails, and quite badly, in cases with parameters. Indeed, if the divisors $T_{\alpha}$ are of sufficiently large degree, then the subquotient corresponding to $w$ in the Bruhat filtration will have negative degree unless $w$ is the identity, and thus in such a case the fibers of the Hecke algebra cannot have any nonscalar global sections, let alone symmetric idempotents.

Luckily, $S$-flatness is local on the source, not the base, and thus the correct condition is not that there be global symmetric idempotents, but merely that there be local symmetric idempotents.

Lemma 4.47. Let $U$ be a $W$-invariant open subset. If $h \in \Gamma\left(U ; \mathcal{O}_{X}\left(D_{w_{0}}(\vec{T})\right)\right)$, then

$$
\left(\sum_{w} w\right)^{w_{0}} h \in \Gamma\left(U ; \mathcal{H}_{W ; \vec{T}}(X)\right) .
$$

Proof. Suppose first that $T_{\alpha}$ and $T_{-\alpha}$ have no common component for any root $\alpha$, so that we may use Proposition 4.34 to characterize $\mathcal{H}_{W ; \vec{T}}(X)$. The given operator clearly maps $\Gamma\left(V ; \mathcal{O}_{X}\right)$ to $\Gamma\left(U \cap V ; \mathcal{O}_{X}\right)^{W}$ for any invariant open $V$, so that $\left(\sum_{w} w\right)^{w_{0}} h \in \mathcal{H}_{W}(X)$. Similarly, if $f \in \Gamma\left(V ;-\sum_{\alpha \in \Phi^{+}(W)} T_{\alpha}\right)$, then ${ }^{w_{0}} h f \in \Gamma\left(V ; D_{w_{0}}-\sum_{\alpha \in \Phi(W)} T_{\alpha}\right)$, so that the symmetrization vanishes along $\sum_{\alpha \in \Phi(W)} T_{\alpha}$. The claim follows in this case.

For the general case, we base change from the family with universal $\vec{T}$, and observe that since $D_{w_{0}}(\vec{T})$ is a flat family of divisors, $h$ extends to a local section of $\mathcal{O}_{X}\left(D_{w_{0}}(\vec{T})\right)$ on a neighborhood of the original base. We thus find that there is a local section of the larger Hecke algebra that restricts to the desired local section, from which the result follows.

We say that $\mathcal{H}_{W ; \vec{T}}(X)$ has a local symmetric idempotent at a point $x \in X / W$ if the restriction of $\mathcal{H}_{W ; \vec{T}}(X)$ to the local ring at $x$ contains an idempotent of the form $\left(\sum_{w} w\right) h$. By the lemma, this is equivalent to asking for the restriction of $\mathcal{O}_{X}\left(D_{w_{0}}(\vec{T})\right)$ to the local ring at the orbit corresponding to $x$ to contain an element $h$ with $\sum_{w \in W}{ }^{w} h=1$. Moreover, if there is an element for which this sum is a unit, then we can divide by the sum to obtain an element symmetrizing to 1 . It follows that if the condition holds on the fiber containing $x$, then we still have a local symmetric idempotent at $x$; that is, the condition of having a local symmetric idempotent respects base change.

Similarly, we say that $\mathcal{H}_{W ; \vec{T}}(X)$ is covered by symmetric idempotents if it has a local symmetric idempotent at every point $x \in X / W$. This is too much to hope for even without imposing parameters, as the $A_{2}$ example we considered at the end of Section 3 gives an explicit point where the master Hecke algebra fails to have a local symmetric idempotent. In general, the most we can say is that there is a (possibly empty) open subset of $S$ such that the base change is covered by symmetric idempotents. Indeed, the condition to have a symmetric idempotent at $x$ is open, and $X / S$ is proper, so we can simply take the complement of the image of the complement of the locus with local symmetric idempotents.

Lemma 4.48. Suppose that the root kernel of $X$ is diagonalizable, and that for any nonnegative linear dependence $\sum_{i} k_{i} \alpha_{i}=0$ of roots, the intersection $\cap_{i} T_{\alpha_{i}}$ is empty. Then $\mathcal{H}_{W ; \vec{T}}(X)$ is covered by symmetric idempotents.

Proof. This is local in $S$, so we may restrict to an open subset over which $\mathcal{H}_{W, 0}(X)$ has a global symmetric idempotent $\sum_{w} w h$; diagonalizability of the root kernel ensures that these open 
subsets cover $S$. For any point $x \in X$, let $D_{x}$ be the corresponding decomposition group, and observe that

$$
1=\sum_{w \in W}{ }^{w} h=\sum_{g \in D_{x}} g\left(\sum_{w \in D_{x} \backslash W}{ }^{w} h\right),
$$

and thus there is a section of $\mathcal{O}_{X}\left(D_{w_{0}}\right)$ in the local ring at $x$ for which the sum over the decomposition group is 1 .

Now, suppose that $x$ is not contained in $T_{\alpha}$ for any positive $\alpha$. Then this local section of $\mathcal{O}_{X}\left(D_{w_{0}}\right)$ at $x$ is in fact a section of $\mathcal{O}_{X}\left(D_{w_{0}}-\sum_{\alpha} T_{\alpha}\right)$ near $x$, and we can add a section that vanishes at $x$ in such a way that the resulting section is holomorphic on the orbit $W x$ and vanishes at the points of the orbit other than $x$. It follows that the resulting function symmetrizes to a unit in the relevant local ring, and thus gives rise to a local symmetric idempotent at $W x$.

In general, let $\Phi_{x}$ be the set of roots such that $x \in T_{\alpha}$. Since $x$ is contained in the corresponding intersection of divisors $T_{\alpha}$, we conclude that the elements of $\Phi_{x}$, viewed as real vectors, cannot satisfy any nonnegative linear dependence. This implies that there is a real linear functional which is negative on $\Phi_{x}$, and thus (since all systems of positive roots in a finite Weyl group are equivalent) that $w \Phi_{x} \subset \Phi^{-}(X)$ for some $w \in W$. This implies that $w x$ satisfies the conditions for the construction of the previous paragraph to apply, and thus that there is a local symmetric idempotent in a neighborhood of the orbit $W x$.

It is not too hard to see that the empty intersection condition is satisfied on the generic fiber of the family with universal $\vec{T}$; indeed, if we further base change to express each $T_{i}$ as a sum of points, then the values of those parameters at a point of such an intersection must themselves satisfy a nonnegative linear dependence, and there are only finitely many minimal such dependences to consider. It follows that any family of Hecke algebras is the base change of a family which is generically covered by symmetric idempotents.

Lemma 4.49. If $\mathcal{H}_{W ; \vec{T}}(X)$ is covered by symmetric idempotents, then $-{ }^{W}$ is exact and any coherent $\mathcal{H}_{W ; \vec{T}}(X)$-module $M$ has strongly flat invariants.

Proof. Indeed, $\mathcal{O}_{X}$ is locally a direct summand of $\mathcal{H}_{W \cdot \vec{T}}(X)$, and is therefore locally projective as before. Exactness follows immediately. Any local section of $\left(M_{s}\right)^{W}$ on an open subset supporting a symmetric idempotent extends to a local section of $M$ which can then be projected to a section of $M^{W}$ restricting to the given section of $\left(M_{s}\right)^{W}$. It follows that the natural map $\left(M^{W}\right)_{s} \rightarrow\left(M_{s}\right)^{W}$ is an isomorphism. Since $M^{W}$ is locally a direct summand of $M$, it is flat, and thus its fibers have constant Hilbert polynomial as required.

It turns out that if the generic fiber is covered by symmetric idempotents, then this has consequences even on those fibers without such a covering.

Lemma 4.50. Suppose that there is a dense open subset of $S$ over which $\mathcal{H}_{W ; \vec{T}}(X)$ is covered by symmetric idempotents, and suppose that the module $M$ admits a filtration such that each subquotient is $S$-flat with strongly flat invariants. Then $M$ has strongly flat invariants.

Proof. Fix a relatively ample bundle $\mathcal{O}_{X / W}(1)$ on $X / W$. By semicontinuity, for any point $s \in S$ and $d \gg 0$, we have

$$
\begin{aligned}
\operatorname{dim}\left(\Gamma\left(X / W,\left(M_{k(S)}\right)^{W}(d)\right)\right) & \leq \operatorname{dim}\left(\Gamma\left(X / W,\left(M_{s}\right)^{W}(d)\right)\right) \\
& \leq \sum_{i} \operatorname{dim}\left(\Gamma\left(X / W,\left(M_{s}^{i}\right)^{W}(d)\right)\right) \\
& =\sum_{i} \operatorname{dim}\left(\Gamma\left(X / W,\left(M_{k(S)}^{i}\right)^{W}(d)\right)\right),
\end{aligned}
$$


where the $M^{i}$ are the subquotients of the given filtration on $M$. Since the generic fiber of $\mathcal{H}_{W ; \vec{T}}(X)$ is covered by symmetric idempotents, $-{ }^{W}$ is exact on the generic fiber and thus

$$
\operatorname{dim}\left(\Gamma\left(X / W,\left(M_{k(S)}\right)^{W}(d)\right)\right)=\sum_{i} \operatorname{dim}\left(\Gamma\left(X / W,\left(M_{k(S)}^{i}\right)^{W}(d)\right)\right)
$$

for $d \gg 0$, implying

$$
\operatorname{dim}\left(\Gamma\left(X / W,\left(M_{k(S)}\right)^{W}(d)\right)\right)=\operatorname{dim}\left(\Gamma\left(X / W,\left(M_{s}\right)^{W}(d)\right)\right)
$$

as required.

To apply this, we will need a family of modules for which we can prove strongly flat invariants without resorting to idempotents. For $I \subset S$, let $w_{I}$ denote the maximal element of $W_{I}$.

Proposition 4.51. Let $I \subset S$ and let $\mathcal{L}$ be a $W_{I}$-equivariant line bundle that descends in codimension 1. Then we have a natural isomorphism $\left(\operatorname{Ind}_{W_{I}}^{W} \mathcal{L}\right)^{W} \cong\left(\mathcal{L}\left(D_{w_{0}}\left({ }^{-} \vec{T}\right)-D_{w_{I}}(-\vec{T})\right)\right)^{W_{I}}$ of $\mathcal{O}_{X / W}$-modules, where the right-hand side denotes the sheaf of $W_{I}$-invariant sections of the given line bundle.

Proof. For any $\mathcal{H}_{W_{I} ; \vec{T}}(X)$-module $M$, we have

$$
\begin{aligned}
\left(\operatorname{Ind}_{W_{I}}^{W ; \vec{T}} M\right)^{W} & \cong\left(\operatorname{Coind}_{W_{I^{\prime}} ; \vec{T}}\left(\mathcal{O}_{X}\left(D_{w_{0} w_{I}}(\vec{T})\right) \otimes w_{0} w_{I} M\right)\right)^{W} \\
& \cong\left(\mathcal{O}_{X}\left(D_{w_{0} w_{I}}(\vec{T})\right) \otimes w_{0} w_{I} M\right)^{W_{I^{\prime}}} \\
& \cong\left(\mathcal{O}_{X}\left({ }^{w_{I} w_{0}} D_{w_{0} w_{I}}(\vec{T})\right) \otimes M\right)^{W_{I}} \\
& \cong\left(\mathcal{O}_{X}\left(D_{w_{0}}(-\vec{T})-D_{w_{I}}(-\vec{T})\right) \otimes M\right)^{W_{I}}
\end{aligned}
$$

We may thus reduce to the case $W=W_{I}$, where the result is immediate.

Corollary 4.52. Suppose that the root kernel of $X$ is diagonalizable. Then any module of the form $M=\operatorname{Ind}_{W_{I}}^{W} \mathcal{L}$ has strongly flat invariants.

Corollary 4.53. Let $I, J \subset S$ and let $\mathcal{L}_{I}, \mathcal{L}_{J}$ be $W_{I}, W_{J}$-equivariant line bundles that descend in codimension 1 . If the root kernel of $X$ is diagonalizable, then

$$
\mathcal{H} \operatorname{om}_{\mathcal{H}_{W ; \vec{T}}(X)}\left(\operatorname{Ind}_{W_{I}}^{W ; \vec{T}} \mathcal{L}_{I}, \operatorname{Ind}_{W ;}^{W ; \vec{T}} \mathcal{L}_{J}\right)
$$

is $S$-flat and respects base change.

Proof. By extending the family as appropriate (noting that $\mathcal{L}_{I}$ and $\mathcal{L}_{J}$ themselves extend), we may assume that there is a dense open subset $U \subset S$ which is covered by symmetric idempotents. We observe that

$$
\begin{aligned}
\mathcal{H}_{\mathcal{O}_{W ; \vec{T}}(X)}\left(\operatorname{Ind}_{W_{I}}^{W ; \vec{T}} \mathcal{L}_{I}, \operatorname{Ind}_{W ;}^{W ; \vec{T}} \mathcal{L}_{J}\right) & \cong \mathcal{H}_{\mathcal{H}_{\mathcal{H}_{W_{I}} ; \vec{T}}(X)}\left(\mathcal{L}_{I}, \operatorname{Res}_{W_{I} ; \vec{T}}^{W} \operatorname{Ind}_{W_{J} ; \vec{T}}^{W} \mathcal{L}_{J}\right) \\
& \cong\left(\mathcal{L}_{I}^{-1} \otimes \operatorname{Res}_{W_{I} ; \vec{T}} \operatorname{Ind}_{W_{J}}^{W ; \vec{T}} \mathcal{L}_{J}\right)^{W_{I}} .
\end{aligned}
$$

Each subquotient of the Bruhat filtration for

$$
\mathcal{L}_{I}^{-1} \otimes \operatorname{Res}_{W_{I}}^{W ; \vec{T}} \operatorname{Ind}_{W_{J}}^{W ; \vec{T}} \mathcal{L}_{J}
$$

has strongly flat invariants, and thus the same holds for the $\mathcal{H}_{W_{I} ; \vec{T}}(X)$-module itself. It follows in particular that the module is $S$-flat and that the construction commutes with base change. 
Proposition 4.54. Let $\mathcal{L}_{I}, \mathcal{L}_{J}$ be $W_{I}, W_{J}$-equivariant line bundles that descend in codimension 1. If the root kernel of $X$ is diagonalizable, then there is a natural isomorphism

$$
\mathcal{H}_{\mathrm{om}_{\mathcal{H}_{W}(X)}}\left(\operatorname{Ind}_{W_{J}}^{W ; 0} \mathcal{L}_{J}, \operatorname{Ind}_{W_{I}}^{W ; 0} \mathcal{L}_{I}\right) \cong \mathcal{H} \operatorname{om}\left(\left(\pi_{*} \mathcal{L}_{I}\right)^{W_{I}},\left(\pi_{*} \mathcal{L}_{J}\right)^{W_{J}}\right)
$$

which is (contravariantly) compatible with composition.

Proof. Since the root kernel is diagonalizable, both $\mathcal{H}_{W_{I}}(X)$ and $\mathcal{H}_{W_{J}}(X)$ have symmetric idempotents $e_{I}, e_{J}$ on the complement of any $W$-invariant ample divisor, and these embed as local sections of $\mathcal{E} \operatorname{nd}\left(\pi_{*} \mathcal{L}_{I}\right)$ and $\mathcal{E} \operatorname{nd}\left(\pi_{*} \mathcal{L}_{J}\right)$ respectively. We may thus identify the sections of the left-hand side as the subspace $e_{J} \mathcal{H} \operatorname{om}\left(\pi_{*} \mathcal{L}_{I}, \pi_{*} \mathcal{L}_{J}\right) e_{I}$, and this is contravariant with respect to composition. As a section of $\mathcal{E} \operatorname{nd}\left(\pi_{*} \mathcal{L}_{I}\right), e_{I}$ is a projection onto $\left(\pi_{*} \mathcal{L}_{I}\right)^{W_{I}}$, and similarly for $e_{J}$; the claim follows immediately.

Remark 4.55. Note that if $\mathcal{L}_{I}$ descends to a line bundle on $X / W_{I}$, then $\left(\pi_{*} \mathcal{L}_{I}\right)^{W_{I}}$ may be identified with the direct image of that line bundle.

Remark 4.56. If $\mathcal{L}_{I}$ and $\mathcal{L}_{J}$ are trivial and $J=\varnothing$, then the right-hand side consists of operators locally taking $W_{I}$-invariant holomorphic functions to holomorphic functions, and may thus be identified with the sheaf $\mathcal{M}_{\vec{g} W_{I}}$, where $g_{1}, \ldots, g_{n}$ are a system of coset representatives for $W / W_{I}$. More generally, if $\sum_{w W_{I} \in W / W_{I}} c_{w W_{I}} w W_{I}$ is such an operator, requiring that the image be $W_{J}$-invariant is equivalent to requiring that $(g-1) \sum_{w W_{I} \in W / W_{I}} c_{w W_{I}} w W_{I}$ annihilate every $W_{I}$-invariant function, and thus that the operator itself is invariant under left multiplication by elements of $W_{J}$. Thus, on any invariant open subset, the given space is determined by $W_{J}$-invariance along with (by Corollary 4.8) the condition that the pole of $c_{w W_{I}}$ is bounded by the sum $\sum_{r: r w W_{I} \neq w W_{I}}\left[X^{r}\right]$, and the condition that for any reflection, $c_{w W_{I}}+c_{r w W_{I}}$ is holomorphic along $\left[X^{r}\right]$. If $\mathcal{L}_{I}$ and $\mathcal{L}_{J}$ are nontrivial, but $\mathcal{L}_{I}$ descends, then the first two conditions remain the same, but the residue condition becomes the holomorphy of ${ }^{w} f c_{w W_{I}}+{ }^{r w} f c_{r w W_{I}}$ for any $W_{I^{-}}$-invariant meromorphic section of $\mathcal{L}_{I}$ such that ${ }^{w} f$ is holomorphic on $\left[X^{r}\right]$. Here, it suffices to check the condition for a single section $f$ as long as both ${ }^{w} f$ and ${ }^{w} f^{-1}$ are holomorphic along $\left[X^{r}\right]$.

There is an analogue of the adjoint in this setting.

Corollary 4.57. If the root kernel of $X$ is diagonalizable, then there is an isomorphism

$$
\begin{aligned}
\mathcal{H}_{\mathcal{H}_{W}(X)} & \left(\operatorname{Ind}_{W_{J}}^{W ; 0} \mathcal{O}_{X}, \operatorname{Ind}_{W_{I}}^{W ; 0} \mathcal{O}_{X}\right) \\
& \cong \mathcal{H}_{\mathcal{H}_{W}(X)}\left(\operatorname{Ind}_{W_{I}}^{W ; 0} \mathcal{O}_{X}\left(D_{w_{0}}-D_{w_{I}}\right), \operatorname{Ind}_{W_{J}}^{W ; 0} \mathcal{O}_{X}\left(D_{w_{0}}-D_{w_{J}}\right)\right),
\end{aligned}
$$

contravariant with respect to composition.

Proof. Embedding the left-hand side in $\mathcal{E} \operatorname{nd}\left(\pi_{*} \mathcal{O}_{X}\right)$ and taking the adjoint there gives an isomorphism to $\mathcal{H} \operatorname{om}\left(e_{J}^{*} \pi_{*} \mathcal{O}_{X}\left(D_{w_{0}}\right), e_{I}^{*} \pi_{*} \mathcal{O}_{X}\left(D_{w_{0}}\right)\right)$, where $e_{I}^{*}, e_{J}^{*}$ are the adjoints of the corresponding idempotents. If $e_{I}=\left(\sum_{w \in W_{I}} w\right) h_{I}$, then $e_{I}^{*}=h_{I}\left(\sum_{w \in W_{I}} w\right)$, and we then find that

$$
e_{I}^{*} \pi_{*} \mathcal{O}_{X}\left(D_{w_{0}}\right)=h_{I}\left(\sum_{w \in W_{I}} w\right) \pi_{*} \mathcal{O}_{X}\left(D_{w_{0}}\right)=h_{I}\left(\pi_{*} \mathcal{O}_{X}\left(D_{w_{0}}-D_{w_{I}}\right)\right)^{W_{I}},
$$

where the second equality follows from Theorem 3.9. We thus have

$$
\begin{aligned}
\mathcal{H o m} & \left(e_{J}^{*} \pi_{*} \mathcal{O}_{X}\left(D_{w_{0}}\right), e_{I}^{*} \pi_{*} \mathcal{O}_{X}\left(D_{w_{0}}\right)\right) \\
& \cong \mathcal{H o m}\left(h_{J}\left(\pi_{*} \mathcal{O}_{X}\left(D_{w_{0}}-D_{w_{J}}\right)\right)^{W_{J}}, h_{I}\left(\pi_{*} \mathcal{O}_{X}\left(D_{w_{0}}-D_{w_{I}}\right)\right)^{W_{I}}\right) \\
& \cong \mathcal{H} \operatorname{om}\left(\left(\pi_{*} \mathcal{O}_{X}\left(D_{w_{0}}-D_{w_{J}}\right)\right)^{W_{J}},\left(\pi_{*} \mathcal{O}_{X}\left(D_{w_{0}}-D_{w_{I}}\right)\right)^{W_{I}}\right),
\end{aligned}
$$

from which the claim follows. 
Remark. There is, of course, a version with a pair of line bundles; we omit the details.

Define

$$
\mathcal{H}_{W, W_{I}, W_{J} ; \vec{T}}(X):=\mathcal{H}_{\mathrm{om}_{\mathcal{H}} ; \vec{T}}(X)\left(\operatorname{Ind}_{W_{J}}^{W ; \vec{T}} \mathcal{O}_{X}, \operatorname{Ind}_{W_{I}}^{W ; \vec{T}} \mathcal{O}_{X}\right),
$$

with composition law given by

$$
\mathcal{H}_{W, W_{J}, W_{K} ; \vec{T}}(X) \otimes \mathcal{H}_{W, W_{I}, W_{J} ; \vec{T}}(X) \rightarrow \mathcal{H}_{W, W_{I}, W_{K} ; \vec{T}}(X),
$$

contravariant to the standard composition on Hom sheaves.

Proposition 4.58. If the root kernel of $X$ is diagonalizable, then $\mathcal{H}_{W, W_{I}, W_{J} ; \vec{T}}(X)$ may be identified with a subsheaf of $\mathcal{H}_{W, W_{I}, W_{J}}(X)$, compatibly with composition. Moreover, the corresponding operators take $W_{I^{-}}$invariant sections of the line bundle $\mathcal{O}_{X}\left(\sum_{\alpha \in \Phi^{-}(W) \backslash \Phi^{-}\left(W_{I}\right)} T_{\alpha}\right)$ to $W_{J^{-}}$-invariant sections of the line bundle $\mathcal{O}_{X}\left(\sum_{\alpha \in \Phi^{-}(W) \backslash \Phi^{-}\left(W_{J}\right)} T_{\alpha}\right)$. Conversely, if there are no roots such that $T_{\alpha}$ and $T_{-\alpha}$ have a common component, then $\mathcal{H}_{W, W_{I}, W_{J} ; \vec{T}}(X)$ is precisely the subsheaf of $\mathcal{H}_{W, W_{I}, W_{J}}(X)$ cut out by this condition.

Proof. Suppose first that $\mathcal{H}_{W_{I} ; \vec{T}}(X)$ and $\mathcal{H}_{W_{J} ; \vec{T}}(X)$ are covered by symmetric idempotents. This allows us to (locally) embed $\mathcal{H}_{W, W_{I}, W_{J} ; \vec{T}}(X)$ in $\mathcal{H}_{W ; \vec{T}}(X)$ as in the parameter-free case. It follows that $\mathcal{H}_{W, W_{I}, W_{J} ; \vec{T}}(X)$ acts on the $W_{I}$-invariant sections of $k(X)$ in such a way as to take $W_{I}$-invariant sections of $\mathcal{O}_{X}$ to $W_{J}$-invariant sections of $\mathcal{O}_{X}$ and $W_{I}$-invariant sections

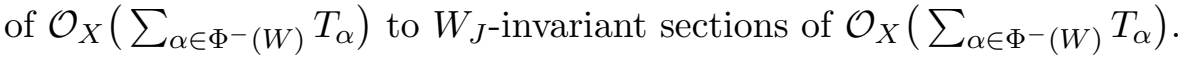

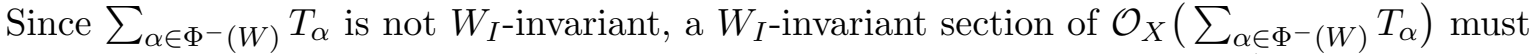
lie in the intersection of the images of this bundle under $W_{I}$, so in particular (taking the intersection with the image under $w_{I}$ ) in

$$
\mathcal{O}_{X}\left(\sum_{\alpha \in \Phi^{-}(W)} T_{\alpha}\right) \cap \mathcal{O}_{X}\left(\sum_{\alpha \in \Phi^{+}\left(W_{I}\right) \cup \Phi^{-}(W) \backslash \Phi^{-}\left(W_{I}\right)} T_{\alpha}\right),
$$

which by hypothesis is $\mathcal{O}_{X}\left(\sum_{\alpha \in \Phi^{-}(W) \backslash \Phi^{-}\left(W_{I}\right)} T_{\alpha}\right)$. The same calculation for $J$ tells us that the elements of $\mathcal{H}_{W, W_{I}, W_{J} ; \vec{T}}(X)$ act as required.

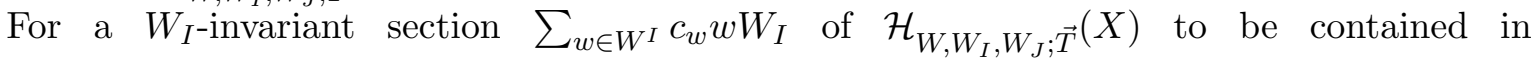
$\mathcal{H}_{W, W_{I}, W_{J}}(X)$ is a closed condition, and thus holds in general (extending to the family with universal parameters as necessary). That it respects the given supersheaves is also a closed condition, and thus the first claim follows for general parameters.

To show equality under the conditions on $T_{\alpha}$, it suffices to compare subquotients in the respective Bruhat filtrations, and thus to compute the intersection

$$
\mathcal{O}_{X} \cap \mathcal{O}_{X}\left(\sum_{\alpha \in \Phi^{-}} T_{\alpha} T_{\alpha}-\sum_{\left.\alpha \in \Phi^{-}(W) \backslash W_{J}\right)} T_{w \alpha}\right) .
$$

(More precisely, we want to intersect the $W_{J(w)}$-invariant subsheaves, but since both sheaves are equivariant, we may as well take their intersection before passing to invariants.) We may write

$$
\sum_{\alpha \in \Phi^{-}(W) \backslash \Phi^{-}\left(W_{J}\right)} T_{\alpha}-\sum_{\alpha \in \Phi^{-}(W) \backslash \Phi^{-}\left(W_{I}\right)} T_{w \alpha}=\sum_{\alpha \in \Phi^{-}(W)}\left(T_{\alpha}-T_{w \alpha}\right)-\sum_{\alpha \in \Phi^{-}\left(W_{J}\right)} T_{\alpha}+\sum_{\alpha \in \Phi^{-}\left(W_{I}\right)} T_{w \alpha} .
$$

Here

$$
\sum_{\alpha \in \Phi^{-}(W)}\left(T_{\alpha}-T_{w \alpha}\right)=\sum_{\alpha \in \Phi^{+}(W) \cap w \Phi^{-}(W)}\left(T_{-\alpha}-T_{\alpha}\right)
$$


while

$$
\sum_{\alpha \in \Phi^{-}\left(W_{I}\right)} T_{w \alpha}-\sum_{\alpha \in \Phi^{-}\left(W_{J}\right)} T_{\alpha}=\sum_{\alpha \in \Phi^{-}\left(W_{I}\right) \backslash \Phi^{-}\left(W_{I} \cap w^{-1} W_{J} w\right)} T_{w \alpha}-\sum_{\alpha \in \Phi^{-}\left(W_{J}\right) \backslash \Phi^{-}\left(W_{J} \cap w W_{I} w^{-1}\right)} T_{\alpha} .
$$

The hypotheses ensure that there is no further cancellation, so the intersection is

$$
\mathcal{O}_{X}\left(-\sum_{\alpha \in \Phi^{+}(W) \cap w \Phi^{-}(W)} T_{\alpha}-\sum_{\alpha \in \Phi^{-}\left(W_{J}\right) \backslash \Phi^{-}\left(W_{J} \cap w W_{I} w^{-1}\right)} T_{\alpha}\right),
$$

agreeing with the ( $T$-dependent part of the) line bundle arising in the Bruhat filtration.

Corollary 4.59. Let $\vec{T}$ be a system of parameters such that every $T_{\alpha}$ is transverse to every reflection hypersurface. If the root kernels of $W_{I}$ and $W_{J}$ are diagonalizable, then $\mathcal{H}_{W, W_{I}, W_{J} ; \vec{T} ; \gamma}(X)$ may be identified with the subsheaf of $\mathcal{H}_{W, W_{I}, W_{J} ; \gamma}(X)$ consisting of operators $\sum_{w} c_{w} w W_{I}$ such that for every $w, c_{w}$ vanishes on the divisor

$$
\sum_{\alpha \in \Phi^{+}(W) \cap w \Phi^{-}(W)} T_{\alpha}+\sum_{\alpha \in \Phi^{-}\left(W_{J}\right) \backslash \Phi^{-}\left(W_{J} \cap w W_{I} w^{-1}\right)} T_{\alpha} .
$$

Corollary 4.60. There is an isomorphism

$$
\begin{aligned}
\mathcal{H}_{W, W_{I}, W_{J} ; \vec{T}}(X) \cong & \mathcal{O}_{X}\left(D_{w_{0}}\left({ }^{-} \vec{T}\right)-D_{w_{J}}\left({ }^{-} \vec{T}\right)\right) \otimes \mathcal{H}_{W, W_{J}, W_{I} ; \vec{T}}(X) \\
& \otimes \mathcal{O}_{X}\left(D_{w_{I}}\left({ }^{-} \vec{T}\right)-D_{w_{0}}\left({ }^{-} \vec{T}\right)\right)
\end{aligned}
$$

which is contravariant for the natural composition.

Proof. Extend to universal parameters, write the left-hand side as an intersection, and take the adjoint of both twists of $\mathcal{H}_{W, W_{I}, W_{J} ; \vec{T}}(X)$. The resulting equality extends as usual to the full parameter space.

When $I=J$, we denote this by $\mathcal{H}_{W, W_{I} ; \vec{T}}(X)$, and call the resulting sheaf of algebras a spherical algebra of the Hecke algebra, which is in general a subalgebra of the algebra $\mathcal{E} \operatorname{nd}\left(\left(\pi_{*} \mathcal{L}_{I}\right)^{W_{I}}\right)$ corresponding to $\vec{T}=0$.

Proposition 4.58 leads in the usual way to a description of the spherical algebra for general $\vec{T}$ as an intersection of two twists of the master spherical algebra:

$$
\begin{aligned}
\mathcal{H}_{W, W_{I}, W_{J} ; \vec{T}}(X)=\mathcal{H}_{W, W_{I}, W_{J}}(X) & \\
& \cap \mathcal{O}_{X}\left(\sum_{\alpha \in \Phi^{-}(W) \backslash \Phi^{-}\left(W_{J}\right)} T_{\alpha}\right) \otimes \mathcal{H}_{W, W_{I}, W_{J}}(X) \otimes \mathcal{O}_{X}\left(-\sum_{\alpha \in \Phi^{-}(W) \backslash \Phi^{-}\left(W_{I}\right)} T_{\alpha}\right) .
\end{aligned}
$$

In the case of the Hecke algebra, we could twist this description to make the second term untwisted, and found a relation between the original Hecke algebra and the Hecke algebra for $-\vec{T}$. In this case, however, the resulting twisted bimodule is not itself a spherical bimodule, as the divisors

$$
\sum_{\alpha \in \Phi^{-}(W) \backslash \Phi^{-}\left(W_{I}\right)} T_{\alpha} \quad \text { and } \quad-\sum_{\alpha \in \Phi^{+}(W) \backslash \Phi^{+}\left(W_{I}\right)} T_{\alpha}
$$

do not differ by a $W$-invariant divisor. One can, however, give a somewhat related description for the resulting twist, using the other family of $\mathcal{H}_{W ; \vec{T}}(X)$-modules associated to line bundles. We find the following by tracing the various twists. 
Corollary 4.61. We have a natural isomorphism

$$
\begin{aligned}
\mathcal{H}_{\mathcal{H}_{W ; \vec{T}}(X)}\left(\operatorname{Ind}_{W_{J}}^{W ; \vec{T}} \mathcal{O}_{X}\left(-\sum_{\alpha \in \Phi^{+}\left(W_{J}\right)} T_{\alpha}\right), \operatorname{Ind}_{W_{I}}^{W ; \vec{T}} \mathcal{O}_{X}\left(-\sum_{\alpha \in \Phi^{+}\left(W_{I}\right)} T_{\alpha}\right)\right) \\
\cong \mathcal{O}\left(\sum_{\alpha \in \Phi^{-}(W) \cup \Phi^{+}\left(W_{J}\right)} T_{\alpha}\right) \otimes \mathcal{H}_{W, W_{I}, W_{J} ;-\vec{T}^{(X)}}(X) \mathcal{O}\left(-\sum_{\alpha \in \Phi^{-}(W) \cup \Phi^{+}\left(W_{I}\right)} T_{\alpha}\right),
\end{aligned}
$$

and in particular the left-hand side respects base change.

This suggests looking at what happens when only one of the two bundles is of the given form. Unfortunately, this behaves badly for special values of $\vec{T}$, as the piece associated to any given Bruhat subquotient involves taking $W$-invariant sections of a line bundle which is not itself equivariant, but instead a subbundle of an equivariant bundle cut out by (generically) non-invariant vanishing conditions. This of course is not a problem when there is a covering by symmetric idempotents, which suggests looking for an alternate description that gives the same bimodule on such fibers. This is not too difficult: when $\mathcal{H}_{W ; \vec{T}}(X)$ has a covering by symmetric idempotents, the module $\mathcal{O}_{X}$ is locally projective (and thus so is every module induced from it), while the $\mathcal{H}_{W ; \vec{T}}(X)$-module $\mathcal{O}_{X}\left(-\sum_{\alpha \in \Phi^{+}(W)} T_{\alpha}\right)$ is locally projective whenever $\mathcal{H}_{W ;-\vec{T}}(X)$ has a covering by symmetric idempotents. As a result, if $\mathcal{L}$ is a twist of either of these by an equivariant bundle, then the natural map

$$
\mathcal{H o m}_{\mathcal{H}_{W ; \vec{T}}(X)}\left(\mathcal{L}, \mathcal{H}_{W ; \vec{T}}(X)\right) \otimes M \rightarrow \mathcal{H} \operatorname{om}_{\mathcal{H}_{W ; \vec{T}}(X)}(\mathcal{L}, M)
$$

is an isomorphism. The tensor product still behaves badly in the cases of interest, but in a different way: for bad values of $\vec{T}$, it acquires torsion. Thus we may hope that the image of this natural map will give a strongly flat extension of the Hom sheaf from the symmetric idempotent locus. This image has a general description which is closely related to the description coming from symmetric idempotents when they exist.

Proposition 4.62. The image of the natural map

$$
\mathcal{H o m}_{\mathcal{H}_{W ; \vec{T}}(X)}\left(\mathcal{O}_{X}, \mathcal{H}_{W ; \vec{T}}(X)\right) \otimes_{\mathcal{H}_{W ; \vec{T}}(X)} M \rightarrow M^{W}
$$

is the same as the image of the map

$$
m \mapsto \sum_{w \in W} w m: \mathcal{O}_{X}\left(D_{w_{0}}(-\vec{T})\right) \otimes M \rightarrow M^{W} .
$$

Proof. The natural map $\mathcal{H}_{W ; \vec{T}}(X)^{W} \otimes M \rightarrow M^{W}$ is just the restriction of the map giving the action of the Hecke algebra on $M$. The local sections of $\mathcal{H}_{W ; \vec{T}}(X)^{W}$ have the form $\sum_{w \in W} w h$, where $h$ is a local section of $\mathcal{O}_{X}\left(D_{w_{0}}(-\vec{T})\right)$, and thus the image of the natural map consists of elements $\sum_{w \in W} w h m$, immediately giving the desired description. Note here that $\sum_{w \in W} w$ is indeed contained in $\mathcal{H}_{W ; \vec{T}}(X) \otimes \mathcal{O}_{X}\left(-D_{w_{0}}(-\vec{T})\right)$, so this is well-defined.

Taking the analogous result for $-\vec{T}$ and reexpressing everything in terms of the original Hecke algebra gives the following.

Proposition 4.63. The image of the natural map

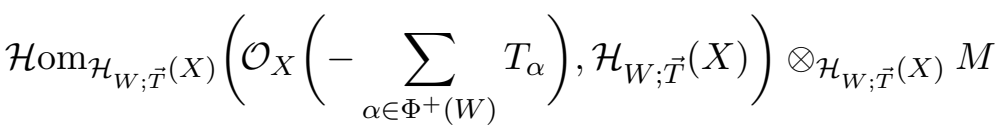

$$
\begin{aligned}
& \rightarrow \mathcal{H o m}_{\mathcal{H}_{W ; \vec{T}}(X)}\left(\mathcal{O}_{X}\left(-\sum_{\alpha \in \Phi^{+}(W)} T_{\alpha}\right), M\right)
\end{aligned}
$$


is naturally isomorphic to the image of the natural map

$$
m \mapsto \sum_{w \in W} w m: \mathcal{O}_{X}\left(D_{w_{0}}\right) \otimes M \rightarrow\left(\mathcal{O}_{X}\left(\sum_{\alpha \in \Phi^{+}(W)} T_{\alpha}\right) \otimes M\right)^{W}
$$

Using these, it is quite straightforward to obtain the desired strong flatness results (in those cases where the Hom sheaf itself is not strongly flat, that is): as images of maps of $S$-flat sheaves, the sheaves in question can only get smaller under specialization, so it suffices to show that the natural limits of the Bruhat subquotients are saturated. Each such subquotient reduces to the image of $\sum_{w \in W_{J}} w$ on a line bundle of the form $\mathcal{L}\left(D_{w_{J}}\right)$ where $\mathcal{L}$ is equivariant and descends in codimension 1 ; by the existence of symmetric idempotents when $\vec{T}=0$, we conclude that the image is just $\mathcal{L}^{W}$ independently of $\vec{T}$.

Proposition 4.64. There is a natural strongly flat family of bimodules which for $\vec{T}$ in general position is given by

$$
\mathcal{H}_{\operatorname{om}_{\mathcal{H}_{W ; \vec{T}}}(X)}\left(\operatorname{Ind}_{W_{J}}^{W ; \vec{T}} \mathcal{O}_{X}\left(-\sum_{\alpha \in \Phi^{+}\left(W_{J}\right)} T_{\alpha}\right), \operatorname{Ind}_{W_{I}}^{W ; \vec{T}} \mathcal{O}_{X}\right),
$$

and may be expressed as the intersection

$$
\mathcal{H}_{W, W_{I}, W_{J}}(X) \cap \mathcal{O}_{X}\left(\sum_{\alpha \in \Phi^{-}(W) \cup \Phi^{+}\left(W_{J}\right)} T_{\alpha}\right) \otimes \mathcal{H}_{W, W_{I}, W_{J}}(X) \otimes \mathcal{O}_{X}\left(-\sum_{\alpha \in \Phi^{-}(W) \backslash \Phi^{-}\left(W_{I}\right)} T_{\alpha}\right),
$$

compatibly with composition.

Proof. We may interpret $\operatorname{Ind}_{W_{I} ; \vec{T}}^{W} \mathcal{O}_{X}$ as $\mathcal{H}_{W, W_{I}, 1 ; \vec{T}}(X)$, and thus view it as a subsheaf of the parameter-free case $\mathcal{H}_{W, W_{I}, 1}(X)$. The operation $m \mapsto \sum_{w \in W_{J}} w m$ gives a well-defined (surjective) map

$$
\mathcal{O}_{X}\left(D_{w_{J}}\right) \mathcal{H}_{W, W_{I}, 1}(X) \rightarrow \mathcal{H}_{W, W_{I}, W_{J}}(X),
$$

and thus the (strongly flat) subsheaf discussed above of the Hom sheaf may itself be interpreted as a subsheaf of $\mathcal{H}_{W, W_{I}, W_{J}}(X)$.

Twisting gives the analogous subsheaf of

$$
\mathcal{H}_{\mathcal{H}_{W ;-\vec{T}}(X)}\left(\operatorname{Ind}_{W_{J}}^{W-\vec{T}} \mathcal{O}_{X}, \operatorname{Ind}_{W_{I}}^{W ;^{-} \vec{T}} \mathcal{O}_{X}\left(-\sum_{\alpha \in \Phi^{-}\left(W_{I}\right)} T_{\alpha}\right)\right) .
$$

Since this is itself a subsheaf of

$$
\mathcal{H}_{\mathrm{om}_{\mathcal{H}_{W}-\vec{T}}(X)}\left(\operatorname{Ind}_{W_{J}}^{W ;-\vec{T}} \mathcal{O}_{X}, \operatorname{Ind}_{W_{I}}^{W ;-\vec{T}} \mathcal{O}_{X}\right)=\mathcal{H}_{W, W_{I}, W_{J} ;-\vec{T}}(X),
$$

the other inclusion follows. Comparing Bruhat quotients gives the desired equality for $\vec{T}$ in general position.

Corollary 4.65. There is a natural strongly flat family of bimodules which for $\vec{T}$ in general position is given by

$$
\mathcal{H}_{\mathrm{om}_{\mathcal{H}_{W ; \vec{T}}}(X)}\left(\operatorname{Ind}_{W_{J} ; \vec{T}}^{W} \mathcal{O}_{X}, \operatorname{Ind}_{W_{I}}^{W ; \vec{T}} \mathcal{O}_{X}\left(-\sum_{\alpha \in \Phi^{+}\left(W_{I}\right)} T_{\alpha}\right)\right),
$$

and may be expressed as the intersection

$$
\mathcal{H}_{W, W_{I}, W_{J}}(X) \cap \mathcal{O}_{X}\left(\sum_{\alpha \in \Phi^{-}(W) \backslash \Phi^{-}\left(W_{J}\right)} T_{\alpha}\right) \otimes \mathcal{H}_{W, W_{I}, W_{J}}(X) \otimes \mathcal{O}_{X}\left(-\sum_{\alpha \in \Phi^{-}(W) \cup \Phi^{+}\left(W_{I}\right)} T_{\alpha}\right),
$$

compatibly with composition. 


\section{$5 \quad$ Infinite groups}

Most of our arguments above regarding the structure of the Hecke algebra boiled down to the combinatorics of (double) cosets in Coxeter groups and the associated Bruhat order. Indeed, virtually everything in the above discussion carries over immediately to the case of infinite Coxeter groups, with one glaring exception: the Hecke algebra was defined as a sheaf of algebras on the quotient $X / W$, and there is no such quotient scheme when $W$ is infinite!

Thus the primary (and to first approximation only) issue in generalizing the above construction is simply to determine what manner of object we will be constructing. Luckily, a suitable generalization of sheaves of algebras has already appeared in the literature on noncommutative geometry, namely the notion of a "sheaf algebra".

We recall the definition from [37, Section 2], generalizing an earlier definition of [2, Section 2]. We first need the notion of a sheaf bimodule: Let $X, Y$ be Noetherian $S$-schemes of finite type: An $\mathcal{O}_{S}$-central $\left(\mathcal{O}_{X}, \mathcal{O}_{Y}\right)$-bimodule is a quasicoherent $\mathcal{O}_{X \times{ }_{S} Y}$-module $M$ such that the support of any coherent subsheaf of $M$ is finite over both $X$ and $Y$ (relative to the projections). We will sometimes shorthand this by saying that $M$ is a sheaf bimodule on $X \times_{S} Y$. Note that if $X=\operatorname{Spec}\left(R_{X}\right), Y=\operatorname{Spec}\left(R_{Y}\right)$, then a sheaf bimodule on $X \times_{S} Y$ is an $\left(R_{X}, R_{Y}\right)$ bimodule such that $\Gamma\left(S ; \mathcal{O}_{S}\right)$ is central and such that any finitely generated sub-bimodule is finitely generated both as a left module and as a right module.

As with ordinary bimodules, there is a notion of tensor product for sheaf bimodules. If $M$ is a sheaf bimodule on $X \times_{S} Y$ and $N$ is a sheaf bimodule on $Y \times_{S} Z$, then we can construct a sheaf bimodule on $X \times_{S} Z$ by pulling back $M$ and $N$ to $X \times_{S} Y \times_{S} Z$, tensoring, and then taking the direct image to $X \times_{S} Z$ to obtain a sheaf $M \otimes_{Y} N$. Note that if $\Delta_{X / S}$ is the diagonal in $X \times_{S} X$, then $\mathcal{O}_{\Delta_{X / S}}$ is a sheaf bimodule on $X \times_{S} X$, and for any sheaf bimodule $M$ on $X \times_{S} Y$, there is a natural isomorphism $\mathcal{O}_{\Delta_{X / S}} \otimes_{X} M \cong M$. Furthermore, this tensor product operation is naturally associative and agrees with the usual tensor product when the schemes are affine, see [37].

The tensor product provides the category of $\left(\mathcal{O}_{X}, \mathcal{O}_{X}\right)$-bimodules with a natural monoidal structure, thus allowing one to define a sheaf algebra on $X / S$ to be a monoid object in that category; that is, a sheaf bimodule $A$ equipped with morphisms $\mathcal{O}_{\Delta_{X}} \rightarrow A$ and $A \otimes_{X} A \rightarrow A$ satisfying the obvious axioms. More generally, one may also consider sheaf categories, in which every object of the category has an associated scheme and the Hom sets are replaced by sheaf bimodules.

One difficulty in dealing with the above construction is that it is not always easy to work with local sections of the tensor product of sheaf bimodules. Indeed, since the tensor product is a direct image, we in general need to choose an affine open covering of $Y$ and look for compatible systems of elements of the corresponding naïve tensor products. It turns out that for coherent sheaf bimodules, there is a cleaner approach.

Proposition 5.1. Let $M$ be a sheaf bimodule on $X \times{ }_{S} Y$ and let $N$ be a sheaf bimodule on $Y \times{ }_{S} Z$. Let $\operatorname{Spec}(R) \cong V \subset Y$ be an affine open subset and let $U \subset X, W \subset Z$ be open subsets. If $M$ is coherent and the preimage of $V$ in the support of $M$ contains the preimage of $U$, or if $N$ is coherent and the preimage of $V$ in the support of $N$ contains the preimage of $W$, then there is a natural isomorphism

$$
\Gamma\left(U \times W ; M \otimes_{Y} N\right) \cong \Gamma(U \times V ; M) \otimes_{R} \Gamma(V \times W ; N) .
$$

Proof. By symmetry, we may suppose that the constraint on $M$ holds. The sections of a coherent sheaf bimodule on a product of open subsets depends only on the intersection of those open subsets on the support of the sheaf bimodule. It follows, therefore, that there is a natural isomorphism

$$
\Gamma\left(U \times V^{\prime} ; M\right) \cong \Gamma\left(U \times\left(V \cap V^{\prime}\right) ; M\right)
$$


for any open subset $V^{\prime}$. Computing the tensor product via an affine open covering of $Y$ containing $V$ gives a natural morphism

$$
\Gamma\left(U \times W ; M \otimes_{Y} N\right) \rightarrow \Gamma(U \times V ; M) \otimes_{R} \Gamma(V \times W ; N),
$$

and the compatibility conditions ensure that this is an isomorphism as required.

Remark. For coherent $M$, there is a maximal $U$ satisfying the hypothesis: take $X \backslash U$ to be the image of $X$ of the preimage of $Y \backslash V$, and observe that finiteness implies that this image is closed, so $U$ is open. For quasicoherent $M$, it is tempting to consider the intersection of the $U$ 's corresponding to the coherent subsheaves of $M$, but of course this will rarely be open. Of course, if it is open, then taking the limit tells us that the conclusion of the proposition continues to hold.

Of course, we would like to know that this incorporates the usual notion of a sheaf of algebras on the quotient.

Proposition 5.2. Let $f: X \rightarrow Y$ be a finite morphism of Noetherian $S$-schemes of finite type, and suppose that $\mathcal{A}$ is a quasicoherent sheaf of $\mathcal{O}_{Y}$-algebras on $Y$ equipped with an algebra morphism $f_{*} \mathcal{O}_{X} \rightarrow \mathcal{A}$. Then $\mathcal{A}$ induces a sheaf algebra $\mathcal{A}_{X}$ on $X / S$ such that for any open subsets $U, V \subset Y, \Gamma\left(f^{-1}(U) \times f^{-1}(V) ; \mathcal{A}_{X}\right) \cong \Gamma(U \cap V ; \mathcal{A})$.

Proof. As usual, we may assume that $S$ is affine. For any affine open subset $U \subset Y$, the sheaf-ofalgebras morphism $f_{*} \mathcal{O}_{X} \rightarrow \mathcal{A}$ induces an algebra morphism $\Gamma\left(U ; f_{*} \mathcal{O}_{X}\right) \rightarrow \Gamma(U ; \mathcal{A})$, and thus makes $\Gamma(U ; \mathcal{A})$ a bimodule over $\Gamma\left(U ; f_{*} \mathcal{O}_{X}\right) \cong \Gamma\left(f^{-1}(U) ; \mathcal{O}_{X}\right)$. More generally, if $U, V \subset Y$ are two affine open subsets, then we may use the morphisms $\Gamma\left(U ; f_{*} \mathcal{O}_{X}\right) \rightarrow \Gamma\left(U \cap V ; f_{*} \mathcal{O}_{X}\right)$ and $\Gamma\left(V ; f_{*} \mathcal{O}_{X}\right) \rightarrow \Gamma\left(U \cap V ; f_{*} \mathcal{O}_{X}\right)$ to make $\Gamma(U \cap V ; \mathcal{A})$ a $\left(\Gamma\left(f^{-1}(U) ; \mathcal{O}_{X}\right), \Gamma\left(f^{-1}(V) ; \mathcal{O}_{X}\right)\right)$ bimodule.

This in particular gives a family of $\left(\Gamma\left(f^{-1}\left(U_{i}\right) ; \mathcal{O}_{X}\right), \Gamma\left(f^{-1}\left(U_{j}\right) ; \mathcal{O}_{X}\right)\right)$-bimodules associated to any affine open covering of $Y$. Since the coefficient rings are commutative, we may reinterpret this as a $\Gamma\left(f^{-1}\left(U_{i}\right) ; \mathcal{O}_{X}\right) \otimes_{\mathcal{O}_{S}} \Gamma\left(f^{-1}\left(U_{j}\right) ; \mathcal{O}_{X}\right)$-module structure, and then observe that

$$
\Gamma\left(f^{-1}\left(U_{i}\right) ; \mathcal{O}_{X}\right) \otimes_{\mathcal{O}_{S}} \Gamma\left(f^{-1}\left(U_{j}\right) ; \mathcal{O}_{X}\right) \cong \Gamma\left(f^{-1}\left(U_{i}\right) \times_{S} f^{-1}\left(U_{j}\right) ; \mathcal{O}_{X \times_{S} X}\right) .
$$

The open subsets $f^{-1}\left(U_{i}\right) \times_{S} f^{-1}\left(U_{j}\right)$ cover $X \times_{S} X$, and their intersections have the same form, making it easy to see that one has natural and compatible restriction maps. It follows that these module structures glue together to give a sheaf on $X \times_{S} X$. Moreover, the sheaf is supported on the preimage in $X \times_{S} X$ of the diagonal in $Y \times_{S} Y$, and thus satisfies the requisite finiteness condition to be a sheaf bimodule.

It remains to see that this is a sheaf algebra. We note that for any open subset of $Y$, the pair of open subsets $\left(f^{-1}(U), f^{-1}(U)\right)$ satisfies the hypotheses of Proposition 5.1 for any coherent subsheaf of $\mathcal{A}_{X}$ on either side, from which it easily follows that the morphisms $f_{*} \mathcal{O}_{X} \rightarrow \mathcal{A}$ and $\mathcal{A} \otimes_{f_{*} \mathcal{O}_{X}} \mathcal{A} \rightarrow \mathcal{A}$ induce a sheaf algebra structure on $\mathcal{A}_{X}$.

There is a more general version of the approach to tensor products via open subsets that works for fairly general quasicoherent sheaf algebras and bimodules (including any case where the schemes are projective); this leads to some simpler alternate arguments and constructions below, but will not be strictly needed.

The construction of a sheaf algebra from a sheaf of algebras suggests defining an "invariant open subset" of a sheaf algebra, as an open subset $U$ such that the two preimages of $U$ in the support of any coherent subsheaf of the sheaf algebra agree. If $U$ is an affine open subset which is invariant for a given sheaf algebra $\mathcal{A}$, we immediately conclude that the sections on $U \times U$ of $\mathcal{A}$ form an algebra equipped with a morphism from $\Gamma\left(U ; \mathcal{O}_{U}\right)$. The difficulty, of course, is 
that a typical sheaf algebra will have no invariant affine opens. Luckily, the usual construction of a sheaf by gluing depends far more on the subsets being affine than that they be open. Define an affine localization of $X$ to be a nonempty affine scheme which is the directed limit of a (possibly infinite) family of open subschemes of $X$. As with affine opens, an affine localization is determined by its image in the underlying topological space of $X$, and if given a family of such subsets covering $X$ (in a locally finite way: every open subset of $X$ needs to be contained in a finite union of localizations), the corresponding family of morphisms will be faithfully flat. It then follows by fpqc descent that we may specify a sheaf (or morphisms of sheaves) using a covering by affine localizations in place of a covering by affine opens. One similarly finds that there is a well-behaved notion of "invariant" affine localization, and the restriction of a sheaf algebra to an invariant affine localization is an algebra.

There is still a difficulty here, in that there is no guarantee that there will always be a locally finite covering by invariant affine localizations. (For instance, the only invariant affine localization of the sheaf algebra $\bar{k}\left(\mathbb{P}^{1}\right)\left[\mathrm{PGL}_{2}(\bar{k})\right]$ on $\mathbb{P}_{\bar{k}}^{1}$ is the field $\bar{k}\left(\mathbb{P}^{1}\right)$ itself.) If there were an fpqc base change $S^{\prime} \rightarrow S$ such that the pullback to $\left(X \times_{S} S^{\prime}\right) \times_{S^{\prime}}\left(X \times_{S} S^{\prime}\right)$ was covered by invariant localizations, then we could work with those localizations to understand the algebra structure and then use a further application of fpqc descent to recover the morphisms on $X \times_{S} X$. Of course, rather than make two separate applications of fpqc descent, we could simply observe that $U_{i} \otimes_{S^{\prime}} U_{j} \rightarrow X \times_{S} X$ give an fpqc covering and do the descent directly. But this tells us that there was no need for the $U_{i}$ to cover $X \times_{S} S^{\prime}$; all we need is for them to cover $X$.

Given a scheme $S$, let $\hat{\mathbb{A}}_{S}^{1}$ denote the localization of $\mathbb{A}_{S}^{1}$ obtained as the limit of those open subschemes that are dense in every geometric fiber (equivalently, that contain the generic point of every fiber). Although the map $\hat{\mathbb{A}}_{S}^{1} \rightarrow \mathbb{A}_{S}^{1}$ is not an fpqc cover, the composition $\hat{\mathbb{A}}_{S}^{1} \rightarrow S$ is both fpqc and surjective, and thus an fpqc cover.

This construction is functorial in $S$ and if $S \rightarrow T$ is a finite morphism with $T$ Noetherian, then $\hat{\mathbb{A}}_{S}^{1} \cong \hat{\mathbb{A}}_{T}^{1} \times_{T} S$. Note, however, that this construction does not respect open embeddings, so the obvious way to associate a sheaf of algebras on $S$ to this construction does not always produce a quasicoherent sheaf.

We observe that if $X$ is projective over a Noetherian ring $R$, then $\hat{\mathbb{A}}_{X}^{1}$ is affine over $R$. Since the construction respects closed embeddings, it suffices to consider the case $X=\mathbb{P}_{R}^{n}$. In that case, we note that the section $\sum_{i} t^{i} x_{i}$ of the pullback of $\mathcal{O}_{X}(1)$ is invertible, and thus the trivial line bundle is very ample on $\hat{\mathbb{A}}_{X}^{1}$, making it affine. Since this holds for any embedding of $X$ in projective space, it follows that any very ample line bundle on $X$ becomes trivial on $\hat{\mathbb{A}}_{X}^{1}$, and thus (since very ample bundles generate the Picard group) that any line bundle on $X$ becomes trivial on $\hat{\mathbb{A}}_{X}^{1}$.

Proposition 5.3. Suppose $X$ and $Y$ are projective over the Noetherian affine scheme $S$, and let $M$ be a quasicoherent sheaf bimodule on $X \times_{S} Y$. Let $M^{\prime}$ be the base change of $M$ to $\hat{\mathbb{A}}_{S}^{1}$. Then the fiber products of $\hat{\mathbb{A}}_{X}^{1}$ and $\hat{\mathbb{A}}_{Y}^{1}$ with the support of any coherent subsheaf of $M^{\prime}$ are canonically isomorphic, and the affine localization $\hat{\mathbb{A}}_{X}^{1} \times_{\hat{\mathbb{A}}_{S}^{1}} \hat{\mathbb{A}}_{Y}^{1}$ of $X \times_{S} Y \times_{S} \hat{A}_{S}^{1}$ is an fpqc covering of $X \times_{S} Y$.

Proof. The only thing to observe is that the support of any coherent subsheaf of $M^{\prime}$ is contained in the base change of the support of a coherent subsheaf of $M$, and thus the claim reduces to the fact that the construction $\hat{\mathbb{A}}^{1}$ respects finite morphisms.

Remark. In particular, given bimodules on $X \times_{S} Y$ and $Y \times_{S} Z$, we can use the corresponding bimodules on $\hat{\mathbb{A}}_{X}^{1} \times_{\hat{\mathbb{A}}_{S}^{1}} \hat{\mathbb{A}}_{Y}^{1}$ and $\hat{\mathbb{A}}_{Y}^{1} \times_{\hat{\mathbb{A}}_{S}^{1}} \hat{\mathbb{A}}_{Z}^{1}$ to control the tensor product.

Thus when $X$ is projective, or more generally when $\hat{\mathbb{A}}_{X}^{1}$ is affine, we always have the option to replace the sheaf algebra with the actual algebra of global sections of $M$ on $\hat{\mathbb{A}}_{X}^{1} \times_{\hat{\mathbb{A}}_{S}^{1}} \hat{\mathbb{A}}_{X}^{1}$, and des- 
cent essentially reduces to checking that the algebra has a description which is independent of the auxiliary coordinate.

According to Proposition 5.2, the algebras $\mathcal{H}_{G}(X), \mathcal{H}_{W}(X), \mathcal{H}_{W ; \vec{T}}(X)$ from the previous section may all be interpreted as sheaf algebras on $X / S$, as can the twisted group algebras $k(X)[G]$, $\mathcal{O}_{X}[G]$. The latter are quite easy to generalize to the infinite case.

Proposition 5.4 ([37, Lemma 2.8]). Let $g$ be an automorphism of $X$. Then for any quasicoherent sheaf $M$ on $X,\left(1, g^{-1}\right)_{*} M$ is a sheaf bimodule, and for $h \in \operatorname{Aut}(X)$ and $N \in \operatorname{coh}(X)$, we have a natural isomorphism

$$
\left(1, g^{-1}\right)_{*} M \otimes_{X}\left(1, h^{-1}\right)_{*} N \rightarrow\left(1,(g h)^{-1}\right)_{*}\left(M \otimes{ }^{g} N\right) .
$$

Proof. Since $\left(1, g^{-1}\right)_{*} M$ is supported on the graph of an automorphism, the same applies to any coherent subsheaf, and thus it satisfies the requisite finiteness condition to be a sheaf bimodule.

Now, let $U$ be any affine open subset of $X$. Then $\left(U, g^{-1}(U)\right)$ satisfy the hypotheses of Proposition 5.1, and thus we have

$$
\begin{aligned}
\Gamma(U \times X ; & \left.\left(1, g^{-1}\right)_{*} M \otimes_{X}\left(1, h^{-1}\right)_{*} N\right) \\
& \cong \Gamma\left(U \times g^{-1}(U) ;\left(1, g^{-1}\right)_{*} M\right) \otimes_{\Gamma\left(g^{-1}(U) ; \mathcal{O}_{X}\right)} \Gamma\left(g^{-1}(U) \times X ;\left(1, h^{-1}\right)_{*} N\right) \\
& \cong \Gamma(U ; M) \otimes_{\Gamma\left(g^{-1}(U) ; \mathcal{O}_{X}\right)} \Gamma\left(g^{-1}(U) ; N\right),
\end{aligned}
$$

where $f \in \Gamma\left(g^{-1}(U) ; \mathcal{O}_{X}\right)$ acts on $\Gamma(U ; M)$ as multiplication by ${ }^{g} f$. With this action, there is a natural isomorphism

$$
\Gamma(U ; M) \otimes_{\Gamma\left(g^{-1}(U) ; \mathcal{O}_{X}\right)} \Gamma\left(g^{-1}(U) ; N\right) \cong \Gamma(U ; M) \otimes_{\Gamma\left(U ; \mathcal{O}_{X}\right)} \Gamma\left(U ;{ }^{g} N\right)
$$

given by $m \otimes n \mapsto m \otimes{ }^{g} n$, and thus the result follows.

Definition 5.5. Let $X / S$ be an Noetherian $S$-scheme of finite type with integral geometric fibers, and let $G$ be a group acting on $X$. Then the "twisted group sheaf algebra" $k(X)[G]$ is the sheaf

$$
\bigoplus_{g \in G}\left(1, g^{-1}\right)_{*} k(X)
$$

on $X \times X$ (with $k(X)$ denoting the sheaf of meromorphic functions on $X$ which are defined on the generic point of every geometric fiber of $X$ over $S$ ) with sheaf algebra structure induced by the natural morphisms

$$
\left(1, g^{-1}\right)_{*} k(X) \otimes_{X}\left(1, h^{-1}\right)_{*} k(X) \rightarrow\left(1,(g h)^{-1}\right)_{*} k(X)
$$

coming from the proposition.

Remark. We could also define this using an affine localization. The affine scheme $\hat{\mathbb{A}}_{X}^{1}$ constructed in Proposition 5.3 is functorial for $\operatorname{Aut}_{S}(X)$, and thus has an induced action of $G$. We may thus define a twisted group algebra $k(X) \otimes_{S} \mathcal{O}_{\hat{\mathbb{A}}_{X}^{1}}[G]$, and this has a natural associated descent datum. The only nontrivial thing to verify is the fact that cyclic bimodules are finitely generated on both sides, but this follows easily from the fact that $\hat{\mathbb{A}}_{X}^{1}$ is Noetherian: the bimodule $\mathcal{O}_{\hat{\mathbb{A}}_{X}^{1}} c_{g} g \mathcal{O}_{\hat{\mathbb{A}}_{X}^{1}}$ is cyclic on both sides, and any other cyclic bimodule is contained in a finite sum of such bimodules, so is finitely generated on both sides. 
We may similarly define $\mathcal{O}_{X}[G]$ to be the sheaf subalgebra $\bigoplus_{g \in G}\left(1, g^{-1}\right)_{*} \mathcal{O}_{X}$, which we readily verify to contain the image of the identity (a copy of $\mathcal{O}_{X}$ ) and be preserved by the multiplication map.

Moreover, we have the following.

Proposition 5.6. Let $g_{1}, \ldots, g_{n} ; h_{1}, \ldots, h_{m}$ be two finite subsets of $\operatorname{Aut}(X / S)$, and let $\mathcal{M}_{\left\{g_{1}, \ldots, g_{n}\right\}}, \mathcal{M}_{\left\{h_{1}, \ldots, h_{m}\right\}}, \quad \mathcal{M}_{\left\{g_{1} h_{1}, \ldots, g_{n} h_{m}\right\}}$ be interpreted as sheaf sub-bimodules of $k(X)[\operatorname{Aut}(X / S)]$. Then the multiplication on $k(X)[\operatorname{Aut}(X / S)]$ restricts to a morphism

$$
\mathcal{M}_{\left\{g_{1}, \ldots, g_{n}\right\}} \otimes \mathcal{M}_{\left\{h_{1}, \ldots, h_{m}\right\}} \rightarrow \mathcal{M}_{\left\{g_{1} h_{1}, \ldots, g_{n} h_{m}\right\}} .
$$

Proof. Given an open subset $V \subset X$, we may associate an open subset $U_{V}=\bigcap_{i} g_{i}(V)$, and we claim that there is an affine open covering $V_{i}$ of $X$ such that $U_{V_{i}}$ also covers $X$. Indeed, $x \in U_{V}$ iff $g_{1}^{-1}(x), \ldots, g_{n}^{-1}(X) \in V$, and thus if $V_{x}$ is an affine open neighborhood of this set of points, we have $x \in U_{V_{x}}$. It thus suffices to specify how the above morphism acts on local sections on sets of the form $U_{V} \times X$, for which we note

$$
\begin{aligned}
\Gamma\left(U_{V} \times X ;\right. & \left.\mathcal{M}_{\left\{g_{1}, \ldots, g_{n}\right\}} \otimes_{X} \mathcal{M}_{\left\{h_{1}, \ldots, h_{m}\right\}}\right) \\
& \cong \Gamma\left(U_{V} \times X ; \mathcal{M}_{\left\{g_{1}, \ldots, g_{n}\right\}}\right) \otimes_{\Gamma\left(V ; \mathcal{O}_{V}\right)} \Gamma\left(V \times X ; \mathcal{M}_{\left\{h_{1}, \ldots, h_{m}\right\}}\right) .
\end{aligned}
$$

But the result then follows immediately from the definition of $\mathcal{M}_{\left\{g_{1}, \ldots, g_{n}\right\}}$ as the space of operators preserving holomorphy.

Remark. When $X / S$ is projective, or more generally when we have nice affine localizations as constructed above, then we could define $\mathcal{M}_{\left\{g_{1}, \ldots, g_{n}\right\}}$ more simply as the subsheaf of $k(X)[\operatorname{Aut}(X / S)]$ supported on $\left\{g_{1}, \ldots, g_{n}\right\}$ and preserving the subring $\mathcal{O}_{\hat{\mathbb{A}}_{X}^{1}}$.

In particular, if $G$ is any subgroup of the group of automorphisms of $X / S$, we may define a sheaf algebra $\mathcal{H}_{G}^{+}(X)$ as the union in $k(X)[G]$ of the sheaves $\mathcal{M}_{\vec{g}}$ associated to finite subsets of $G$. More generally, we will wish to only allow poles on a proper (but $G$-invariant) subset of the reflection hypersurfaces, as otherwise in the affine Weyl group case we could acquire poles along the fibers where the " $q$ " parameter is torsion.

Suppose now that $X / S$ is a family of abelian varieties and that $(W, S)$ is a Coxeter group (of finite rank, but possibly infinite) equipped with an action on $X$ of coroot type. Then we define $\mathcal{H}_{W}(X)$ to be the sheaf subalgebra of $\mathcal{H}_{W}^{+}(X)$ consisting of operators which are holomorphic away from the reflection hypersurfaces corresponding to conjugates of the simple reflections. Clearly, this agrees with our previous notation, in that when $W$ is finite, $\mathcal{H}_{W}(X)$ is the sheaf algebra on $X$ associated to the sheaf of algebras on $X / W$ we previously denoted by $\mathcal{H}_{W}(X)$.

Since $W$ still has a Bruhat order, we may consider the subsheaf $\mathcal{H}_{W}(X)[I]$ for any order ideal $I \subset W$, and if the order ideal is finite, the result will be of the form $\mathcal{M}_{I}(X)$ and thus coherent. In fact, we have the following, by precisely the same argument as Lemma 4.16 and its corollaries.

Proposition 5.7. If $I$ is a finite order ideal in $W$ and $w \in I$ is a maximal element, then there is a short exact sequence

$$
0 \rightarrow \mathcal{H}_{W}(X)[I \backslash\{w\}] \rightarrow \mathcal{H}_{W}(X)[I] \rightarrow\left(1, w^{-1}\right)_{*} \mathcal{O}_{X}\left(D_{w}\right) \rightarrow 0
$$

of sheaf bimodules, where $D_{w}=\sum_{r \in R(W), r w<w}\left[X^{r}\right]$.

Corollary 5.8. For any reduced word $w=s_{1} \cdots s_{n}$, the multiplication map

$$
\mathcal{H}_{\left\langle s_{1}\right\rangle}(X) \otimes_{X} \cdots \otimes_{X} \mathcal{H}_{\left\langle s_{n}\right\rangle}(X) \rightarrow \mathcal{H}_{W}(X)[\leq w]
$$

is surjective. 
Corollary 5.9. The construction $\mathcal{H}_{W}(X)$ respects base change.

Since any finite subset of $W$ is contained in a finite order ideal, we also obtain the following.

Corollary 5.10. The sheaf algebra $\mathcal{H}_{W}(X)$ is the sheaf subalgebra of $k(X)[W]$ generated by the sheaf subalgebras $\mathcal{H}_{\langle s\rangle}(X)$ for $s \in S$.

Since the action of $W$ on $X$ is of coroot type, we still have a well-defined association of coroot morphisms to the roots of $X$, respecting positivity, and thus the notion of a system of parameters carries over.

Definition 5.11. The (untwisted) Hecke algebra $\mathcal{H}_{W: \vec{T}}(X)$ is the sheaf subalgebra of $\mathcal{H}_{W}(X)$ generated by the rank 1 sheaf algebras $\mathcal{H}_{\left\langle s_{i}\right\rangle, T_{i}}(X)$.

Lemma 4.29 and Corollary 4.31 again carry over immediately, as does the fact that this construction respects base change. However, the description of the adjoint and the description of Proposition 4.34 both founder on the fact that they involve a sum over all positive roots. To deal with this, we will need to generalize the construction further.

We first note that if we are given a $G$-equivariant gerbe $\mathcal{Z}$ (including, of course, the compatible explicit isomorphisms $\zeta_{g, h}: \mathcal{Z}_{g} \otimes{ }^{g} \mathcal{Z}_{h} \cong \mathcal{Z}_{g h}$ ), then as in the finite case, there is a corresponding crossed product algebra: take the sheaf bimodule $\bigoplus_{g}\left(1, g^{-1}\right)_{*} \mathcal{Z}_{g}$ with multiplication induced by $\zeta$. Of course, this also gives a twisted version of $k(X)[G]$ by replacing $\mathcal{Z}_{g}$ by its sheaf of meromorphic sections. Note that if $\mathcal{Z}_{g}$ and $\mathcal{Z}_{g}^{\prime}$ are meromorphically equivalent (i.e., there is a system of nonzero meromorphic maps between the line bundles which are compatible with the maps $\zeta$ ), then this induces an isomorphism between the corresponding meromorphic crossed product algebras. In particular, the meromorphic crossed product algebra associated to a given equivariant gerbe is isomorphic to the usual twisted group algebra iff there is a consistent family of meromorphic sections of $\mathcal{Z}_{g}$, iff the equivariant gerbe has the form $\mathcal{Z}_{g}=\mathcal{O}_{X}\left(Z_{g}\right)$, where $Z_{g}$ is a cocycle valued in Cartier divisors. (More generally, if one chooses arbitrary meromorphic sections, one obtains a meromorphic equivariant gerbe in which the line bundles are trivial but the maps $\zeta_{g, h}$ are only meromorphic, giving a class in $Z^{2}\left(G ; k(X)^{*}\right)$ and making the algebra a crossed product algebra in the usual sense. We will encounter an example of such a gerbe in Theorem 6.13 below.) We denote these sheaf algebras as $\mathcal{O}_{X}[G]_{\mathcal{Z}}$ and $k(X)[G]_{\mathcal{Z}}$ respectively.

In this generality, we cannot expect to have a well-defined analogue of $\mathcal{H}_{G}(X)$ without some additional data. For any line bundle $\mathcal{L}$, there is an induced sheaf algebra structure on $\mathcal{H}_{G}(X) \otimes_{X \times X} \mathcal{L} \otimes \mathcal{L}^{-1}$, which is sandwiched between $\mathcal{O}_{X}[G]_{\partial \mathcal{L}}$ and $k(X)[G]_{\partial \mathcal{L}}$, with $\partial \mathcal{L}$ denoting the coboundary gerbe $\mathcal{Z}_{g}=\mathcal{L} \otimes{ }^{g} \mathcal{L}^{-1}$. This equivariant gerbe is isomorphic to the trivial equivariant gerbe when $\mathcal{L}$ is $G$-equivariant (more precisely, such an isomorphism specifies a $G$ equivariant structure on $\mathcal{L}$ ), and this will in general give a different sheaf algebra sandwiched between $\mathcal{O}_{X}[G]$ and $k(X)[G]$. (Consider multiplying the equivariant structure by a character of $G$.)

We thus start with a more general construction. Given a subsheaf $\mathcal{A} \subset k(X)[G]_{\mathcal{Z}}$ and a subset $S \subset G$, let $\left.\mathcal{A}\right|_{S}$ denote the subsheaf of operators supported on $S$ (which is a subalgebra if $S$ is a subgroup). An order in $k(X)[G]_{\mathcal{Z}}$ is defined to be a torsion-free sheaf algebra $\mathcal{A}$ with generic fiber $k(X)[G]_{\mathcal{Z}}$ such that $\left.\mathcal{A}\right|_{S}$ is coherent for every finite subset $S \subset G$. (Note that if $G$ is finite, then $k(X)[G]_{\mathcal{Z}}$ may be viewed as a central simple algebra over $k(X / G)$, and this is precisely the usual notion of order.) In particular, $\mathcal{O}_{X}[G]_{\mathcal{Z}}$ is an order in $k(X)[G]_{\mathcal{Z}}$, and $\mathcal{H}_{G}(X)$ is an order in $k(X)[G]$.

We are interested in a particular subclass of orders, namely those which are left reflexive, in that each sheaf $\left.\mathcal{A}\right|_{S}$ is reflexive as a left $\mathcal{O}_{X}$-module. The order $\mathcal{O}_{X}[G]_{\mathcal{Z}}$ is clearly left reflexive, and since an element of $k(X)[G]$ is a (left) local section of $\mathcal{H}_{G}(X)$ iff it is a local section over every codimension 1 local ring, $\mathcal{H}_{G}(X)$ is also left reflexive. (Note that when $G$ is finite but $X \rightarrow X / G$ is not flat, $\mathcal{H}_{G}(X)$ is left reflexive as an $\mathcal{O}_{X}$ module, but not as an $\mathcal{O}_{X / G}$-module.) This suggests that we should consider left reflexive orders more generally. 
Proposition 5.12. Let $\mathcal{A}$ be a left reflexive order in $k(X)[G]_{\mathcal{Z}}$ containing $\mathcal{O}_{X}[G]_{\mathcal{Z}}$. For any open subset $U \subset X$, an element $D \in k(X)[G]_{\mathcal{Z}}$ is in $\Gamma(U \times X ; \mathcal{A})$ iff for every codimension 1 point $x \in U$, with inertia group $I_{x}, D \in \mathcal{O}_{X, x} \otimes_{\mathcal{O}_{X}}\left(\left.\mathcal{A}\right|_{I_{x}} \mathcal{O}_{X}[G]_{\mathcal{Z}}\right)$.

Proof. Since $\mathcal{A}$ is left reflexive, we certainly have that $D$ is a local section iff $D \in \mathcal{O}_{X, x} \otimes_{\mathcal{O}_{X}} \mathcal{A}$ for all $x \in U$ of codimension 1 , as this is true for any reflexive sheaf on $X$, and $\mathcal{A}$ inherits it from its restrictions to finite subsets. We thus need to understand the modules $\left.\mathcal{O}_{X, x} \otimes \mathcal{O}_{X} \mathcal{A}\right|_{S}$ for finite $S$. But then we may proceed as in the proof of Lemmas 4.7 and 4.6 to deduce that

$$
\left.\mathcal{O}_{X, x} \otimes_{\mathcal{O}_{X}} \mathcal{A}\right|_{S}=\left.\bigoplus_{g \in I_{x} \backslash G} \mathcal{O}_{X, x} \otimes \mathcal{A}\right|_{I_{x} g \cap S}
$$

and thus reduce to the case $S \subset I_{x} g$. Since $\left.\left.\mathcal{A}\right|_{I_{x}} \mathcal{Z}_{g} g \subset \mathcal{A}\right|_{I_{x} g}$ and $\left.\left.\mathcal{A}\right|_{I_{x}} \subset \mathcal{A}\right|_{I_{x} g} \mathcal{Z}_{g^{-1}} g^{-1}$, we conclude that $\left.\mathcal{A}\right|_{I_{x} g}=\left.\mathcal{A}\right|_{I_{x}} \mathcal{Z}_{g} g$, giving the desired result.

Proposition 5.13. Let $X$ be a normal integral scheme equipped with an action of the group $G$ and an equivariant gerbe $\mathcal{Z}$, and for each codimension 1 point $x \in X$, let $\mathcal{A}_{x}$ be an subalgebra of $k(x)\left[I_{x}\right]_{\mathcal{Z}}$ containing $\mathcal{O}_{X, x}\left[I_{x}\right]_{\mathcal{Z}}$ and such that $\left.\mathcal{A}_{x}\right|_{S}$ is a free left $\mathcal{O}_{X, x}$-module for every finite subset $S$. Suppose moreover that for any $g \in G$, we have $\mathcal{A}_{g x}=\mathcal{Z}_{g} g \mathcal{A}_{x} \mathcal{Z}_{g^{-1}} g^{-1}$. Then there is a unique left reflexive order $\mathcal{A}$ in $k(X)[G]_{\mathcal{Z}}$ such that $\left.\mathcal{O}_{X, x} \otimes_{\mathcal{O}_{X}} \mathcal{A}\right|_{I_{x}}=\mathcal{A}_{x}$ for all $x$.

Proof. For each finite $S \subset G$, we certainly obtain a well-defined left reflexive sheaf bimodule consisting of operators $\sum_{g \in S} c_{g} g$ which are in $\mathcal{A}_{x} \mathcal{O}_{X}[G]_{\mathcal{Z}}$ for every $x$; the point is that just as in the case of holomorphy-preserving operators, there are only finitely many $x$ such that $S$ meets some $I_{x} g$ in more than one element. By the previous proposition, any algebra as described must contain these sheaves, so it remains only to show that these sheaf bimodules are compatible under multiplication. That is, if $D_{1}$ is a section on $U \times X$ and $D_{2}$ is a section on $X \times V$, then $D_{1} D_{2}$ is a section on $U \times V$. We thus need to check that $D_{1} D_{2}$ is in $\mathcal{A}_{x} \mathcal{O}_{X}[G]_{\mathcal{Z}}$ for every codimension 1 point $x \in U$. Since this must hold for all $U$, we may as well take the limit and thus take $D_{1}$ to be an element of the left stalk at $x$. Since this splits as a direct sum, we may further suppose that $D_{1}$ is supported on a single coset of the inertia group, so that $D_{1} \in \mathcal{A}_{x} \mathcal{Z}_{g} g$. This is equivalent to $D_{1} \in \mathcal{Z}_{g} g \mathcal{A}_{g^{-1} x}$, and we find

$$
\mathcal{A}_{x} \mathcal{Z}_{g} g D_{2} \subset \mathcal{A}_{x} \mathcal{O}_{X}[G]_{\mathcal{Z}} \otimes \mathcal{O}_{V}
$$

iff

$$
\mathcal{A}_{g^{-1}{ }_{x}} D_{2} \subset \mathcal{A}_{g^{-1}{ }_{x}} \mathcal{O}_{X}[G]_{\mathcal{Z}} \otimes \mathcal{O}_{V}
$$

Since $D_{2}$ is a section of the sheaf, we have

$$
D_{2} \in \mathcal{A}_{y} \mathcal{O}_{X}[G]_{\mathcal{Z}} \otimes \mathcal{O}_{V}
$$

for every codimension 1 point $y \in X$, and thus for $y=g^{-1} x$, and the claim follows from the fact that $\mathcal{A}_{g^{-1} x}$ is an algebra.

We thus see that the construction of a reasonable analogue of $\mathcal{H}_{G}(X)$ reduces to constructing an analogue for each inertia group, subject to the compatibility conditions under conjugation. There are two approaches we might take to this. The first is that if we are given a trivialization of the gerbe (or, more precisely, its restriction to $I_{x}$ ) along the local ring at $x$, then this lets us pull $\mathcal{H}_{I_{x}}(X)$ back to an algebra containing $\mathcal{O}_{X, x}\left[I_{x}\right]_{\mathcal{Z}}$ which we may use as an ingredient in the above construction. Note that when $I_{x}$ is finite, we do not actually require $I_{x}$ to be a trivialization of the gerbe for this to work; it merely needs to be an approximate trivialization, 
since what we are really determining is the quotient $\mathcal{O}_{X, x}$-module $\mathcal{O}_{X, x} \otimes \mathcal{H}_{I_{x}}(X) / \mathcal{O}_{X, x}\left[I_{x}\right]_{\mathcal{Z}}$. Equivalently, we may ask for a trivialization over the complete local ring.

A second approach is to simply ask for an order of approximately the same "shape". This is particularly feasible in the case of order 2 reflections. Although the result is the same in most cases, this will be particularly useful for us, as it will be easy to extend to more general base schemes. With this in mind, let $X / S$ be a normal scheme with an action of an involution $s$ such that the fixed subscheme $X^{s}$ is an irreducible hypersurface, and let $\mathcal{Z}_{s}, \zeta_{s}$ be a gerbe, so that $\zeta_{s}: \mathcal{Z}_{s} \otimes{ }^{s} \mathcal{Z}_{s} \rightarrow \mathcal{O}_{X}$ is an isomorphism satisfying $\zeta_{s}={ }^{s} \zeta_{s}$. An obvious "shape" to take for a larger algebra would be to take operators $c_{1}+c_{s} s$ such that $c_{1} \in \mathcal{O}_{X}\left(\left[X^{s}\right]\right), c_{s} \in \mathcal{Z}_{s}\left(\left[X^{s}\right]\right)$, and $c_{1}+h c_{s} \in \mathcal{O}_{X, X^{s}}$ for some rational map $h: \mathcal{Z}_{s} \rightarrow \mathcal{O}_{X}$ which is holomorphic along $X^{s}$. We may then readily verify that the result is closed under multiplication iff $\zeta_{s}-h^{s} h$ vanishes along $2\left[X^{s}\right]$. Note that if $h-h^{\prime}$ vanishes on $X^{s}$, then replacing $h$ by $h^{\prime}$ gives the same algebra; the consistency condition is unchanged since $h^{s} h-h^{\prime s} h^{\prime}$ vanishes along $2\left[X^{s}\right]$. (Note that we may view $h^{s} h$ as the pullback of the norm of $h$ down to $X /\langle s\rangle$, and this interpretation induces a natural norm from $\operatorname{Hom}\left(\mathcal{Z}_{s}, \mathcal{O}_{\left[X^{s}\right]}\right)$ to $\operatorname{Hom}\left(\mathcal{Z}_{s} \otimes{ }^{s} \mathcal{Z}_{s}, \mathcal{O}_{2\left[X^{s}\right]}\right)$.) This is very nearly the same as asking for a trivialization over the complete local ring; indeed, the two notions disagree only when the residue field is an inseparable extension of the residue field of the $s$-invariant subring.

In particular, given a line bundle $\mathcal{L}$ on $X$, we have a coboundary gerbe $\mathcal{Z}_{s}=\mathcal{L} \otimes{ }^{s} \mathcal{L}^{-1}$, and there is a natural choice of $h$, namely $h=\left.{ }^{s} f f^{-1}\right|_{\left[X^{s}\right]}$ for some (any) meromorphic section $f$ of $\mathcal{L}$ which is holomorphic and not identically 0 along $X^{s}$. This, of course, simply corresponds to the usual notion of twisting by a line bundle. This coboundary operation is functorial in a particularly strong sense: not only is it functorial, but the functor takes any automorphism to the identity. Thus if instead of a line bundle on $X$ we are given a line bundle $\mathcal{L}_{T}$ on the base change $X_{T}$ to some fppf cover, then all we need for the coboundary to descend to $S$ is for the two pullbacks of $\mathcal{L}_{T}$ to $T \times{ }_{S} T$ to be isomorphic (that is, they need not be compatibly isomorphic!). Indeed, if $\mathcal{L}_{T}$ does not descend, then the obstruction is given by an automorphism of a pullback to $T \times{ }_{S} \times T \times{ }_{S} T$, and the coboundary functor turns this into the identity. In particular, if $X / S$ is projective, any section of the relative Picard scheme gives rise to a well-defined coboundary.

More generally, given $X / S$ with an action of an involution $s$ such that $X^{s}$ is nonempty and everywhere of codimension 1, define a "twisting datum" to be an equivariant gerbe equipped with a morphism $h_{s}: \mathcal{Z}_{s} \rightarrow \mathcal{O}_{\left[X^{s}\right]}$ of norm $\zeta_{s}$. Note that if $X / S$ is proper, then $\zeta_{s}$ is the pullback of a function on $S$, so is determined by its restriction to $2\left[X^{s}\right]$, and thus by $h_{s}$, which must merely satisfy the requirement that its norm be invertible and constant. For each fppf $T / S$, let $\operatorname{Tw}^{0}(X / S)(T)$ be the group of twisting data with $\mathcal{Z}_{s}=\mathcal{O}_{X}$; when $X$ is proper, this is the set of global sections of $\mathcal{O}_{\left[X^{s}\right] \times_{S} T}$ with norm in $\mathcal{O}_{T}^{*}$, and thus $\mathrm{Tw}^{0}(X / S)$ is represented by a group scheme. There is a natural morphism to this group scheme from the sheaf of groups consisting of pairs $(\mathcal{L}, \psi)$ with $\psi:{ }^{s} \mathcal{L} \cong \mathcal{L}$ : take the coboundary twisting datum and use $\psi$ to make the line bundle trivial. When $X / S$ is projective, this sheaf of groups is itself representable; it is a $\mathbb{G}_{m}$-bundle over the $s$-invariant subscheme of the Picard scheme of $X$. Moreover, there is a natural homomorphism from $\operatorname{Pic}(X /\langle s\rangle)$ to this scheme given by pulling back and letting $\psi$ be the natural equivariant structure.

Lemma 5.14. Let $C / S$ be a hyperelliptic curve of genus 1 . Then the above morphisms give rise to a short exact sequence $0 \rightarrow \operatorname{Pic}(C /\langle s\rangle) \rightarrow \mathbb{G}_{m}$. $\operatorname{Pic}(C)^{\langle s\rangle} \rightarrow \mathrm{Tw}^{0}(C) \rightarrow 0$ of group schemes.

Proof. Since $\operatorname{Pic}(C /\langle s\rangle) \cong \mathbb{Z}$ (the free group generated by the isomorphism class $\mathcal{O}(1)$ ) and the first map simply doubles degree, we find that it is indeed injective. A pair $(\mathcal{L}, \psi)$ induces the gerbe $\left(\mathcal{O}_{C}, \psi^{s} \psi\right)$, so the gerbe is trivial iff $\psi$ makes $\mathcal{L}$ equivariant. We then find that $h$ is given by the restriction of the equivariant structure to $C^{s}$, and thus is trivial iff $\mathcal{L}$ descends to the quotient; thus the sequence is exact in the middle. 
It remains to show that the second morphism is surjective. This is essentially a statement about fppf sheaves and thus we may feel free to base change so that $C / S$ is elliptic. In that case, both groups map to $\mathbb{G}_{m}$ by taking restrictions to the identity, and it will suffice to show that the remaining factors are isomorphic. We readily verify that the subgroup of $\operatorname{Tw}^{0}(E)$ such that $h(0)=1$ is a finite group scheme of order 8 in every fiber; indeed, it may be identified with the complete intersection of 3 quadrics in a suitable $\mathbb{P}^{3}$. Similarly, the relevant quotient of $\operatorname{Pic}(E)^{\langle s\rangle}$ may be identified with the disjoint union of two copies of $E[2]$ (for $C$, this is $\operatorname{Pic}^{0}(C)[2]$ and the torsor of Weierstrass points), so is also a finite flat group scheme of order 8. The image of $\mathcal{L}_{1}$ is (by definition) the quadratic function $\mathfrak{q} \in \mu_{2}(E[2])$ considered above, which in characteristic not 2 is 1 at 0 and -1 at the nontrivial 2 -torsion points. The action of $E[2]$ by translation induces an action on both groups, and the homomorphism is equivariant. In particular, we may compute the image of $x \in E[2](S)$ in $\operatorname{Tw}^{0}(E)$ as the image of $x^{*} \mathcal{L}_{1} \otimes \mathcal{L}_{1}^{-1}$. Modulo overall scalars, this is $\mathfrak{q}(z+x) \mathfrak{q}(z)^{-1}$; applying the splitting gives $\mathfrak{q}(z+x) \mathfrak{q}(z)^{-1} \mathfrak{q}(x)^{-1}$. Since this is precisely the Weil pairing of $z$ and $x$, we conclude that the restriction of the coboundary morphism to $E[2]$ is the Weil pairing, and in particular is injective. Since the image of any element of this index 2 subgroup scheme is a homomorphism and $\mathfrak{q}$ is not a homomorphism, we conclude that the morphism on $\mathrm{Pic}^{0}(E)[2] \uplus \operatorname{Pic}^{1}(E)^{\langle s\rangle}$ is injective, and thus by degree considerations is surjective.

Corollary 5.15. Any twisting datum for the action of $s$ on $C$ is isomorphic to a coboundary.

Proof. Since the line bundle $\mathcal{Z}_{s}$ has norm $\mathcal{O}_{C / s}$, it must have degree 0 , and since multiplication by 2 is surjective on the group scheme $\mathrm{Pic}^{0}(C)$, there is an fppf covering $T \rightarrow S$ and a line bundle $\mathcal{L}$ of degree 0 on $C_{T}$ such that $\mathcal{L} \otimes{ }^{s} \mathcal{L}^{-1} \cong \mathcal{Z}_{s}$. If we choose such an isomorphism, then the coboundary induces a twisting datum with line bundle $\mathcal{Z}_{s}$, and thus we may divide our original twisting datum by this new twisting datum to obtain a datum with trivial line bundle. By the lemma, this is the image of $\left(\mathcal{L}^{\prime}, \psi\right)$, where $\mathcal{L}^{\prime}$ is an isomorphism class of line bundles of degree 0 or 1 and $\psi: \mathcal{L}^{\prime} \cong{ }^{s} \mathcal{L}^{\prime}$. We thus conclude that the base change of the original twisting datum is isomorphic to the coboundary of $\mathcal{L} \otimes \mathcal{L}^{\prime}$. The two pullbacks of this bundle to $T \times{ }_{S} T$ have the same coboundary, so must differ by a pullback from $\mathcal{O}_{C /\langle s\rangle}$, which by degree considerations must be trivial. Thus $\mathcal{L} \otimes \mathcal{L}^{\prime}$ corresponds to an $S$-point of the relative Picard scheme of $C$, and the twisting datum is the coboundary of this point.

We may extend the notion of twisting datum to more general groups by assigning a twisting datum to each order 2 subgroup that fixes some reflection hypersurface, and insist on the appropriate compatibility relations. Again, any point of the relative Picard scheme has a well-defined coboundary.

Proposition 5.16. Let $W$ be a finite Weyl group and let $X / S$ be an abelian torsor on which $W$ acts by reflections. If the root kernel of $X$ is trivial, then any twisting datum on $X$ is isomorphic to a coboundary.

Proof. Let $T / S$ be an fppf cover over which $X$ has a $W$-invariant section. The induced twisting datum on each rank 1 parabolic subgroup is the coboundary of a point in the Picard scheme, but since $X_{T}$ has a section, it is in fact the coboundary of a line bundle on $X_{T}$. We may then apply Proposition 3.13 to express the induced class in $Z^{1}\left(W ; \operatorname{Pic}\left(X_{T}\right)\right)$ as a coboundary. Moreover, we may use the invariant section (which is contained in every $\left[X^{s}\right]$ ) to rigidify the various isomorphisms, and thus express the base changed twisting datum as the product of this coboundary and a twisting datum with trivial underlying gerbe.

Choose representatives among the simple reflections of the conjugacy classes of reflections, and observe that for each such $s_{i}$, there is a unique bundle $\mathcal{L}_{i}$ on $E_{i}$ of degree 0 or 1 such that the restriction of the latter twisting datum on $s_{i}$ is the coboundary of $\pi_{i}^{*} \mathcal{L}_{i}$. Tensoring 
the conjugates of those bundles gives a $W$-invariant (but not equivariant) line bundle with the desired twisting datum, and thus expresses the base change of the original twisting datum as the coboundary of a line bundle $\mathcal{L}_{T}$.

Since the two pullbacks of $\mathcal{L}_{T}$ to $T \times_{S} T$ have the same coboundary, they differ by an equivariant bundle on $X_{T}$ that descends in codimension 1 . Since the polarization of a line bundle is locally constant, we may arrange for the ratio to have trivial polarization, so be a point of $\operatorname{Pic}^{0}(X)\left(T \times_{S} T\right) \cong \prod_{i} E_{i}\left(T \times_{S} T\right)$. The restriction to $s_{i}$ of the coboundary of such a point is essentially just the coboundary of the corresponding point of $E_{i}\left(T \times{ }_{S} T\right)$; since it must be trivial, we conclude that the two pullbacks of $\mathcal{L}_{T}$ are in fact isomorphic, and thus that $\mathcal{L}$ descends to a point of the relative Picard scheme of $X / S$.

Remark. This can fail if the root kernel is nontrivial, even when $X$ has a section. However, there is a flat finite cover $T \rightarrow S$ over which $X$ can be expressed as the quotient of a torsor with trivial root kernel, so that we may describe a twisting datum on $X$ as the coboundary of a line bundle on $X_{T}$, subject to appropriate descent conditions.

More generally, if $W$ is a Coxeter group and $X / S$ is an abelian torsor with an action of $W$ of coroot type, then an assignment of twisting data on the rank 1 parabolic subgroups extends to at most one twisting datum for $W$. Indeed, we may extend the collection of pairs $\left(\mathcal{Z}_{s_{i}}, \zeta_{i}\right)$ to an equivariant gerbe by choosing a reduced word for each $w \in W$ and defining

$$
\mathcal{Z}_{w}:=\mathcal{Z}_{s_{1}} \otimes{ }^{s_{1}} \mathcal{Z}_{s_{2}} \otimes{ }^{s_{1} s_{2}} \mathcal{Z}_{s_{3}} \otimes \cdots \otimes{ }^{s_{1} \cdots s_{m-1}} \mathcal{Z}_{s_{m}} .
$$

If all we had was the equivariant gerbe structure, we would also need to specify isomorphisms corresponding to the different braid relations, which would themselves need to satisfy compatibility relations (coming from finite parabolic subgroups of rank $\leq 3$ ). ${ }^{3}$ Luckily, each braid relation may be restated as a conjugacy relation between simple reflections, and the isomorphism corresponding to the braid relation appears linearly in the corresponding consistency condition on the twisting data. For any rank $\leq 3$ finite parabolic subgroup, the different reflection hypersurfaces have a nonempty common intersection, and thus the further compatbility conditions of the gerbe will be automatically satisfied. Thus a collection of rank 1 twisting data extends to a full twisting datum iff it extends for every finite rank 2 subgroup.

Let $\gamma$ denote such a twisting datum, and write $k(X)[W]_{\gamma}$ for $k(X)[W]_{\mathcal{Z}_{\gamma}}$, and let $\mathcal{H}_{W ; \vec{T} ; \gamma}(X)$ denote the sheaf subalgebra generated by the rank 1 algebras $\mathcal{L}_{i} \otimes \mathcal{H}_{\left\langle s_{i}\right\rangle, T_{i}}(X) \otimes \mathcal{L}_{i}^{-1}$, where $\mathcal{L}_{i}$ is any line bundle with coboundary $\left.\gamma\right|_{s_{i}}$. Then the usual arguments carry over from the finite case to give the following.

Proposition 5.17. If $I$ is a finite order ideal in $W$ and $w \in I$ is a maximal element, then there is a short exact sequence

$$
0 \rightarrow \mathcal{H}_{W ; \vec{T} ; \gamma}(X)[I \backslash\{w\}] \rightarrow \mathcal{H}_{W ; \vec{T} ; \gamma}(X)[I] \rightarrow\left(1, w^{-1}\right)_{*}\left(\mathcal{Z}_{\gamma, w} \otimes \mathcal{O}_{X}\left(D_{w}(\vec{T})\right)\right) \rightarrow 0
$$

of sheaf bimodules.

Corollary 5.18. The construction of the sheaf algebra $\mathcal{H}_{W ; \vec{T} ; \gamma}(X)$ respects base change.

Corollary 5.19. Let $\vec{T}$ be a system of parameters such that every $T_{\alpha}$ is transverse to every reflection hypersurface. Then $\mathcal{H}_{W ; \vec{T} ; \gamma}(X)$ may be identified with the sheaf subalgebra of $\mathcal{H}_{W ; \gamma}(X)$ consisting locally of operators $\sum_{w} c_{w} w$ such that for every $w, c_{w}$ vanishes on the divisor $\sum_{\alpha \in \Phi^{+}(W) \cap w \Phi^{-}(W)} T_{\alpha}$.

\footnotetext{
${ }^{3}$ In general, one can define a $G$-equivariant gerbe by giving a line bundle for each generator of $G$, a morphism for each relation, subject to a consistency condition for each 3-cell of the classifying space $B G$. For Coxeter groups, there is a model of $B W$ with $k$-cells corresponding to multisets of $k$ simple roots such that the corresponding parabolic subgroup is finite, and thus the 3-cells come from finite parabolic subgroups of rank $\leq 3$.
} 
Corollary 5.20. Let $w \in W$ be given by the reduced word $w=s_{1} \cdots s_{l}$. Then the multiplication map $\mathcal{H}_{\left\langle s_{1}\right\rangle, \vec{T} ; \gamma}(X) \otimes_{X} \cdots \otimes_{X} \mathcal{H}_{\left\langle s_{l}\right\rangle, \vec{T} ; \gamma}(X) \rightarrow \mathcal{H}_{W ; \vec{T} ; \gamma}(X)[\leq w]$ is surjective. Moreover, any product of rank 1 subalgebras is equal to some Bruhat interval.

Suppose that $D_{1}, \ldots, D_{n}$ are Cartier divisors such that $Z_{s_{i}}=D_{i}-{ }^{s_{i}} D_{i}$ extends to a cocycle valued in Cartier divisors, and consider the case that $\gamma_{i}=\partial \mathcal{O}_{X}\left(D_{i}\right)$. Since the action of $W$ on the group of Cartier divisors is a permutation module, its restriction to any finite subgroup is induced from a trivial module, so has trivial $H^{1}$. In particular $\left.Z\right|_{\left\langle s_{i}, s_{j}\right\rangle}$ is a coboundary of some $D_{i j}$ for any finite rank 2 parabolic subgroup. If $D_{i}-D_{i j}$ has even valuation along any (separable) component of $\left[X^{s_{i}}\right]$, and similarly for $D_{j}-D_{i j}$, then $\gamma_{i}=\partial \mathcal{O}_{X}\left(D_{i j}\right)$ and similarly for $\gamma_{j}$, and thus we have a compatible extension of twisting data. Note that since $D_{i j}$ is only determined up to $\left\langle s_{i}, s_{j}\right\rangle$-invariant divisors, we can change its parity along each orbit of $\left\langle s_{i}, s_{j}\right\rangle$ reflection hypersurfaces independently, and thus if $s_{i}$ and $s_{j}$ are not conjugate, this condition can always be satisfied, and otherwise reduces to a simple parity constraint.

Given any other twisting datum $\gamma$, let $\gamma(\vec{D})$ denote the twisting datum obtained by tensoring with the above twisting datum. Since $Z_{s_{i}}$ extends to a cocycle valued in Cartier divisors, the resulting equivariant gerbe comes with a natural meromorphic equivalence to the original equivariant gerbe, and thus we have an induced isomorphism $k(X)[W]_{\gamma} \cong k(X)[W]_{\gamma(\vec{D})}$ for any $\gamma$, and may in this way view $\mathcal{H}_{W ; \vec{T} ; \gamma(\vec{D})}$ as a subalgebra of $k(X)[W]_{\gamma}$.

To understand such isomorphisms more generally, we will need to understand cocycles valued in Cartier divisors. The fact that $\operatorname{Hom}(W, \mathbb{Z})=0$ implies that any coinduced module for $W$ has trivial $H^{1}$. Since Cartier divisors are a sum of induced modules, there can be (and are) cocycles valued in Cartier divisors which are not coboundaries. However, since the induced modules are contained in the corresponding coinduced modules, we can always express such a cocycle as a coboundary in the larger module (of integer-valued functions on the set of irreducible Cartier divisors). Note that since the typical element of a coinduced module will not have coboundary in the induced submodule, we need to add the condition that any element of $w$ only changes finitely many values of the function; naturally, it suffices to verify the condition for the simple reflections.

For instance, if we interpret $\sum_{\alpha \in \Phi^{+}(W)} T_{\alpha}$ as giving an integer-valued function on irreducible Cartier divisors (i.e., the sum over $\alpha \in \Phi^{+}(W)$ of the valuation of $T_{\alpha}$ along the given divisor), then any element of $W$ only changes finitely many values of the function, and thus we obtain a well-defined coboundary $Z_{w}=\sum_{\alpha \in \Phi^{+}(W) \cap w \Phi^{-}(W)}\left(T_{\alpha}-T_{-\alpha}\right)$.

We may also use such formal sums to define (meromorphically trivial) twisting data; if $\Gamma$ is an integer-valued function on irreducible Cartier divisors such that $\Gamma-{ }^{s_{i}} \Gamma$ has finite support, then we may obtain a divisor $D_{i}$ with the same coboundary on $\left\langle s_{i}\right\rangle$ by restricting $\Gamma$ to the union of the support of $\Gamma-{ }^{s_{i}} \Gamma$ and the components of the reflection hyperplanes. Similarly, if $\left\langle s_{i}, s_{j}\right\rangle$ is finite, then we may obtain a divisor $D_{i j}$ by restricting $\Gamma$ to the union of the supports of $\Gamma-{ }^{w} \Gamma$ for $w \in\left\langle s_{i}, s_{j}\right\rangle$, and find that $D_{i j}-D_{i}$ and $D_{i j}-D_{j}$ are pullbacks, so that $\gamma_{i}=\partial \mathcal{O}_{X}\left(D_{i}\right)$ gives a well-defined twisting datum. We denote the twist of some other $\gamma$ by this meromorphically trivial datum by $\gamma(\Gamma)$. (More precisely, a twisting datum is determined by $\Gamma$ along with a choice of representation of each $\Gamma-{ }^{s_{i}} \Gamma$ as a coboundary; the above convention can behave badly in families, but there is always a consistent way to take a limit of the choices of representations as coboundaries in rank 1.)

We then introduce the notation

$$
\mathcal{O}_{X}(\Gamma) \otimes \mathcal{H}_{W ; \vec{T} ; \gamma}(X) \otimes \mathcal{O}_{X}(-\Gamma)
$$

for $\mathcal{H}_{W ; \vec{T} ; \gamma(\Gamma)}(X)$ viewed as a subalgebra of $k(X)[W]_{\gamma}$. Note that if $\Gamma^{\prime}-\Gamma$ has finite support, then

$$
\begin{aligned}
\mathcal{O}_{X}\left(\Gamma^{\prime}\right) & \otimes \mathcal{H}_{W ; \vec{T} ; \gamma}(X) \otimes \mathcal{O}_{X}\left(-\Gamma^{\prime}\right) \\
& \cong \mathcal{O}_{X}\left(\Gamma^{\prime}-\Gamma\right) \otimes\left(\mathcal{O}_{X}(\Gamma) \otimes \mathcal{H}_{W ; \vec{T} ; \gamma}(X) \otimes \mathcal{O}_{X}(-\Gamma)\right) \otimes \mathcal{O}_{X}\left(\Gamma-\Gamma^{\prime}\right),
\end{aligned}
$$


where the outer twist on the right hand side is the usual twist by a line bundle. We may also define a sheaf $\mathcal{O}_{X}\left(\Gamma^{\prime}\right) \otimes \mathcal{H}_{W ; \vec{T} ; \gamma}(X) \otimes \mathcal{O}_{X}(-\Gamma)$ in this case by $\mathcal{O}_{X}\left(\Gamma^{\prime}-\Gamma\right) \otimes\left(\mathcal{O}_{X}(\Gamma) \otimes \mathcal{H}_{W ; \vec{T} ; \gamma}(X) \otimes\right.$ $\left.\mathcal{O}_{X}(-\Gamma)\right)$.

Proposition 5.21. Let $\vec{T}, \vec{T}^{\prime}$ be two systems of parameters for $W$ on $X$. Then

$$
\mathcal{H}_{W ; \vec{T}+\vec{T}^{\prime} ; \gamma}(X)=\mathcal{O}_{X}\left(-\sum_{\alpha \in \Phi^{+}(W)} T_{\alpha}^{\prime}\right) \otimes \mathcal{H}_{W ; \vec{T}+{ }^{-} \vec{T}^{\prime} ; \gamma}(X) \otimes \mathcal{O}_{X}\left(\sum_{\alpha \in \Phi^{+}(W)} T_{\alpha}^{\prime}\right)
$$

as subalgebras of $k(X)[W]_{\gamma}$.

Proof. This reduces immediately to the corresponding claim in the rank 1 case, where (after twisting by a line bundle to make $\gamma$ trivial) it reads

$$
\mathcal{H}_{A_{1}, T+T^{\prime}}(C)=\mathcal{O}_{C}\left(-T^{\prime}\right) \otimes \mathcal{H}_{A_{1}, T+{ }^{s} T^{\prime}}(C) \otimes \mathcal{O}_{C}\left(T^{\prime}\right) .
$$

For general parameters (such that no two of $T,{ }^{s} T, T^{\prime},{ }^{s} T^{\prime}$ have a common component), this is straightforward: it is easy to see that that $\mathcal{H}_{A_{1}, T+T^{\prime}}(C)$ preserves the subsheaf $\mathcal{O}_{C}\left(-T^{\prime}\right)$, and $\mathcal{H}_{A_{1}, T+^{s} T^{\prime}}(C)$ preserves the subsheaf $\mathcal{O}_{C}\left(-{ }^{s} T^{\prime}\right)$, and this gives both inclusions.

The proof of Proposition 4.34 carries over to give the following.

Proposition 5.22. Suppose that $T_{\alpha}$ and $T_{-\alpha}$ have no common component for any $\alpha \in \Phi(W)$. Then

$$
\mathcal{H}_{W ; \vec{T} ; \gamma}(X)=\mathcal{H}_{W ; \gamma}(X) \cap \mathcal{O}_{X}\left(-\sum_{\alpha \in \Phi^{+}(W)} T_{\alpha}\right) \otimes \mathcal{H}_{W ; \gamma}(X) \otimes \mathcal{O}_{X}\left(\sum_{\alpha \in \Phi^{+}(W)} T_{\alpha}\right) .
$$

We also note the following fact, which allows us to decouple the conditions associated to different parameters.

Proposition 5.23. Suppose that $\vec{T}$ and $\vec{T}^{\prime}$ are such that $T_{\alpha}$ and $T_{\alpha}^{\prime}$ have no common component for any $\alpha$. Then

$$
\mathcal{H}_{W ; \vec{T}+\vec{T}^{\prime} ; \gamma}(X)=\mathcal{H}_{W ; \vec{T} ; \gamma}(X) \cap \mathcal{H}_{W ; \vec{T}^{\prime} ; \gamma}(X) .
$$

Proof. The rank 1 subalgebras on the left are contained in the corresponding subalgebras on the right, so algebra on the left is certainly contained in the intersection on the right. To see equality, we use the Bruhat filtration and observe that each subquotient on the left is the intersection of the corresponding subquotients on the right.

Remark. This easily gives a version of Proposition 5.22 in which $\mathcal{H}_{W ; \vec{T}+\vec{T}^{\prime} ; \gamma}(X)$ is given as an intersection of two twists of $\mathcal{H}_{W ; \vec{T} ; \gamma}(X)$.

The construction of the adjoint in the finite case carries over. Note that the naïve adjoint $\sum_{w} c_{w} w \mapsto \sum_{w} w c_{w}$ induces a natural isomorphism $k(X)[W]_{\gamma}^{\text {op }} \cong k(X)[W]_{\gamma^{-1}}$. (In terms of the sheaf algebra itself, all we are doing is swapping the two factors of $X \times_{S} X$.) To describe how this acts on the Hecke algebras, it will be helpful to denote the formal sum $\sum_{\alpha \in \Phi^{+}(W)}\left(\left[X^{r_{\alpha}}\right]-\vec{T}\right)$ by $D_{w_{0}}(\vec{T})$, and similarly for $D_{w_{0}}$. This of course agrees with the usual notation whenever the longest element $w_{0} \in W$ actually exists.

Proposition 5.24. The naïve adjoint on $k(X)[W]_{\gamma}$ induces an identity

$$
\begin{aligned}
\mathcal{H}_{W ; \vec{T} ; \gamma}(X)^{\mathrm{op}} & =\mathcal{O}_{X}\left(D_{w_{0}}(\vec{T})\right) \otimes \mathcal{H}_{W ; \vec{T} ; \gamma^{-1}}(X) \otimes \mathcal{O}_{X}\left(-D_{w_{0}}(\vec{T})\right) \\
& =\mathcal{O}_{X}\left(D_{w_{0}}\right) \otimes \mathcal{H}_{W ;-} \overrightarrow{T ; \gamma^{-1}}(X) \otimes \mathcal{O}_{X}\left(-D_{w_{0}}\right) .
\end{aligned}
$$

of subalgebras of $k(X)[W]_{\gamma^{-1}}$. 
Proof. Again, this reduces immediately to the rank 1 case.

Diagram automorphisms of course work as well in the infinite case; the only caveat is that unlike in the finite case, a diagram automorphism can fail to preserve the parameters and twisting datum. More generally, if $H$ is a group of automorphisms of $X$ acting as diagram automorphisms of $W$ and preserving the parameters, and there is an $H$-equivariant gerbe $\mathcal{Z}_{h}$ such that ${ }^{h} \gamma_{i} \sim \gamma_{i} \otimes \partial \mathcal{Z}_{h}$ for each $i$, then the corresponding holomorphic crossed product algebra normalizes the Hecke algebra, and we can combine them into a larger algebra associated to the extended Coxeter Group $W \rtimes H$. (In the $C_{n}$ case we consider in detail below, we will see that even the requirement that the parameters be invariant can be finessed.)

Suppose $A$ and $B$ are sheaf algebras, on $X / S$ and $Y / S$ respectively. An $(A, B)$-bimodule is then simply a sheaf bimodule $M$ on $X \times_{S} Y$ equipped with multiplication maps $A \otimes_{X} M \rightarrow M$, $M \otimes_{Y} B \rightarrow M$ making the obvious diagrams commute. (Note that the restriction of $M$ to a compatible pair of localizations is a bimodule over the corresponding restrictions of $A$ and $B$.) The tensor product is then defined in the obvious way, so that we may define induced modules. Restriction is of course also easy to define, though the sheaf form of Frobenius reciprocity is somewhat tricky, as there are difficulties with defining $\mathcal{H o m}$ on sheaf bimodules in general. (The difficulty is that the category of sheaf bimodules is cocomplete, but not complete, and $\mathcal{H}$ om from a direct limit is an inverse limit. Thus the $\mathcal{H o m}$ of sheaf bimodules will still be a quasicoherent sheaf on the relevant fiber product scheme, but may fail to satisfy the finiteness requirement.)

This is not a problem for the analogue of Proposition 4.39; the only change is that $M$ should be replaced by a suitable bimodule. In the finite case, this is no difficulty: when $W$ is finite, any $\mathcal{H}_{W ; \vec{T}}(X)$-module in the usual sense determines a corresponding $\left(\mathcal{H}_{W ; \vec{T}}(X), \mathcal{O}_{X / W}\right)$ bimodule structure.

Proposition 5.25. Suppose $I, J \subset S$ are such that the parabolic subgroups $W_{I}, W_{J}$ are finite. Then for any $\left(\mathcal{H}_{W_{J} ; \vec{T} ; \gamma}(X), \mathcal{O}_{Y}\right)$-bimodule $M$ and any maximal chain in the Bruhat order on ${ }^{I} W^{J}$, the subquotient corresponding to $w \in{ }^{I} W^{J}$ in the resulting filtration of $\operatorname{Res}_{W_{I}}^{W ; \vec{T} ; \gamma} \operatorname{Ind}_{W_{J}}^{W ; \vec{T} ; \gamma} M$ is the $\left(\mathcal{H}_{W_{I} ; \vec{T} ; \gamma}(X), \mathcal{O}_{Y}\right)$-bimodule

$$
\operatorname{Ind}_{W_{I(w)}}^{W_{I} ; \vec{T} ; \gamma} \mathcal{Z}_{\gamma, w}\left(D_{w}(\vec{T})\right) \otimes w \operatorname{Res}_{W_{J(w)}}^{W_{J} ; \vec{T} ; \gamma} M .
$$

We also have a weaker form of Frobenius reciprocity.

Lemma 5.26. For any $Y$, induction and restriction are adjoint functors between the categories of $\left(\mathcal{H}_{W ; \vec{T} ; \gamma}, \mathcal{O}_{Y}\right)$-bimodules and $\left(\mathcal{H}_{W_{I} ; \vec{T} ; \gamma}, \mathcal{O}_{Y}\right)$-bimodules.

Proof. Since both functors are constructed as tensor products, we see that it suffices to construct compatible morphisms

$$
\mathcal{H}_{W ; \vec{T} ; \gamma} \otimes_{\mathcal{H}_{W_{I} ; \vec{T} ; \gamma}} \mathcal{H}_{W ; \vec{T} ; \gamma} \rightarrow \mathcal{H}_{W ; \vec{T} ; \gamma}
$$

and

$$
\mathcal{H}_{W_{I} ; \vec{T} ; \gamma} \rightarrow \operatorname{Res}_{W_{I} ; \vec{T} ; \gamma}^{W} \mathcal{H}_{W ; \vec{T} ; \gamma},
$$

both of which (along with compatibility) follow directly from the fact that $\mathcal{H}_{W_{I} ; \vec{T} ; \gamma}$ is a subalgebra of $\mathcal{H}_{W ; \vec{T} ; \gamma}$.

Corollary 5.27. Let $M$ be a coherent $\left(\mathcal{H}_{W_{I} ; \vec{T} ; \gamma}, \mathcal{O}_{Y}\right)$-bimodule and $N$ an $\left(\mathcal{H}_{W ; \vec{T} ; \gamma}, \mathcal{O}_{Z}\right)$-bimodule. Then the quasicoherent sheaf $\mathcal{H o m}_{\mathcal{H}_{W ; \vec{T} ; \gamma}}\left(\operatorname{Ind}_{W_{I}}^{W ; \vec{T} ; \gamma} M, N\right)$ on $Y \times_{S} Z$ is a sheaf bimodule. 
Proof. Frobenius reciprocity gives (when $Y$ and $Z$ are affine, which we may certainly reduce to)

$$
\mathcal{H} \operatorname{om}_{\mathcal{H}_{W ; \vec{T} ; \gamma}}\left(\operatorname{Ind}_{W_{I}}^{W ; \vec{T} ; \gamma} M, N\right) \cong \mathcal{H}^{\operatorname{lom}_{\mathcal{H} ; \vec{T} ; \gamma}}\left(M, \operatorname{Res}_{W_{I}}^{W ; \vec{T} ; \gamma} N\right)
$$

and the latter is a sheaf bimodule since $M$ is coherent.

Corollary 5.28. Let $M_{I}$ be a coherent $\left(\mathcal{H}_{W_{I} ; \vec{T} ; \gamma}, Y_{I}\right)$-bimodule, $M_{J}$ a coherent $\left(\mathcal{H}_{W_{J} ; \vec{T} ; \gamma}, Y_{J}\right)$ bimodule, and $M$ an $\left(\mathcal{H}_{W ; \vec{T} ; \gamma}, Y\right)$-bimodule. Then there is a natural composition morphism

$$
\begin{aligned}
& \mathcal{H}_{\mathcal{H}_{W ; \vec{T} ; \gamma}}\left(\operatorname{Ind}_{W_{I}}^{W ; \vec{T} ; \gamma} M_{I}, \operatorname{Ind}_{W_{J}}^{W ; \vec{T} ; \gamma} M_{J}\right) \otimes_{Y_{J}} \mathcal{H o m}_{\mathcal{H}_{W ; \vec{T} ; \gamma}}\left(\operatorname{Ind}_{W_{J}}^{W ; \vec{T} ; \gamma} M_{J}, M\right) \\
& \rightarrow \mathcal{H o m}_{\mathcal{H}_{W ; \vec{T} ; \gamma}}\left(\operatorname{Ind}_{W_{I} ; \vec{T} ; \gamma}^{W} M_{I}, M\right)
\end{aligned}
$$

of $\left(Y_{I}, Y\right)$-bimodules, satisfying the obvious associativity relation.

In particular, given an $\left(\mathcal{H}_{W ; \vec{T} ; \gamma}, Y\right)$-bimodule $M$, we may again define an $\left(X / W_{I}, Y\right)$-bimodule $M^{W_{I}}$ as

$$
\mathcal{H o m}_{\mathcal{H}_{W ; \vec{T} ; \gamma}}\left(\operatorname{Ind}_{W_{I}}^{W ; \vec{T} ; \gamma} \mathcal{O}_{X}, M\right) \cong \mathcal{H}_{\mathcal{O}_{\mathcal{H}_{I} ; \vec{T} ; \gamma}}\left(\mathcal{O}_{X}, \operatorname{Res}_{W_{I}}^{W ; \vec{T} ; \gamma} M\right)
$$

This, of course, is essentially just the extension of $\left(\operatorname{Res}_{W_{I}}^{W ; \vec{T} ; \gamma} M\right)^{W_{I}}$ to bimodules in the obvious way.

Now that we have reasonable definitions, most of the calculations we did in the finite case carry over. We find that (assuming $\gamma$ is trivial on $W_{I}$ and $W_{J}$ ) the submodule of $\operatorname{Res}_{W_{I} ; \vec{T} ; \gamma} \operatorname{Ind}_{W J}^{W ; \vec{T} ; \gamma} \mathcal{O}_{X}$ corresponding to any finite Bruhat order ideal has strongly flat invariants for $W_{I}$, and thus

$$
\mathcal{H}_{W, W_{J}, W_{I} ; \vec{T} ; \gamma}(X):=\left(\operatorname{Ind}_{W_{J}}^{W ; \vec{T} ; \gamma} \mathcal{O}_{X}\right)^{W_{I}}
$$

is an $S$-flat sheaf bimodule on $X / W_{I} \times_{S} X / W_{J}$, and this construction commutes with base change. The subquotients in the corresponding Bruhat filtration may all be described in the following way. For each $w \in{ }^{I} W^{J}$, there is a corresponding line bundle $\mathcal{L}_{w}$ on $X / W_{I(w)}$ (constructed from $\vec{T}$ and $\gamma$ ) such that the subquotient is the direct image in $X / W_{I} \times_{S} X / W_{J}$ of the $\left(X / W_{I(w)}, X / W_{J(w)}\right)$-bimodule $\left(1, w^{-1}\right)_{*} \mathcal{L}_{w}$. More precisely, the line bundle $\mathcal{L}_{w}$ is the descent to $X / W_{I(w)}$ of the $\left(W_{I(w)}\right.$-equivariant!) line bundle $\mathcal{Z}_{\gamma, w}\left(D_{w}(\vec{T})\right) \otimes \mathcal{O}_{X}\left(D_{w_{I}}(-\vec{T})-\right.$ $\left.D_{w_{I(w)}}(-\vec{T})\right)$.

If $\Gamma_{I}, \Gamma_{J}$ are $W_{I}, W_{J}$-invariant functions which are even on reflection hypersurfaces and have finitely supported difference, then for any twisting datum $\gamma$ which is trivial on $W_{I}$ and $W_{J}$,

$$
\mathcal{O}_{X}\left(\Gamma_{J}\right) \otimes \mathcal{H}_{W ; \vec{T} ; \gamma} \otimes \mathcal{O}_{X}\left(-\Gamma_{I}\right)
$$

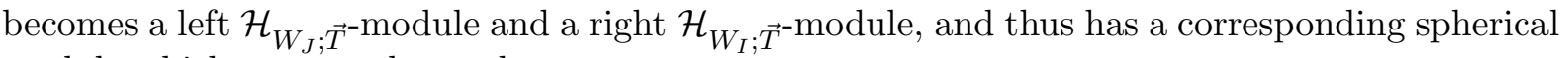
module which we may denote by

$$
\mathcal{O}_{X}\left(\Gamma_{J}\right) \otimes \mathcal{H}_{W, W_{I}, W_{J} ; \vec{T} ; \gamma} \otimes \mathcal{O}_{X}\left(-\Gamma_{I}\right) .
$$

The adjoint takes the following form.

Proposition 5.29. If the root kernels of $W_{I}$ and $W_{J}$ on $X$ are diagonalizable and the twisting datum $\gamma$ is trivial on $W_{I}$ and $W_{J}$, then there is an isomorphism

$$
\mathcal{H}_{W, W_{I}, W_{J} ; \gamma}(X) \cong \mathcal{O}_{X}\left(D_{w_{0}}-D_{w_{I}}\right) \otimes \mathcal{H}_{W, W_{J}, W_{I} ; \gamma^{-1}}(X) \otimes \mathcal{O}_{X}\left(D_{w_{J}}-D_{w_{0}}\right)
$$

which is contravariant with respect to composition. 
Proof. We may view the element $\sum_{w} w$ as a section of $\mathcal{H}_{W_{I}}(X) \otimes \mathcal{O}_{X}\left(-D_{w_{I}}\right)$, since it takes sections of $\mathcal{O}_{X}\left(D_{w_{I}}\right)$ to sections of $\mathcal{O}_{X}$. We thus have an embedding

$$
\mathcal{H}_{W, W_{I} ; \gamma}(X) \rightarrow \mathcal{H}_{W ; \gamma}(X) \otimes \mathcal{O}_{X}\left(-D_{w_{I}}\right)
$$

acting as

$$
\sum_{w \in W^{I}} c_{w W_{I}} w w_{I} \mapsto \sum_{w \in W} c_{w W_{I}} w
$$

Moreover, if the original operator is a local section of $\mathcal{H}_{W, W_{I}, W_{J} ; \gamma}(X)$, then we have

$$
c_{w^{\prime} w W_{I}}={ }^{w^{\prime}} c_{w W_{I}}
$$

for $w^{\prime} \in W_{J}$, so that we may write the image as

$$
\sum_{w^{\prime} \in W_{J}} w^{\prime} \sum_{w \in{ }^{J} W} c_{W_{J} w W_{I}} w
$$

Taking the adjoint (including the twist by $\mathcal{O}_{X}\left(D_{w_{0}}\right)$ ) gives

$$
\sum_{w^{\prime} \in W_{J}} \sum_{w \in J^{J} W}(-1)^{\ell\left(w w^{\prime}\right)} w^{-1} c_{W_{J} w W_{I}} w^{\prime-1}=\sum_{w \in W^{J}} \sum_{w^{\prime} \in W_{J}}(-1)^{\ell\left(w w^{\prime}\right)} w c_{W_{J} w^{-1} W_{I}} w^{\prime}
$$

in $\mathcal{O}_{X}\left(-D_{w_{I}}\right) \otimes \mathcal{H}_{W ; \gamma^{-1}}(X)$. Right dividing by $\sum_{w^{\prime} \in W_{J}}(-1)^{\ell\left(w^{\prime}\right)} w^{\prime}$ gives a section of

$$
\mathcal{O}_{X}\left(-D_{w_{I}}\right) \otimes \mathcal{H}_{W, W_{J}, W_{I} ; \gamma^{-1}}(X) \otimes \mathcal{O}_{X}\left(D_{w_{J}}\right)
$$

as required. Compatibility with composition follows by observing that in a composition, the factor $\sum_{w \in W_{I}} w$ needed in the middle is already present in the other operator, and the factor $\sum_{w \in W_{J}}(-1)^{\ell(w)} w$ that should be removed is needed in the other operator.

Proposition 5.30. If the root kernels of $W_{I}$ and $W_{J}$ on $X$ are diagonalizable, then

$$
\begin{aligned}
\mathcal{H}_{W, W_{I}, W_{J} ; \vec{T} ; \gamma}(X) & \subset \mathcal{H}_{W, W_{I}, W_{J} ; \gamma}(X) \\
& \cap \mathcal{O}_{X}\left(\sum_{\alpha \in \Phi^{-}(W) \backslash \Phi^{-}\left(W_{J}\right)} T_{\alpha}\right) \otimes \mathcal{H}_{W, W_{I}, W_{J} ; \gamma}(X) \otimes \mathcal{O}_{X}\left(-\sum_{\alpha \in \Phi^{-}(W) \backslash \Phi^{-}\left(W_{I}\right)} T_{\alpha}\right),
\end{aligned}
$$

with equality unless there is a root $\alpha$ such that $T_{\alpha}$ and $T_{-\alpha}$ have a common component.

Proof. Over the locus of $S$ covered by symmetric idempotents, we may use those idempotents to locally identify $\mathcal{H}_{W, W_{I}, W_{J} ; \vec{T} ; \gamma}(X)$ with a submodule of $\mathcal{H}_{W, W_{I}, W_{J} ; \gamma}(X)$ (using the same idempotent to embed both in $\left.\mathcal{H}_{W ; \gamma}(X)\right)$. This identification is compatible with the identification of meromorphic fibers, so extends to a global identification on each fiber covered by symmetric idempotents, and from there to the closure of the symmetric idempotent locus.

Similarly, local idempotents embed $\mathcal{H}_{W, W_{I}, W_{J} ; \vec{T} ; \gamma}(X)$ in

$$
\mathcal{O}_{X}\left(\sum_{\alpha \in \Phi^{-}(W)} T_{\alpha}\right) \otimes \mathcal{H}_{W ; \gamma}(X) \otimes \mathcal{O}_{X}\left(-\sum_{\alpha \in \Phi^{-}(W)} T_{\alpha}\right),
$$

and the idempotents eliminate the contributions of $T_{\alpha}$ for $\alpha \in W_{I}, W_{J}$ respectively.

To see that the inclusion is tight, we need merely verify that both sides have the same Bruhat subquotients, which reduces to verifying that the negative part of

$$
\sum_{\alpha \in \Phi^{+}(W) \cap w \Phi^{-}(W)}\left(T_{-\alpha}-T_{\alpha}\right)+\sum_{\alpha \in \Phi^{-}\left(W_{J}\right) \backslash \Phi^{-}\left(W_{J} \cap w W_{I} w^{-1}\right)}\left(T_{w \alpha}-T_{\alpha}\right)
$$

has no further cancellation, just as in the finite case. 
Corollary 5.31. Let $\vec{T}$ be a system of parameters such that every $T_{\alpha}$ is transverse to every reflection hypersurface. If the root kernels of $W_{I}$ and $W_{J}$ are diagonalizable, then $\mathcal{H}_{W, W_{I}, W_{J} ; \vec{T} ; \gamma}(X)$ may be identified with the sheaf sub-bimodule of $\mathcal{H}_{W, W_{I}, W_{J} ; \gamma}(X)$ consisting of operators $\sum_{w} c_{w} w W_{I}$ such that for every $w, c_{w}$ vanishes on the divisor

$$
\sum_{\alpha \in \Phi^{+}(W) \cap w \Phi^{-}(W)} T_{\alpha}+\sum_{\alpha \in \Phi^{-}\left(W_{J}\right) \backslash \Phi^{-}\left(W_{J} \cap w W_{I} w^{-1}\right)} T_{\alpha} .
$$

Corollary 5.32. If the root kernels of $W_{I}$ and $W_{J}$ on $X$ are diagonalizable, then there is an isomorphism

$$
\begin{aligned}
\mathcal{H}_{W, W_{I}, W_{J} ; \vec{T} ; \gamma}(X) \cong & \mathcal{O}_{X}\left(D_{w_{0}}(-\vec{T})-D_{w_{I}}(-\vec{T})\right) \otimes \mathcal{H}_{W, W_{J}, W_{I} ; \vec{T} ; \gamma^{-1}}(X) \\
& \otimes \mathcal{O}_{X}\left(D_{w_{J}}\left({ }^{-} \vec{T}\right)-D_{w_{0}}(-\vec{T})\right)
\end{aligned}
$$

which is contravariant with respect to composition.

Remark. Of course, we also have analogous results for the other three natural Hom sheaves discussed above.

We close by considering the analogue in this setting of the residue conditions of [11]. It suffices to consider the algebras $\mathcal{H}_{W, W_{I}, W_{J} ; \gamma}(X)$, since $\vec{T}$ simply imposes generic vanishing conditions on the coefficients, as already discussed. (And, of course, this includes the Hecke algebras themselves by taking $I=J=\varnothing$.) We may further assume $J=\varnothing$, as $\mathcal{H}_{W, W_{I}, W_{J} ; \gamma}(X)$ is the submodule of $\mathcal{H}_{W, W_{I} ; \gamma}(X)$ consisting of $W_{J}$-invariant operators.

Since we may embed $\mathcal{H}_{W, W_{I} ; \gamma}(X)$ in $\mathcal{H}_{W ; \gamma}(X)$ using a symmetric idempotent, the fact that the latter is spanned by submodules

$$
\mathcal{H}_{\langle r\rangle ; \gamma}(X) \mathcal{O}_{X}[W]_{\gamma}
$$

implies something similar for the former: it is spanned by the submodules

$$
\mathcal{H}_{\langle r\rangle ; \gamma}(X) \bigoplus_{w \in W^{I}} \mathcal{Z}_{w} w W_{I}
$$

If $r w W_{I}=w W_{I}$, then we may rewrite the corresponding summand as

$$
\mathcal{Z}_{w} w \mathcal{H}_{\left\langle w^{-1} r w\right\rangle ; \gamma}(X) W_{I}=\mathcal{Z}_{w} w W_{I},
$$

and thus we may omit any such summand.

If we restrict to a finite subset $S \subset W / W_{I}$, then we conclude that

$$
c_{w W_{I}} \in \mathcal{Z}_{w}\left(\sum_{r \in R(W): r w W_{I} \in S \backslash\left\{w W_{I}\right\}}\left[X^{r}\right]\right)
$$

and that there is a residue condition relating $c_{w W_{I}}$ and $c_{r w W_{I}}$ along $\left[X^{r}\right]$. Again moving $\mathcal{Z}_{w} w$ to the left lets us express this condition in the form

$$
c_{w}+{ }^{w} h_{w^{-1} r w} \zeta_{w, w^{-1} r w}^{-1} c_{r w}=\left.0 \in \mathcal{Z}_{w}\left(\left[X^{r}\right]\right)\right|_{\left[X^{r}\right]},
$$

where $h_{w^{-1} r w}$ comes from the root datum on $\left\langle w^{-1} r w\right\rangle$. Note that we could also determine this by right dividing by $f_{w} w W_{I}$, where $f_{w}$ trivializes $\mathcal{Z}_{w}$ in a neighborhood of $X^{r}$ to obtain the condition in the form

$$
c_{w}+f_{w}{ }^{r} f_{w}^{-1} h_{r} \zeta_{r, w}^{-1} c_{r w}=\left.0 \in \mathcal{Z}_{w}\left(\left[X^{r}\right]\right)\right|_{\left[X^{r}\right]} .
$$

Of course, these are equivalent (since otherwise $\gamma$ would violate the compatibility conditions).

Naturally, in the untwisted case, the condition is just that $c_{w}+c_{r w}$ is holomorphic (essentially Corollary 4.3), and the same holds (along $\left[X^{r}\right]$ ) if we embed $\mathcal{H}_{W, W_{I} ; \gamma}(X)$ in $k(X)\left[W / W_{I}\right]$ via an expression of the twisting datum as the coboundary of a formal divisor which is transverse to the reflection hypersurfaces. 


\section{The (double) affine case}

The most interesting case for our purposes is when the Coxeter group is an affine Weyl group $\widetilde{W}$. We actually want to modify the construction (very) slightly in that case, as the abelian variety being acted on is slightly larger than we would like. That is, rather than have an $(n+1)$ dimensional variety with an invariant map to an elliptic curve, we would prefer to act on the fibers of that map. If we pull back the sheaf bimodule $\mathcal{H}_{\widetilde{W} ; \vec{T} ; \gamma}(X)$ from $X \times_{S} X$ to $X \times \times_{X / A_{\widetilde{W}}} X$, then we find (by considering what happens on invariant localizations, say) that the result is still naturally a sheaf algebra. The group no longer acts faithfully on every fiber, but the various calculations involving the Bruhat filtration carry over without difficulty, so that we still obtain a flat family of sheaf algebras generated by the rank 1 subalgebras. One caveat is that $T_{\alpha}$ and $T_{\beta}$ need not be transverse for $\alpha \neq \pm \beta$; if they correspond to the same root of the finite root system, then their divisors differ only by a translation, which may act trivially on some fibers.

Still, we have the following definition. First, if $X$ is a torsor over the abelian scheme $A$, an action of $\widetilde{W}$ on $X$ by affine reflections is simply an action such that every simple reflection fixes a hypersurface and the action on $A$ factors through a faithful action of the corresponding finite Weyl group. Any such action arises from an action of coroot type by specializing the parameter $q$. (Recall that $q=\zeta(z)$ gives the image of the origin of a fiber under the special reflection $s_{0}$.) In addition, every finite parabolic subgroup still acts faithfully (regardless of $q$ ), and thus in particular we still have good notions of systems of parameters and twisting data. The one caution is that when expressing twisting data as a coboundary of some formal sum of divisors, one needs to assume $q$ non-torsion. This is not truly an issue, however, as one can simply take the limit of the twisting data from the non-torsion case (which simply adds an extra level of formality to the formal sum). In particular, the cocycles in Cartier divisors associated to the formal sums $\sum_{\alpha \in \Phi^{+}(W)} T_{\alpha}$ and $\sum_{\alpha \in \Phi^{+}(W)}\left[X^{r_{\alpha}}\right]$ remain cocycles after specializing $q$.

Definition 6.1. Let $\widetilde{W}$ act on $X$ by affine reflections, let $\vec{T}$ be a system of parameters, and let $\gamma$ be a twisting datum. The corresponding elliptic double affine Hecke algebra $\mathcal{H}_{\widetilde{W} ; \vec{T} ; \gamma}(X)$ is the sheaf subalgebra of $k(X)[\widetilde{W}]_{\gamma}$ generated by the rank 1 subalgebras $\mathcal{H}_{\langle s\rangle ; \vec{T} ; \gamma}(X)$ for $s \in S$.

Proposition 6.2. The subsheaf of $\mathcal{H}_{\widetilde{W} ; \vec{T} ; \gamma}(X)$ corresponding to any finite Bruhat order ideal is an $S$-flat coherent sheaf on $X \times_{S} X$.

Proposition 6.3. We have

$$
\mathcal{H}_{\widetilde{W} ; \vec{T} ; \gamma}(X) \subset \mathcal{H}_{\widetilde{W} ; \gamma}(X) \cap \mathcal{O}_{X}\left(-\sum_{\alpha \in \Phi^{+}(\widetilde{W})} T_{\alpha}\right) \otimes \mathcal{H}_{\widetilde{W} ; \gamma}(X) \otimes \mathcal{O}_{X}\left(\sum_{\alpha \in \Phi^{+}(\widetilde{W})} T_{\alpha}\right)
$$

with equality unless there are $\alpha \in \Phi^{+}(\widetilde{W}), \beta \in \Phi^{-}(\widetilde{W})$ such that $T_{\alpha}$ and $T_{\beta}$ have a common component.

Remark. When $q$ is torsion, then in fact each $\alpha$ has infinitely many $\beta$ (both positive and negative) such that $T_{\alpha}=T_{\beta}$, and thus the hypothesis for equality is never satisfied.

Corollary 6.4. Let $\vec{T}$ be a system of parameters such that every $T_{\alpha}$ is transverse to every reflection hypersurface. Then $\mathcal{H}_{\widetilde{W} ; \vec{T} ; \gamma}(X)$ may be identified with the sheaf subalgebra of $\mathcal{H}_{\widetilde{W} ; \gamma}(X)$ consisting locally of operators $\sum_{w} c_{w} w$ such that for every $w, c_{w}$ vanishes on the divisor $\sum_{\alpha \in \Phi^{+}(W) \cap w \Phi^{-}(W)} T_{\alpha}$.

It will be helpful to understand the possible twisting data in this scenario. If we assume that $W$ has trivial root kernel, then we may trivialize the twisting datum along $W$, and it thus 
remains to determine the possibilities for the restriction to $s_{0}$. If $\widetilde{W} \neq \tilde{A}_{n}$, then the affine diagram is a tree with $s_{0}$ as a leaf, and thus $s_{0}$ commutes with a rank $n-1$ parabolic subgroup of $W$. The polarization of $\mathcal{Z}_{s_{0}}$ must be invariant under that subgroup, and since it is also negated by $s_{0}$, we find that $\mathcal{Z}_{s_{0}}$ must have trivial polarization. To fully specify the twisting datum, we need to choose a solution of $\mathcal{L} \otimes{ }^{s_{0}} \mathcal{L}^{-1}=\mathcal{Z}_{s_{0}}$. If $\widetilde{W} \neq \tilde{C}_{n}$, so that $s_{0}$ is connected to the finite diagram via an ordinary edge, then we find that the polarization of $\mathcal{L}$ is the sum of a polarization on the connected kernel of the coroot map and a polarization in the image of $1+s_{0}$; it thus follows that we may replace $\mathcal{L}$ itself by a line bundle with trivial polarization without affecting the root datum.

In other words, for $\widetilde{W}$ not of type $A$ or $C$, any twisting datum is (up to an overall twist by a line bundle) given by taking the twisting datum along $s_{0}$ to be the coboundary of a point in $\operatorname{Pic}^{0}(X)$. For type $C$, there is at most one additional component which may be reached if it exists by taking the coboundary of the pullback of a degree 1 line bundle on the coroot curve. For type $A$, the situation is more complicated, as it turns out that there are in fact twisting data with nontrivial polarizations. Luckily, these can always be described via cocycles in Cartier divisors. Type $A_{n}$ corresponds to the action of $S_{n+1}$ on the sum zero subvariety of $E^{n+1}$, with $s_{0}$ acting as $\left(z_{1}, z_{n+1}\right) \mapsto\left(q+z_{n+1}, z_{1}-q\right)$. For any point $u \in E$, we may consider the formal sum

$$
\sum_{j \geq 0} \sum_{1 \leq i \leq n+1}\left[z_{i}=u+j q\right]
$$

This is invariant under the finite Weyl group, while its coboundary under $s_{0}$ is $\left[z_{1}=u\right]-\left[z_{n+1}=\right.$ $u+q]$. This gives a well-defined twisting datum for generic $u, q$, and thus extends to all $u, q$. As $u$ varies, these cover the corresponding component of the group scheme classifying twisting data trivial on $W$, and one readily verifies that this component generates the full group scheme.

In the $\tilde{A}_{n}$ case, we could obtain every twisting datum via a cocycle in Cartier divisors. This is unlikely to hold in general type (albeit without a known counterexample), but something only slightly weaker is true.

Proposition 6.5. Let $X / S$ be an abelian torsor equipped with an action of $\widetilde{W}$ of coroot type such that $W$ has trivial root kernel. Then any twisting datum on $X / S$ can be fppf locally represented by a cocycle in Cartier divisors.

Proof. Certainly the twist by a line bundle may be represented as a cocycle in Cartier divisors, so we reduce to the case that the twisting datum is trivial along $W$. Let $\lambda_{0}: X \rightarrow E^{\prime}$ be a (nonconstant) homomorphism from $X$ to an elliptic curve, and consider the orbit $\widetilde{W} \lambda_{0}$ of such maps for general $u \in E^{\prime}$. There is a point $q^{\prime} \in E^{\prime}$ (determined from $q$ and $\lambda_{0}$ ) such that $\lambda$ is in the orbit iff

$$
\lambda=w \lambda_{0}+j q^{\prime}
$$

for some $w \in W, j \in \mathbb{Z}$. Now, consider the formal sum

$$
\sum_{j \geq 0} \sum_{\lambda \in W \lambda_{0}}\left[w \lambda_{0}=u+j q^{\prime}\right]
$$

of divisors on $X$. This is $W$-invariant, and its coboundary with respect to $s_{0}$ has finite support, so we obtain a well-defined family of cocycles in Cartier divisors.

Apart from type $A$ and $C$, it will suffice to show that it depends nontrivially on $u$ (since then we have a nonconstant morphism from $E^{\prime}$ to the connected 1-parameter group scheme parametrizing twisting data). Such dependence is clearly independent of $q$, so we may assume $q$ non-torsion, and thus $q^{\prime}$ non-torsion. Then shifting $u$ by $q^{\prime}$ above subtracts $\sum_{\lambda \in W \lambda_{0}}\left[w \lambda_{0}=u\right]$ 
from the formal sum. This divisor class is $W$-invariant and ample, so is not $s_{0}$-invariant, and thus shifting $u$ has a nontrivial effect on the twisting datum as required.

In type $C$, the same argument shows that everything in the identity component of the scheme parametrizing twisting data is fppf locally represented by cocycles in Cartier divisors, and one can explicitly verify (indeed, see the discussion of the $\tilde{C}$ case below) that when there is another component, it can still be reached in this way.

Remark 6.6. The presence of exotic twisting data in type $A$ can be explained by considering the induced cocycle in polarizations. As discussed in more detail in the $\tilde{C}_{n}$ case below, such a cocycle for arbitrary type may be obtained as the coboundary of a $W$-invariant rational homogeneous polynomial of the form $p_{3}(\vec{z}, q) / q$ ) (with appropriate modifications in the presence of nontrivial isogenies). The only part that contributes to the polarization of the coboundary is the part of degree 3 in $\vec{z}$, and the only indecomposable finite Weyl groups with invariants of degree 3 are those of type $A_{n}$ for $n \geq 2$.

Remark 6.7. In the above argument, we used the fact that shifting $u$ by $q^{\prime}$ had the effect of twisting by a $W$-invariant line bundle, and that this had a nontrivial effect on the twisting datum. It follows that we do not have a well-defined scheme parametrizing twisting data modulo twists by line bundles. Indeed, it follows that for $q$ non-torsion, twists by line bundles are dense in the identity component of the group scheme of twisting data trivial on $W$.

It is unclear (but likely) if this result holds for actions with nontrivial root kernel. This fact is useful enough, however, that we will include it as an implicit assumption below; that is, we will impose as an additional condition that the twisting datum is fppf locally represented by a cocycle in Cartier divisors, or equivalently (by the above argument) that the restriction of the twisting datum to $W$ is a coboundary.

The description of $\mathcal{H}_{\widetilde{W}}(X)$ (or $\mathcal{H}_{\widetilde{W}}, \widetilde{W}_{I}, \widetilde{W}_{J}(X)$ ) as holomorphy-preserving operators continues to hold, as long as $\widetilde{W}$ acts faithfully, or more generally for any Bruhat order ideal that injects in $\operatorname{Aut}(X)$. So we may again apply Corollary 4.8 to obtain residue conditions analogous to those of [11] for the finite case, just as in the non-affine case.

For the twisted case, we note that when $q$ is nontorsion, the algebra $\mathcal{H}_{\widetilde{W} ; \gamma}(X)$ is still an algebra of the type constructed in Proposition 5.13, so there is no difficulty in generalizing the proofs and we still have reasonable residue conditions. (If we represent the twisting datum as a cocycle in Cartier divisors transverse to the reflection hypersurfaces, then the corresponding embedding in $k(X)[\widetilde{W}]$ is again holomorphy-preserving away from the support of the cocycle, and thus the residue conditions can still be obtained from Corollary 4.8. Of course, the argument we used in the non-affine case works equally well!) Any Bruhat interval is flat as $q$ varies, and thus we can obtain conditions for torsion $q$ by taking limits. This can become quite complicated when the Bruhat interval is large compared to the order of $q$, but in the case that the Bruhat interval acts faithfully, there is no difficulty taking the limit; the poles are at most simple, and one simply has the usual condition along each reflection hypersurface given by the twisting datum. The same applies to spherical modules; again, the sheaf on each Bruhat interval can be obtained as the limit from general $q$, and the limit is straightforward as long as there is no coefficient such that two components of its allowed polar divisor coalesce in the limit. In other words, for the $W_{I^{-}}$-invariant Bruhat interval $\left[\leq w W_{I}\right]$, if the set of reflections $\left\{r \in R(W): r w^{\prime} W_{I} \in\left[\leq w W_{I}\right] \backslash\left\{w^{\prime} W_{I}\right\}\right\}$ injects in $\operatorname{Aut}(X)$ for every $w^{\prime} W_{I} \leq w W_{I}$, then the residue conditions are precisely as expected from the non-affine case.

The most important case for the spherical algebra construction is when $\widetilde{W}_{I}=W$ is the corresponding finite Weyl group. In that case, we note that each coset $\widetilde{W} / W$ contains a unique translation, and thus we may interpret elements of the spherical algebra as (elliptic) difference operators, with $\mathcal{H}_{\widetilde{W}, W}(X)$ for non-torsion $q$ corresponding to difference operators that (locally) 
preserve $W$-invariant holomorphic functions. Note that the Bruhat order on $W \backslash \widetilde{W} / W$ is simply the usual dominance order on dominant weights [19].

This has the following consequence. We say that a sheaf algebra is a domain if the product of a nonzero section on $U \times V$ and a nonzero section on $V \times W$ is always a nonzero section on $U \times W$.

Proposition 6.8. Suppose that the root kernel for $W$ on $X$ is diagonalizable. Then for any twisting datum $\gamma$ which is trivial on $W$, every fiber of the spherical algebra $\mathcal{H}_{\widetilde{W}, W ; \vec{T} ; \gamma}(X)$ is a domain.

Proof. We first note that the inclusion of $\mathcal{H}_{\widetilde{W}, W ; \vec{T} ; \gamma}(X)$ in $k(X)[\Lambda]_{\gamma}$ is injective on fibers. This follows from the fact that we can compute $\mathcal{H}_{\widetilde{W}, W ; \vec{T} ; \gamma}(X)$ as the $W$-invariant submodule of an $S$ flat module with strongly flat invariants, and thus the inclusion $\mathcal{H}_{\widetilde{W}, W ; \vec{T} ; \gamma}(X) \subset \operatorname{Ind}_{W} \widetilde{W} ; \vec{T} ; \gamma \mathcal{O}_{X}$ is injective on fibers; as the induced module injects in the induced module of $k(X)$ and this equals $k(X)[\Lambda]_{\gamma}$, the desired injectivity follows.

In particular, any local section of a fiber on a product of $W$-invariant open subsets can be identified with a $W$-invariant section of $k(X)[\Lambda]_{\gamma}$. Since this identification is compatible with the multiplication, it will suffice to show that $k(X)[\Lambda]_{\gamma}$ is a domain. Since $\Lambda$ is a finitely generated free abelian group, there exist injective homomorphisms $\Lambda \rightarrow \mathbb{R}$, allowing us to define a total ordering on $\Lambda$ compatible with the group law. In particular, for any nonzero element $\sum_{\lambda \in \Lambda} c_{\lambda}[\lambda]$ of $k(X)[\Lambda]_{\gamma}$, there is a corresponding notion of "leading monomial", defined as $c_{\lambda}[\lambda]$, where $\lambda$ is the largest element of the support. If $f$ has leading monomial $f_{\lambda}[\lambda]$ and $g$ has leading monomial $g_{\mu}[\mu]$, then $f g$ has leading monomial $\zeta_{\lambda \mu}\left(f_{\lambda} \otimes{ }^{\lambda} g_{\mu}\right)$, and is therefore nonzero as required.

Another important feature of the spherical algebra in the affine case is that it is Morita equivalent to the DAHA itself, at least for generic parameters. The proof relies on the following result on two-sided ideals in the DAHA.

Lemma 6.9. Let $k$ be an algebraically closed field, and suppose $\widetilde{W}$ acts faithfully on the abelian torsor $Y / k$. Let $S$ be a product of symmetric powers of coroot curves of $Y$, and let $\vec{T}$ be the corresponding universal system of parameters on $X:=Y \times S$. Then for any ideal sheaf $\mathcal{I} \subset \mathcal{O}_{X}$, there is a dense open subset of $S$ on which $\mathcal{I}$ generates $\mathcal{H}_{\widetilde{W} ; \vec{T} ; \gamma}(X)$ as a two-sided ideal.

Proof. Suppose otherwise. We may assume that

$$
\mathcal{I}=\left.\left(\mathcal{H}_{\widetilde{W} ; \vec{T} ; \gamma}(X) \mathcal{I H}_{\widetilde{W} ; \vec{T} ; \gamma}(X)\right)\right|_{1},
$$

since both sides generate the same two-sided ideal. If we replace each instance of the DAHA by the restriction of the DAHA to operators supported entirely on $s_{i}$, it follows that

$$
\mathcal{I} \supset{ }^{s_{i}} \mathcal{I} \otimes \mathcal{O}_{X}\left(T_{i}+{ }^{s_{i}} T_{i}\right)
$$

This in turn induces a weak symmetry condition on the set $Z$ cut out by $\mathcal{I}$ : if $x \in Z$, then either $s_{i}(x) \in Z$ or $x \in T_{i} \cup{ }^{s_{i}} T_{I}$, and in those terms our goal is to prove that $Z$ does not meet the generic fiber.

We claim that this is true for any proper closed subset of $X$ satisfying this condition. Since the weak symmetry condition is preserved under restriction to the generic fiber of $S$ as well as under taking Zariski closure, we may assume that every component of $Z$ meets the generic fiber, and thus by properness that it meets every fiber. Now consider a fiber on which each $T_{i}$ is contained (as a set) in $\left[Y^{s_{i}}\right]$. If $x$ is a point of $Z$ in such a fiber, the weak symmetry condition implies that either $s_{i}(x) \in Z$ or $x \in\left[Y^{s_{i}}\right]$ and thus $x=s_{i}(x)$. Thus the restriction of $Z$ to such 
a fiber must be $s_{i}$-invariant (as a set!) and thus $\widetilde{W}$-invariant. Since $\widetilde{W}$ acts faithfully on $Y$, any $\widetilde{W}$-orbit in $Y$ is Zariski dense, and thus $Z$ must contain every such fiber.

It will thus suffice to prove that $Z$ cannot contain any fiber, and thus obtain a contradiction. Again, since $\widetilde{W}$ acts faithfully, $Z_{k(S)}$ cannot contain any $\widetilde{W}$-orbits, and thus for any point $x \in Z_{k(S)}$, there exists $w \in \widetilde{W}$ and $i \in\{0, \ldots, n\}$ such that $w x \in Z_{k(S)}$ but $s_{i} w x \notin Z_{k(S)}$. It follows that $w x \in T_{i} \cup{ }^{s_{i}} T_{i}$, and thus $Z_{k(S)}$ is covered by the sets of the form ${ }^{w} T_{i}$ for $w \in \widetilde{W}$, $i \in\{0, \ldots, n\}$. Since every component of $Z$ meets the generic fiber, $Z$ is covered by the same sets; since none of these sets contains a fiber, $Z$ cannot contain a fiber, and we are done.

Proposition 6.10. Suppose $\widetilde{W}$ acts faithfully on $X$ such that the root kernel of $W$ on $X$ is diagonalizable and $\gamma$ is trivial on $W$. If $\vec{T}$ is in sufficiently general position, then the categories of $\mathcal{H}_{\widetilde{W} ; \vec{T} ; \gamma}(X)$-modules and $\mathcal{H}_{\widetilde{W}, W ; \vec{T} ; \gamma}(X)$-modules are equivalent, with the inverse equivalences given by $-W$ and $\operatorname{Ind}_{W}^{\widetilde{W} ; \vec{T} ; \gamma} \mathcal{O}_{X} \otimes_{\mathcal{H}_{\widetilde{W}, W ; \vec{T} ; \gamma}(X)}-$.

Proof. Since we are assuming $\vec{T}$ is in sufficiently general position, we may in particular assume that the finite Hecke algebra $\mathcal{H}_{W ; \vec{T}}(X)$ has a covering by symmetric idempotents, so that both functors are exact. It suffices to check that they are inverses on the regular representation. One direction is by definition of the spherical algebra, so we reduce to showing that the natural map

$$
\phi: \operatorname{Ind}_{W}^{\widetilde{W} ; \vec{T} ; \gamma} \mathcal{O}_{X} \otimes_{\mathcal{H}_{\widetilde{W}, W ; \vec{T} ; \gamma}(X)} \mathcal{H}_{\widetilde{W} ; \vec{T} ; \gamma}(X)^{W} \rightarrow \mathcal{H}_{\widetilde{W} ; \vec{T} ; \gamma}(X)
$$

is surjective. The image certainly contains the image of

$$
\mathcal{O}_{X} \otimes_{\mathcal{H}_{W, W ; \vec{T}}(X)} \mathcal{H}_{W ; \vec{T}}(X)^{W} \rightarrow \mathcal{H}_{W ; \vec{T}}(X) .
$$

This map is almost certainly not surjective, but its cokernel is torsion, since the analogous map for $k(X)[W]_{\gamma}$ is surjective. It follows that the image meets $\mathcal{O}_{X}$ nontrivially, and thus the same is true for $\phi$. But then the image of $\phi$ is a two-sided ideal in the DAHA meeting $\mathcal{O}_{X}$ nontrivially, and thus the lemma tells us that $\phi$ is surjective.

Remark. More generally, if $\vec{T}$ and $\vec{T}^{\prime}$ are two systems of parameters in sufficiently general position, the same argument tells us that $\mathcal{H}_{\tilde{W} ;-\vec{T}+\vec{T}^{\prime} ; \gamma}(X)$ is Morita equivalent to

$$
\operatorname{End}\left(\operatorname{Ind}_{W}^{\widetilde{W} ; \vec{T}+\vec{T}^{\prime} ; \gamma} \mathcal{O}_{X}\left(-\sum_{\alpha \in \Phi^{+}(W)} T_{\alpha}\right)\right) \text {. }
$$

(Note that by Proposition 5.21, this is indeed a module, and is cut out by the image of local symmetric idempotents in $\mathcal{H}_{W ;-\vec{T}+\vec{T}^{\prime}}(X)$.)

In the affine case, the spherical algebra has an additional symmetry. As we mentioned above, for general Coxeter groups, the usual symmetry replacing $\vec{T}$ by ${ }^{-} \vec{T}$ has an issue in the spherical algebra case. The proof of that symmetry relied on the fact that $\sum_{\alpha \in \Phi(W)} T_{\alpha}$ has no effect on twisting, so that twisting by $\sum_{\alpha \in \Phi^{+}(W)} T_{\alpha}$ and $-\sum_{\alpha \in \Phi^{-}(W)} T_{\alpha}$ have the same effect, letting one move the twist to the other half of the intersection. For the spherical algebra, this operation instead turns $-\sum_{\alpha \in \Phi^{-}(W) \backslash \Phi^{-}\left(W_{I}\right)} T_{\alpha}$ into $\sum_{\alpha \in \Phi^{+}(W) \cup \Phi^{-}\left(W_{I}\right)} T_{\alpha}$, and thus does not give an algebra of the same form (but rather involves the other natural representation $\left.\mathcal{O}_{X}\left(-\sum_{\alpha \in \Phi^{+}\left(W_{I}\right)} T_{\alpha}\right)\right)$. However, in the affine case, it turns out that there actually is a system of parameters $\vec{T}^{\prime}$ such that

$$
\sum_{\alpha \in \Phi^{+}(\widetilde{W}) \cup \Phi^{-}(W)} T_{\alpha}=\sum_{\alpha \in \Phi^{-}(\widetilde{W}) \backslash \Phi^{-}(W)} T_{\alpha}^{\prime} .
$$


In both cases, we can break up the sum as a sum over roots of the finite Weyl group $W$, and find that on the left-hand side we have a sum over translates of $T_{\alpha}$ by nonnegative multiples of some $q_{\alpha}$, while on the right-hand side we have a sum over translates of $T_{\alpha}^{\prime}$ by negative multiples of $q_{\alpha}$. We may thus simply take $T_{\alpha}^{\prime}$ to be the translate of $T_{-\alpha}$ by $q_{\alpha}$.

As a result, we find that the algebra

$$
\mathcal{H}_{\operatorname{om}_{\mathcal{H}} ; \vec{T}(X)}\left(\operatorname{Ind}_{W}^{\widetilde{W} ; \vec{T} ; \gamma} \mathcal{O}_{X}\left(-\sum_{\alpha \in \Phi^{+}(W)} T_{\alpha}\right), \operatorname{Ind}_{W}^{\widetilde{W} ; \vec{T} ; \gamma} \mathcal{O}_{X}\left(-\sum_{\alpha \in \Phi^{+}(W)} T_{\alpha}\right)\right)
$$

may be identified with an instance of $\mathcal{H}_{\widetilde{W}, W ; \vec{T}}(X)$ in which the parameters have been translated by $q_{\alpha}$. We also obtain a pair of (strongly flat) intertwining bimodules as discussed above in the finite case. For generic parameters, each of the algebras is Morita equivalent to the original DAHA by Proposition 6.10 and the remark following, and thus the algebras are Morita equivalent to each other, with the equivalences induced by the corresponding bimodules. More generally, if we write $\vec{T}=\vec{T}^{\prime}+\vec{T}^{\prime \prime}$, then the algebra obtained by translating $\vec{T}^{\prime \prime}$ but leaving $\vec{T}^{\prime}$ alone is still generically expressible as the endomorphism ring of an induced representation (coming from $\mathcal{O}_{X}\left(-\sum_{\alpha \in \Phi^{+}(W)} \vec{T}_{\alpha}^{\prime}\right)$ ), so the proof of Proposition 6.10 still gives a Morita equivalence to the original DAHA for generic parameters, and thus Morita equivalences between the spherical algebras with shifted parameters. Note that the corresponding DAHAs are themselves Morita equivalent for generic parameters, since their spherical algebras are Morita equivalent.

Another feature of the double affine case is that the inverse map acts on the poset ${ }^{W} \widetilde{W}^{W}$ as a diagram automorphism (which is often trivial). In particular, if we can arrange for the pullback of the adjoint through the diagram automorphism to have isomorphic twist datum, then this gives an actual involution of the Hecke algebra, which allows us to consider self-adjoint operators (and even have a reasonable chance of proving commutativity). For a specific example of this phenomenon in the $\tilde{C}_{n}$ case, see Theorem 7.28 below.

One new phenomenon that arises in the affine case is that the group can fail to act faithfully. Since the finite Weyl group acts faithfully on $A$, the kernel is necessarily contained in the translation subgroup, and we see that there is a kernel precisely when $q$ is torsion. In that case, the action of $W \cong W \ltimes \Lambda$ on $X$ factors through a semidirect product of the form $\widetilde{W}_{q}:=$ $W \ltimes\left(\Lambda / \Lambda_{q}\right)$, where $\Lambda_{q}$ is the sublattice acting trivially (which is a multiple of either $\Lambda$ or, in the non-simply-laced case, the lattice generated by the other orbit of roots). One thus finds that $\mathcal{H}_{\widetilde{W}, \vec{T} ; \gamma}(X)$ is the sheaf algebra associated to a sheaf of algebras on the quotient $X / \widetilde{W}_{q}$. The centralizer of $k(X)$ in the generic fiber is isomorphic to $k(X)\left[\Lambda_{q}\right]_{\gamma}$, and thus the center of the generic fiber is isomorphic to $k\left(X / \widetilde{W}_{q}\right)\left[\Lambda_{q}\right]_{\gamma}^{W}$. This agrees with the generic fiber of the center, and thus the center of $\mathcal{H}_{\widetilde{W}, \vec{T} ; \gamma}(X)$ for $q$ torsion is the coordinate sheaf of an integral $X$-scheme of relative dimension $n$.

When $\vec{T}=0$, we can make this quite precise; it turns out that for $q$ torsion, $\mathcal{H}_{\widetilde{W} ; \gamma}(X)$ is an algebra à la Proposition 5.13 with $G=\widetilde{W}_{q}$ acting on an explicitly described (but no longer projective) scheme $X^{+}$; its center is thus the structure sheaf of $X^{+} / \widetilde{W}_{q}$, and everything is Noetherian. Clearly, if such a construction exists, $X^{+}$must be the relative Spec of the (abelian) restriction $\left.\mathcal{H}_{\widetilde{W} ; \gamma}(X)\right|_{\Lambda_{q}}$. This is difficult to understand directly (we will in fact give an alternate purely geometric construction and then verify that its relative coordinate ring is as described), but luckily it will largely suffice to deal with the $\tilde{A}_{1}$ case.

Thus let $C / S$ be a genus 1 curve equipped with a pair of hyperelliptic involutions $s_{0}, s_{1}$, and let $\gamma$ be a twisting datum for the corresponding action of $\tilde{A}_{1}=\left\langle s_{0}, s_{1}\right\rangle$ on $C$. We may describe $\gamma$ by giving a pair of line bundles $\mathcal{L}_{0}$ and $\mathcal{L}_{1}$ such that $\left.\gamma\right|_{\left\langle s_{i}\right\rangle}$ is the coboundary of $\mathcal{L}_{i}$. It is then straightforward to determine the residue conditions for $\mathcal{H}_{\tilde{A}_{1} ; \gamma}(C)$ when $s_{1} s_{0}$ has infinite order. We find that $c_{\left(s_{1} s_{0}\right)^{k}}$ and $c_{\left(s_{1} s_{0}\right)^{k+l} s_{1}}$ have a potential pole along $\left[C^{\left(s_{1} s_{0}\right)^{2 k+l} s_{1}}\right]$, subject to the 
condition that

$$
c_{\left(s_{1} s_{0}\right)^{k}}\left(s_{1} s_{0}\right)^{k} f+c_{\left(s_{1} s_{0}\right)^{k+l} s_{1}}\left(s_{1} s_{0}\right)^{k+l} s_{1} f
$$

is holomorphic on that reflection hypersurface for any local section

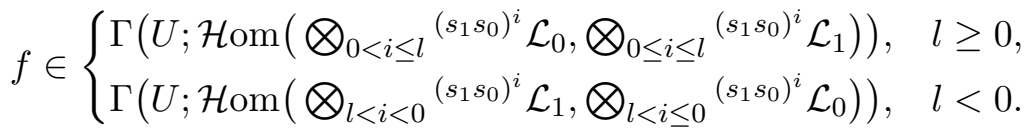

(Of course, the condition is independent of $f$ as long as $f$ is a unit in the local ring at every component of the reflection hypersurface.)

Now, suppose $s_{1} s_{0}$ has finite order $m$, so that the image of $\left\langle s_{0}, s_{1}\right\rangle$ in $\operatorname{Aut}(C)$ is actually the dihedral group of order $2 \mathrm{~m}$. Thus as automorphisms of $C$, they satisfy the braid relation $\left(s_{0} s_{1}\right)^{m / 2}=\left(s_{1} s_{0}\right)^{m / 2}$ (if $m$ is even) or $\left(s_{0} s_{1}\right)^{(m-1) / 2} s_{0}=\left(s_{1} s_{0}\right)^{(m-1) / 2} s_{1}$ (if $m$ is odd). In the dihedral group, both sides represent the longest element, and thus the corresponding Bruhat interval is the entire group. Something similar happens in $\tilde{A}_{1}$ : each side generates a Bruhat interval which is not only faithful, but a setwise section of the map to the dihedral group, and the two sections agree except on the longest element. Even better, the residue conditions in the two Bruhat intervals are very nearly the same; indeed, below the top, the conditions are necessarily the same, while the conditions involving the top element differ only mildly. We thus obtain the following, where $\pi_{q}: C \rightarrow C^{\prime}$ is the quotient by $\left\langle s_{1} s_{0}\right\rangle$ (which is translation by some torsion point $q$ ), an isogenous curve with induced hyperelliptic involution $s$ (the common action of $s_{0}$ and $s_{1}$ ). (We write $\left(s_{0} s_{1}\right)^{m / 2}$ for $\left(s_{0} s_{1}\right)^{(m-1) / 2} s_{0}$ when $m$ is odd, so that we can express the result in a uniform way for either parity.)

Proposition 6.11. Let $U \subset C^{\prime}$ be an s-invariant open subset on which there is an invertible section $g \in \Gamma\left(U ; N_{\pi_{q}}\left(\mathcal{L}_{1} \otimes \mathcal{L}_{0}^{-1}\right)^{*}\right)$. Then

$$
c_{\left(s_{0} s_{1}\right)^{m / 2}}\left(s_{0} s_{1}\right)^{m / 2}+\sum_{w<\left(s_{0} s_{1}\right)^{m / 2}} c_{w} w \in \Gamma\left(\pi_{q}^{*} U ; \mathcal{H}_{\tilde{A}_{1} ; \gamma}(C)\right)
$$

iff

$$
\pi_{q}^{*}\left(g /{ }^{s} g\right) c_{\left(s_{0} s_{1}\right)^{m / 2}}\left(s_{1} s_{0}\right)^{m / 2}+\sum_{w<\left(s_{1} s_{0}\right)^{m / 2}} c_{w} w \in \Gamma\left(\pi_{q}^{*} U ; \mathcal{H}_{\tilde{A}_{1} ; \gamma}(C)\right)
$$

Proof. This reduces to a straightforward verification that the transformation respects the residue conditions on every reflection hypersurface.

Corollary 6.12. With $U, g$ as above, let $f_{0}, f_{1} \in \Gamma\left(U ; \mathcal{O}_{X}\left(\pi_{q}^{*}\left[C^{\prime s}\right]\right)\right)$ be such that $f_{1}-\pi_{q}^{*}(g / s g) f_{0}$ is holomorphic. Then

$$
f_{0}+f_{1}\left(s_{1} s_{0}\right)^{m} \in \Gamma\left(U ; \mathcal{H}_{\tilde{A}_{1} ; \gamma}(C)\right) .
$$

Proof. Take a general section of $\mathcal{H}_{\tilde{A}_{1} ; \gamma}(C)\left[\leq\left(s_{0} s_{1}\right)^{m / 2}\right]$, apply the proposition to get a section supported on $\left[\leq\left(s_{1} s_{0}\right)^{m / 2}\right]$, take the difference, and then left-multiply by a suitable operator $h\left(s_{1} s_{0}\right)^{m / 2}$. This gives a general section $f_{0}+f_{1}\left(s_{1} s_{0}\right)^{m}$ such that $f_{1}=\pi_{q}^{*}\left(g /{ }^{s} g\right) f_{0}$, to which we may add any $f_{0}^{\prime} \in \Gamma\left(U ; \mathcal{O}_{X}\right)$.

Remark. Note that $f_{1}$ is indeed supposed to be a meromorphic section of the same bundle of which $\pi_{q}^{*}\left(g /{ }^{s} g\right)$ is a local trivialization, namely $\bigotimes_{w \in G}{ }^{w}\left(\mathcal{L}_{1} \otimes \mathcal{L}_{0}^{-1}\right)^{(-1)^{\ell(w)}}$, where $G$ is the image of $\tilde{A}_{1}$ in $\operatorname{Aut}(C)$. 
Remark. This points out that in the affine case (in contrast to the situation for more general Coxeter groups where there is no parameter $q$ ), there is something special about Bruhat intervals: the subsheaves of operators supported on more general finite subsets may fail to be flat.

The restriction of $\mathcal{O}_{C}\left[\tilde{A}_{1}\right]_{\gamma}$ to the kernel $\left\langle\left(s_{1} s_{0}\right)^{m}\right\rangle$ of the action has a natural geometric description. Indeed, it may be given in the form $\bigoplus_{j \in \mathbb{Z}} \mathcal{Z}_{\left(s_{1} s_{0}\right)^{m}}^{\otimes j}\left(s_{1} s_{0}\right)^{j m}$, which is easily recognized as the relative structure sheaf of the $\mathbb{G}_{m}$-bundle over $C$ corresponding to the invertible sheaf $\mathcal{Z}_{\left(s_{1} s_{0}\right)^{m}}$. The corollary tells us that $\mathcal{H}_{\tilde{A}_{1} ; \gamma}(C)$ contains a larger ring, which is easily recognized as coming from an affine blowup. To be precise, the restrictions of the various local sections $\pi_{q}^{*}\left(\mathrm{~g} /{ }^{s} \mathrm{~g}\right)$ to the union of reflection hypersurfaces induces a section of the $\mathbb{G}_{m}$-bundle over that union. Enlarging the algebra has the effect of blowing up that union of sections and then removing the strict transforms of the fibers over the reflection hypersurfaces.

More generally, let $\widetilde{W}$ act on $X$ with kernel $\Lambda_{q}$. The restriction of the gerbe to $\Lambda_{q}$ in particular induces a homomorphism $\Lambda_{q} \rightarrow \operatorname{Pic}(X / S)$, or equivalently a class in $H^{1}\left(X ; \operatorname{Hom}\left(\Lambda_{q}, \mathbb{G}_{m}\right)\right.$ ), and thus a principal $\operatorname{Hom}\left(\Lambda_{q}, \mathbb{G}_{m}\right)$-bundle $P$ over $X$. Each $\mathcal{Z}_{\lambda}$ for $\lambda \in \Lambda_{q}$ comes with an induced trivialization on the pullback, and this in fact trivializes that portion of the gerbe. Indeed, this compatibility is clear for any twisting datum specified as a cocycle in Cartier divisors, and (per our standing assumption) any twisting datum is fppf locally of this form. We thus obtain a natural $\widetilde{W}_{q}$-equivariant gerbe on the torus bundle, on which $\widetilde{W}_{q}$ acts faithfully, and there is a natural isomorphism $\mathcal{O}_{X}[\widetilde{W}]_{\mathcal{Z}} \cong \pi_{*} \mathcal{O}_{P}[\widetilde{W}]_{\mathcal{Z}}$.

The twisting datum itself does not directly lift, for the simple reason that $\widetilde{W}$ acts nontrivially on $\Lambda_{q}$, and thus in particular the fixed subschemes of the various reflections are codimension 2; the fixed subscheme of $r$ is a principal $\operatorname{Hom}\left(\Lambda_{q}, \mathbb{G}_{m}\right)^{\langle r\rangle}$-bundle over $\left[X^{r}\right]$. (Note that this is either a $\mathbb{G}_{m}^{n-1}$-bundle or a $\mathbb{G}_{m}^{n-1} \times \mu_{2}$-bundle, with the latter only arising when $W \ltimes \Lambda_{q} \cong W\left(\tilde{C}_{n}\right)$.) However, each reflection in $\widetilde{W}_{q}$ is the image of an infinite collection of reflections in $W \ltimes \Lambda_{q}$, the set of reflections of a copy of $\tilde{A}_{1}$. Moreover, for any reflection $r$, the subalgebra of operators supported on $\langle 1, r\rangle$ may be computed by conjugating the corresponding algebra for some simple reflection. We thus find that each such copy of $\tilde{A}_{1}$ actually gives rise to a subalgebra of the form $\mathcal{H}_{\tilde{A}_{1} ; \gamma^{\prime}}(X)$. We thus obtain additional elements as above.

We may think of each such element as a section of $\mathcal{O}_{P}\left(\left[X^{r}\right]\right)$, and find that the ideal sheaf generated by all such sections cuts out a $\operatorname{Hom}\left(\Lambda_{q}^{\langle r\rangle}, \mathbb{G}_{m}\right)$-bundle over $\left[X^{r}\right]$. (In the untwisted case, this is the subbundle of the $r$-fixed scheme containing $\left(\left[X^{r}\right], 1\right)$, and in general is uniquely determined by the requirement that it contain a section determined by the twisting datum.) Adjoining all such elements to the relative coordinate ring gives a new scheme $X^{+}$, still affine over $X$. Geometrically, $X^{+}$is obtained by blowing up the union of all such bundles then removing the strict transforms of the fibers of $P$ over the reflection hypersurfaces. This operation is $\widetilde{W}_{q^{-}}$ equivariant, and thus $X^{+}$inherits an action of $\widetilde{W}_{q}$. Now, not only does the gerbe lift, but the twisting datum as well: on $X$, it is specified by sections of line bundles on the various reflection hypersurfaces, and each reflection hypersurface of $X^{+}$lies over a reflection hypersurface of $X$, so we can simply pull back the section. (Again, any potential issues with compatibility may be reduced to the untwisted case by expressing the twisting datum via Cartier divisors, pulling back the Cartier divisors to $X^{+}$and twisting. In the untwisted case, the possible incompatibilities coming from the nontriviality of the gerbe maps go away, since the blowup makes those functions congruent to 1 on the relevant reflection hypersurface.)

We then have the following.

Theorem 6.13. Let $X^{+}$be constructed as above, with associated projection $\pi: X^{+} \rightarrow X$ and induced twisting datum $\gamma^{+}$for the action of $\widetilde{W}_{q}$ on $X^{+}$. Then there is a natural isomorphism

$$
\mathcal{H}_{\widetilde{W} ; \gamma}(X) \cong \pi_{*} \mathcal{H}_{\widetilde{W}_{q} ; \gamma^{+}}\left(X^{+}\right)
$$


Proof. The map $\mathcal{O}_{X}[\widetilde{W}]_{\gamma} \rightarrow \pi_{*} \mathcal{O}_{X+}\left[\widetilde{W}_{q}\right]_{\gamma^{+}}$extends to a map on meromorphic operators, and we find that the restriction to any rank 1 subalgebra of $\mathcal{H}_{\widetilde{W} ; \gamma}$ is contained in $\pi_{*} \mathcal{H}_{\widetilde{W}_{q} ; \gamma^{+}}\left(X^{+}\right)$. Since these generate the full algebra, we obtain a homomorphism $\mathcal{H}_{\widetilde{W} ; \gamma}(X) \rightarrow \pi_{*} \mathcal{H}_{\widetilde{W}_{q} ; \gamma^{+}}\left(X^{+}\right)$, and it remains only to show that this is surjective.

By construction, the image contains $\mathcal{O}_{X^{+}}$, and thus for any reflection $r$, the image contains any element of the form $g \pi^{*} f\left(1-\pi^{*} h r\right)$, where $g \in \Gamma\left(\pi^{*} U ; \mathcal{O}_{X^{+}}\right), f \in \Gamma\left(U ; \mathcal{O}_{X}\left(\left[X^{r}\right]\right)\right)$, and $h \in$ $\Gamma\left(U ; \mathcal{Z}_{r}^{*}\right)$ restricts to give the twisting datum along $r$. For $U$ sufficiently small (but containing any chosen codimension 1 point), the elements of the form $g \pi^{*} f \operatorname{span} \Gamma\left(U ; \mathcal{O}_{X}\left(\left[\left(X^{+}\right)^{r}\right]\right)\right)$, and thus we obtain any element in $\Gamma\left(U ; \mathcal{O}_{X^{+}}\right)+\Gamma\left(U ; \mathcal{O}_{X^{+}}\left(\left[X^{r}\right]\right)\right)\left(1-\pi^{*} h r\right)$. But this is precisely the algebra corresponding to $\langle 1, r\rangle$ in $\mathcal{H}_{\widetilde{W}_{q} ; \gamma^{+}}\left(X^{+}\right)$, and these subalgebras again generate the full algebra.

Corollary 6.14. The center of $\mathcal{H}_{\widetilde{W} ; \gamma}(X)$ as a sheaf of algebras over $X / \widetilde{W}_{q}$ is naturally isomorphic to the relative coordinate ring of $X^{+} / \widetilde{W}_{q}$.

Proof. Since $\widetilde{W}_{q}$ acts faithfully on $X^{+}$, the centralizer of $\mathcal{O}_{X^{+}}$in $\mathcal{H}_{\widetilde{W}_{q}, \gamma^{+}}\left(X^{+}\right)$is precisely $\mathcal{O}_{X^{+}}$; furthermore, for an element of $\mathcal{O}_{X^{+}}$to commute with sections of $\mathcal{Z}_{g} g$ for all $g \in \widetilde{W}_{q}$, it must be $\widetilde{W}_{q^{-}}$-invariant. We thus find that the center is contained in $\mathcal{O}_{X^{+}}^{\widetilde{W}_{q}}=\mathcal{O}_{X^{+}} / \widetilde{W}_{q}$. This subalgebra is clearly central, and thus the claim holds.

We would also, of course, like to understand the center of the spherical algebra. When $X \rightarrow X / W$ is flat, a symmetric idempotent already establishes a Morita equivalence between $\mathcal{H}_{W}(X)$ and $\mathcal{O}_{X / W}$, and thus forces the center of the spherical algebra to agree with that of the DAHA itself. Of course, as we noted above, this map is essentially never flat (with the notable exceptions of the action of $W\left(A_{n}\right)$ on the sum zero subscheme and the action of $W\left(C_{n}\right)$ on $E^{n}$ by signed permutations), but it turns out to be close enough to let us prove the analogous result.

Proposition 6.15. Suppose $\gamma$ is trivial on $W$ and the root kernel of $W$ is diagonalizable. Then there is a natural isomorphism $Z\left(\mathcal{H}_{\widetilde{W} ; \gamma}(X)\right) \cong Z\left(\mathcal{H}_{\widetilde{W}, W ; \gamma}(X)\right)$, and both algebras are strongly flat.

Proof. Let $U$ be the largest $\widetilde{W}_{q}$-invariant open subset of $X$ such that every point of $U$ lies over a regular point of $X / W$. The morphism $U \rightarrow U / W$ is a morphism of regular schemes with 0-dimensional fibers, so is flat, and thus in particular $\pi_{W *} \mathcal{O}_{U}$ is locally free. It follows that a covering of $U$ by symmetric idempotents induces a Morita equivalence between $\mathcal{H}_{W}(U)$ and $\mathcal{O}_{U / W}$, and thus between the restrictions of $\mathcal{H}_{\widetilde{W} ; \gamma}(X)$ and $\mathcal{H}_{\widetilde{W}, W ; \gamma}(X)$ (viewed as sheaf algebras on $\left.X / \widetilde{W}_{q}\right)$ to $U / \widetilde{W}_{q}$. In particular, we find that the natural morphism between the centers is an isomorphism on $U / \widetilde{W}_{q}$.

For any other $\widetilde{W}_{q}$-invariant open subset $V$, given any element $\mathcal{D} \in \Gamma\left(V ; Z\left(\mathcal{H}_{\widetilde{W}, W ; \gamma}(X)\right)\right)$, we may restrict it to $V \cap U$ and transport it through the isomorphism of centers to obtain an element $\mathcal{D}^{\prime} \in \Gamma\left(V \cap U ; Z\left(\mathcal{H}_{\widetilde{W} ; \gamma}(X)\right)\right)$. By normality of $X / W, U$ contains every codimension 1 point of $X$, and thus $\Gamma\left(V \cap U ; \mathcal{H}_{\widetilde{W} ; \gamma}(X)\right)=\Gamma\left(V ; \mathcal{H}_{\widetilde{W} ; \gamma}(X)\right)$, so that $\mathcal{D}^{\prime}$ is actually holomorphic on $V$ as a section of the DAHA. Since an operator is in the center of the DAHA iff it is in the center of the meromorphic twisted group algebra, we conclude that $\mathcal{D}^{\prime} \in \Gamma\left(V ; Z\left(\mathcal{H}_{\widetilde{W} ; \gamma}(X)\right)\right)$, and thus the natural map from the center of the DAHA to the center of the spherical algebra is indeed surjective.

Corollary 6.16. If $\Lambda$ acts trivially on $X, \gamma$ is trivial on $W$, and the root kernel of $W$ is diagonalizable, then there is a natural isomorphism $Z\left(\mathcal{H}_{\widetilde{W} ; \gamma}(X)\right) \cong \mathcal{H}_{\widetilde{W}, W ; \gamma}(X)$. 
Remark. More generally, if $\gamma=\gamma^{\prime} \partial \mathcal{L}$ with $\gamma^{\prime}$ trivial on $W$, then we can identify $Z\left(\mathcal{H}_{\widetilde{W} ; \gamma}(X)\right)$ with the twist of $\mathcal{H}_{\widetilde{W}, W ; \gamma^{\prime}}(X)$ by $\mathcal{L}$. In particular, if the root kernel is trivial, we can always do this fppf locally on $S$.

Remark. One consequence is that there is a natural Poisson structure on $X^{+} / \widetilde{W}_{q}$, since as a spherical algebra, its relative coordinate ring is the commutative fiber of a family of noncommutative algebras. Presumably this Poisson structure has a natural description in terms of the geometry of $X^{+}$. Note that $\mathcal{O}_{X^{+}}$is an elliptic analogue of the subalgebra of the usual DAHA generated by the commutative subalgebras, so it would not be unreasonable to expect it to have a flat deformation to non-torsion $q$, corresponding to a pulled back Poisson structure on $X^{+}$. This fails, however: the pullback of the Poisson structure on $\mathcal{O}_{X^{+}}^{\widetilde{W}_{q}}$ is only meromorphic on $\mathcal{O}_{X^{+}}$. Indeed, it agrees with the natural Poisson bracked on $\mathcal{O}_{X}[\Lambda]_{\gamma}$, but the bracket of one of the additional generators with a function with nonzero partial derivative on the reflection hypersurface will fail to be holomorphic. Note that $\widetilde{W}_{q}$-invariance forces this to vanish, so the restriction of this meromorphic Poisson structure to the $\widetilde{W}_{q}$-invariants is holomorphic.

Unfortunately, the trick with $\tilde{A}_{1}$-subalgebras breaks down completely when the system of parameters is nontrivial. As a result, we cannot give such a simple description of the center. It turns out, however, that we can reduce to the case $q=0$.

Return for the moment to the case $\vec{T}=0$. The sublattice $\Lambda_{q}$ is isomorphic as a group with $W$ action to one of the root lattices of $W$, and thus the semidirect product $W \ltimes \Lambda_{q}$ is itself an affine Weyl group. (Note that when $\widetilde{W}$ is of type $B$ or $C$, this new affine Weyl group may be of the other type.) The quotient $\Lambda / \Lambda_{q}$ acts on both $X$ and $X^{+}$, and we observe that $X^{+} /\left(\Lambda / \Lambda_{q}\right)$ is generically a torus bundle over $Y=X /\left(\Lambda / \Lambda_{q}\right)$, and is more precisely an affine blowup of $Y$ in the corresponding bundles over the reflection hypersurfaces. We thus obtain the following.

Proposition 6.17. There is an induced twisting datum $N_{X / Y}(\gamma)$ on $Y$ such that the center of $\mathcal{H}_{\widetilde{W} ; \gamma}(X)$ is canonically isomorphic to the center of $\mathcal{H}_{W \ltimes \Lambda_{q} ; N_{X / Y}(\gamma)}(Y)$.

Proof. When $\gamma$ is trivial, this holds with $N_{X / Y}(\gamma)$ trivial. More generally, if $\gamma$ is represented by a cocycle in Cartier divisors, we may obtain $N_{X / Y}(\gamma)$ by taking the images of the different Cartier divisors, and the resulting twisting datum is independent of the choice of cocycle representation.

We can then say the following in general, where $N_{X / Y} \vec{T}$ is again obtained by taking the image of each $T_{\alpha}$ under the induced isogeny of coroot curves.

Theorem 6.18. If the root kernel of $W$ is diagonalizable, then the center of $\mathcal{H}_{\widetilde{W} ; \vec{T} ; \gamma}(X)$ is canonically isomorphic to the center of $\mathcal{H}_{W \ltimes \Lambda_{q} ; N_{X / Y} \vec{T} ; N_{X / Y} \gamma}(Y)$.

Proof. The claim respects twisting by line bundles, so we may assume that $\gamma$ is trivial on $W$, and replace the algebra on $Y$ with the spherical algebra $\mathcal{H}_{W \ltimes \Lambda_{q}, W ; N_{X / Y} \vec{T} ; N_{X / Y} \gamma}(Y)$. (Both centers will then be isomorphic to the same spherical algebra.)

Imposing a system of parameters does not change the generic fiber, and thus we have

$$
Z\left(\mathcal{H}_{\widetilde{W} ; \vec{T} ; \gamma}(X)\right)=\mathcal{H}_{\widetilde{W} ; \vec{T} ; \gamma}(X) \cap Z\left(\mathcal{H}_{\widetilde{W} ; \gamma}(X)\right)
$$

When $\vec{T}$ is sufficiently general, we may apply Corollary 6.4 to identify the precise conditions for an element of $Z\left(\mathcal{H}_{\widetilde{W} ; \gamma}(X)\right)$ to be contained in the smaller Hecke algebra. (Note that determining whether a negative root of $\widetilde{W}$ becomes positive under a translation is quite straightforward.) In particular, we find as in the discussion above regarding reflection hypersurfaces that the divisor along which each coefficient must vanish is $\Lambda / \Lambda_{q}$-invariant. (To be precise, it is a nonnegative 
linear combination of sums of the form $\sum_{x \in\left\langle q_{\alpha}\right\rangle}{ }^{x} T_{\alpha}$, where $q_{\alpha}$ generates the group of translations acting on the corresponding coroot curve.) Thus imposing the condition that the coefficient is $\Lambda / \Lambda_{q}$-invariant has no additional effect on the vanishing condition, so that the algebras agree under the given constraint on $\vec{T}$.

Since the spherical algebra is strongly flat, any section for special $\vec{T}$ extends to a neighborhood in parameter space. The corresponding operator in $Z\left(\mathcal{H}_{\widetilde{W} ; \gamma}(X)\right)$ is thus contained in $\mathcal{H}_{\widetilde{W} ; \vec{T} ; \gamma}(X)$ for generic parameters, and thus for all parameters. It follows in particular that the image of the spherical algebra saturates the Bruhat filtration, and is thus surjective as required.

Remark. In the $\vec{T}=0$ case, we were able to use $\mathcal{O}_{X^{+}}$as an intermediate step to understanding the center. Is there an analogous geometric description of $\mathcal{O}_{X^{+}} \cap \mathcal{H}_{\widetilde{W}_{;} ; \vec{T}_{;}}(X)$ ? For $\vec{T}$ transverse to reflection hypersurfaces, we can again describe this intersection via vanishing conditions, but it does not follow from the above discussion that the intersection is flat, and the obvious comparison to the spherical module fails, as the latter is not $\widetilde{W}_{q}$-invariant. It is likely that a description in the $\tilde{A}_{1}$ case could be extended to general type.

The same argument gives the following.

Corollary 6.19. If the root kernel of $W$ is diagonalizable and $\gamma$ is trivial on $W$, then the center of $\mathcal{H}_{\widetilde{W} ; \vec{T} ; \gamma}(X)$ is canonically isomorphic to the center of $\mathcal{H}_{\widetilde{W}, W ; \vec{T} ; \gamma}(X)$.

Note that $Z\left(\mathcal{H}_{\widetilde{W} ; \vec{T} ; \gamma}(X)\right)$ is always a domain (it is contained in the structure sheaf of the integral scheme $\left.X^{+}\right)$. We conjecture that it is also Noetherian, and moreover that $\mathcal{H}_{\widetilde{W} ; \vec{T} ; \gamma}(X)$ is finite over its center, just as in the $\vec{T}=0$ case. Assuming the technique of [1] (reducing to finite fields, where $q$ is always torsion) could be adapted to the case of sheaf algebras, this would be enough to prove $\mathcal{H}_{\widetilde{W} ; \vec{T} ; \gamma}(X)$ Noetherian even when $q$ was not torsion.

The above description of the DAHA for $q$ torsion can in principle be used to give explicit degenerations of the residue conditions, though in practice it seems simpler to check the analogous conditions on $\mathcal{H}_{\widetilde{W}_{q} ; \gamma^{+}}\left(X^{+}\right)$. For that, the following general reduction (which we implicitly used above) may be useful. Recall that any reflection $r \in R(W)$ induces a corresponding subgroup of type $\tilde{A}_{1}$ of $\widetilde{W}$ (generated by the reflections in $r \Lambda$ ), and that this gives rise to a corresponding subalgebra which we denote by $\mathcal{H}_{\tilde{A}_{1}(r) ; \gamma}(X)$.

Proposition 6.20. An element $\sum_{w} c_{w} w \in k(X)[\widetilde{W}]_{\gamma}$ is a local section of $\mathcal{H}_{\widetilde{W}}(X)$ iff its coefficients are holomorphic away from the reflection hypersurfaces and for any reflection $r \in R(W)$ and any $w_{0} \in W$, the operator $\sum_{w \in \tilde{A}_{1}(r) w_{0}} c_{w} w$ is a section of the localization of $\mathcal{H}_{\tilde{A}_{1}(r) ; \gamma}(X) \mathcal{Z}_{w_{0}} w_{0}$ to the corresponding union of reflection hypersurfaces.

Proof. An element is in the DAHA if it is holomorphic away from the reflection hypersurfaces and is in the DAHA over the localization to the union of reflection hypersurfaces. Since the reflection hypersurfaces of $\tilde{A}_{1}(r)$ for distinct $r$ remain transverse to each other even after specializing $q$, the corresponding conditions are independent. The conditions along the reflection hypersurfaces of $\tilde{A}_{1}(r)$ for special $q$ are the limit from the case for general $q$, where they agree with the residue conditions on $\mathcal{H}_{\tilde{A}_{1}(r) ; \gamma}(X)$. That algebra is itself a DAHA, and thus the corresponding conditions remain flat for special $q$ as well.

One possible application of this reduction is to the construction of degenerations: a limit of $\mathcal{H}_{\widetilde{W} ; \gamma}(X)$ should be well-behaved as long as the limits of the various $\mathcal{H}_{\tilde{A}_{1}(r) ; \gamma}(X)$ are wellbehaved and there is no further coalescence of reflection hypersurfaces. (There are, however, technical issues, in that most natural degenerations will break the normality of $X$, but one may be able to finesse this by working over $X / W$ instead, as the quotient will tend to remain a weighted projective space.) 
One such limit of interest is the other natural $q \rightarrow 0$ (or $q$ torsion) limit: rather than take the limit to a twisted group algebra in which the group does not act faithfully, one might instead take a limit to an algebra of differential-reflection operators; i.e., take the limit in such a way as to consider how the operators themselves actually act. This would presumably be the correct way to interpret the algebra of holomorphy-preserving operators if one does not suppress the poles for $q$ torsion, but is not directly accessible via our techniques. In particular, the resulting algebra would not be generated by the rank 1 subalgebras, and the corresponding spherical algebra is almost certainly not a domain when $q$ is a nontrivial torsion element.

\section{The $C^{\vee} C_{n}$ case}

We now restrict our attention to the case that the affine Weyl group is of type $C$. This has a natural action on the family $\mathcal{E}^{n+1}$ given as follows:

$$
\begin{aligned}
& s_{0}\left(z_{1}, \ldots, z_{n}, q / 2\right)=\left(q-z_{1}, z_{2}, \ldots, z_{n}, q / 2\right), \\
& s_{n}\left(z_{1}, \ldots, z_{n}, q / 2\right)=\left(z_{1}, \ldots, z_{n-1},-z_{n}, q / 2\right),
\end{aligned}
$$

while for $1 \leq i \leq n-1, s_{i}$ swaps $z_{i}$ and $z_{i+1}$. Here we denote the last coordinate in $\mathcal{E}^{n+1}$ by $q / 2$, so that $q$ is twice that coordinate. We use this notation since the corresponding family of actions of $\tilde{C}_{n}$ on $\mathcal{E}^{n}$ (and thus the resulting Hecke algebras) depends only on $q$, but it will be convenient (and more symmetric) to be able to divide $q$ by 2 . We find for this action that the simple coroot morphisms are $q / 2-z_{1}, z_{1}-z_{2}, \ldots, z_{n-1}-z_{n}, z_{n}$, and thus that this is in fact an action of coroot type, as required for our theory. We also have an action corresponding to the diagram automorphism:

$$
\omega\left(z_{1}, \ldots, z_{n}\right)=\left(q / 2-z_{n}, \ldots, q / 2-z_{1}\right)
$$

which clearly permutes the simple coroot morphisms as expected.

The root curves are all isomorphic to $\mathcal{E}$, and the simple root morphisms are given by

$$
(-1,0, \ldots, 0),(1,-1,0, \ldots, 0),(0,1,-1,0, \ldots, 0), \ldots,(0, \ldots, 0,1,-1,0),(0, \ldots, 0,1,0) .
$$

It follows that the root kernel for any finite parabolic subgroup is trivial, so that there will be no difficulties with invariants and (local) idempotents.

In fact, we have the following.

Proposition 7.1. For any finite parabolic subgroup $W_{I} \subset \tilde{C}_{n}$, the quotient $\mathcal{E}^{n} / W_{I}$ is smooth over $\mathcal{M}_{1,1}$.

Proof. Any quotient $\mathcal{E}^{n} / W_{I}$ is a product of symmetric powers of $\mathcal{E}$ and a quotient or two of the form $\mathcal{E}^{m} / C_{m}$ (using the diagram automorphism to identify parabolic subgroups involving $s_{0}$ with the usual hyperoctahedral group). Symmetric powers of a curve are smooth, so there is no difficulty. For the quotient by the full hyperoctahedral group, we first observe that the quotient by the normal subgroup of order $2^{m}$ is just the product of $n$ copies of the quotient of $\mathcal{E}$ by $[-1]$, a.k.a. $\mathbb{P}^{1}$. We thus find that $\mathcal{E}^{m} / C_{m} \cong\left(\mathbb{P}^{1}\right)^{m} / S_{m} \cong \mathbb{P}^{m}$, giving smoothness as required.

"Miracle flatness" immediately gives the following, which in particular ensures that the spherical algebras we consider will be locally free in a suitable sense (i.e., that their direct images in either copy of $\mathcal{E}^{n} / W$ are locally free).

Corollary 7.2. For any parabolic subgroup $W_{I} \subset C_{n}$, the quotient maps $\mathcal{E}^{n} \rightarrow \mathcal{E}^{n} / W_{I}$ and $\mathcal{E}^{n} / W_{I} \rightarrow \mathcal{E}^{n} / C_{n}$ are flat. 
Suppose for the moment that $\vec{T}_{0}={ }^{\omega} \vec{T}_{n}$. Then we may consider the extended double affine Hecke algebra obtained by adjoining $\mathcal{O}_{X} \omega$ to $\mathcal{H}_{\tilde{C}_{n} ; \vec{T}}\left(\mathcal{E}^{n}\right)$. This has a natural $\mathbb{Z} / 2 \mathbb{Z}$-grading, and it will be useful to think of it as corresponding to a (sheaf) category with two objects rather than a sheaf algebra. That is, we have objects 0 and 1 with endomorphisms given by the sheaf algebra $\mathcal{H}_{\tilde{C}_{n}: \vec{T}}\left(\mathcal{E}^{n}\right)$ and the remaining morphisms given in either direction by the sheaf bimodule $\mathcal{H}_{\tilde{C}_{n} ; \vec{T}}\left(\mathcal{E}^{n}\right) \omega$. Doing this actually lets us remove the constraint on the parameters: we may always obtain a sheaf category with two objects by taking

$$
\begin{array}{ll}
\operatorname{Hom}(0,0)=\mathcal{H}_{\tilde{C}_{n} ; \vec{T}}\left(\mathcal{E}^{n}\right), & \operatorname{Hom}(0,1)=\mathcal{H}_{\tilde{C}_{n} ; \omega \vec{T}}\left(\mathcal{E}^{n}\right) \omega, \\
\operatorname{Hom}(1,0)=\mathcal{H}_{\tilde{C}_{n} ; \vec{T}}\left(\mathcal{E}^{n}\right) \omega, & \operatorname{Hom}(1,1)=\mathcal{H}_{\tilde{C}_{n} ; \omega \vec{T}}\left(\mathcal{E}^{n}\right),
\end{array}
$$

where $\mathcal{H}_{\tilde{C}_{n} ; \vec{T}}\left(\mathcal{E}^{n}\right) \omega$ denotes the bimodule obtained by twisting the regular bimodule of the DAHA by the isomorphism induced by $\omega$.

The Bruhat order on the extended affine Weyl group induces a Bruhat filtration on this category (which agrees with the usual Bruhat filtration coming from viewing each Hom bimodule as a regular module of some DAHA). Although of course this filtration is useful in itself, we will also make great use of a filtration obtained from a much coarser order. The category is certainly generated by the rank 1 subalgebras along with $\omega$, but since $\omega$ permutes the rank 1 subalgebras, we can actually omit the subalgebras corresponding to $s_{0}$ from the generators. As a result, we find that the category is generated by the two morphisms corresponding to $\omega$ along with the Hecke algebras in each degree corresponding to the finite Weyl group. We may then define a filtration on each Hom bimodule by the number of times $\omega$ was used; e.g., the degree $\leq d$ piece of $\operatorname{Hom}(0, d \bmod 2)$ is the image of

$$
\mathcal{H}_{C_{n} ; \vec{T}}(X) \omega \mathcal{H}_{C_{n} ; \omega \vec{T}}(X) \omega \mathcal{H}_{C_{n} ; \vec{T}}(X) \cdots
$$

(with $d$ copies of $\omega$ and $d+1$ finite Hecke algebras as tensor factors). We can express each finite Hecke algebra factor as the image of a product of rank 1 algebras (corresponding to the longest element of $C_{n}$ ), move all of the copies of $\omega$ to the end, and then use Corollary 5.20 to express this in terms of a Bruhat interval in the appropriate Hecke algebra. Moreover, that Bruhat interval is clearly a union of $(W, W)$ double cosets, so is determined by the corresponding set of dominant weights; we find that the condition to be degree $\leq d$ is simply that the first coefficient of the dominant weight is $\leq d / 2$.

This leads to a "graded" (or "compactified") version of the extended DAHA: take the sheaf category with objects $\mathbb{Z}$ generated by elements $\omega \in \operatorname{Hom}(k, k+1)$ and algebras $\mathcal{H}_{C_{n} ; \vec{T}}(X) \subset$ $\operatorname{Hom}(2 k, 2 k), \mathcal{H}_{C_{n} ; \omega \vec{T}}(X) \subset \operatorname{Hom}(2 k+1,2 k+1)$. (We may think of this as a sort of Rees algebra corresponding to the filtration, taking into account parity.)

Note that when asking whether two (small) sheaf categories are isomorphic, there are actually two natural notions. The issue here is that for any automorphism of an object of a category, there is a corresponding inner automorphism of the category as a whole (and more generally for any assignment of an automorphism to each object). In our case, any unit on parameter space gives such an inner automorphism, and since we are primarily interested in the individual fibers, we should extend that to allow local units. This leads to a notion of twisting objects by line bundles on the base; note that the resulting sheaf category will still be locally isomorphic to the original sheaf category. In general, we will often only state that given sheaf categories are isomorphic locally on the base; this is mainly to save the bookkeeping effort of determining precisely which line bundles one needs to twist by to make the isomorphism global. In particular, when specifying line bundles and equivariant gerbes, the $z$-independent terms of the polarization and weight will be largely irrelevant, so we can make the simplest consistent choice without having to worry too much about which choice would make later polarizations simpler. 
Of course, we have yet to incorporate a twisting datum. We first note that the underlying equivariant gerbe induces a cocycle valued in pairs of polarizations and weights. We can embed the $\tilde{C}_{n}$-module of polarizations in the degree 2 subspace of $(1 / q) \mathbb{Q}[\vec{z}, q, \vec{\pi}]$ (where $\vec{\pi}$ corresponds to additional factors of $\mathcal{E}$ which we include to allow some room for further parameters), and similarly for the $\tilde{C}_{n}$-module of weights. Since $q$ is $\tilde{C}_{n}$-invariant, both rational $\tilde{C}_{n}$-modules are isomorphic to the corresponding module $\mathbb{Q}[\vec{z}, \vec{\pi}]$ obtained by specializing $q=1$. We can compute $H^{1}$ of this module by restriction to the (finite index) translation subgroup, where the filtration by degree makes it easy to verify that $H^{1}$ is trivial. It follows that the given cocycle is the coboundary of a pair $\left(p_{3}(\vec{z}, q, \vec{\pi}) / q, p_{1}(\vec{z}, q, \vec{\pi}) / q\right)$, where $p_{3}$ and $p_{1}$ are homogeneous polynomials of degree 3 and 1 respectively. Of course, $p_{3}$ and $p_{1}$ are only determined modulo 0 -cocycles, but those are again easily seen to be just the polynomials independent of $\vec{z}$.

In our case, since our primary interest is in the spherical algebras, we want the twisting datum to be trivial on $C_{n}$. The same must in particular hold for the equivariant gerbe, so that $p_{3}$ and $p_{1}$ must be $C_{n}$-invariant polynomials. (More precisely, they must be $C_{n}$-invariant modulo 0 -cocycles, but we can then average over $C_{n}$ without changing the coboundary.) Since we are allowed to ignore terms independent of $\vec{z}$, we see that we may as well take $p_{1}=0$ and $p_{3}=$ $\frac{\lambda(q / 2, \vec{\pi})}{q} \sum_{i} \frac{z_{i}^{2}}{2}$ for some linear polynomial $\lambda$ with rational coefficients. Imposing the condition that the coboundary consist of actual polarizations then forces $\lambda$ to have integer coefficients. That is, the value of the coboundary at $s_{0}$ is $p_{3}(\vec{z})-{ }^{s_{0}} p_{3}(\vec{z})=\lambda(q / 2, \vec{\pi}) z_{1}-\lambda(q / 2, \vec{\pi}) \frac{q}{2}$, which is integral iff $\lambda$ is integral since $q / 2$ is a variable.

To extend this to a twisting datum, it suffices to choose a meromorphic section of the restriction to $\left\langle s_{0}\right\rangle$ and represent the corresponding Cartier divisor as $D_{0}-{ }^{s_{0}} D_{0}$ with $D_{0}$ transverse to $\left[X^{s_{0}}\right]$. Since $D_{0}$ is only determined modulo $s_{0}$-invariant divisors and linear equivalence, it is equivalent to specify the polarization

$$
k \frac{z_{1}\left(z_{1}-q\right)}{2}+\frac{\lambda(q / 2, \vec{\pi}) z_{1}}{2}
$$

with $k \in\{0,1\}$. (Again, the constant term is irrelevant for our purposes.) Note that this imposes a stronger integrality constraint on $\lambda$, which must now have even coefficients.

Of course, we saw above when discussing the elliptic Gamma "function" that not every suitably integral cocycle has meromorphic sections, even for the translation subgroup. Luckily, in our case, we can easily write down explicit products of elliptic Gamma functions that do the trick. To be precise, consider the product

$$
\prod_{1 \leq i \leq n} \Gamma_{q}\left(a \pm z_{i}\right)
$$

where $a$ is linear in $q / 2$ and $\pi$. Here and below, we have used the shorthand notation that multiple arguments to $\Gamma_{q}$ or $\vartheta$ represent a product and the appearance of \pm in the argument means that both signs should be used; thus $\Gamma_{q}\left(a \pm z_{i}\right)=\Gamma_{q}\left(a+z_{i}\right) \Gamma_{q}\left(a-z_{i}\right)$.

This is $C_{n}$-invariant, and has polarization (ignoring the $z$-independent term)

$$
(2 a-q) \sum_{i} \frac{z_{i}^{2}}{2} .
$$

Moreover, we find

$$
\frac{{ }^{s_{0}} \prod_{1 \leq i \leq n} \Gamma_{q}\left(a \pm z_{i}\right)}{\prod_{1 \leq i \leq n} \Gamma_{q}\left(a \pm z_{i}\right)}=\frac{\vartheta\left(a-z_{1}\right)}{\vartheta\left(a-q+z_{1}\right)},
$$

and thus this corresponds to the twisting datum with $D_{0}=\left[z_{1}=a\right]$. This gives the general degree 1 case, and we may obtain the general degree 0 case by taking a ratio of two such products. 
Such a choice of product induces an embedding of the twisted Hecke algebra in the algebra of meromorphic difference-reflection operators; equivalently, meromorphic sections of the twisted Hecke algebra act on formal functions of the form $f \gamma$, where $\gamma$ is the product of $\Gamma_{q}$ symbols. This lets us extend the holomorphy-preserving property to the twisted case: if $f$ is locally holomorphic away from the poles of the sections of the cocycle corresponding to $\gamma$, then so its image. This leads to issues where $\gamma$ has poles, but we have enough choice in how we represent things that the corresponding invariant localizations give a finite covering. (I.e., in the degree 0 case, we may take $\gamma=\prod_{1 \leq i \leq n} \Gamma_{q}\left(v+a \pm z_{i}\right) / \Gamma_{q}\left(v \pm z_{i}\right)$ with $v$ varying; in degree 1 , we take $\gamma=\prod_{1 \leq i \leq n} \Gamma_{q}\left(v \pm z_{i}, w \pm z_{i}\right) / \Gamma_{q}\left(v+w-a \pm z_{i}\right)$ with $v, w$ varying. $)$

To include the diagram automorphism, we note that because we extended to a category above, we may choose a different product of $\Gamma_{q}$ symbols for each object, and need only have a line bundle $\mathcal{L}_{i}$ in each degree such that sections of $\mathcal{L}_{i} \omega$ take $k(X) \gamma_{i}$ to $k(X) \gamma_{i+1}$. This is easy enough, since

$$
\begin{aligned}
& \frac{{ }^{\omega} \prod_{1 \leq i \leq n} \Gamma_{q}\left(a \pm z_{i}\right)}{\prod_{1 \leq i \leq n} \Gamma_{q}\left(a-q / 2 \pm z_{i}\right)}=\prod_{1 \leq i \leq n} \vartheta\left(a-q / 2-z_{i}\right), \\
& \frac{{ }^{\omega} \prod_{1 \leq i \leq n} \Gamma_{q}\left(a \pm z_{i}\right)}{\prod_{1 \leq i \leq n} \Gamma_{q}\left(a+q / 2 \pm z_{i}\right)}=\prod_{1 \leq i \leq n} \frac{1}{\vartheta\left(a-q / 2+z_{i}\right)} .
\end{aligned}
$$

In the degree 0 case, this ends up not changing the twisting datum, but in degree 1 , the polarization of $\mathcal{L}_{i}$ ends up alternating between positive and negative. This would increase the number of cases we would need to consider, so it will be convenient to enlarge the category even further by replacing each object by a sequence of objects, one for each (isomorphism class of) line bundle on $\mathbb{P}^{n}$. The Hom bimodule between two objects in the enlarged category is then just the twist of the original Hom bimodule by the pair of line bundles (inverting the one on the domain side). The benefit of this is that we can move between the two degree 1 cases by twisting by $\mathcal{O}_{\mathbb{P}^{n}}( \pm 1)$, and thus in the enlarged category, there is only one case.

It turns out that even the above category is not quite general enough to include everything we want to do for the spherical algebras. As a result, we will first focus our attention on the case in which the endpoints do not have any parameters assigned. We also specialize to the usual Macdonald-ish case, in which $T_{i}$ for $1 \leq i \leq n-1$ is the divisor $t+z_{i}-z_{i+1}=0$. (The definitions work more generally; in particular, we note that everything works mutatis mutandum for the case $T_{i}=0$ for $1 \leq i \leq n-1$, though oddly enough this " $t$-free" case turns out to be harder to understand in a number of respects.) It also turns out that most of the symmetries of the algebra do not preserve the untwisted case, but do preserve a particular twist; this leads us to make a somewhat odd-appearing choice in parametrizing twists. The specific basis we use for the $\mathbb{Z}^{2}$ of objects is inspired by [26] (where it in turn came from the geometry of rational surfaces); this also informs the choice of parameters (in [26], there was a parameter $\eta$ which we have arranged to be 0 ).

Definition 7.3. The even elliptic DAHA $\mathcal{H}_{\eta^{\prime} ; q, t}^{(n)}$ (of type $C$ ) is the smallest sheaf category on $\mathcal{E}^{n+3}$ with objects $\mathbb{Z}\langle s, f\rangle$ such that

$$
\mathcal{H}_{\eta^{\prime} ; q, t}^{(n)}\left(d s+d_{1}^{\prime} f, d s+d_{2}^{\prime} f\right)=\mathcal{O}_{\mathbb{P}^{n}}\left(d_{2}^{\prime}\right) \otimes \mathcal{H}_{C_{n} ; \vec{T}_{t}}\left(\mathcal{E}^{n+3}\right) \otimes \mathcal{O}_{\mathbb{P}^{n}}\left(-d_{1}^{\prime}\right)
$$

and

$$
\mathcal{H}_{\eta^{\prime} ; q, t}^{(n)}\left(d s+d_{1}^{\prime} f,(d+1) s+d_{2}^{\prime} f\right) \supset \mathcal{L} \omega,
$$

where $\mathcal{L}$ is the line bundle with polarization

$$
-\left(d_{1}^{\prime}-d_{2}^{\prime}+1\right) \sum_{i} z_{i}^{2}-\left((n-1) t+\eta^{\prime}+\left(d-d_{1}^{\prime}+1\right) q\right) \sum_{i} z_{i}+\left(d-d_{1}^{\prime}\right) q^{2} / 4 .
$$


The odd elliptic DAHA $\mathcal{H}_{x_{0} ; q, t}^{\prime(n)}$ is defined similarly, except that $\mathcal{L}$ has polarization

$$
-\left(2 d_{1}^{\prime}-2 d_{2}^{\prime}+3\right) \sum_{i} \frac{z_{i}^{2}}{2}-\left((n-1) t+x_{0}+\left(3 d-2 d_{1}^{\prime}+2\right) q / 2\right) \sum_{i} z_{i}+\left(3 d-2 d_{1}^{\prime}\right) q^{2} / 8 .
$$

Remark. In this case, we were able to choose the $z$-independent part of the polarization to make everything globally consistent, where $\mathcal{O}_{\mathbb{P}^{n}}(1)$ is chosen so as to pull back to the line bundle with polarization $\sum_{i} z_{i}^{2}$. We can recover the usual (compactified) elliptic DAHA by restricting the algebra $\mathcal{H}_{-(n-1) t-q ; q, t}^{(n)}$ to the subset $\mathbb{Z}(s+f)$ of objects; if we restrict to even multiples and then invert the sections $1 \in \operatorname{Hom}(d(s+f),(d+2)(s+f))$, we recover the uncompactified elliptic DAHA. We should note that while $\mathcal{H}_{\eta^{\prime} ; q, t}^{(n)}(0,2 s+2 f)$ always contains 1 , this is only true locally on the base for $\mathcal{H}_{x_{0} ; q, t}^{\prime(n)}(0,2 s+3 f)$, and it is not possible to fix this without also changing the particular representative of $\mathcal{O}_{\mathbb{P}^{n}}(1)$.

We similarly let $\mathcal{S}_{\eta^{\prime} ; q, t}^{(n)}$ and $\mathcal{S}_{x_{0} ; q, t}^{(n)}$ denote the corresponding spherical categories (i.e., replacing each Hom bimodule by the appropriate subquotient). Each Hom bimodule in one of these categories is a sheaf bimodule on the quotient $\mathbb{P}^{n}=\mathcal{E}^{n} / C_{n}$, every local section of which is a meromorphic difference operator on $\mathcal{E}^{n}$.

Proposition 7.4. The subsheaf corresponding to any Bruhat order ideal in either $\mathcal{S}_{\eta^{\prime} ; q, t}^{(n)}$ or $\mathcal{S}_{x_{0} ; q, t}^{(n)}$ is a coherent sheaf bimodule on $\mathbb{P}^{n} \times \mathcal{E}^{3} \mathbb{P}^{n}$, and the direct image in either $\mathbb{P}^{n}$ is locally free.

Proof. This reduces to the corresponding statement for the subquotients in the Bruhat filtration, each of which comes from a line bundle on the quotient by a parabolic subgroup of $C_{n}$, and is therefore flat on the quotient $\mathbb{P}^{n}$.

Note that just as for more general Coxeter groups, we can describe the $t$-independent conditions on sections of these sheaf categories in terms of residue conditions (at least generically). This does not quite follow from that case, as we are working in an extended affine Weyl group rather than an affine Weyl group, and of course the twisting makes things trickier.

For $\mathcal{H}_{-q-(n-1) t ; q ; t}^{(n)}\left(0, d s+d^{\prime} f\right), \mathcal{H}_{-q-(n-1) t ; q, t}^{(n)}\left(0, d s+d^{\prime} f\right)$, the local sections are just local sections of the untwisted Hecke algebra up to twisting on the left by some $\mathcal{O}_{\mathbb{P}^{n}}(l)$, and thus satisfy the usual residue conditions: the coefficients are all meromorphic sections of the same bundle $\mathcal{O}_{\mathbb{P}^{n}}(1)$, and for any two elements of the extended affine Weyl group related by a reflection, the sum of the corresponding coefficients must be holomorphic along the reflection hypersurface. (Moreover, the polar divisors of the coefficients must be sums of reflection hypersurfaces, with a given reflection hypersurface appearing only if it appears in a residue condition). For the spherical algebra, the condition is analogous, giving a residue condition for any pair of distinct translations which are conjugates by a reflection.

In our case, the twisting is simple enough that we can express the residue conditions explicitly even in the presence of twisting. For the even case, we find that for sufficiently general $u, v$,

$$
\begin{aligned}
\left(\prod_{1 \leq i \leq n} \vartheta\left(v \pm z_{i}\right)^{d^{\prime}} \Gamma_{q}\left(-d q / 2-u \pm z_{i},-d q / 2-(n-1) t-\eta^{\prime}+u \pm z_{i}\right)\right)^{-1} \\
\times \mathcal{H}_{\eta^{\prime} ; q, t}^{(n)}\left(0, d s+d^{\prime} f\right) \prod_{1 \leq i \leq n} \Gamma_{q}\left(-u \pm z_{i},-(n-1) t-\eta^{\prime}+u \pm z_{i}\right)
\end{aligned}
$$

consists of operators with elliptic coefficients and preserving holomorphy (away from divisors depending on $u$ and $v$ ). (This is essentially just performing an elementary transformation as considered below.) Thus the residue conditions on $\mathcal{H}_{\eta^{\prime} ; q, t}^{(n)}\left(0, d s+d^{\prime} f\right)$ may be obtained by gauging 
the untwisted residue conditions. We may then use Propositions 2.11 and 2.12 to simplify the result. We give the conditions for the spherical category for simplicity; for the DAHA, the correction is the same except for those reflections preserving the corresponding coset, when the correction factor is necessarily trivial.

We find that sections of $\mathcal{S}_{\eta^{\prime} ; q, t}^{(n)}\left(0, d s+d^{\prime} f\right)$ satisfy the following $t$-independent residue conditions (in addition to $C_{n}$-invariance and the corresponding bounds on poles). Write such a local section as $\sum_{\vec{k}} c_{\vec{k}}(\vec{z}) \prod_{i} T_{i}^{k_{i}}$, where each $k_{i}$ is congruent to $d / 2$ modulo $\mathbb{Z}$. First, for $m \neq k_{1}+k_{2}$,

$$
c_{m-k_{2}, m-k_{1}, k_{3}, \ldots, k_{n}}(\vec{z})+\left[\frac{\vartheta\left(u, q+(n-1) t+\eta^{\prime}+u+z_{1}+z_{2}+m q\right)}{\vartheta\left(u+z_{1}+z_{2}+m q, q+(n-1) t+\eta^{\prime}+u\right)}\right]^{2\left(m-k_{1}-k_{2}\right)} c_{k_{1}, k_{2}, k_{3}, \ldots, k_{n}}(\vec{z})
$$

is holomorphic along $z_{1}+z_{2}+m q=0$, where $u$ is arbitrary subject to $u \neq 0$ and $u \neq-q-$ $(n-1) t-\eta^{\prime}$ and otherwise has no effect on the condition, and similarly when $m \neq 2 k_{1}$,

$$
c_{m-k_{1}, k_{2}, \ldots, k_{n}}(\vec{z})+\left[\frac{\vartheta\left(u, q+(n-1) t+\eta^{\prime}+u+2 z_{1}+m q\right)}{\vartheta\left(u+2 z_{1}+m q, q+(n-1) t+\eta^{\prime}+u\right)}\right]^{m-2 k_{1}} c_{k_{1}, k_{2}, \ldots, k_{n}}(\vec{z})
$$

is holomorphic along $2 z_{1}+m q=0$. (The conditions along other reflection hyperplanes follow by $C_{n}$-invariance.) Moreover, for generic parameters, any operator satisfying these conditions and the appropriate $t$-dependent vanishing conditions will be a section of the given Hom sheaf. (To be precise, we must assume that $q$ is not torsion (or we are in a faithful Bruhat interval) and that $t$ is not a multiple of $q$; of course, by flatness, to check that a family of operators is a section of the corresponding family of categories $\mathcal{S}$, it suffices to verify that this holds generically.)

Similarly, for $\mathcal{S}_{x_{0} ; q, t}^{\prime(n)}\left(0, d s+d^{\prime} f\right)$, the correction factors in the residue conditions are

$$
\left[\frac{\vartheta\left(u, q+(n-1) t+x_{0}+u+z_{1}+z_{2}+m q\right)}{\vartheta\left(u+z_{1}+z_{2}+m q, q+(n-1) t+x_{0}+u\right)}\right]^{2\left(m-k_{1}-k_{2}\right)}
$$

and

$$
\left[\mathfrak{q}\left(z_{1}+m q / 2\right) \frac{\vartheta\left(u, q+(n-1) t+x_{0}+u+2 z_{1}+m q\right)}{\vartheta\left(u+2 z_{1}+m q, q+(n-1) t+x_{0}+u\right)}\right]^{m-2 k_{1}}
$$

where we recall that in characteristic not $2, \mathfrak{q} \in \mu_{2}(E[2])$ is the function taking 0 to 1 and nontrivial 2-torsion points to -1 . (Note that without the appearance of $\mathfrak{q}$ in the residue conditions, the subcategory $\mathcal{S}^{\prime}$ with objects $2 \mathbb{Z} s+\mathbb{Z} f$ would be a simple reparametrization of the corresponding subcategory of $\mathcal{S}$.)

By comparison with the univariate case (which we will discuss in more detail shortly), we are led to define generalizations ("blowups") of these algebras with even more parameters. Recall that local sections of the spherical algebras are difference operators. We let $T_{i}$ denote the operator that pulls back through $z_{i} \mapsto z_{i}+q$; note that in terms of our convention for how group elements act, $T_{i}$ is the same as the action of the translation $z_{i} \mapsto z_{i}-q$. We extend this to half-integer powers of $T_{i}$ by using the chosen $q / 2$. Every local section is then a left linear combination of monomials $T^{\vec{k}}:=\prod_{i} T_{i}^{k_{i}}$ in which all $k_{i}$ are half-integers in the same coset of $\mathbb{Z}$ (determined by the coefficient of $s$ in the degree of the operator in the category).

Definition 7.5. The sheaf category $\mathcal{S}_{\eta^{\prime}, x_{1}, \ldots, x_{m} ; q, t}^{(n)}$ is the sheaf category on $\mathbb{P}^{n} / \mathcal{E}^{m+3}$ with objects $\mathbb{Z}\left\langle s, f, e_{1}, \ldots, e_{m}\right\rangle$ defined by taking $\mathcal{S}_{\eta^{\prime}, x_{1}, \ldots, x_{m} ; q, t}^{(n)}\left(d_{1} s+d_{1}^{\prime} f-r_{11} e_{1}-\cdots-r_{1 m} e_{m}, d_{2} s+d_{2}^{\prime} f-r_{21} e_{1}-\right.$ $\left.\cdots-r_{2 m} e_{m}\right)$ to be the subsheaf of $\mathcal{S}_{\eta^{\prime} ; q, t}^{(n)}\left(d_{1} s+d_{1}^{\prime} f, d_{2} s+d_{2}^{\prime} f\right)$ consisting (locally) of operators $\mathcal{D}$ such that the left coefficient of $\prod_{1 \leq i \leq n} T_{i}^{k_{i}}$ vanishes on the divisors $z_{i}=x_{j}-\left(2 l-d_{2}+1\right) q / 2$ for $1 \leq i \leq n, 1 \leq j \leq m, k_{i}+\left(d_{2}-d_{1}\right) / 2+r_{1 j} \leq l<r_{2 j}$ and the divisors $z_{i}=-x_{j}+\left(2 l-d_{2}+1\right) q / 2$ for $1 \leq i \leq n, 1 \leq j \leq m,-k_{i}+\left(d_{2}-d_{1}\right) / 2+r_{1 j} \leq l<r_{2 j}$. Similarly, the sheaf category $\mathcal{S}_{x_{0}, x_{1}, \ldots, x_{m} ; q, t}^{\prime(n)}$ is the subsheaf category of $\mathcal{S}_{x_{0} ; q, t}^{(n)}$ satisfying the same vanishing conditions. 
Remark. Note that by symmetry, it would have sufficed to impose the first set of vanishing conditions.

Note that in this definition, we need to impose the vanishing conditions on the family as a whole; on individual fibers, the condition along individual divisors may be too strong (when the divisors are reflection hypersurfaces) or too weak (when the divisors are not distinct). As a result, it is a nontrivial question whether the family is flat, and even when it is flat, it is conceivable that the individual fibers might fail to inject in the category of meromorphic operators (which could in turn allow the fibers to acquire zero divisors).

Luckily, since we defined these using the spherical algebra of an elliptic DAHA, there is an obvious approach to studying these categories: construct them as spherical subquotients of a suitable subcategory of $\mathcal{H}_{\eta^{\prime} ; q, t}^{(n)}$ or $\mathcal{H}_{x_{0} ; q, t}^{\prime(n)}$. There are actually multiple choices one might make here, as one can view a divisor $z_{i}=x_{j}-k q / 2$ as a pullback from the coroot curve associated to either endpoint. Although it might seem natural to choose the endpoint that matches the parity of $k$, it will be easier for present purposes to consistently use $s_{0}$. Other choices may lead to more natural Hecke algebras, however; for instance the classical double affine Hecke algebra of type $C^{\vee} C_{n}$ corresponds to assigning two parameters to $s_{n}$ and two parameters to $s_{0}$.

Since we are keeping the parameters away from the finite Weyl group, the vanishing conditions should be appropriately $C_{n}$-equivariant, and thus the vanishing condition for the left coefficient of $w$ should depend only on $w C_{n}$ and be equivariant on the left. Each coset of $w C_{n}$ has a unique representative $\prod_{i} T_{i}^{k_{i}}$, and we readily verify that the vanishing conditions we imposed above transform well under $C_{n}$. We may thus define $\mathcal{H}_{\eta^{\prime}, x_{1}, \ldots, x_{m} ; q, t}^{(n)}$ by imposing the resulting vanishing conditions, and similarly for $\mathcal{H}_{x_{0}, x_{1}, \ldots, x_{m} ; q, t}^{\prime(n)}$.

We note that in addition to the functoriality on fibers implied by the fact that this is defined over the moduli stack, we also have functoriality with respect to translation by 2 -torsion.

Proposition 7.6. If $\tau$ is an fppf-local section of $\mathcal{E}[2]$, then there are isomorphisms

$$
\begin{aligned}
\mathcal{H}_{\eta^{\prime}, x_{1}, \ldots, x_{m} ; q, t}^{(n)} & \cong \mathcal{H}_{\eta^{\prime}, x_{1}+\tau, \ldots, x_{m}+\tau ; q, t}^{(n)}, \\
\mathcal{H}_{x_{0}, x_{1}, \ldots, x_{m} ; q, t}^{(n)} & \cong \mathcal{H}_{x_{0}+\tau, x_{1}+\tau, \ldots, x_{m}+\tau ; q, t}^{(n)} .
\end{aligned}
$$

Proof. Conjugating by the involution $\left(z_{1}, \ldots, z_{n}\right) \mapsto\left(z_{1}+\tau, \ldots, z_{n}+\tau\right)$ induces such an isomorphism on the generators for $m=0$ and acts as described on the additional vanishing conditions for $m>0$.

Remark 7.7. In fact, we could have defined these categories over the moduli stack of hyperelliptic curves of genus 1 , at the cost of making the action of $C_{n}$ slightly more complicated (with $s_{n}$ acting by $z_{n} \mapsto s\left(z_{n}\right)$, where $s$ is the hyperelliptic involution), in which case these isomorphisms follow by functoriality. This would have made a number of later formulas more complicated, as well as making it more difficult to discuss line bundles and $\Gamma_{q}$ symbols.

Remark 7.8. The above isomorphism involves translation by $\tau$ in every degree. If one instead only translates in the odd degrees, none of the parameters visible in the notation change, but $q / 2$ is replaced by $q / 2+\tau$. In particular, the algebra is indeed independent of the choice of $q / 2$.

We have the following useful "elementary transformation" symmetry. Here and below, we simplify things by observing that our sheaf categories satisfy a natural translation symmetry in which translation in the group of objects corresponds to translations of the parameters by multiples of $q / 2$, and thus it suffices to consider Hom bimodules starting at the 0 object.

Proposition 7.9. There are (locally on the base) natural isomorphisms

$$
\mathcal{H}_{x_{0}, x_{1}, x_{2}, \ldots, x_{m} ; q, t}^{(n)}\left(0, d s+d^{\prime} f-r_{1} e_{1}-\cdots-r_{m} e_{m}\right)
$$




$$
\begin{aligned}
\cong & \prod_{1 \leq i \leq n} \Gamma_{q}\left(\left(r_{1}+(1-d) / 2\right) q-x_{1} \pm z_{i}\right) \\
& \times \mathcal{H}_{x_{0}-x_{1},-x_{1}, x_{2}, \ldots, x_{m} ; q, t}^{(n)}\left(0, d s+\left(d^{\prime}-r_{1}\right) f-\left(d-r_{1}\right) e_{1}-r_{2} e_{2}-\cdots-r_{m} e_{m}\right) \\
& \times \prod_{1 \leq i \leq n} \Gamma_{q}\left(q / 2-x_{1} \pm z_{i}\right)^{-1}
\end{aligned}
$$

and

$$
\begin{aligned}
& \mathcal{H}_{\eta^{\prime}, x_{1}, x_{2}, \ldots, x_{m} ; q, t}^{(n)}\left(0, d s+d^{\prime} f-r_{1} e_{1}-\cdots-r_{m} e_{m}\right) \\
& \cong \prod_{1 \leq i \leq n} \Gamma_{q}\left(\left(r_{1}+(1-d) / 2\right) q-x_{1} \pm z_{i}\right) \\
& \times \mathcal{H}_{\eta^{\prime}-x_{1},-x_{1}, x_{2}, \ldots, x_{m} ; q, t}^{\prime(n)}\left(0, d s+\left(d+d^{\prime}-r_{1}\right) f-\left(d-r_{1}\right) e_{1}-r_{2} e_{2}-\cdots-r_{m} e_{m}\right) \\
& \times \prod_{1 \leq i \leq n} \Gamma_{q}\left(q / 2-x_{1} \pm z_{i}\right)^{-1} .
\end{aligned}
$$

Proof. It is easy to see that the corresponding categories of meromorphic operators are isomorphic (locally on the base), and the pseudo-conjugation by $\Gamma_{q}$ symbols respects the twisting data, so the image of the right-hand side in the meromorphic category corresponding to the left-hand side satisfies the same conditions on the reflection hypersurfaces. The conditions along the divisors corresponding to $x_{i}$ for $2 \leq i \leq m$ are clearly the same, and it is straightforward to check that the same holds for $i=1$.

Remark. Since the definition is also clearly invariant under permutations of $x_{1}, \ldots, x_{m}$, we may apply this symmetry in any $x_{i}$, and then by composition in any subset of the $x_{i}$. If the subset has odd size, then the isomorphism switches between $\mathcal{H}^{(n)}$ and $\mathcal{H}^{\prime(n)}$, while even subsets induce isomorphisms between extended DAHAs of the same parity. In particular, for each of the two families, there is an action of $W\left(D_{m}\right)$ on the parameter space that extends to an action on the family of sheaf categories.

If we can show that these sheaf categories have well-behaved Bruhat filtrations (i.e., in which the subquotients are obtained from the $m=0$ case by imposing the vanishing conditions), then the same will immediately hold for their spherical subquotients. Unfortunately, we cannot simply copy the arguments we used in the usual elliptic Hecke algebra case; although the upper bound on the Bruhat subquotients works the same way, the category structure means there is no longer a canonical way to associate a multiplication map to a reduced word.

There are some special cases which are easy, however. As before, we may restrict our attention to Hom bimodules starting from the 0 object.

The following is a trivial consequence of the definition.

Proposition 7.10. If $r_{m} \leq 0$, then

$$
\begin{aligned}
& \mathcal{H}_{\eta^{\prime}, x_{1}, \ldots, x_{m} ; q, t}^{(n)}\left(0, d s+d^{\prime} f-r_{1} e_{1}-\cdots-r_{m} e_{m}\right) \\
& \quad=\mathcal{H}_{\eta^{\prime}, x_{1}, \ldots, x_{m-1} ; q, t}^{(n)}\left(0, d s+d^{\prime} f-r_{1} e_{1}-\cdots-r_{m-1} e_{m-1}\right), \\
& \begin{aligned}
\mathcal{H}_{x_{0}, x_{1}, \ldots, x_{m} ; q, t}^{\prime(n)} & \left(0, d s+d^{\prime} f-r_{1} e_{1}-\cdots-r_{m} e_{m}\right) \\
& =\mathcal{H}_{x_{0}, x_{1}, \ldots, x_{m-1} ; q, t}^{\prime(n)}\left(0, d s+d^{\prime} f-r_{1} e_{1}-\cdots-r_{m-1} e_{m}\right) .
\end{aligned}
\end{aligned}
$$

Applying the elementary transformation symmetry in $x_{m}$ means that the case $r_{m} \geq d$ is also straightforward to deal with. There is one more case which is nice. 
Proposition 7.11. There is a system of parameters $\vec{T}$ and a twisting datum $\gamma$ such that each Hom bimodule

$$
\mathcal{H}_{\eta^{\prime}, x_{1}, \ldots, x_{m} ; q, t}^{(n)}\left(0, d\left(2 s+2 f-e_{1}-\cdots-e_{m}\right)\right)
$$

is equal to the corresponding Bruhat interval in $\mathcal{H}_{\tilde{C}_{n} ; \vec{T} ; \gamma}\left(\mathcal{E}^{n+m+3}\right)$, and similarly for

$$
\mathcal{H}_{x_{0}, x_{1}, \ldots, x_{m} ; q, t}^{(n)}\left(0, d\left(2 s+3 f-e_{1}-\cdots-e_{m}\right)\right) .
$$

Proof. We can rephrase the vanishing conditions for generic parameters as stating that

$$
\prod_{\substack{1 \leq i \leq n \\ 1 \leq j \leq m}} \Gamma_{q}\left(q / 2-x_{j} \pm z_{i}\right) \mathcal{D} \prod_{\substack{1 \leq i \leq n \\ 1 \leq j \leq m}} \Gamma_{q}\left(q / 2-x_{j} \pm z_{i}\right)^{-1}
$$

has holomorphic coefficients. The cocycle in Cartier divisors associated to this product of $\Gamma_{q}$ symbols is precisely the right form to come from a system of parameters (associated to the orbit of $\left.s_{0}\right)$.

It turns out that it suffices to understand these cases.

Theorem 7.12. For any vector $v=d s+d^{\prime} f-r_{1} e_{1}-\cdots-r_{m} e_{m}$, the bounds on the Bruhat subquotients of $\mathcal{H}_{\eta^{\prime}, x_{1}, \ldots, x_{m} ; q, t}^{(n)}(0, v)$ and $\mathcal{H}_{x_{0}, x_{1}, \ldots, x_{m} ; q, t}^{\prime(n)}(0, v)$ coming from the vanishing conditions are saturated. In particular, both sheaf categories are locally free, and the map from any Hom bimodule to the sheaf bimodule of meromorphic operators is injective on fibers.

Proof. We first use the elementary transformation symmetry to replace all the cases with $r_{i} \geq d$ with cases with $r_{i} \leq 0$ (possibly changing the parity), and thus with $r_{i}=0$. In particular, we observe that the cases $d \in\{0,1\}$ of the theorem reduce to the subcase $r_{1}=\cdots=r_{m}=0$, and thus to the original elliptic DAHA, where we certainly have saturation. For $d>1$, if some $r_{i}=0$, we can simply omit that parameter and thus reduce to a case with smaller $m$. We thus find that it suffices to prove saturation when $0<r_{1}, \ldots, r_{m}<d$.

We consider the even case $\mathcal{H}^{(n)}$, with the odd case $\mathcal{H}^{\prime(n)}$ being entirely analogous. Let $C_{m}:=2 s+2 f-e_{1}-\cdots-e_{m}$, and suppose by induction that we have saturation for $v-C_{m}$. It will then suffice to show that the image of the multiplication map

$$
\mathcal{H}_{\eta^{\prime}, x_{1}, \ldots, x_{m} ; q, t}^{(n)}\left(v-C_{m}, v\right) \otimes \mathcal{H}_{\eta^{\prime}, x_{1}, \ldots, x_{m} ; q, t}^{(n)}\left(0, v-C_{m}\right) \rightarrow \mathcal{H}_{\eta^{\prime}, x_{1}, \ldots, x_{m} ; q, t}^{(n)}(0, v)
$$

saturates the bound. Since the first factor is a Bruhat interval in an honest elliptic DAHA, it in particular has a global section 1 , giving an inclusion

$$
\mathcal{H}_{\eta^{\prime}, x_{1}, \ldots, x_{m} ; q, t}^{(n)}\left(0, v-C_{m}\right) \subset \mathcal{H}_{\eta^{\prime}, x_{1}, \ldots, x_{m} ; q, t}^{(n)}(0, v),
$$

identifying the former with the Bruhat interval of the latter corresponding to the dominant weight $(d / 2-1, d / 2-1, \ldots, d / 2-1)$. The bounds on the subquotients are clearly the same, and thus we have saturation for any $w$ in this interval.

For the rest of the module, we note that both sides are bimodules over the finite Hecke algebra, and since the $x_{i}$ parameters have no effect on this algebra, we may reduce to the case of a Bruhat interval $[\leq w]$ with $w$ a minimal representative of $C_{n} \backslash \tilde{C}_{n}$. Let $\lambda(w)$ be the corresponding dominant weight. We have already shown that the leading coefficient map is saturated when $\lambda(w)_{1} \leq d / 2-1$, so without loss of generality may assume $\lambda(w)_{1}=d / 2$. It then follows from the structure of minimal coset representatives that not only is $s_{0} w<w$, but $\lambda\left(s_{0} w\right)$ is obtained from $\lambda(w)$ by reducing some coefficient from $d / 2$ to $d / 2-1$. This changes the bound on the leading coefficient bundle by precisely the leading coefficient divisor of $s_{0}$ in the relevant DAHA, and thus gives saturation as required. 
Passing to the spherical subquotient gives us the following.

Corollary 7.13. For any vector $v=d s+d^{\prime} f-r_{1} e_{1}-\cdots-r_{m} e_{m}$, the bounds on the Bruhat subquotients of $\mathcal{S}_{\eta^{\prime}, x_{1}, \ldots, x_{m} ; q, t}^{(n)}(0, v)$ and $\mathcal{S}_{x_{0}, x_{1}, \ldots, x_{m} ; q, t}^{(n)}(0, v)$ coming from the vanishing conditions are saturated. In particular, both sheaf categories are locally free, and the map from any Hom bimodule to the sheaf bimodule of meromorphic difference operators is injective on fibers.

As in the general DAHA situation, we would like to understand the centers of the extended DAHAs and their spherical algebras. There is a technical issue, however, in that the notion of center is not quite well-defined for general categories. More precisely, one might be inclined to define the center of a category to be the endomorphism algebra of the identity functor, but this is too small in our cases. We could fix the "too small" problem by instead taking the center to be the category with objects the automorphisms of the original category and morphisms given by natural transformations, but this is too large to be useful. Luckily, there is a happy medium: we take a suitable action of $\mathbb{Z}^{m+2}$ on the category such that the induced action on objects is just the usual action by translation.

This action is mostly straightforward: since every Hom space of degree $2 s+2 f$ (or $2 s+3 f$ in the odd case, at least locally on the base) contains the operator 1, and similarly for any Hom space of degree $e_{i}$, any element of the sublattice $\left\langle 2 s+2 f, e_{1}, \ldots, e_{m}\right\rangle$ induces an isomorphism between corresponding categories. Translation by $f$ is slightly more subtle, but corresponds to twisting by the equivariant bundle $\pi^{*} \mathcal{O}_{\mathbb{P}^{n}}(1)$, so again induces an isomorphism of categories. The result is index 2 in $\mathbb{Z}^{m+2}$, which is good enough for many purposes, but since we would like to be able to identify the center of the extended DAHA with one of our spherical algebras, we need to fill out the lattice. This is not too difficult in the extended DAHA, which contains a unique "element" of the form ${ }^{w} \mathcal{L} w \omega$ with $w \in C_{n}$ and $w \omega$ a translation. This is invertible, and again induces appropriate isomorphisms of categories. (Note, however, that its square is not actually compatible with the lattice of automorphisms already described.)

Now, suppose $q$ is torsion, of order $l$. If we take the $l$ th powers of the above isomorphisms, then all of the categories that arise can be identified with the original category, and we thus obtain the desired family of automorphisms (which became consistent after taking $l$ th powers, as the error was translation by $q$ ). Moreover, the automorphism corresponding to $l s$ of the extended DAHA is $W$-invariant, and thus extends to an automorphism of the spherical algebra. We define the "center" of the extended DAHA or its spherical algebra to be the category with objects $l \mathbb{Z}^{m+2}$ arising from the above construction.

The following is straightforward.

Lemma 7.14. If $q=0$, then the categories $\mathcal{S}^{(n)}$ and $\mathcal{S}^{(n)}$ are naturally isomorphic to their centers.

More generally, let $\pi_{q}: E \rightarrow E^{\prime}$ be the $l$-isogeny with kernel generated by $q$.

Theorem 7.15. The centers of $\mathcal{H}_{\eta^{\prime}, x_{1}, \ldots, x_{m} ; q, t}^{(n)}$ and $\mathcal{S}_{\eta^{\prime}, x_{1}, \ldots, x_{m} ; q, t}^{(n)}$ are naturally isomorphic to the pullback of $\mathcal{S}_{\pi_{q}\left(\eta^{\prime}\right), \pi_{q}\left(x_{1}\right), \ldots, \pi_{q}\left(x_{m}\right) ; 0, \pi_{q}(t)}^{(n)}$ from $E^{\prime}$.

Proof. The argument of Theorem 6.18 together with the flatness results for the spherical algebra allows us to reduce to the parameter-free case (defined in the obvious way). We may as well localize the central section 1 of degree $2 s+2 f$, as we can then recover the center of the category from the induced filtration on the center of the localization. Any invariant section of $\pi^{*} \mathcal{O}_{\mathbb{P} n}(l)$ is clearly central, and we can locally choose an invertible such section to deal with the automorphisms of degree $l f$. We obtain a description of the resulting sheaf of categories with objects $\mathbb{Z} / 2 \mathbb{Z}$ as a sheaf algebra over the corresponding $\mathcal{O}_{X^{+}}$. The even part of the center is precisely the center of the corresponding DAHA, while the odd part of the center may be 
identified with a submodule of the form $Z\left(\mathcal{H}_{\tilde{C}_{n}, \gamma}(X)\right) \mathcal{Z}_{w_{l}} w_{l}$, where $w_{l}$ is given by $w_{l} \omega^{l}=(w \omega)^{l}$, where $w \omega$ is a translation with $w \in W$. Thus the center satisfies strong flatness and saturates the appropriate Bruhat filtration, so agrees with the spherical algebra.

To understand the significance of our construction, we will need to understand some special cases. The case $t=0$ is of particular interest, due to the following. Note that the tensor and symmetric power constructions on modules extend to sheaf bimodules, and thus carry over to analogous constructions for algebras and categories. (Of course, in the latter cases, one should take the symmetric subobject, not the quotient object.) The following is an immediate consequence of the fact that the corresponding $A_{n-1}$ Hecke algebra is just the usual twisted group algebra.

Proposition 7.16. One has the following isomorphisms (locally on the base):

$$
\begin{aligned}
\mathcal{S}_{\eta^{\prime}, x_{1}, \ldots, x_{m} ; q, 0}^{(n)} & \cong \operatorname{Sym}^{n}\left(\mathcal{S}_{\eta^{\prime}, x_{1}, \ldots, x_{m} ; q, 0}^{(1)}\right), \\
\mathcal{S}_{x_{0}, x_{1}, \ldots, x_{m} ; q, 0}^{(n)} & \cong \operatorname{Sym}^{n}\left(\mathcal{S}_{x_{0}, x_{1}, \ldots, x_{m} ; q, 0}^{(1)}\right) .
\end{aligned}
$$

Remark. Note that the tensor product of $n$ univariate difference operators is described as follows. The $i$ th operator acts on $k\left(\mathcal{E}^{n}\right)$ by pulling back the coefficients from the $i$ th factor and only translating the $i$ th coordinate. These actions commute, and thus we may compose them to obtain the tensor product difference operator on $k\left(\mathcal{E}^{n}\right)$. The tensor product of the algebras is then the image of the tensor product of sheaves under this operation, and the symmetric power consists of those operators in the tensor product that commute with $S_{n}$.

There is a similar description for $t=q$ coming from the following symmetry (essentially Corollary 5.32, combined with the fact that the conditions associated to $x_{1}, \ldots, x_{m}$ are unaffected).

Proposition 7.17. One has the following isomorphisms (locally on the base):

$$
\begin{aligned}
& \mathcal{S}_{\eta^{\prime}, x_{1}, \ldots, x_{m} ; q, q-t}^{(n)} \cong \prod_{1 \leq i<j \leq n} \Gamma_{p, q}\left(t \pm z_{i} \pm z_{j}\right) \mathcal{S}_{\eta^{\prime}, x_{1}, \ldots, x_{m} ; q, t}^{(n)} \prod_{1 \leq i<j \leq n} \Gamma_{p, q}\left(t \pm z_{i} \pm z_{j}\right)^{-1}, \\
& \mathcal{S}_{x_{0}, x_{1}, \ldots, x_{m} ; q, q-t}^{(n)} \cong \prod_{1 \leq i<j \leq n} \Gamma_{p, q}\left(t \pm z_{i} \pm z_{j}\right) \mathcal{S}_{x_{0}, x_{1}, \ldots, x_{m} ; q, t}^{(n)} \prod_{1 \leq i<j \leq n} \Gamma_{p, q}\left(t \pm z_{i} \pm z_{j}\right)^{-1} .
\end{aligned}
$$

We also note the following version of the adjoint symmetry. It will be convenient to express the adjoint in terms of a formal density; in particular, $\mathrm{d} T$ simply represents a formal $\tilde{C}_{n}$-invariant measure.

Proposition 7.18. The adjoint with respect to the formal density

$$
\prod_{1 \leq i<j \leq n} \frac{\Gamma_{q}\left(t \pm z_{i} \pm z_{j}\right)}{\Gamma_{q}\left( \pm z_{i} \pm z_{j}\right)} \prod_{1 \leq i \leq n} \frac{1}{\Gamma_{q}\left( \pm 2 z_{i}\right)} \mathrm{d} T
$$

induces (locally on the base) contravariant isomorphisms

$$
\begin{aligned}
& \mathcal{S}_{\eta^{\prime}, x_{1}, \ldots, x_{m} ; q, t}^{(n)} \cong \mathcal{S}_{-\eta^{\prime},-x_{1}, \ldots,-x_{m} ; q, t}^{(n)}, \\
& \mathcal{S}_{x_{0}, x_{1}, \ldots, x_{m} ; q, t}^{(n)} \cong \mathcal{S}_{-x_{0},-x_{1}, \ldots,-x_{m} ; q, t}^{\prime(n)}
\end{aligned}
$$

acting on objects as $v \mapsto-v$. 
Remark. We will refer to this formal adjoint as the "Selberg" adjoint, as the formal density consists of the interaction terms in the elliptic Selberg integral. More generally, composing the Selberg adjoint with a sequence of elementary transformations gives an adjunction involving densities of the form

$$
\prod_{1 \leq i \leq n} \frac{\prod_{1 \leq j \leq k} \Gamma_{q}\left(u_{j} \pm z_{i}\right)}{\Gamma_{q}\left( \pm 2 z_{i}\right)} \prod_{1 \leq i<j \leq n} \frac{\Gamma_{q}\left(t \pm z_{i} \pm z_{j}\right)}{\Gamma_{q}\left( \pm z_{i} \pm z_{j}\right)} \mathrm{d} T
$$

where the $u_{j}$ depend on the $x_{j}$ and the specific domain and codomain objects. Here we should think of the operators as mapping between two different inner product spaces, so that the two formal integrals are against (slightly) different densities.

We were somewhat vague in our descriptions of the subquotients of the Bruhat filtration above, as the specific divisors that are forced into a given coefficient are somewhat complicated to describe in general. For the most part, though, the important information about the subquotients is not how they are built up out of divisors, but simply which line bundle one ends up with in the end. (Recall that the subquotients are obtained from line bundles which are invariant under some parabolic subgroup $W_{I}$ by descending to $X / W_{I}$ then taking the direct image to $X / C_{n}$.)

Propositions 7.16 and 7.17 make this information relatively straightforward to determine. Any filtered isomorphism preserves the Bruhat subquotients, and thus the associated polarizations must be invariant under $t \mapsto q-t$ (modulo line bundles on the base, that is). Modulo line bundles on the base, the $t$-dependent contribution to the polarization is linear, and thus the $t \mapsto q-t$ symmetry forces it to be trivial. In other words, the subquotients are (up to isomorphism local on the base) independent of $t$ and thus by the first proposition are determined by the subquotients for $n=1$. More precisely, for $t=0$, the line bundle on $\mathcal{E}^{n}$ associated to a given dominant weight is the outer tensor product of the univariate subquotients associated to the parts of the weight; one then descends to the quotient by the stabilizer in $C_{n}$ of the weight.

Helpfully, it turns out that the univariate case has already been studied. Let $\Gamma \mathcal{S}^{(n)}, \Gamma \mathcal{S}^{\prime(n)}$ denote the associated "global section" categories; more precisely, these are sheaves of categories on $\mathcal{E}^{m+3}$ in which each Hom sheaf is the direct image of the corresponding Hom bimodule. Since we included twisting by $\mathcal{O}_{\mathbb{P}^{n}}(1)$ in the definition of the category, one can recover $\mathcal{S}^{(n)}$ and $\mathcal{S}^{\prime(n)}$ from their global section categories: for each Hom bimodule $M$ in the sheaf category, the corresponding graded module (relative to the Segre embedding of $\mathbb{P}^{n} \times \mathbb{P}^{n}$ ) can be extracted from the global section category.

In [26], two families of categories $\mathcal{S}_{\eta, \eta^{\prime}, x_{1}, \ldots, x_{m} ; q, p}$ and $\mathcal{S}_{\eta, x_{0}, x_{1}, \ldots, x_{m} ; q, p}^{\prime}$ were constructed on an analytic curve $\mathbb{C}^{*} /\langle p\rangle$. These have the same group of objects as our categories, and an interpretation of the local sections of the Hom sheaves as difference operators. Moreover, the categories for general $m$ are cut out from the categories for $m=0$ by suitable vanishing conditions, while the categories for $m=0$ are described via explicit generators given in terms of theta functions. Switching from multiplicative to additive notation and replacing $\theta$ by $\vartheta$ then extends this to arbitrary curves. (In fact, [26] gave such an extension by specifying an explicit gauging by products of Gamma functions that makes everything elliptic, and observing that the elliptic functions extend. But of course one could do the same gauging in terms of $\vartheta$ and $\Gamma_{q}$ symbols, so the resulting categories are the same.)

Although those operators are not quite $C_{1}$-symmetric in our sense, they are close: indeed, each operator formally takes functions invariant under $z \mapsto\left(1-d_{1}\right) q+\eta-z$ to functions invariant under $z \mapsto\left(1-d_{2}\right) q+\eta-z$. This, of course, is easy enough to fix: if we base change to have an element $\eta / 2$ (and recall that we already have an element $q / 2$ ), then we can compose on both sides by a suitable translation to make the operator honestly $C_{1}$-symmetric. 
Proposition 7.19. Locally on the base, the global section categories are isomorphic to the $C_{1}$-symmetric versions of the categories $\mathcal{S}, \mathcal{S}^{\prime}$ constructed in [26]. More precisely, if $v, w$ are arbitrary elements of the object group $\mathbb{Z}\left\langle s, f, e_{1}, \ldots, e_{m}\right\rangle$, then

$$
\begin{aligned}
& \Gamma \mathcal{S}_{\eta^{\prime}, x_{1}, \ldots, x_{m} ; q, t}^{(1)}(v, w) \cong \mathcal{S}_{2 c, 2 c+\eta^{\prime}, c+x_{1}, \ldots, c+x_{m} ; q ; \mathcal{E}}(v, w), \\
& \Gamma \mathcal{S}_{x_{0}, x_{1}, \ldots, x_{m} ; q, t}^{\prime(1)}(v, w) \cong \mathcal{S}_{2 c, c+x_{0}, c+x_{1}, \ldots, c+x_{m} ; q ; \mathcal{E}}^{\prime}(v, w),
\end{aligned}
$$

where $c$ is a free parameter.

Proof. For $m=0, \mathcal{S}_{2 c, 2 c+\eta^{\prime} ; q ; \mathcal{E}}$ and $\mathcal{S}_{2 c, c+x_{0} ; q ; \mathcal{E}}^{\prime}$ are generated in degrees $f, s, s+f$. The degree $f$ operators are clearly elements of the corresponding global section category, and the $C_{1}$-symmetry along with the fact that the only poles are $\left[X^{s_{1}}\right]$ implies the same for the degree $s$ and $s+f$ operators. Since the subcategory generated in this way saturates the Bruhat filtration, the categories are actually isomorphic.

For each of the four categories, every Hom sheaf for $m>0$ is contained in the appropriate Hom sheaf for $m=0$, and the image of a local section of $\mathcal{S}$ or $\mathcal{S}^{\prime}$ satisfies the correct vanishing conditions to be a local section of $\Gamma \mathcal{S}^{(1)}$ or $\Gamma \mathcal{S}^{\prime(1)}$. Moreover, the Bruhat filtration and the analogous filtration by order tells us that both Hom sheaves are direct images of vector bundles on $\mathbb{P}^{1}$ with the same Hilbert polynomials, and must therefore be identified by the isomorphism.

This leads to a particularly nice interpretation of our categories. If the rational surface $X_{m}$ is obtained from a Hirzebruch surface $X_{0}$ by blowing up $m$ points of a smooth anticanonical curve, then the line bundles on $X_{m}$ are parametrized by the group $\mathbb{Z}\left\langle s, f, e_{1}, \ldots, e_{m}\right\rangle$. This then gives rise to a category on this group of objects by taking the full subcategory of $\operatorname{coh}\left(X_{m}\right)$ in which the objects consist of one line bundle of each isomorphism class. We can, of course, do this over the entire moduli stack of such surfaces, which turns out (at least for $m>0$ ) to be isomorphic to $\mathcal{E}^{m+1}$. We then obtain a sheaf of categories on this base by taking the appropriate sheaf version of Hom between line bundles. It was shown in [26] that this sheaf of categories is precisely the specialization to $q=0$ of $\mathcal{S}$ or $\mathcal{S}^{\prime}$, depending on whether $X_{0}$ comes from a vector bundle of even or odd degree. (One caveat here is that the fibers in this category can be slightly different from the categories associated to individual surfaces; Hom spaces in the latter may jump in the presence of -2 -curves, while the global category is flat.)

The same, therefore, applies to our categories, and thus for all $n, \mathcal{S}_{\eta^{\prime}, x_{1}, \ldots, x_{m} ; 0,0}^{(n)}$ and $\mathcal{S}_{x_{0}, \ldots, x_{m} ; 0,0}^{(n)}$ can be interpreted as symmetric powers of rational surfaces. (To be precise, each fiber is equivalent to a subcategory of the subcategory of line bundles on such a symmetric power, which is full whenever the ratio of line bundles is acyclic.) The categories with $q=0$ and general $t$ are thus commutative deformations of such powers (some sort of compactified discrete elliptic Calogero-Moser spaces), while the categories with general $q, t$ are further noncommutative deformations.

We can also obtain analogous deformations for $\mathbb{P}^{2}$, though in that case only the global section category makes sense. If we restrict $\Gamma \mathcal{S}_{x_{0} ; q, t}^{(n)}$ to the objects in $\mathbb{Z}(s+f)$, then for $n=1, q=t=0$, we can identify consecutive Hom spaces in such a way as to obtain the polynomial algebra in three generators. (For $n=1, q \neq 0$, we instead get the three-generator Sklyanin algebra of $[1,3]$, see [26].) Thus for general $n, q=0$, we again obtain a family of commutative deformations of $\operatorname{Sym}^{n}\left(\mathbb{P}^{2}\right)$, and further noncommutative deformations for general parameters.

There are some caveats to the above discussion. One is that since we are including all line bundles in the construction, there is no canonical way to associate a projective variety for $q=0$ or a noncommutative analogue in general: in general, we would need to make an explicit choice of ample divisor, or make some other choice of what it means for a module over the category to be torsion (i.e., map to the 0 sheaf). For $n=1$, it was shown in [26] that any reasonable choice of ample divisor (in particular, any divisor which is ample on every $X_{m}$ ) gives rise to the 
same quotient category, and thus there is no difficulty. Unfortunately, the argument there relied heavily on showing that various product maps are surjective, and the analogous surjectivity fails for $n>1$ even for $q=t=0$. We therefore leave this as an open question.

There is also an issue here that, due to some difficulties in applying the Hecke algebra ideas to the $\mathbb{P}^{2}$ case, we cannot always prove flatness for general ample divisors. For any surface other than $\mathbb{P}^{2}$, this is not a significant issue, as there will always be a nonempty subcone of the ample cone for which everything does work as expected. For $\mathbb{P}^{2}$, or in general outside this subcone, we will at least be able to show that each Hom space is flat outside some finite (and most likely empty) set of bad pairs $(q, t)$.

We should also note that since the data in each case includes an explicit morphism to a Hirzebruch surface, that a priori the category might depend on this map (and, when $X_{0} \cong \mathbb{P}^{1} \times \mathbb{P}^{1}$, on the choice of ruling) and not just on the surface $X_{m}$. We will show in the following section that (just as for $n=1$ ) this is not an issue, but the argument is decidedly nontrivial.

Before proceeding to studying flatness for the global section category, we should note that there are also interpretations of $\mathcal{H}^{(1)}$ and $\mathcal{H}^{\prime(1)}$ in terms of the categories constructed in [26]. The point is that for $n=1$, we are taking a spherical algebra relative to a master Hecke algebra. Since this is the endomorphism algebra of a vector bundle on $\mathbb{P}^{1}$, we immediately find that the spherical algebra and the DAHA are Morita equivalent. (Note that even for generic parameters, this does not quite follow from Proposition 6.10, as this new Morita equivalence applies to the compactified versions of the algebras.) Making this Morita equivalence explicit gives the following.

Proposition 7.20. Let $v, w \in \mathbb{Z}\left\langle s, f, e_{1}, \ldots, e_{m}\right\rangle$. Then

$$
\Gamma \mathcal{H}_{\eta^{\prime}, x_{1}, \ldots, x_{m} ; q}^{(1)}(v, w) \cong\left(\begin{array}{cc}
\Gamma \mathcal{S}_{\eta^{\prime}, x_{1}, \ldots, x_{m} ; q}^{(1)}(v, w) & \Gamma \mathcal{S}_{\eta^{\prime}, x_{1}, \ldots, x_{m} ; q}^{(1)}(v-2 f, w) \\
\Gamma \mathcal{S}_{\eta^{\prime}, x_{1}, \ldots, x_{m} ; q}^{(1)}(v, w-2 f) & \Gamma \mathcal{S}_{\eta^{\prime}, x_{1}, \ldots, x_{m} ; q}^{(1)}(v-2 f, w-2 f)
\end{array}\right),
$$

and similarly for $\Gamma \mathcal{H}^{\prime(1)}$.

Remark. One can also apply this at the level of sheaf categories on $\mathbb{P}^{1}$. There one finds (per the analogous statement of [26]) that the spherical sheaf category is the sheaf "ZZ-algebra" associated to a noncommutative $\mathbb{P}^{1}$-bundle on $\mathbb{P}^{1}[38]$. There is, of course, no reason why we could not apply the Morita equivalence associated to $\mathcal{O}_{\mathbb{P}^{1}} \oplus \mathcal{O}_{\mathbb{P}^{1}}(-2)$ to any such noncommutative $\mathbb{P}^{1}$-bundle on $\mathbb{P}^{1}$ and thus obtain an associated DAHA (which will always be a degeneration of the elliptic DAHA).

In [26], it was shown that the algebras $\mathcal{S}$ satisfy a "Fourier transform" symmetry swapping $\eta$ and $\eta^{\prime}$ and swapping $s$ and $f$, which in turn induces a symmetry

$$
\Gamma \mathcal{S}_{\eta^{\prime}, x_{1}, \ldots, x_{m} ; q, 0}^{(1)} \cong \Gamma \mathcal{S}_{-\eta^{\prime}, x_{1}-\eta^{\prime} / 2, \ldots, x_{m}-\eta^{\prime} / 2 ; q, 0}^{(1)},
$$

again swapping $s$ and $f$. (We will show in the next section how to extend this to general $n$.) Since the description of $\Gamma \mathcal{H}^{(1)}$ in terms $\Gamma \mathcal{S}^{(1)}$ is not invariant under swapping $s$ and $f$, this symmetry does not actually extend to the DAHA itself. It turns out that, at least for $m=0$, this is a consequence of the compactification we performed. Each Hom space of degree $2 s+2 f$ contains an element 1 (in a Fourier-invariant way!); if we localize with respect to those elements, then the objects $v$ and $v+2 s+2 f$ of the category become isomorphic, and thus we may replace $v-2 f$ by $v+2 s$ in the above description. Using the translation symmetry, we can then subtract $2 s$ from $v$ and $v+2 s$ at the cost of changing the parameters slightly. But then swapping $s$ and $f$ recovers the above description of $\Gamma \mathcal{H}^{(1)}$. In other words, the localized DAHAs actually do satisfy a Fourier transformation symmetry (though it is not clear how to describe it in terms of explicit 
operators). It is likely that something similar holds in general (including $n>1$ ), but this will require a better understanding of the relevant Morita equivalences.

In the univariate setting, one can gain some insight from the results of [21] on the traditional $C^{\vee} C_{1}$ Hecke algebra. This suggests in general that replacing $-2 f$ above by $-2 f+e_{1}+\cdots+e_{k}$ would have the effect of moving the parameters $x_{1}, \ldots, x_{k}$ from $s_{0}$ to $s_{n}$. If so, then the Fourier transform would continue to extend to the noncompact elliptic DAHA in the presence of non- $t$ parameters, but would effectively swap the roles of the two roots vis-à-vis the parameters.

This degeneration also gives strong evidence that the full $\mathrm{SL}_{2}(\mathbb{Z})$ action will not extend to the elliptic DAHA (compact or not). Indeed, if one looks at the action of $\mathrm{SL}_{2}(\mathbb{Z})$ on the corresponding surfaces in the case that the spherical algebra is commutative, one finds that it relies on the fact that the anticanonical curve at infinity is singular. Each generator of $\mathrm{SL}_{2}(\mathbb{Z})$ blows up a singular point and then blows down a different component of the anticanonical curve, so that the anticanonical curve has the same shape and its complement has not changed, but the actual projective surface has. Blowing up a smooth point of the anticanonical curve in general does change the complement of the anticanonical curve, and thus we cannot expect this operation to survive to the elliptic level.

As with sheaves in general, when we take global sections in a family of sheaf categories, the fibers of the global sections can differ considerably from the global sections of the fibers. That is, there is a natural morphism from each fiber of the global section category to the global section category of the corresponding fiber, but this morphism can fail to be either injective or surjective. The failure of injectivity is particularly bad when we consider that the kernel of the map does not inherit an interpretation in terms of difference operators. In particular, if we have such a failure of injectivity, then we can no longer be confident that the fiber is a domain.

Each Hom sheaf in the global section category is the direct image of the corresponding Hom bimodule, and we can factor the direct image through one of the projections $\mathbb{P}^{n} \times \mathbb{P}^{n} \rightarrow \mathbb{P}^{n}$ to find that the Hom sheaf is the direct image of a vector bundle on $\mathbb{P}^{n}$. If every fiber of that vector bundle is acyclic, then Grauert's theorem tells us that taking the direct image actually does commute with passing to fibers. It turns out that this holds (modulo some genericity assumptions in some cases) for a sufficiently large class of degrees to allow us to prove in general that the map is always injective and that the global section category is flat.

There are two ways to show acyclicity. One is to show that every subquotient of the Bruhat filtration is acyclic; the other is to use the symmetric power description for $t=0$ to deduce acyclicity for $t=0$ and thus in a neighborhood of $t=0$ by semicontinuity. In either case, the bundle is either itself a symmetric power or is built up out of symmetric powers, and thus we need to understand when such a bundle is acyclic.

Given a sheaf $M$ on a scheme $X$, we may define a sheaf $\operatorname{Sym}^{n}(M)$ on the symmetric power $\operatorname{Sym}^{n}(X)$ by descending $M^{\bigotimes n}$ through the quotient by $S_{n}$.

Lemma 7.21. Let $X$ be a projective scheme over a field $k$, and let $M$ be an acyclic sheaf on $X$. Then $\operatorname{Sym}^{n}(M)$ is an acyclic sheaf on $\operatorname{Sym}^{n}(X)$ for all $n \geq 1$.

Proof. First, note that if $k$ has characteristic $p>n$ or 0 , then this is immediate, since $\operatorname{Sym}^{n}(M)$ is a direct summand of an acyclic sheaf, namely the direct image of the acyclic sheaf $M^{\bigotimes n}$.

In general, we proceed by induction on the pair $(n, \operatorname{dim} X)$ relative to the product partial order. Let $\mathcal{O}_{X}(1)$ be a very ample divisor on $X$, and note that $\operatorname{Sym}^{n}\left(\mathcal{O}_{X}(1)\right)$ is at least ample on $\operatorname{Sym}^{n}(X)$. (It can fail to be very ample!) In particular, there exists $l>0$ so that

$$
\operatorname{Sym}^{n}(M) \otimes \operatorname{Sym}^{n}\left(\mathcal{O}_{X}(1)\right)^{l} \cong \operatorname{Sym}^{n}(M(l))
$$

is acyclic. Choose a nonzero section of $\mathcal{O}_{X}(l)$, and use it to embed $M$ as a subsheaf of $M(l)$. This one-step filtration of $M(l)$ induces a symmetric power filtration $F_{\bullet}$ of $\operatorname{Sym}^{n}(M(l))$ such 
that $F_{m+1} / F_{m}$ is the direct image on $\operatorname{Sym}^{n}(X)$ of the sheaf $\operatorname{Sym}^{m}(M(l) / M) \otimes \operatorname{Sym}^{n-m}(M)$ on $\operatorname{Sym}^{m}(X) \times \operatorname{Sym}^{n-m}(X)$. By induction each subquotient $F_{m+1} / F_{m}$ for $m>0$ is acyclic, as each factor is either a symmetric power of lower degree or supported on a lower-dimensional projective scheme. Since $F_{1}=\operatorname{Sym}^{n}(M)$, it follows that $\operatorname{Sym}^{n}(M(l)) / \operatorname{Sym}^{n}(M)$ is acyclic, and thus that $H^{p}\left(\operatorname{Sym}^{n}(M)\right)=0$ for $p>1$.

For all $m \geq 0$, we have $H^{0}\left(\operatorname{Sym}^{n}(M(m))\right) \cong \operatorname{Sym}^{n}\left(H^{0}(M(m))\right)$, and thus $h^{0}\left(\operatorname{Sym}^{n}(M(m))\right)$ $=\left(\begin{array}{c}h^{0}(M(m))+n-1 \\ n\end{array}\right)$. Applying this to $m \gg 0$ lets us compute the Hilbert polynomial of $\operatorname{Sym}^{n}(M)$, and then setting $m=0$ gives

$$
\chi\left(\operatorname{Sym}^{n}(M)\right)=\left(\begin{array}{c}
\chi(M)+n-1 \\
n
\end{array}\right)=\left(\begin{array}{c}
h^{0}(M)+n-1 \\
n
\end{array}\right)=h^{0}\left(\operatorname{Sym}^{n}(M)\right) .
$$

Since we have already shown that the higher cohomology spaces vanish, this implies that $h^{1}$ also vanishes, and the claim follows.

By considering subquotients for the Bruhat filtration, we obtain the following.

Lemma 7.22. Suppose $d^{\prime} \geq d-1$. Then every fiber of $\mathcal{S}_{\eta^{\prime} ; q, t}^{(n)}\left(0, d s+d^{\prime} f\right)$ is acyclic for the morphism to parameter space.

Lemma 7.23. Suppose $2 d^{\prime} / 3 \geq d-1$. Then every fiber of $\mathcal{S}_{x_{0} ; q, t}^{(n)}\left(0, d s+d^{\prime} f\right)$ is acyclic for the morphism to parameter space.

Lemma 7.24. Suppose $d^{\prime} \geq \max \left(d, d / 2+r_{1}\right)$ and $0 \leq r_{1} \leq d$. Then every fiber of $\mathcal{S}_{\eta^{\prime}, x_{1} ; q, t}^{(n)}(0, d s+$ $\left.d^{\prime} f-r_{1} e_{1}\right)$ is acyclic for the morphism to parameter space.

Proof. The first two lemmas are straightforward. For the third, note that if $r_{1} \leq d / 2$, then imposing the vanishing conditions subtracts $r_{1}$ from the degree of the top subquotient, $r_{1}-1$ from the next, etc., until we reach 0 , and in each case there is sufficient degree to do this without becoming negative (or 0 , apart from the subquotient supported on $\mathbb{P}^{1}$ ). For $r_{1}>d / 2$, we apply the elementary transformation symmetry to reduce to a $r_{1} \leq d / 2$ case with the opposite parity. Each case gives a convex cone in which we are guaranteed acyclicity, and combining the cones gives the desired result.

Proposition 7.25. Suppose $v=d s+d^{\prime} f-r_{1} e_{1}-\cdots-r_{m} e_{m}$ satisfies the inequalities $d^{\prime} \geq$ $\max \left(d, d / 2+r_{1}\right), d \geq r_{1}+r_{2}$ and $r_{1} \geq r_{2} \geq \cdots \geq r_{m} \geq 0$, and either $v=0$ or $2 d+2 d^{\prime}-r_{1}-r_{2}-$ $\cdots-r_{m}>0$. Then every fiber of $\mathcal{S}_{\eta^{\prime}, x_{1}, \ldots, x_{m} ; q, t}^{(n)}(0, v)$ is acyclic for the morphism to parameter space.

Proof. Every subquotient is the direct image under a finite morphism of an outer tensor product of symmetric powers of subquotients of the $n=1$ case. The constraints on $v$ ensure that every univariate subquotient is acyclic: either (the direct image of) a line bundle of positive degree on $\mathcal{E}$ or a line bundle of nonnegative degree on $\mathbb{P}^{1}$. It follows that every Bruhat subquotient for general $n$ is acyclic, and thus the same holds for the full Hom space.

To check the univariate assertion, note that for $d=0$, the vector bundle is simply $\mathcal{O}_{\mathbb{P}^{1}}\left(d^{\prime}\right)$, so there is no problem. For $d>0$, the leading subquotient in the filtration comes from a line bundle on $\mathcal{E}$ of degree $2 d+2 d^{\prime}-r_{1}-\cdots-r_{m}$, so is positive, and the corresponding subsheaf is the same as the Hom sheaf obtained by subtracting $2 s+2 f-e_{1}-\cdots-e_{m}$ from $v$ (unless $d=1$, when the subsheaf is trivial and there is nothing further to discuss). If $m>1$ (the $m=1$ case already having been dealt with), then this subtraction preserves all of the inequalities except possibly $r_{m} \geq 0$ and $2 d+2 d^{\prime}-r_{1}-\cdots-r_{m}>0$. The first inequality could only be violated if we had $r_{m}=0$, in which case we might as well have omitted that parameter. For the other inequality, we are adding $m-8$ to the left-hand side, so there is no problem if $m \geq 8$. But if $m<8$, then the inequality is implied by the other inequalities. 
Note that this proposition is already stronger than it seems, as we can always arrange to have the inequalities $d \geq r_{1}+r_{2}, r_{1} \geq r_{2} \geq \cdots \geq r_{m} \geq 0$ by applying a suitable combination of elementary transformations and setting negative $r_{i}$ to 0 . Indeed, with the exception of the final inequality, this is just stating that the vector $v$ is in the fundamental chamber for the corresponding action of $W\left(D_{m}\right)$. In particular, for any vector $v$, we can use this proposition to find an explicit $d^{\prime}$ such that $v+d^{\prime} f$ satisfies acyclicity. (The existence of such a $d^{\prime}$ was of course already guaranteed by Serre vanishing.) If $r_{1} \leq d / 2$, then this bound is pretty close to tight (based on what we know about the $n=1$ case), but for $r_{1} \geq d / 2$, the following result suggests that there is considerable room for improvement.

Proposition 7.26. Suppose $v=d s+d^{\prime} f-r_{1} e_{1}-\cdots-r_{m} e_{m}$ satisfies the inequalities $d^{\prime} \geq$ $d \geq r_{1}+r_{2} ; r_{1} \geq r_{2} \geq \cdots \geq r_{m} \geq 0$; and either $v=0$ or $2 d+2 d^{\prime}-r_{1}-r_{2}-\cdots-r_{m}>0$. Then there is a codimension $\geq 2$ subscheme of parameter space, not meeting the subschemes $t=0$ or $t=q$, such that every fiber of $\mathcal{S}_{\eta^{\prime}, x_{1}, \ldots, x_{m} ; q, t}^{(n)}(0, v)$ on the complement is acyclic for the morphism to parameter space.

Proof. If $n=1$, then these inequalities are enough to guarantee acyclicity, per [26]. It follows that any fiber with $t=0$ satisfies acyclicity, and thus the open subscheme on which acyclicity holds contains the divisor $t=0$. By the $t \mapsto q-t$ symmetry, the acyclic locus also contains the divisor $t=q$. This pair of divisors is relatively ample over $\mathcal{M}_{1,1}$ for the $\mathcal{E}^{2}$ parametrizing $q$ and $t$, and thus their complement contains no closed subscheme of codimension $\leq 1$.

We of course conjecture that the codimension $\geq 2$ subscheme is always empty.

Corollary 7.27. Suppose $v=d s+d^{\prime} f-r_{1} e_{1}-\cdots-r_{m} e_{m}$ satisfies the inequalities $d^{\prime} \geq d \geq r_{1}+r_{2}$ and $r_{1} \geq r_{2} \geq \cdots \geq r_{m} \geq 0$. Then there is a codimension $\geq 2$ subscheme of parameter space (empty if $d^{\prime} \geq d / 2+r_{1}$ and never meeting $t=0$ or $t=q$ ) on the complement of which $\Gamma \mathcal{S}_{\eta^{\prime}, x_{1}, \ldots, x_{m} ; q, t}^{(n)}(0, v)$ is flat and the map to meromorphic difference operators is injective on fibers.

Proof. If $v=0$ or $2 d+2 d^{\prime}-r_{1}-\cdots-r_{m}>0$, then this follows from acyclicity, so suppose $2 d+2 d^{\prime}-r_{1}-\cdots-r_{m} \leq 0$. This is the degree of the leading subquotient of the univariate filtration; if it is negative, then this leading subquotient never has a global section, while if it is 0 , the line bundle depends nontrivially on the parameters, and thus generically does not have a global section. Either way, the direct image of a nontrivial symmetric power of the leading univariate subquotient will always be 0 , and the same holds for an outer tensor product with such a power.

Consider a Bruhat order ideal (i.e., an order ideal in the poset of dominant weights) contained in the interval $[\leq(d / 2, \ldots, d / 2)]$. If this order ideal contains a dominant weight with $\lambda_{1}=d / 2$, then there is such a weight which is a maximal element of the order ideal. Since the subquotient corresponding to that maximal element has no direct image, removing it has no effect on the direct image. We thus find that the direct image of the interval $[\leq(d / 2, \ldots, d / 2)]$ is the same as the direct image of the interval $[\leq(d / 2-1, \ldots, d / 2-1)]$, and thus we reduce to $v-(2 s+$ $\left.2 f-e_{1}-\cdots-e_{m}\right)$ as before.

Remark. That the bad subscheme has codimension $\geq 2$ follows from general considerations; a failure of injectivity in codimension 1 comes from a local family of operators vanishing on a hypersurface, and we can always locally divide such a family by a function cutting out the hypersurface. Thus the true content is that the locus does not meet $t=0$ or $t=q$, and further has codimension $\geq 2$ image in the surface parametrizing $q$ and $t$. In particular, we have injectivity over the local ring at any point with $t=0$ or $t=q$, including the point $q=t=0$ corresponding to the undeformed case. 
For $0 \leq m \leq 7$ (or for $m=-1$, i.e., $\mathbb{P}^{2}$ ), the corresponding commutative surface is a (possibly singular) del Pezzo surface with a choice of smooth anticanonical curve and a sequence of blowdowns to a Hirzebruch surface. The anticanonical embedding of this surface is given by the graded algebra

$$
\bigoplus_{d \geq 0} \Gamma \mathcal{S}_{\eta^{\prime}, x_{1}, \ldots, x_{m} ; q, t}^{(1)}\left(0, d\left(2 s+2 f-e_{1}-\cdots-e_{m}\right)\right)
$$

we can interpret this as a graded algebra by using the fact that 1 is in

$$
\Gamma \mathcal{S}_{\eta^{\prime}, x_{1}, \ldots, x_{m} ; q, t}^{(1)}\left(d\left(2 s+2 f-e_{1}-\cdots-e_{m}\right),(d+1)\left(2 s+2 f-e_{1}-\cdots-e_{m}\right)\right)
$$

for any $d$. This is the Rees algebra of the natural filtration on the spherical algebra

$$
\bigcup_{d \geq 0} \Gamma \mathcal{S}_{\eta^{\prime}, x_{1}, \ldots, x_{m} ; q, t}^{(1)}\left(0, d\left(2 s+2 f-e_{1}-\cdots-e_{m}\right)\right)
$$

the coordinate ring of the complement of the chosen smooth anticanonical curve. Taking the multivariate versions thus gives deformations of the (anticanonically embedded) symmetric powers of $X_{m}$ and $X_{m} \backslash E$, and, apart from possible codimension $\geq 2$ exceptions for $\mathbb{P}^{2}$, these deformations are always flat, and every fiber is a domain.

If $X_{m}$ has -2-curves or $m>7$, then the anticanonical divisor is no longer ample, and thus one can no longer expect to obtain a deformation of $\operatorname{Sym}^{n}\left(X_{m}\right)$ as a graded algebra, or of $\operatorname{Sym}^{n}\left(X_{m} \backslash E\right)$ as a filtered algebra. This is why we generalized the spherical algebra construction to the above categories: one needs to work with some non-(pluri)anticanonical divisor, and it is then easier to include all divisors.

There is an interesting phenomenon that arises for the spherical algebra in the $m=8$ case. The global section algebra in this case is trivial (consisting only of the global section 1), but this merely reflects the fact that the generic fiber has no nontrivial global sections. The univariate subquotients in this case are all multiples of $x_{1}+\cdots+x_{8}-2 \eta^{\prime}$, and thus if this value is $r$-torsion, then any subquotient of weight a multiple of $r$ will be trivial. (In terms of surfaces, this corresponds to the case that $X_{8}$ is an elliptic surface, in which one fiber consists of $r$ copies of the chosen anticanonical curve.) As a result, the dimension of global sections of such a fiber in a given Bruhat interval can in principle be as large as the number of such weights contained in the interval, or (a priori) as small as 1.

It turns out that at least for $r=1$ (i.e., when the elliptic surface has a section), this upper bound is attained (i.e., the dimension of global sections in a Bruhat interval is equal to the size of the Bruhat interval), and furthermore those global sections satisfy a surprising property. Note that it suffices to find $n+1$ global sections of degree $2 s+2 f-e_{1}-\cdots-e_{8}$, as we can then obtain global sections with arbitrary dominant weight by taking products.

Theorem 7.28. On any fiber such that $2 \eta^{\prime}=x_{1}+\cdots+x_{8}$, the space of global sections of $\mathcal{S}_{\eta^{\prime}, x_{1}, \ldots, x_{8} ; q, t}^{(n)}\left(0,2 s+2 f-e_{1}-\cdots-e_{8}\right)$ is $n+1$-dimensional, and any two global sections commute.

Proof. Certainly, $n+1$ is an upper bound on the number of global sections, since there are $n+1$ subquotients, each of which has a unique global section. The given Hom bimodule is contained in the Hom bimodule $\mathcal{S}_{\eta^{\prime}, x_{1}, \ldots, x_{7} ; q, t}^{(n)}\left(0,2 s+2 f-e_{1}-\cdots-e_{7}\right)$, and the latter Hom bimodule satisfies acyclicity. Since the $m=7$ bimodule has 2 global sections when $n=1$, it has $n+1=\left(\begin{array}{c}2+n-1 \\ n\end{array}\right)$ global sections when $t=0$ and thus (by flatness) in general. We thus need to show that those global sections are actually global sections of the subsheaf we want.

Let $\mathcal{D}$ be such a global section. This is determined by the left coefficients $c_{m}$ of $\prod_{1 \leq i \leq m} T_{i}^{-1}$ for $0 \leq m \leq n+1$, where $c_{m}$ is $S_{m} \times C_{n-m}$-invariant. Each $c_{m}$ is a section of a line bundle $\mathcal{L}_{m}\left(D_{m}\right)$, where $\mathcal{L}_{m}$ comes from the equivariant gerbe and $D_{m}$ comes from the allowed 
poles and forced zeros. The allowed poles are somewhat complicated, since we are not assuming that $c_{m}$ is a leading coefficient, but the forced zeros are the same as they would have been if it were a leading coefficient. The symmetric power property then tells us that when $t=0$, the forced zeros associated to $t$ must all cancel allowed poles, and any allowed pole associated to a root of type $D_{n}$ must be cancelled in this way.

The remaining zeros and poles can be deduced from the univariate case, and we thus find that $c_{m}$ is a multiple of

$$
\begin{aligned}
& \prod_{1 \leq i<j \leq m} \frac{\vartheta\left(t-z_{i}-z_{j}, q+t-z_{i}-z_{j}\right)}{\vartheta\left(-z_{i}-z_{j}, q-z_{i}-z_{j}\right)} \prod_{\substack{1 \leq i \leq m \\
m<j \leq n}} \frac{\vartheta\left(t-z_{i} \pm z_{j}\right)}{\vartheta\left(-z_{i} \pm z_{j}\right)} \\
& \quad \times \prod_{1 \leq i \leq m} \frac{\prod_{1 \leq j \leq 7} \vartheta\left(q / 2+x_{j}-z_{i}\right)}{\vartheta\left(-2 z_{i}, q-2 z_{i}\right)} \prod_{m<j \leq n} \frac{1}{\vartheta\left(-q-2 z_{j}, q-2 z_{j}\right)},
\end{aligned}
$$

in the sense that the ratio is a holomorphic section of the line bundle with polarization

$$
\sum_{1 \leq i \leq m}\left(z_{i}^{2} / 2-\left(q / 2+x_{8}\right) z_{i}\right)+\sum_{m<j \leq n} 4 z_{i}^{2}
$$

modulo line bundles on the base. (The only difference between this and the leading coefficient of the corresponding Bruhat interval are the factors $\vartheta\left(-q-2 z_{i}, q-2 z_{i}\right)$ for $m<j \leq n$.) This line bundle has degree 1 in each $z_{i}$ for $1 \leq i \leq m$, so every holomorphic section has the same dependence on those variables, which we can read off from the polarization.

We thus conclude that $c_{m} / \prod_{1 \leq i \leq m} \vartheta\left(q / 2+x_{8}-z_{i}\right)$ is independent of $z_{1}$ through $z_{m}$, so is still holomorphic. As a result, we find that every global section of the $m=7$ bimodule is also a global section for $m=8$; more precisely, the holomorphy gives it generically, but the condition is closed, so it holds in general.

It remains to show commutativity. We first note as a sanity check that the $n+1$ leading term operators

$$
\prod_{1 \leq i<j \leq m} \frac{\vartheta\left(t-z_{i}-z_{j}, q+t-z_{i}-z_{j}\right)}{\vartheta\left(-z_{i}-z_{j}, q-z_{i}-z_{j}\right)} \prod_{\substack{1 \leq i \leq m \\ m<j \leq n}} \frac{\vartheta\left(t-z_{i} \pm z_{j}\right)}{\vartheta\left(-z_{i} \pm z_{j}\right)} \prod_{1 \leq i \leq m} \frac{\prod_{1 \leq j \leq 8} \vartheta\left(q / 2+x_{j}-z_{i}\right)}{\vartheta\left(-2 z_{i}, q-2 z_{i}\right)} T_{i}^{-1}
$$

commute. It follows that on any fiber with $x_{1}+\cdots+x_{8}=2 \eta^{\prime}$, the global section algebra of the spherical algebra

$$
\bigcup_{d \geq 0} \mathcal{S}_{\eta^{\prime}, x_{1}, \ldots, x_{8} ; q, t}^{(n)}\left(0, d\left(2 s+2 f-e_{1}-\cdots-e_{8}\right)\right)
$$

has abelian associated graded algebra; the above leading term operators give one element for each fundamental weight, so generate the associated graded algebra.

This global section algebra has a particularly nice symmetry: it is preserved by the formal adjoint with respect to the density

$$
\prod_{1 \leq i \leq n} \frac{\prod_{1 \leq j \leq 8} \Gamma_{q}\left(q / 2+x_{j} \pm z_{i}\right)}{\Gamma_{q}\left( \pm 2 z_{i}\right)} \prod_{1 \leq i<j \leq n} \frac{\Gamma_{q}\left(t \pm z_{i} \pm z_{j}\right)}{\Gamma_{q}\left( \pm z_{i} \pm z_{j}\right)} \mathrm{d} T .
$$

Indeed, this is the composition of the Selberg adjoint and all 8 elementary transformations, with the total effect on the parameters being $\eta^{\prime} \mapsto x_{1}+\cdots+x_{8}-\eta^{\prime}=\eta^{\prime}$. Note that although such isomorphisms are usually only defined up to a unit, we can eliminate that freedom by insisting that the adjoint of 1 be 1 . This is triangular with respect to Bruhat order, and is trivial 
on the associated graded algebra, since it fixes the generators and the associated graded algebra is abelian. Since a triangular involution which is 1 on the diagonal is 1 , we find that this formal adjoint acts trivially on the entire global section algebra. Since an algebra consisting entirely of self-adjoint operators is abelian, we conclude that the generators commute as required.

Since the $n+1$ operators are filtered by Bruhat order, it is natural from an integrable systems perspective to designate the first nontrivial operator (with leading term $\propto T_{1}^{-1}$ ) as the Hamiltonian. This has leading coefficient

$$
\frac{\prod_{1 \leq j \leq 8} \vartheta\left(q / 2+x_{j}-z_{1}\right)}{\vartheta\left(-2 z_{1}, q-2 z_{1}\right)} \frac{\prod_{2 \leq j \leq n} \vartheta\left(t-z_{1} \pm z_{j}\right)}{\prod_{2 \leq j \leq n} \vartheta\left(-z_{1} \pm z_{j}\right)},
$$

which turns out to be a mild reparametrization of the leading coefficient of the Hamiltonian proposed by van Diejen in [6] (see also [7, equations (3.12)-(3.14)], with the caveat that one must gauge the operator), and later shown to be integrable in [16]. In fact, one can verify (we omit the details) that van Diejen's operator satisfies the appropriate residue and vanishing conditions to be a global section of $\mathcal{S}_{\eta^{\prime}, x_{1}, \ldots, x_{8} ; q, t}^{(n)}\left(0,2 s+2 f-e_{1}-\cdots-e_{8}\right)$, and thus we have given a new proof that van Diejen's Hamiltonian is integrable.

Remark. Since our Hecke algebra methods gave a new proof of the existence of the commuting operators which were constructed in [16], it is natural to wonder whether there might be applications in the other direction; that is, using their $R$-matrix based approach to construct global sections of other Hom sheaves in our spherical DAHA categories. Such a construction might make it possible to prove flatness in general without having to exclude a codimension $\geq 2$ subscheme; if a given Hom bimodule generically has $N$ global sections, then to prove flatness and injectivity in a neighborhood of a given fiber, it suffices to construct $N$ local sections on a neighborhood of the fiber such that the restrictions to the fiber are linearly independent.

The connection to elliptic surfaces suggests a possible generalization of this integrable system. If $x_{1}+\cdots+x_{8}-2 \eta^{\prime}$, instead of being 0 , is a torsion point of order $r$, then we again find that there are many trivial Bruhat subquotients, and thus it becomes nontrivial to determine how many global sections the spherical algebra has. We cannot answer this in general, but we can, at least, show that the $r$-torsion condition forces there to be some nontrivial global sections.

Proposition 7.29. Let $E$ be an elliptic curve and $\eta^{\prime}, x_{1}, \ldots, x_{8}, q, t$ be points of $E$ such that $x_{1}+\cdots+x_{8}-2 \eta^{\prime}$ is a torsion point of order $r$. Then the corresponding fiber of the spherical algebra $\bigcup_{d} \mathcal{S}_{\eta^{\prime}, x_{1}, \ldots, x_{8} ; q, t}^{(n)}\left(0, d\left(2 s+2 f-e_{1}-\cdots-e_{8}\right)\right)$ has a global section of dominant weight $(r, 0, \ldots, 0)$ with nonzero leading term.

Proof. Indeed, every subquotient in the Bruhat filtration for the order ideal $[<(r, 0, \ldots, 0)]$ is acyclic: the bottom subquotient is $\mathcal{O}_{P^{n}}$, while the remaining subquotients are nontrivial elements of $E^{n}[r]$.

It is then natural to conjecture that the resulting Hamiltonian is integrable, or more precisely the following.

Conjecture 7.30. Under the same hypotheses, the fiber of

$$
\mathcal{S}_{\eta^{\prime}, x_{1}, \ldots, x_{8} ; q, t}^{(n)}\left(0, r\left(2 s+2 f-e_{1}-\cdots-e_{8}\right)\right)
$$

has $n+1$ global sections, all of which commute. 
Both parts of the proof for $r=1$ fail here: the $m=7$ surface has too many global sections, and the adjoint no longer gives an element of the same spherical algebra. There is some experimental evidence for this conjecture, however: for $n=r=2$, the analogous statement for a suitable degeneration to a nodal curve holds by a computer calculation. (We will briefly discuss how to construct such degenerations at the end of the next section.) This statement is, of course, trivial for $n=1$ (given the proposition), but it is worth noting there that the global sections of $\mathcal{S}_{\eta^{\prime}, x_{1}, \ldots, x_{8} ; 0,0}^{(1)}\left(0, r\left(2 s+2 f-e_{1}-\cdots-e_{8}\right)\right)$ are just the pullback of the global sections of $\mathcal{O}_{\mathbb{P}^{1}}(1)$ from the base of the elliptic fibration.

\section{The (spherical) $C^{\vee} C_{n}$ Fourier transform}

Our objective in the present section is to prove the following result.

Theorem 8.1. There is, locally on the base, an isomorphism

$$
\Gamma \mathcal{S}_{2 c, x_{1}, \ldots, x_{m} ; q, t}^{(n)} \cong \Gamma \mathcal{S}_{-2 c, x_{1}-c, \ldots, x_{m}-c ; q, t}^{(n)}
$$

acting on objects as $d s+d^{\prime} f-r_{1} e_{1}-\cdots-r_{m} e_{m} \mapsto d^{\prime} s+d f-r_{1} e_{1}-\cdots-r_{m} e_{m}$ and triangular with respect to the Bruhat filtration. Moreover, this isomorphism commutes (up to local units) with the Selberg adjoint.

We refer to this isomorphism as the "Fourier transform": in particular, note that it takes multiplication operators (of degree $d^{\prime} f$ ) to difference operators (of degree $d^{\prime} s$ ) and (at least on the parameters) is an involution. (In addition, though we will not be using this fact, the Fourier transform can be represented in the analytic setting by a formal integral operator [27].)

Before constructing the Fourier transform, we give some consequences. The simplest is that we can conjugate the symmetry by an elementary transformation.

Corollary 8.2. There is, locally on the base, an isomorphism

$$
\Gamma \mathcal{S}_{x_{1}+2 c, x_{1}, x_{2}, \ldots, x_{m} ; q, t}^{(n)} \cong \Gamma \mathcal{S}_{x_{1}-c, x_{1}+c, x_{2}-c, \ldots, x_{m}-c ; q, t}^{(n)}
$$

acting on objects as $d s+d^{\prime} f-r_{1} e_{1}-r_{2} e_{2}-\cdots-r_{m} e_{m} \mapsto\left(d^{\prime}-r_{1}\right) s+d^{\prime} f-\left(d^{\prime}-d\right) e_{1}-r_{2} e_{2}-$ $\cdots-r_{m} e_{m}$.

This also tells us that the deformations of $\mathbb{P}^{2}$ we constructed are independent of $x_{0}$ (as one would expect).

Corollary 8.3. The restriction to $\mathbb{Z}(s+f)$ of $\Gamma \mathcal{S}_{x_{0} ; q, t}^{(n)}$ is (fppf locally) independent of $x_{0}$.

Proof. The previous corollary gives (locally) an isomorphism $\Gamma \mathcal{S}_{x_{0}, x_{0}-2 c ; q, t}^{(n)} \cong \Gamma \mathcal{S}_{x_{0}-3 c, x_{0}-c ; q, t}^{(n)}$. The action on objects takes $d(s+f)$ to $d(s+f)$, so this local isomorphism induces a local isomorphism $\left.\left.\Gamma \mathcal{S}_{x_{0} ; q, t}^{\prime(n)}\right|_{\mathbb{Z}(s+f)} \cong \Gamma \mathcal{S}_{x_{0}-3 c ; q, t}^{\prime(n)}\right|_{\mathbb{Z}(s+f)}$ for any $x_{0}$ and $c$. It follows that any two geometric fibers with the same values of $q, t$ are isomorphic.

Remark 8.4. More generally, if $\left(E, x_{0}, x_{1}, q, t\right)$ is a point of $\mathcal{E}^{4}$ over some scheme $S$, then we have an isomorphism $\left.\left.\Gamma \mathcal{S}_{x_{0} ; q, t}^{(n)}\right|_{\mathbb{Z}(s+f)} \cong \Gamma \mathcal{S}_{x_{1} ; q, t}^{(n)}\right|_{\mathbb{Z}(s+f)}$ defined Zariski locally on $S$ as long as $x_{1}-x_{0} \in 3 E(S)$. Without this assumption, there may very well be no such isomorphism; indeed for $n=1, q=0$, these are essentially the homogeneous coordinate rings of the embeddings of $C$ via $\left[x_{0}\right]+2[0]$ and $\left[x_{1}\right]+2[0]$.

Remark 8.5. When $c \in E[3]$, this isomorphism becomes an automorphism, but is quite nontrivial. 
The most significant consequence is the following. Note here that we are, as usual, taking global sections before passing to fibers.

Theorem 8.6. For any $v \in \mathbb{Z}\left\langle s, f, e_{1}, \ldots, e_{m}\right\rangle$, there is a codimension $\geq 2$ subscheme of parameter space on the complement of which $\Gamma \mathcal{S}_{\eta^{\prime}, x_{1}, \ldots, x_{m} ; q, t}^{(n)}(0, v)$ is flat and the map to meromorphic difference operators is injective on fibers.

Proof. Applying the Fourier transform has no effect on flatness (since it is an isomorphism), and the Fourier transform will be constructed via an action on meromorphic difference operators, and thus injectivity on fibers is also preserved. This allows us to reduce to Corollary 7.27, as in [26]. To be precise, let $v=d s+d^{\prime} f-r_{1} e_{1}-\cdots-r_{m} e_{m}$. We may apply a permutation and an even number of elementary transformations to put $v$ into the fundamental chamber for $W\left(D_{m}\right)$. Moreover, if $r_{m}<0$, then we may set it to 0 without changing the sheaf of global sections, and in this way may arrange to have $d \geq r_{1}+r_{2}$ and $r_{1} \geq \cdots \geq r_{m} \geq 0$. If $d^{\prime} \geq d$, then we may apply Corollary 7.27. If $d^{\prime}<0$, then we observe that $\Gamma \mathcal{S}_{\eta^{\prime}, x_{1}, \ldots, x_{m} ; q, t}^{(n)}(0, v)=0$, so the result again follows. Otherwise, we apply the Fourier transform. Since this strictly decreases $d$ but keeps it nonnegative, the claim follows by induction.

Remark. Of course, the codimension $\geq 2$ subscheme is the same as that of the appropriate special case of Corollary 7.27.

Just as elliptic pencils gave rise to integrable systems above, there is something analogous (if slightly weaker) for rational pencils. Call a small category with object set $\mathbb{Z}$ "quasi-abelian" if there is a commutative graded algebra $A$ such that $\operatorname{Hom}(j, k) \cong A[k-j]$ for all $j$, $k$, with composition given by multiplication.

Corollary 8.7. Suppose $v \in \mathbb{Z}\left\langle s, f, e_{1}, \ldots, e_{m}\right\rangle$ is the class of a rational pencil on the rational surface $X_{m}$. Then $\left.\Gamma \mathcal{S}_{\eta^{\prime}, x_{1}, \ldots, x_{m} ; q, t}^{(n)}\right|_{\mathbb{Z} v}$ is quasi-abelian, and the corresponding graded algebra is a free polynomial algebra in $n+1$ generators.

Proof. If $v=f$, this is easy: the global sections of degree $d f$ are just multiplication by $C_{n^{-}}$ invariant sections of the bundle with polarization $d \sum_{i} z_{i}^{2}$, and this is precisely the pullback of $\mathcal{O}_{\mathbb{P}^{n}}(d)$. More generally, it follows from the theory of rational surfaces (see [25]) that $v$ represents a rational pencil iff it is in the orbit of $f$ under the group $W\left(E_{m+1}\right)$ generated by the Fourier transform and $W\left(D_{m}\right)$.

Just as integrable systems lead to natural eigenvalue equations, such "quasi-integrable" systems lead to generalized eigenvalue problems. A generalized eigenfunction of a space $\mathcal{D}$ of operators is a function $f$ such that the image $\mathcal{D} f$ is 1-dimensional. (We then obtain an associated "generalized eigenvalue", namely the point in $\mathbb{P}(\mathcal{D})$ associated to the kernel of the map $D \mapsto D f$ on $\mathcal{D}$.)

Given a quasi-integrable system associated to a rational pencil, we have for each $d$ a map $\phi_{d}$ from $A[1]$ to the space of operators, such that $\phi_{d+1}(y) \phi_{d}(x)=\phi_{d+1}(x) \phi_{d}(y)$. We may then consider for each $d$ the generalized eigenvalue problem associated to $\phi_{d}(A[1])$. For any generalized eigenfunction $f_{d}$ for $\phi_{d}(A[1])$, let $f_{d+1}$ be a nonzero representative of $\phi_{d}(A[1]) f_{d}$. Then for suitable $y \in A[1]$, we have

$$
\phi_{d+1}(x) f_{d+1}=\phi_{d+1}(x) \phi_{d}(y) f_{d}=\phi_{d+1}(y) \phi_{d}(x) f_{d}=\lambda_{d}(x) \phi_{d+1}(y) f_{d+1}
$$

for all $x \in A[1]$, and thus $f_{d+1}$ is a generalized eigenfunction for $\phi_{d+1}(A[1])$. More generally, if $V \subset A[1]$ is such that the corresponding generalized eigenvalue problem for $\phi_{d+1}(V)$ is nondegenerate (i.e., for each point of projective space, the corresponding problem has at most 
1-dimensional solution space), then any generalized eigenfunction $f_{d}$ for $\phi_{d}(V)$ is a generalized eigenfunction for $\phi_{d}(A[1])$, since then $\phi_{d}(y) f_{d}$ is a generalized eigenfunction for $\phi_{d+1}(V)$.

There are two cases of particular interest. In the case $v=s+f-e_{1}-e_{2}, \eta^{\prime}=-(n-1) t-q$, the generators of the quasi-integrable system are operators of the form considered in [23], and the quasi-abelian property turns into the quasi-commutation relation used there. The corresponding generalized eigenvalue problem is precisely the difference equation [23, Proposition 3.9] satisfied by the elliptic interpolation functions. (The interpolation kernel of [27] is also a generalized eigenfunction for essentially the same space of operators, [27, Proposition 3.12].)

The biorthogonal functions of [23, 24] are also generalized eigenfunctions of such a quasiintegrable system, corresponding to $v=2 s+2 f-e_{1}-e_{2}-e_{3}-e_{4}-2 e_{5}$ and $\eta^{\prime}=-(n-1) t-q$. Indeed, the first-order difference operators considered in [24] correspond to products of operators of degrees $s-e_{5}, s+2 f-e_{1}-e_{2}-e_{3}-e_{4}-e_{5}$ and $s+f-e_{i}-e_{j}-e_{5}, 1 \leq i<j \leq 4$, giving rise to 8 operators of degree $2 s+2 f-e_{1}-e_{2}-e_{3}-e_{4}-2 e_{5}$. The biorthogonal functions are generalized eigenfunctions of the span of these 8 operators, and the generalized eigenvalues are all distinct points of $\mathbb{P}^{7}$. It thus follows that any biorthogonal function is a generalized eigenfunction for the full space of operators. (With more effort, one can in fact verify that the generalized eigenvalues are given by suitable specializations of the leading coefficients; this is a consequence in general of the fact that the Fourier transform respects leading coefficients.)

We now turn to constructing the Fourier transform. The traditional approach would be to construct a Fourier transform on the DAHA and then observe that it restricts to a transform on the spherical algebra. One significant issue that arises here is that although we have a reasonable facsimile of a presentation, it is at the level of sheaves, not at the level of global sections, while the Fourier transform does not make sense in terms of sheaves (since it does not preserve multiplication). Furthermore, most of the rank 1 subalgebras we used to generate the DAHA do not have any nontrivial global sections (the leading Bruhat subquotient is a generically nontrivial line bundle of degree 0 in every variable). As a result, it seems unlikely that the Fourier transform on the DAHA (assuming it exists) would have a construction that was significantly simpler than the construction we give in the spherical case. Beyond that, there is another issue: as we discussed above, the description of the rank 1 DAHA via a Morita equivalence to the spherical algebra strongly suggests that the Fourier transform only exists for the noncompact version of the DAHA. In other words, the Fourier transform on the DAHA would not respect the filtration by degree; since this filtration comes from the Bruhat filtration, the latter also could not be preserved. As a result, even having a Fourier transform for the DAHA would not be enough to prove the theorem; one also needs to understand why the spherical version is triangular!

We thus wish an approach that works directly with the spherical algebra. Note that since the action of the Fourier transform on objects preserves $\mathbb{Z}\langle s, f\rangle$, the Fourier transform for $m>0$ restricts to a transform of the same sort for $m=0$. Moreover, since every Hom sheaf is contained in one of degree in $\mathbb{Z}\langle s, f\rangle$, it suffices to specify how the transform acts on such sheaves and show that it preserves the various subsheaves of interest. We thus focus our initial attention on the case $m=0$.

In the univariate setting, the Fourier transform was easy to construct: for generic parameters, one can give an explicit presentation for the category (with generators of degrees $s$ and $f$ ) and this presentation has an obvious symmetry. Moreover, a slightly larger set of elements generates the category even without the genericity condition, and one can determine how the transform must act on those elements by taking a suitable limit.

Although we have analogues of those generators (and will indeed be able to describe their Fourier transforms explicitly), this approach founders in the multivariate setting for two reasons. The first is that the operators of degree $s$ and $f$ do not even come close to generating the category for $n>1$ in general: for $q=0, t=0$, the full category is the bihomogeneous coordinate ring of $\operatorname{Sym}^{n}\left(\mathbb{P}^{1} \times \mathbb{P}^{1}\right)$, while the elements of degrees $s$ and $f$ lie in the subring corresponding to 
the quotient $\mathbb{P}^{n} \times \mathbb{P}^{n}$. If we include elements of degree $s+f$, the situation is somewhat better (we will see that these come close enough to generating to be useful), but this only forces us to confront the fact that we have absolutely no understanding of the relations satisfied by these elements.

As a result, we will need some way to construct the Fourier transform which is explicitly a homomorphism. We will do this by constructing a transform on a much larger algebra of operators, and then show that it preserves the particular subspace we care about. The simplest way to construct a homomorphism on a category of operators is to apply a gauge transformation: assign an operator to each object and apply the associated quasi-conjugation.

The first step in constructing such operators is to determine on what spaces they act, and thus we need to think a bit about where our existing operators act. Define a family of (gerbe) polarizations

$$
P_{d}\left(\eta^{\prime} ; q, t\right):=-\left((n-1) t-(d-1) q+\eta^{\prime}\right) \sum_{i} z_{i}^{2} / q .
$$

If $F$ is the product of a $\Gamma_{q}$ symbol with polarization $P_{d_{1}^{\prime}-d_{1}}\left(\eta^{\prime} ; q, t\right)$ and a rational function on $\mathcal{E}^{n}$, then we can apply any global section of a fiber of $\mathcal{S}_{\eta^{\prime} ; q, t}^{(n)}\left(d_{1} s+d_{1}^{\prime} f, d_{2} s+d_{2}^{\prime} f\right)$ and the result will be a rational function times a $\Gamma_{q}$ symbol with polarization $P_{d_{2}^{\prime}-d_{2}}\left(\eta^{\prime} ; q, t\right)$.

Thus the Fourier transform should be given by operators that take functions with polarization $P_{d}\left(\eta^{\prime} ; q, t\right)$ to functions with polarization $P_{-d}\left(-\eta^{\prime} ; q, t\right)$, or equivalently take $P_{0}\left(\eta^{\prime}+d q ; q, t\right)$ to $P_{0}\left(-\eta^{\prime}-d q ; q, t\right)$. There are issues in general, but there is one important case in which operators of this form do indeed exist. Indeed, the simplest way to obtain an operator mapping $P_{d}(0 ; q, t)$ to $P_{-d}(0 ; q, t)$ would be to take a global section of $\mathcal{S}_{0 ; q, t}^{(n)}(d f, d s)$, assuming such a global section exists.

For $d=1$, this is not too difficult to control, and indeed we can understand global sections of order 1 in general.

Lemma 8.8. For any point of $\mathcal{E}^{3}$, the corresponding fiber of $\mathcal{S}_{\eta^{\prime} ; q, t}^{(n)}\left(0, s+d^{\prime} f\right)$ is spanned by operators of the form

$$
\begin{aligned}
D_{q}^{(n)} & \left(u_{0}, u_{1}, \ldots, u_{2 d^{\prime}+1} ; t\right) \\
& =\sum_{\sigma \in\{ \pm 1\}^{n}} \prod_{1 \leq i \leq n} \frac{\prod_{0 \leq r<2 d^{\prime}+2} \vartheta\left(u_{r}+\sigma_{i} z_{i}\right)}{\vartheta\left(2 \sigma_{i} z_{i}\right)} \prod_{1 \leq i<j \leq n} \frac{\vartheta\left(t+\sigma_{i} z_{i}+\sigma_{j} z_{j}\right)}{\vartheta\left(\sigma_{i} z_{i}+\sigma_{j} z_{j}\right)} \prod_{1 \leq i \leq n} T_{i}^{\sigma_{i} / 2},
\end{aligned}
$$

with $u_{0}+\cdots+u_{2 d^{\prime}+1}=q+\eta^{\prime}$.

Proof. The interval $[\leq(1 / 2, \ldots, 1 / 2)]$ in the Bruhat order consists of a single double coset, and thus the space of global sections is (up to multiplication by an explicit product of $\vartheta$ functions) the space of $S_{n}$-invariant sections of the appropriate line bundle. That space is spanned by products of the form $\prod_{1 \leq i \leq n} f\left(z_{i}\right)$, where $f$ is a section of the corresponding line bundle on $\mathcal{E}$, and any such section can be factored into $\vartheta$ functions.

Proposition 8.9. For any $d \geq 0$, the space of global sections of $\mathcal{S}_{0 ; q, t}^{(n)}(d f, d s)$ is 1 -dimensional.

Proof. We proceed by induction in $d$, with the case $d=0$ being obvious. Suppose we are given a nonzero global section $D_{q, t}^{(n)}(d) \in \mathcal{S}_{0 ; q, t}^{(n)}(d f, d s)$. Then for any $u, v$, the operator

$$
\begin{aligned}
D_{q}^{(n)}((d+1) q / 2 \pm u ; t) D_{q, t}^{(n)}(d) & \prod_{1 \leq i \leq n} \vartheta\left(z_{i} \pm v\right) \\
& -D_{q}^{(n)}((d+1) q / 2 \pm v ; t) D_{q, t}^{(n)}(d) \prod_{1 \leq i \leq n} \vartheta\left(z_{i} \pm u\right)
\end{aligned}
$$


is a section of $\mathcal{S}_{0 ; q, t}^{(n)}((d-1) f,(d+1) s)$. Moreover, we know the leading coefficient of $D_{q, t}^{(n)}(d)$ up to a scalar multiple, and may therefore verify that both operators have the same leading coefficient. Since every subquotient below the top of the corresponding univariate vector bundle has negative degree, none of the multivariate subquotients below the top have polarizations represented by positive semidefinite matrices. Thus none of those subquotients have any global sections, let alone symmetric ones. It follows that a section of $\mathcal{S}_{0 ; q, t}^{(n)}((d-1) f,(d+1) s)$ with vanishing leading coefficient must in fact be 0 , and thus

$$
D_{q}^{(n)}((d+1) q / 2 \pm u ; t) D_{q, t}^{(n)}(d) \prod_{1 \leq i \leq n} \vartheta\left(z_{i} \pm v\right)=D_{q}^{(n)}((d+1) q / 2 \pm v ; t) D_{q, t}^{(n)}(d) \prod_{1 \leq i \leq n} \vartheta\left(z_{i} \pm u\right) .
$$

Equivalently,

$$
D_{q}^{(n)}((d+1) q / 2 \pm u ; t) D_{q, t}^{(n)}(d) \prod_{1 \leq i \leq n} \vartheta\left(z_{i} \pm u\right)^{-1}
$$

is independent of $u$. In particular, the apparent $u$-dependent poles of this product of operators are not, in fact, singularities, and thus this gives a section of $\mathcal{S}_{0 ; q, t}^{(n)}((d+1) f,(d+1) s)$ as required. That this is the only global section up to scalar multiples follows by observing that again all Bruhat subquotients below the top have indefinite polarizations, while the top subquotient is trivial.

Following the above proof, we define $D_{q, t}^{(n)}(d)$ by the recurrence

$$
D_{q, t}^{(n)}(d+1)=D_{q}^{(n)}((d+1) q / 2 \pm u ; t) D_{q, t}^{(n)}(d) \prod_{1 \leq i \leq n} \vartheta\left(z_{i} \pm u\right)^{-1},
$$

with base case $D_{q, t}^{(n)}(0)=1$. (Note that $D_{q, t}^{(n)}(1)=D_{q}^{(n)}(; t)$.) Equivalently, $D_{q, t}^{(n)}(d)$ is the unique global section of $\mathcal{S}_{0 ; q, t}^{(n)}(d f, d s)$ with leading term

$$
\prod_{1 \leq i<j \leq n} \frac{\Gamma_{q}\left(d q+t-z_{i}-z_{j}\right)}{\Gamma_{q}\left(t-z_{i}-z_{j}\right)} \prod_{1 \leq i \leq j \leq n} \frac{\Gamma_{q}\left(-z_{i}-z_{j}\right)}{\Gamma_{q}\left(d q-z_{i}-z_{j}\right)} \prod_{1 \leq i \leq n} T_{i}^{-d / 2} .
$$

Since translation by $s+f$ does not change the parameters, this also gives a global section of $\mathcal{S}_{0 ; q, t}^{(n)}\left(d_{0}(s+f)+d f, d_{0}(s+f)+d s\right)$ for any $d_{0}$.

Corollary 8.10. If $q$ has exact order $d$, then

$$
D_{q, t}^{(n)}(d)=\sum_{\sigma \in\{ \pm 1\}^{n}} \prod_{1 \leq i \leq n} \frac{1}{\vartheta\left(2 \sigma_{i} z_{i} ; q\right)_{d}} \prod_{1 \leq i<j \leq n} \frac{\vartheta\left(t+\sigma_{i} z_{i}+\sigma_{j} z_{j} ; q\right)_{d}}{\vartheta\left(\sigma_{i} z_{i}+\sigma_{i} z_{j} ; q\right)_{d}} \prod_{1 \leq i \leq n} T_{i}^{-d / 2} .
$$

Proof. By Theorem 7.15, the center of the given spherical algebra is itself a spherical algebra with $q=0$. In particular, the center contains a nonzero element mapping $f$ to $s$, which becomes an element of $\mathcal{S}_{0 ; q, t}^{(n)}(d f, d s)$ under the isomorphism. By uniqueness, this element is proportional to $D_{q, t}^{(n)}(d)$. For this element to be central, every shift that appears must be congruent to $-d / 2$ modulo $d$. There is only one $C_{n}$-orbit of shifts that survives, so that we may recover all coefficients from the leading coefficient, and obtain the stated formula.

Remark. If we gauge by a product of gamma functions before specializing $q$ (à la an elementary transformation), the interior coefficients will still vanish, giving central sections of degree $d\left(s+d^{\prime} f\right)$ for any $d^{\prime}$, and establishing that any element of the center with degree of the given form is obtained in this way. Since the Fourier transform respects leading coefficients, we can compute how it acts on central elements of degree $d s, d f, d(s+f)$, and thus conclude (following the argument below) that the Fourier transform respects the isomorphism of Theorem 7.15. 
The following result shows that these operators indeed behave like Fourier transforms.

Proposition 8.11. We have the operator relations

$$
\begin{aligned}
& D_{q}^{(n)}((d+1) q / 2 \pm u ; t) D_{q, t}^{(n)}(d)=D_{q, t}^{(n)}(d+1) \prod_{1 \leq i \leq n} \vartheta\left(z_{i} \pm u\right), \\
& D_{q, t}^{(n)}(d) D_{q}^{(n)}(-d q / 2 \pm u ; t)=\prod_{1 \leq i \leq n} \vartheta\left(z_{i} \pm u\right) D_{q, t}^{(n)}(d+1),
\end{aligned}
$$

and, if $u_{0}+u_{1}+u_{2}+u_{3}=(d+1) q$,

$$
D_{q, t}^{(n)}(d) D_{q}^{(n)}\left(u_{0}, u_{1}, u_{2}, u_{3} ; t\right)=D_{q}^{(n)}\left(u_{0}+d q / 2, u_{1}+d q / 2, u_{2}+d q / 2, u_{3}+d q / 2 ; t\right) D_{q, t}^{(n)}(d) .
$$

Proof. In each case, both sides are sections of the same Hom sheaf of $\mathcal{S}_{0 ; q, t}^{(n)}$ with the same leading coefficient, and only the top subquotient has positive semidefinite polarization.

It turns out that if we adjoined formal inverses of the operators $D_{q, t}^{(n)}(d)$ and declared them to be $D_{q, t}^{(n)}(-d)$, then the result would indeed define a Fourier transform on a certain subcategory of the category with $\eta^{\prime}=0$ (in which the Hom sheaves of degree $d s+d^{\prime} f$ for $d>d^{\prime}$ are replaced by the images under the Fourier transform of the Hom sheaves of degree $d^{\prime} s+d f$ ). Proving this directly is somewhat tricky, however, as unlike in the univariate setting, there does not appear to be a readily accessible test for right divisibility by $D_{q, t}^{(n)}(d)$. And, of course, even using the translation symmetry, this would at best give us a transform for $\eta^{\prime} \in \mathbb{Z} q$, which is especially weak when $q$ is torsion.

The key idea for proceeding further is that the relation

$$
D_{q, t}^{(n)}(d) D_{q}^{(n)}(-d q / 2 \pm u ; t)=\prod_{1 \leq i \leq n} \vartheta\left(z_{i} \pm u\right) D_{q, t}^{(n)}(d+1)
$$

gives us a system of recurrences that we can use to solve for the coefficients of $D_{q, t}^{(n)}(d)$. Indeed, it follows from this relation that

$$
\left.D_{q, t}^{(n)}(d) D_{q}^{(n)}(-d q / 2 \pm u ; t)\right|_{u=z_{i}}=0
$$

for $1 \leq i \leq n$. Since the operators are symmetric, let us consider the specialization $u=z_{n}$. The coefficient of $\prod_{i} T_{i}^{k_{i}-(d+1) / 2}$ in this specialized operator is a linear combination of the left coefficients of $\prod_{i} T_{i}^{l_{i}-d / 2}$ in $D_{q, t}^{(n)}(d)$ for $\max \left(k_{i}-1,0\right) \leq l_{i} \leq k_{i}$. The coefficient in this linear combination for $\vec{l}=\vec{k}$ is

$$
\frac{\prod_{1 \leq i \leq n} \vartheta\left(-k_{i} q+z_{n}-z_{i}, k_{i} q+z_{i}+z_{n}\right) \prod_{1 \leq i<j \leq n} \vartheta\left(t+d q-\left(k_{i}+k_{j}\right) q-z_{i}-z_{j}\right)}{\prod_{1 \leq i \leq j \leq n} \vartheta\left(d q-\left(k_{i}+k_{j}\right) q-z_{i}-z_{j}\right)}
$$

and thus we can solve for the coefficient of $\prod_{i} T_{i}^{k_{i}-d / 2}$ in $D_{q, t}^{(n)}(d)$, at least generically. In fact, we find the only difficulty arises when $\vartheta\left(-k_{n} q\right)=0$, so if $q$ is not torsion and $\vec{k} \neq 0$, there will always be one of the $n$ specializations that allows us to solve for the coefficient of $\prod_{i} T_{i}^{k_{i}-d / 2}$ in terms of coefficients of terms which are smaller in dominance order. In other words, $D_{q, t}^{(n)}(d)$ is determined by the given relation along with the choice of leading coefficient.

The fact that we can control coefficients near the leading coefficients suggests a way to proceed further: take an appropriate completion! Define a nonarchimedean metric on the $\mathbb{Z} / 2 \mathbb{Z}$-graded algebra $k(X)\left[T_{1}, \ldots, T_{n}, \prod_{i} T_{i}^{-1 / 2}\right]$ by

$$
\left|\sum_{\vec{k}} c_{k} \prod_{i} T_{i}^{k_{i}}\right|:=\max _{\vec{k}: c_{k} \neq 0} \exp \left(-\sum_{i} k_{i}\right) \text {. }
$$


We call an element of the corresponding completion a formal difference operator. We in particular denote the completion of the subalgebra $k(X)\left[T_{1}, \ldots, T_{n}\right]$ by $k(X)\left[\left[T_{1}, \ldots, T_{n}\right]\right]$. This construction of course applies equally well to the case of twisted difference operators, or even to the corresponding category in which the objects are polarizations $P_{d}\left(\eta^{\prime} ; q, t\right)$ with fixed $\eta^{\prime}$. We will mostly suppress the twisting from the notation.

The major advantage of formal difference operators is that the ring has a large number of units. Indeed, the usual argument for inverting a commutative formal power series with invertible constant term applies equally well in the noncommutative setting to give the following.

Proposition 8.12. If $D \in k(X)\left[\left[T_{1}, \ldots, T_{n}\right]\right]$ has nonzero constant term, then $D$ is a unit.

Since $\prod_{i} T_{i}^{-1 / 2}$ is also clearly invertible, we find that any of the operators $D_{q, t}^{(n)}(d)$ are invertible as formal operators. In fact, in the ring of formal operators we can solve for $D_{q, t}^{(n)}(d)$ in terms of $D_{q, t}^{(n)}(d+1)$ and in this way define $D_{q, t}^{(n)}(d)$ for $d<0$. We then find by an easy induction that $D_{q, t}^{(n)}(-d)=D_{q, t}^{(n)}(d)^{-1}$.

In addition to these inner automorphisms, we also have automorphisms coming from gauging by $\Gamma_{q}$ symbols and translations on $E$. Let $T_{\omega}(c)$ denote the translation of all variables by $c$, so that $T_{\omega}(q / 2)=\prod_{1 \leq i \leq n} T_{i}^{1 / 2}$. Then for any $\Gamma_{q}$ symbol $\Gamma$ of polarization

$$
\left(\eta^{\prime}-\eta^{\prime \prime}\right) \sum_{i} z_{i}^{2} / q+2\left((n-1) t+q+\eta^{\prime}\right) c \sum_{i} z_{i} / q
$$

there is an induced isomorphism

$$
D \mapsto \Gamma T_{\omega}(c) D T_{\omega}(-c) \Gamma^{-1}
$$

from $\operatorname{End}\left(P_{0}\left(\eta^{\prime} ; q, t\right)\right)^{0}$ (the subspace involving only integer powers of $\left.T_{i}\right)$ to $\operatorname{End}\left(P_{0}\left(\eta^{\prime \prime} ; q, t\right)\right)^{0}$. With this in mind, we define a "formal gauging operator" from $P_{0}\left(\eta^{\prime} ; q, t\right)$ to $P_{0}\left(\eta^{\prime \prime} ; q, t\right)$ to be an object of the form

$$
\Gamma T_{\omega}(c) D,
$$

where $D$ is a unit in the endomorphism ring. The "leading term" of such an operator is the formal symbol $\Gamma T_{\omega}(c) f$, where $f$ is the constant term of $D$. The formal gauging operators form a group, with a natural subgroup consisting of elements of the form $\Theta T_{\omega}(k q / 2) D$, where $\Theta$ is a product of $\vartheta$ symbols (i.e., of invertible formal difference operators). If $G_{1}, G_{2}$ are formal gauging operators such that $G_{1} G_{2}^{-1}$ lies in the subgroup of formal difference operators, then for any formal difference operator $D$ with only integer shifts (and with coefficients having appropriate polarizations), $G_{1} D G_{2}^{-1}:=\left(G_{1} G_{2}^{-1}\right) G_{2} D G_{2}^{-1}$ will again be a formal difference operator. This extends to the half-integer case by writing $D=T_{\omega}(q / 2) D^{\prime}$ and $G_{1} D G_{2}^{-1}:=\left(G_{1} T_{\omega}(q / 2) G_{2}^{-1}\right) G_{2} D^{\prime} G_{2}^{-1}$. In either case, the operation clearly respects multiplication as long as the gauging operators match up.

Proposition 8.13. There is a unique family of formal gauging operators $\mathcal{D}_{q, t}^{(n)}(c)$ from $P_{0}(2 c ; q, t)$ to $P_{0}(-2 c ; q, t)$ with leading term

$$
\prod_{1 \leq i \leq j \leq n} \frac{\Gamma_{q}\left(-z_{i}-z_{j}\right)}{\Gamma_{q}\left(-2 c-z_{i}-z_{j}\right)} \prod_{1 \leq i<j \leq n} \frac{\Gamma_{q}\left(t-2 c-z_{i}-z_{j}\right)}{\Gamma_{q}\left(t-z_{i}-z_{j}\right)} T_{\omega}(c)
$$

such that $\mathcal{D}_{q, t}^{(n)}(-d q / 2)=D_{q, t}^{(n)}(d)$ for all $d \in \mathbb{Z}$. Moreover, if one divides any coefficient of $\mathcal{D}_{q, t}^{(n)}(c)$ by the leading term, then the only z-independent poles of the resulting meromorphic section on $\mathcal{E}^{n+3}$ are along hypersurfaces for which $q$ is torsion. 
Proof. If such a family of operators exists, then it must satisfy

$$
\left.\mathcal{D}_{q, t}^{(n)}(c) D_{q}^{(n)}(c \pm u ; t)\right|_{u=z_{i}}=0
$$

for $1 \leq i \leq n$. This gives an algebraic (and triangular) system of equations for the coefficients of $\mathcal{D}_{q, t}^{(n)}(c)$ which we have already seen has at most one solution (and if it has a solution, the only $z$-independent poles are where $q$ is torsion). Since it has a solution on the Zariski dense set of divisors $c \in \mathbb{Z} q / 2$, it must have a solution in general.

Remark. For an analytic approach to constructing such operators, see [27].

Since we understand a Zariski dense subset of these operators, we can immediately deduce some relations.

Proposition 8.14. The operators $\mathcal{D}_{q, t}^{(n)}(c)$ satisfy the operator identities

$$
\begin{aligned}
& D_{q}^{(n)}(-c \pm u ; t) \mathcal{D}_{q, t}^{(n)}(c+q / 2)=\mathcal{D}_{q, t}^{(n)}(c) \prod_{1 \leq i \leq n} \vartheta\left(z_{i} \pm u\right), \\
& \mathcal{D}_{q, t}^{(n)}(c) D_{q}^{(n)}(c \pm u ; t)=\prod_{1 \leq i \leq n} \vartheta\left(z_{i} \pm u\right) \mathcal{D}_{q, t}^{(n)}(c-q / 2),
\end{aligned}
$$

and, if $u_{0}+u_{1}+u_{2}+u_{3}=q+2 c$,

$$
\mathcal{D}_{q, t}^{(n)}(c) D_{q}^{(n)}\left(u_{0}, u_{1}, u_{2}, u_{3} ; t\right)=D_{q}^{(n)}\left(u_{0}-c, u_{1}-c, u_{2}-c, u_{3}-c ; t\right) \mathcal{D}_{q, t}^{(n)}(c) .
$$

We also note the following fact, generalizing the first two identities.

Proposition 8.15. The operators $\mathcal{D}_{q, t}^{(n)}(c)$ satisfy the operator identity

$$
\prod_{1 \leq i \leq n} \frac{\Gamma_{q}\left(t_{0}-d \pm z_{i}\right)}{\Gamma_{q}\left(t_{0}+d \pm z_{i}\right)} \mathcal{D}_{q, t}^{(n)}(c+d) \prod_{1 \leq i \leq n} \frac{\Gamma_{q}\left(t_{0}-c \pm z_{i}\right)}{\Gamma_{q}\left(t_{0}+c \pm z_{i}\right)}=\mathcal{D}_{q, t}^{(n)}(c) \prod_{1 \leq i \leq n} \frac{\Gamma_{q}\left(t_{0}-c-d \pm z_{i}\right)}{\Gamma_{q}\left(t_{0}+c+d \pm z_{i}\right)} \mathcal{D}_{q, t}^{(n)}(d)
$$

In particular, $\mathcal{D}_{q, t}^{(n)}(c)^{-1}=\mathcal{D}_{q, t}^{(n)}(-c)$.

Proof. Consider the composition

$$
\prod_{1 \leq i \leq n} \frac{\Gamma_{q}\left(t_{0}+d \pm z_{i}\right)}{\Gamma_{q}\left(t_{0}-d \pm z_{i}\right)} \mathcal{D}_{q, t}^{(n)}(c) \prod_{1 \leq i \leq n} \frac{\Gamma_{q}\left(t_{0}-c-d \pm z_{i}\right)}{\Gamma_{q}\left(t_{0}+c+d \pm z_{i}\right)} \mathcal{D}_{q, t}^{(n)}(d) \prod_{1 \leq i \leq n} \frac{\Gamma_{q}\left(t_{0}+c \pm z_{i}\right)}{\Gamma_{q}\left(t_{0}-c \pm z_{i}\right)} .
$$

If we substitute

$$
\mathcal{D}_{q, t}^{(n)}(d)=D_{q}^{(n)}\left(-d \pm\left(t_{0}-c\right) ; t\right) \mathcal{D}_{q, t}^{(n)}(d+q / 2) \prod_{1 \leq i \leq n} \vartheta\left(z_{i} \pm\left(t_{0}-c\right)\right)^{-1},
$$

then apply the easy relation

$$
\begin{aligned}
& \prod_{1 \leq i \leq n} \frac{\Gamma_{q}\left(t_{0}-c-d \pm z_{i}\right)}{\Gamma_{q}\left(t_{0}+c+d \pm z_{i}\right)} D_{q}^{(n)}\left(c-d-t_{0}, t_{0}-c-d ; t\right) \\
& =D_{q}^{(n)}\left(c-d-t_{0}, t_{0}+c+d ; t\right) \prod_{1 \leq i \leq n} \frac{\Gamma_{q}\left(t_{0}+q / 2-c-d \pm z_{i}\right)}{\Gamma_{q}\left(t_{0}+q / 2+c+d \pm z_{i}\right)}
\end{aligned}
$$

we can combine the two operators:

$$
\mathcal{D}_{q, t}^{(n)}(c) D_{q}^{(n)}\left(c \pm\left(t_{0}+d\right) ; t\right)=\prod_{1 \leq i \leq n} \vartheta\left(z_{i} \pm\left(t_{0}+d\right)\right) \mathcal{D}_{q, t}^{(n)}(c-q / 2),
$$


and find that the result simplifies to the case $\left(c, d, t_{0}\right) \mapsto\left(c-q / 2, d+q / 2, t_{0}+q / 2\right)$ of the above composition. In other words, the given operator is invariant under such translations, so by density is invariant under any translation $\left(c, d, t_{0}\right) \mapsto\left(c-u, d+u, t_{0}+u\right)$. Taking $u=c$ gives

$$
\prod_{1 \leq i \leq n} \frac{\Gamma_{q}\left(t_{0}+2 c+d \pm z_{i}\right)}{\Gamma_{q}\left(t_{0}-d \pm z_{i}\right)} \mathcal{D}_{q, t}^{(n)}(0) \prod_{1 \leq i \leq n} \frac{\Gamma_{q}\left(t_{0}-d \pm z_{i}\right)}{\Gamma_{q}\left(t_{0}+2 c+d \pm z_{i}\right)} \mathcal{D}_{q, t}^{(n)}(c+d)=\mathcal{D}_{q, t}^{(n)}(c+d)
$$

since $\mathcal{D}_{q, t}^{(n)}(0)=1$.

Remark 8.16. Compare the proof of [23, Theorem 4.1]. The similarity in arguments is not at all a coincidence: The analytic construction of $\mathcal{D}_{q, t}^{(n)}(c)$ in terms of the interpolation kernel of [27] implies that one can obtain the elliptic binomial coefficients of [23] as specializations of the coefficients of $\mathcal{D}_{q, t}^{(n)}(c)$, making [23, Theorem 4.1] a (Zariski dense) special case of the above relation.

Remark 8.17. The action of the Fourier transform on objects is a reflection in an appropriate inner product (the intersection form of the surface!), as are the generators of the $W\left(D_{m}\right)$ action. Each generator has a certain action on operators. If one takes into account the action on parameters, the generators are involutions, and all relevant braid relations are satisfied, so that this gives an action of a Coxeter group $W\left(E_{m+1}\right)$ on the base of the family. Showing that this lifts to an action of $W\left(E_{m+1}\right)$ on the actual sheaf categories reduces to verifying lifts of each braid relation, and the only nontrivial such lift reduces to the above identity.

We thus define a Fourier transform on formal difference operators in the following way. If the formal operator $D$ maps the polarization $P_{0}(2 c ; q, t)$ to the polarization $P_{0}\left(2 c^{\prime} ; q, t\right)$, then its Fourier transform $\hat{D}$ is the operator

$$
\mathcal{D}_{q, t}^{(n)}\left(c^{\prime}\right) D \mathcal{D}_{q, t}^{(n)}(-c)
$$

mapping $P_{0}(-2 c ; q, t)$ to $P_{0}\left(-2 c^{\prime} ; q, t\right)$. There is some choice here (since the polarizations only depend on $\left.2 c, 2 c^{\prime}\right)$, but luckily it is not particularly serious.

Lemma 8.18. If $\tau$ is a 2-torsion point, then

$$
\mathcal{D}_{q, t}^{(n)}(c+\tau)=T_{\omega}(\tau) \mathcal{D}_{q, t}^{(n)}(c)=\mathcal{D}_{q, t}^{(n)}(c) T_{\omega}(\tau) .
$$

Proof. Indeed, the recurrence we used to solve for the coefficients of $\mathcal{D}_{q, t}^{(n)}(c)$ is equivariant under translation by 2-torsion.

For our purposes, we will always be working in the subcategory with objects $P_{0}\left(-d q+\eta^{\prime} ; q, t\right)$, and will take $c, c^{\prime}$ in the Fourier transform to be the appropriate linear combination of $q / 2$ and some fixed $\eta^{\prime} / 2$. We have, of course, already computed some instances of the Fourier transform:

$$
\begin{gathered}
\prod_{1 \leq i \leq n} \vartheta\left(z_{i} \pm u\right) \mapsto D_{q}^{(n)}(q / 2-c \pm u ; t), \\
D_{q}^{(n)}(c+q / 2 \pm u ; t) \mapsto \prod_{1 \leq i \leq n} \vartheta\left(z_{i} \pm u\right),
\end{gathered}
$$

and

$$
D_{q}^{(n)}\left(u_{0}, u_{1}, u_{2}, q+2 c-u_{0}-u_{1}-u_{2} ; t\right) \mapsto D_{q}^{(n)}\left(u_{0}-c, u_{1}-c, u_{2}-c, q+c-u_{0}-u_{1}-u_{2} ; t\right),
$$

where in each case the input is a (general) section of $\mathcal{S}_{2 c ; q, t}^{(n)}$ starting from the 0 object, of degree $f, s$, and $s+f$ respectively. 
Theorem 8.19. If $c^{\prime}-c$ is an integer multiple of $q / 2$, then the Fourier transform is holomorphic; that is, the Fourier transform of any holomorphic family of operators is a holomorphic family of operators.

Proof. The only issue is when $q$ is torsion, as otherwise both $\mathcal{D}_{q, t}^{(n)}(-c)$ and $\mathcal{D}_{q, t}^{(n)}\left(c^{\prime}\right)$ are holomorphic (in the sense that they have no $z$-independent poles other than those for $q$ torsion).

Consider a multiplication operator $h$. If this is $C_{n}$-invariant, we can express it as a ratio of holomorphic $C_{n}$-invariant theta functions. The algebra of such theta functions is generated by functions $\prod_{1 \leq i \leq n} \vartheta\left(u \pm z_{i}\right)=(-1)^{n} \prod_{1 \leq i \leq n} \vartheta\left(z_{i} \pm u\right)$, and thus any holomorphic family of $C_{n}$-invariant functions $h$ has holomorphic Fourier transform. (The leading term of the Fourier transform of an operator is easy to determine, so we find that the Fourier transform of the denominator is indeed invertible.)

Now, let $h$ be a general multiplication operator. To show that $\hat{h}$ is holomorphic, we need to show that every coefficient is holomorphic. The coefficient of $\prod_{i} T_{i}^{k_{i}}$ has denominator dividing $\prod_{1 \leq j \leq \max \left(k_{1}, \ldots, k_{n}\right)} \vartheta(j q)$, and by Hartog's lemma it suffices to prove that the coefficient is holomorphic at the generic point of every component of the corresponding divisor. Each coefficient is a finite linear combination of shifts of $h$, and we are evaluating it at a point with generic $\left(z_{1}, \ldots, z_{n}\right)$. In particular, none of the points where we are evaluating $h$ are in the same $C_{n}$ orbit (though we may be hitting the same point multiple times). It follows that there exists a $C_{n}$-invariant function $g$ such that the corresponding sum for $h-g$ is holomorphic: simply take $g$ to be a very good approximation near the points where $h$ is being evaluated. Since $\hat{g}$ is holomorphic and this coefficient of the Fourier transform of $h-g$ is holomorphic, it follows that the given coefficient of $\hat{h}$ is holomorphic as required.

Now, let $D$ be an operator of the form $D_{q}^{(n)}(c+q / 2 \pm u ; t)$, which again has a holomorphic Fourier transform. If $q \neq 0$, then the space of operators $k(X) D_{q}^{(n)}(c+q / 2 \pm u ; t) k(X)$ is a $2^{n}$-dimensional vector space on the left. Indeed, each of the $2^{n}$ shifts that appear induce different automorphisms of $k(X)$. It follows that any element of that space has holomorphic Fourier transform (except possibly where $q=0$ ). Since that space contains elements $\propto \prod_{i} T_{i}^{ \pm 1 / 2}$ for every combination of signs, we have proved holomorphy of the Fourier transform on a set of (topological) generators of the ring of twisted formal difference operators. The Fourier transform is continuous with respect to the nonarchimedean metric, so the result follows in general.

It remains to consider the case $q=0$. This splits into two components, depending on whether $q / 2=0$ or $q / 2$ is nontrivial 2 -torsion. The latter case reduces to the first, however, since everything is invariant under translation by 2 -torsion. We may thus restrict our attention to the local ring at the generic point with $q / 2=0$. In that case, the special fiber of the ring of twisted formal difference operators is abelian, since all shifts are trivial. As a result, the algebra over the local ring picks up an additional (Poisson bracket) operation on operators: $\left(D_{1}, D_{2}\right) \mapsto\left(D_{1} D_{2}-D_{2} D_{1}\right) / \pi$, where $\pi$ is a uniformizer. This takes any pair of holomorphic families of operators to a holomorphic family of operators, and the Fourier transform respects this operation. We may thus use this operation to construct operators with known holomorphic Fourier transform. It turns out that the usual proof of independence of automorphisms of fields can be expressed in terms of this operation, and thus we still obtain the full $2^{n}$-dimensional space of operators.

Remark. The proof for $q=0$ is of course based on the standard fact that an automorphism of a family of noncommutative algebras preserves the induced Poisson structure on any commutative fiber.

Of course, the algebra of formal difference operators is far too large, and doesn't even have an action of $C_{n}$ (as it preserves neither the metric nor the topology). So we need to show that 
the operators we care about map to operators which not only have finite support, but have $C_{n}$ symmetry. Luckily, this is a closed condition, so it suffices to prove it generically.

Lemma 8.20. On the generic fiber, the $\mathbb{Z} / 2 \mathbb{Z}$-graded algebra $\bigcup_{d} \mathcal{S}_{2 c ; 0,0}^{(n)}(0, d(s+f))$ is generated by $\mathcal{S}_{2 c ; 0,0}^{(n)}(0, s+f)$.

Proof. In fact, we claim that for $d \gg 0, \mathcal{S}_{2 c ; 0,0}^{(n)}(0, d(s+f))$ is spanned by products of $d$ elements of $\mathcal{S}_{2 c ; 0,0}^{(n)}(0, s+f)$. Since this contains the spaces for all smaller $d$ of the same parity, the result will immediately follow.

Since this graded algebra is the homogeneous coordinate ring of $\operatorname{Sym}^{n}\left(\mathbb{P}^{1} \times \mathbb{P}^{1}\right)$, what we are in fact claiming is that the ample bundle $\operatorname{Sym}^{n}\left(\mathcal{O}_{\mathbb{P}^{1} \times \mathbb{P}^{1}}(1)\right)$ is very ample. In general, it follows from [4, Section 1.3] that over any field of characteristic 0, $\operatorname{Sym}^{n}\left(\mathcal{O}_{\mathbb{P} m}(1)\right)$ is very ample on $\operatorname{Sym}^{n}\left(\mathbb{P}^{m}\right)$, and thus the same holds for the symmetric power of any closed subscheme of $\mathbb{P}^{m}$.

Remark. It is likely that this fails in small characteristic. It is certainly the case that the line bundle $\operatorname{Sym}^{n}\left(\mathcal{O}_{\mathbb{P}^{m}}(1)\right)$ can fail to be very ample on $\operatorname{Sym}^{n}\left(\mathbb{P}^{m}\right)$; indeed this already happens for $\operatorname{Sym}^{3}\left(\mathbb{P}^{2}\right)$ in characteristic 3. In addition, even in characteristic 0 , the $\mathbb{Z}$-graded algebra is not generated in degree 1 if $n$ is sufficiently large. Indeed, one has $h^{0}\left(\operatorname{Sym}^{n}\left(\mathcal{O}_{\mathbb{P}^{1} \times \mathbb{P}^{1}}(1)\right)\right)=\left(\begin{array}{c}n+3 \\ 3\end{array}\right)$, while $h^{0}\left(\operatorname{Sym}^{n}\left(\mathcal{O}_{\mathbb{P}^{1} \times \mathbb{P}^{1}}(2)\right)\right)=\left(\begin{array}{c}n+8 \\ 8\end{array}\right)$. So for $n \gg 0$, even if we take into account noncommutativity, there are simply not enough sections of degree 1 for their products to account for every section of degree 2 !

Corollary 8.21. For any $d$, the Fourier transform induces an isomorphism of stalks

$$
\Gamma \mathcal{S}_{2 c ; q, t}^{(n)}(0, d(s+f))_{q=t=0} \cong \Gamma \mathcal{S}_{-2 c ; q, t}^{(n)}(0, d(s+f))_{q=t=0} .
$$

Proof. Fix a basis of the global sections of the fiber over the generic point with $q=t=0$. Each such global section can be expressed as a polynomial in sections of degree 1; if we choose an extension to the stalk for each degree 1 operator that appears, then the result will be a basis of the stalk of degree $d$ operators in which every element is a polynomial in first-order operators. It follows that every element of the basis has Fourier transform in $\bigcup_{e \geq 0} \Gamma \mathcal{S}_{-2 c ; q, t}^{(n)}(0, e(s+f))_{q=t=0}$, but the Fourier transform clearly preserves the space of operators $\prod_{i} T_{i}^{-l / 2} k(X)\left[\left[T_{1}, \ldots, T_{n}\right]\right]$ for each $l$, and thus the Fourier transform is actually in $\Gamma \mathcal{S}_{-2 c ; q, t}^{(n)}(0, d(s+f))_{q=t=0}$ as required. The inverse operation is of course just the Fourier transform again.

Corollary 8.22. For any d, the Fourier transform induces a (local) isomorphism of sheaves of categories $\left.\left.\Gamma \mathcal{S}_{2 c ; q, t}^{(n)}\right|_{\mathbb{Z}(s+f)} \cong \Gamma \mathcal{S}_{-2 c ; q, t}^{(n)}\right|_{\mathbb{Z}(s+f)}$.

Proof. The given Hom sheaves of the global section category are flat and the map to difference operators is injective on fibers. We may thus identify sections with holomorphic families of difference operators and apply the Fourier transform to obtain a holomorphic family of formal difference operators. The generic point of this family is a section of the other global section category, and thus the family itself is a section.

Corollary 8.23. For $d \leq d^{\prime}$, the Fourier transform induces a morphism

$$
\Gamma \mathcal{S}_{2 c ; q, t}^{(n)}\left(0, d s+d^{\prime} f\right) \rightarrow \Gamma \mathcal{S}_{-2 c ; q, t}^{(n)}\left(0, d^{\prime} s+d f\right)
$$

Proof. If $d=0$, this is easy, as the algebra is generated in degree 1 , and we know the result there. More generally, given a section $D$ of $\Gamma \mathcal{S}_{2 c ; q, t}^{(n)}\left(0, d s+d^{\prime} f\right)$ and any section $g$ of $\Gamma \mathcal{S}_{-2 c ; q, t}^{(n)}\left(d^{\prime} s+\right.$ 
$\left.d f, d^{\prime} s+d^{\prime} f\right)$, consider the composition $\hat{g} D \in \Gamma \mathcal{S}_{2 c ; q, t}^{(n)}\left(0, d^{\prime} s+d^{\prime} f\right)$, which makes sense since $g$ has degree $\left(d^{\prime}-d\right) f$. Since the Fourier transform is a covariant involution, we find that $\hat{g} D$ has Fourier transform $g \hat{D}$, so that $g \hat{D}$ is a section of $\Gamma \mathcal{S}_{-2 c ; q, t}^{(n)}\left(0, d^{\prime} s+d^{\prime} f\right)$ for any $g$. But this implies that $\hat{D}$ is actually a section of $\Gamma \mathcal{S}_{-2 c ; q, t}^{(n)}\left(0, d^{\prime} s+d f\right)$ as required.

Corollary 8.24. For any $d, d^{\prime}$, the sheaf $\Gamma \mathcal{S}_{2 c ; q, t}^{(n)}\left(0, d s+d^{\prime} f\right)$ is flat and the map to difference operators is injective on fibers. Moreover, the Fourier transform induces an isomorphism

$$
\Gamma \mathcal{S}_{2 c ; q, t}^{(n)}\left(0, d s+d^{\prime} f\right) \cong \Gamma \mathcal{S}_{-2 c ; q, t}^{(n)}\left(0, d^{\prime} s+d f\right)
$$

for all $d, d^{\prime}$.

Proof. We already have flatness if $d<0$ or $d \leq d^{\prime}$, so suppose $d \geq d^{\prime}$. It suffices to show injectivity on fibers, as it implies that any $\mathrm{Tor}_{1}$ of the cokernel is 0 . Thus, let $D \in \Gamma \mathcal{S}_{2 c ; q, t}^{(n)}(0, d s+$ $d^{\prime} f$ ) be a local section such that the corresponding difference operator vanishes on some fiber. Consider the Fourier transform

$$
\Gamma \mathcal{S}_{-2 c ; q, t}^{(n)}\left(0, d^{\prime} s+d f\right) \rightarrow \Gamma \mathcal{S}_{2 c ; q, t}^{(n)}\left(0, d s+d^{\prime} f\right)
$$

The domain is locally free and injective on fibers, and the codomain is at least generically free of the same rank. Since the Fourier transform is invertible at the level of operators, this map is injective, and thus generically an isomorphism. It follows that there is a section $D^{\prime} \in$ $\Gamma \mathcal{S}_{-2 c ; q, t}^{(n)}\left(0, d^{\prime} s+d f\right)$ such that $D-\hat{D}^{\prime}$ is generically 0 . But this, of course, implies that $D^{\prime}=\hat{D}$. In particular, the corresponding fiber of $D^{\prime}$ vanishes, which means that in a suitable local basis we have $D^{\prime}=\sum_{i} c_{i} D_{i}$, in which each $c_{i}$ vanishes on a divisor passing through that fiber. We then have $D=\sum_{i} c_{i} \hat{D}_{i}$ with each $\hat{D}_{i}$ a local section of $\Gamma \mathcal{S}_{2 c ; q, t}^{(n)}\left(0, d s+d^{\prime} f\right)$. It follows that the section corresponding to $D$ vanishes at the fiber, so that injectivity holds.

In particular, the Fourier transform induces a morphism in both directions, and thus gives an isomorphism as required.

To finish the proof of the theorem, we need to show that the transform respects Bruhat order, that it respects the vanishing conditions associated to $x_{1}, \ldots, x_{m}$, and that it commutes with the Selberg adjoint. Each of these have analogous statements for general formal difference operators, and in the first two cases reduce to the fact that (due to continuity) the Fourier transform affects leading coefficients in easy to control ways.

For the Bruhat order, we actually obtain a finer (inclusion) partial order in the formal setting.

Proposition 8.25. Let $D$ be a holomorphic family of formal difference operators from $P_{0}(2 c ; q, t)$ to $P_{0}(2 c+l q ; q, t)$. Let $S \subset \mathbb{Z}^{n} \cup(1 / 2, \ldots, 1 / 2) \mathbb{Z}^{n}$ be the set of vectors $\vec{v}$ such that for some $\vec{k} \in \mathbb{N}^{n}$, the left coefficient of $\prod_{i} T_{i}^{v_{i}-k_{i}}$ is nonzero, and let $\hat{S}$ be the corresponding set for $\hat{D}$. Then $\hat{S}=(l / 2, \ldots, l / 2)+S$.

Proof. Conjugating by $T_{\omega}(c)$ or a $\Gamma_{q}$ symbol has no effect on the support of an operator, and multiplication by $T_{\omega}(l q / 2)$ shifts the support by $(l / 2, \ldots, l / 2)$. The remaining operation consists of left- and right-multiplication by units in $k(X)\left[\left[T_{1}, \ldots, T_{n}\right]\right]$, and this clearly preserves the set $S$.

For the vanishing conditions, we have the following. Note that we only consider half of the vanishing conditions, as in the formal setting it only makes sense to consider conditions on the leading few terms. Also, for convenience, we only consider the generic case. 
Proposition 8.26. Over the generic point $(E, x, c, q, t) \in \mathcal{E}^{4}$ and for integers $r, l$, consider the space of formal difference operators $D$ mapping $P_{0}(2 c ; q, t)$ to $P_{0}(2 c+l q ; q, t)$ such that $D \prod_{i} T_{i}^{r / 2}$ involves only integer shifts. If the left coefficients of both $D$ and

$$
\prod_{1 \leq i \leq n} \Gamma_{q}\left(x+r q / 2-z_{i}\right)^{-1} D \prod_{1 \leq i \leq n} \Gamma_{q}\left(x-z_{i}\right)
$$

are holomorphic along all hypersurfaces of the form $z_{i} \in x+r q / 2+k q, k \in \mathbb{Z}$, then the left coefficients of both $\hat{D}$ and

$$
\prod_{1 \leq i \leq n} \Gamma_{q}\left(x-c+(r-l) q / 2-z_{i}\right)^{-1} \hat{D} \prod_{1 \leq i \leq n} \Gamma_{q}\left(x-c-z_{i}\right)
$$

are holomorphic along all hyperplanes of the form $z_{i} \in x-c+(r-l) q / 2+k q, k \in \mathbb{Z}$.

Proof. By definition, we have

$$
\hat{D}=\mathcal{D}_{q, t}^{(n)}(c+l q / 2) D \mathcal{D}_{q, t}^{(n)}(-c) .
$$

There are only countably many hypersurfaces of the form $z_{i}=y$ on which some left coefficient of $\mathcal{D}_{q, t}^{(n)}(-c)$ and $\mathcal{D}_{q, t}^{(n)}(c+l q / 2)$ has a pole (including poles of the meromorphic sections of equivariant gerbes corresponding to the leading coefficients). Since $x$ is generic, it follows that all three factors on the right are holomorphic on the given orbits of hypersurfaces, and thus so is the product.

The claim for

$$
\prod_{1 \leq i \leq n} \Gamma_{q}\left(x-c+(r-l) q / 2-z_{i}\right)^{-1} \hat{D} \prod_{1 \leq i \leq n} \Gamma_{q}\left(x-c-z_{i}\right)
$$

analogously reduces to checking possible poles of

$$
\prod_{1 \leq i \leq n} \Gamma_{q}\left(x-z_{i}\right)^{-1} \mathcal{D}_{q, t}^{(n)}(-c) \prod_{1 \leq i \leq n} \Gamma_{q}\left(x-c-z_{i}\right)
$$

and

$$
\prod_{1 \leq i \leq n} \Gamma_{q}\left(x-c+(r-l) q / 2-z_{i}\right)^{-1} \mathcal{D}_{q, t}^{(n)}(c+l q / 2) \prod_{1 \leq i \leq n} \Gamma_{q}\left(x+r q / 2-z_{i}\right) .
$$

In each case, the gauging only multiplies the coefficients by holomorphic theta functions, so cannot introduce any new poles.

To get an analogue for the Selberg adjoint, there is a mild difficulty coming from the fact that the Selberg adjoint was only defined for $C_{n}$-symmetric operators, and the obvious extension does not make sense for formal operators. Luckily, the formal adjoint with respect to the inner product

$$
\int f\left(z_{1}, \ldots, z_{n}\right) g\left(-z_{1}, \ldots,-z_{n}\right) \prod_{1 \leq i<j \leq n} \frac{\Gamma_{q}\left(t \pm z_{i} \pm z_{j}\right)}{\Gamma_{q}\left( \pm z_{i} \pm z_{j}\right)} \prod_{1 \leq i \leq n} \frac{1}{\Gamma_{q}\left( \pm 2 z_{i}\right)} \mathrm{d} T
$$

does make sense for formal difference operators and formal gauging operators and agrees with the Selberg adjoint in the $C_{n}$-symmetric case. Using this as the definition of the Selberg adjoint for formal operators gives the following, which immediately implies consistency of the Fourier transform with the Selberg adjoint. 
Proposition 8.27. The operators $\mathcal{D}_{q, t}^{(n)}(c)$ are self-adjoint under the Selberg adjoint.

Proof. The Selberg adjoint has the correct leading term, so it suffices to show that

$$
\mathcal{D}_{q, t}^{(n)}(c)^{\operatorname{ad}_{t}} D_{q}^{(n)}(c \pm u ; t)=\prod_{1 \leq i \leq n} \vartheta\left(z_{i} \pm u\right) \mathcal{D}_{q, t}^{(n)}(c-q / 2)^{\operatorname{ad}_{t}} .
$$

Since $\prod_{1 \leq i \leq n} \vartheta\left(z_{i} \pm u\right)$ is self-adjoint, this reduces to checking that

$$
D_{q}^{(n)}(c \pm u ; t)^{\operatorname{ad}_{t}}=D_{q}^{(n)}(q / 2-c \pm u ; t),
$$

an easy verification.

Remark. In fact, one has in general

$$
D_{q}^{(n)}\left(u_{0}, \ldots, u_{2 d^{\prime}+1} ; t\right)^{\mathrm{ad}_{t}}=D_{q}^{(n)}\left(q / 2-u_{0}, \ldots, q / 2-u_{2 d^{\prime}+1} ; t\right),
$$

either by a direct computation or by using the fact that both are sections of the same Hom sheaf, and with the same leading coefficient.

We mention a couple of further consequences of the proof. First, the fact that the Fourier transform is determined by its values where we know it explicitly has consequences in the analytic setting. Indeed, in [27], a kernel function $\mathcal{K}_{c}^{(n)}(\vec{x} ; \vec{y} ; q, t)$ was constructed, with the property that for $D$ of degree $s, f$, or $s+f$, one had

$$
D_{\vec{x}} \mathcal{K}_{c}^{(n)}(\vec{x} ; \vec{y} ; q, t)=\hat{D}_{\vec{y}}^{\mathrm{ad}_{t}} \mathcal{K}_{c}^{(n)}(\vec{x} ; \vec{y} ; q, t) .
$$

It follows from the above proof and continuity that this holds for all operators which are global sections of the appropriate Hom spaces. In particular, this applies to operators of degree $2 s+2 f-$ $e_{1}-\cdots-e_{8}$ (i.e., the van Diejen/Komori-Hikami integrable system considered in Theorem 7.28), showing that the associated formal integral operator takes eigenvalue equations of this form to eigenvalue equations of the same form.

Also, we have already mentioned the consequence that the resulting deformations of $\operatorname{Sym}^{n}\left(\mathbb{P}^{2}\right)$ only depend (geometrically) on $E, q$, and $t$. It is worth mentioning the specific form that the given isomorphisms take. The isomorphism $\left.\left.\Gamma \mathcal{S}_{x_{0} ; q, t}^{(n)}\right|_{\mathbb{Z}(s+f)} \cong \Gamma \mathcal{S}_{x_{1} ; q, t}^{(n)}\right|_{\mathbb{Z}(s+f)}$ is given (up to a choice of element $\left.\left(x_{0}-x_{1}\right) / 3\right)$ by gauging by the operator

$$
\begin{aligned}
G_{d}\left(x_{0}, x_{1}\right):= & \prod_{1 \leq i \leq n} \Gamma_{q}\left(-\frac{(d-1) q}{2}-\frac{2 x_{0}+x_{1}}{3} \pm z_{i}\right) \mathcal{D}_{q, t}^{(n)}\left(\left(x_{0}-x_{1}\right) / 3\right) \\
& \times \prod_{1 \leq i \leq n} \Gamma_{q}\left(-\frac{(d-1) q}{2}-\frac{x_{0}+2 x_{1}}{3} \pm z_{i}\right)^{-1}
\end{aligned}
$$

in degree $d$; i.e., the action on morphisms from $d_{1}(s+f)$ to $d_{2}(s+f)$ is given by

$$
D \mapsto G_{d_{2}}\left(x_{0}, x_{1}\right) D G_{d_{1}}\left(x_{0}, x_{1}\right)^{-1} .
$$

In particular, we see that when $\left(x_{0}-x_{1}\right) / 3$ is 3 -torsion, the resulting automorphism is still quite nontrivial. In addition, the translation symmetry of the category involves changing $x_{0}$, and thus although one can identify it with a graded algebra at the cost of choosing an element $q / 3$, the resulting graded algebra does not actually have a representation in (finite) difference operators. If we restrict to the "anticanonical" model, i.e., to $\mathbb{Z}(3 s+3 f)$, then the Hom space of degree $3 s+3 f$ contains the 1-dimensional subspace of operators of degree $s$, spanned by

$$
G_{0}\left(x_{0}, x_{0}-3 q / 2\right)=\prod_{1 \leq i \leq n} \Gamma_{q}\left(-x_{0} \pm z_{i}\right) \mathcal{D}_{q, t}^{(n)}(-q / 2) \prod_{1 \leq i \leq n} \Gamma_{q}\left(-q / 2-x_{0} \pm z_{i}\right)^{-1} .
$$


If we adjoin the formal inverse of such an operator, then the result in degree 0 may be identified with a filtered algebra of formal difference operators. We can include elements of degree not a multiple of 3 at the cost of choosing $q / 3$ and allowing some $\Gamma_{q}$ factors and shifts by multiples of $q / 3$. Indeed, Proposition 8.15 tells us (assuming compatible choices when dividing by 3 ) that $G_{d}\left(x_{1}, x_{2}\right) G_{d}\left(x_{0}, x_{2}\right)=G_{d}\left(x_{0}, x_{2}\right)$, and thus the various isomorphisms between categories with parameter $x_{0}+k q / 2$ are all compatible. It follows that if we compose an operator mapping $d_{1}(s+f)$ to $d_{2}(s+f)$ with the formal operators giving isomorphisms $x_{0} \mapsto x_{0}+d_{1} q / 2$ and $x_{0}+d_{2} q / 2 \mapsto x_{0}$, then the result will be compatible with compositions and will be the same as if we only used the spaces with $d_{1}=0$. Of course, even in the univariate setting, the resulting algebra is not likely to be easy to describe in any direct fashion!

One final thing to mention is that our description of first-order operators in Lemma 8.8 as well as our description of the operators $\mathcal{D}_{q, t}^{(n)}(c)$ are both quite well suited to considering degenerations of the tuple $(E, c, q, t)$. In light of the fact that operators of degree $s+f$ are generically very ample, we can give at least indirect descriptions of the limiting algebras $\bigcup_{d} \mathcal{S}_{\eta^{\prime} ; q, t}^{(n)}(0, d(s+f))$ by specifying their elements of degree 1 , and understanding the extension to the whole category simply requires keeping track of the elements of degree $f$ as well.

Taking the limit can be somewhat tricky in general, as it may be necessary to gauge by suitable functions before the limit is well-defined. The simplest approach is to choose a suitable gauge transformation to make the operators elliptic before taking the limit; this introduces additional parameters which we can then eliminate by a further limit. Indeed, we find that for any operator $D \in \Gamma \mathcal{S}_{\eta^{\prime} ; q, t}^{(n)}\left(0, d s+d^{\prime} f\right)$, the gauge transformation

$$
\begin{aligned}
& \prod_{1 \leq i \leq n} \frac{\Gamma_{q}\left(\eta^{\prime}-d q / 2-d^{\prime} q+(n-1) t+v_{0}+v_{1}+v_{2} \pm z_{i}\right)}{\Gamma_{q}\left(-d q / 2+v_{0} \pm z_{i},-d q / 2+v_{1} \pm z_{i},-d q / 2+v_{2} \pm z_{i}\right)} \\
& \quad \times D \prod_{1 \leq i \leq n} \frac{\Gamma_{q}\left(v_{0} \pm z_{i}, v_{1} \pm z_{i}, v_{2} \pm z_{i}\right)}{\Gamma_{q}\left(\eta^{\prime}+(n-1) t+v_{0}+v_{1}+v_{2} \pm z_{i}\right)}
\end{aligned}
$$

is elliptic (for fixed $v_{0}, v_{1}, v_{2}$ ). We can then take the limit as $p \rightarrow 0$ and remove the parameters by gauging back by an appropriate product of $q$-Pochhammer symbols $(x ; q)_{\infty}:=\prod_{0 \leq j}\left(1-q^{j} x\right)$. We obtain a limit $\Gamma \mathcal{S}_{\eta^{\prime} ; q, t ; *}^{(n)}\left(0, s+d^{\prime} f\right)$ (with $q, t, \eta^{\prime}$ and $\vec{z}$ in the multiplicative group) consisting of operators of the form

$$
\sum_{\sigma \in\{ \pm 1\}^{n}} \prod_{1 \leq i \leq n} \frac{z_{i}^{\sigma_{i}} f\left(z_{i}^{\sigma_{i}}\right)}{1-z_{i}^{2 \sigma_{i}}} \prod_{1 \leq i<j \leq n} \frac{1-t z_{i}^{\sigma_{i}} z_{j}^{\sigma_{j}}}{1-z_{i}^{\sigma_{i}} z_{j}^{\sigma_{j}}} \prod_{1 \leq i \leq n} T_{i}^{\sigma_{i} / 2}
$$

where $f(z)$ is a univariate Laurent polynomial with exponents ranging from $1-d^{\prime}$ to $d^{\prime}-1$ satisfying the condition $\left[z^{d^{\prime}-1}\right] f(z)=q \eta^{\prime}\left[z^{1-d^{\prime}}\right] f(z)$ on its extreme coefficients. The Fourier transformation has a corresponding limit, represented by operators $\mathcal{D}_{q, t: *}^{(n)}(c)$ satisfying

$$
\begin{aligned}
\mathcal{D}_{q, t: *}^{(n)}(c) & \prod_{1 \leq i \leq n} \frac{\left((v c d) z_{i}^{ \pm 1} ; q\right)_{\infty}}{\left((v / c d) z_{i}^{ \pm 1} ; q\right)_{\infty}} \mathcal{D}_{q, t: *}^{(n)}(d) \\
= & \prod_{1 \leq i \leq n} \frac{\left((v d) z_{i}^{ \pm 1} ; q\right)_{\infty}}{\left((v / d) z_{i}^{ \pm 1} ; q\right)_{\infty}} \mathcal{D}_{q, t: *}^{(n)}(c d) \prod_{1 \leq i \leq n} \frac{\left((v c) z_{i}^{ \pm 1} ; q\right)_{\infty}}{\left((v / c) z_{i}^{ \pm 1} ; q\right)_{\infty}}
\end{aligned}
$$

with leading term

$$
\prod_{1 \leq i \leq n} \frac{\theta_{q^{1 / 2}}\left(-1 / z_{i}\right)}{\theta_{q^{1 / 2}}\left(-1 / c z_{i}\right)} \prod_{1 \leq i \leq j \leq n} \frac{\left(1 / c^{2} z_{i} z_{j} ; q\right)_{\infty}}{\left(1 / z_{i} z_{j} ; q\right)_{\infty}} \prod_{1 \leq i<j \leq n} \frac{\left(t / z_{i} z_{j} ; q\right)_{\infty}}{\left(t / c^{2} z_{i} z_{j} ; q\right)_{\infty}} T_{\omega}(c)
$$


and special case

$$
\mathcal{D}_{q, t: *}^{(n)}\left(q^{-1 / 2}\right)=\sum_{\sigma \in\{ \pm 1\}^{n}} \prod_{1 \leq i \leq n} \frac{z_{i}^{\sigma_{i}}}{1-z_{i}^{2 \sigma_{i}}} \prod_{1 \leq i<j \leq n} \frac{1-t z_{i}^{\sigma_{i}} z_{j}^{\sigma_{j}}}{1-z_{i}^{\sigma_{i}} z_{j}^{\sigma_{j}}} \prod_{1 \leq i \leq n} T_{i}^{\sigma_{i} / 2} .
$$

Note that taking $v=0$ in $(8.1)$ gives $\mathcal{D}_{q, t: *}^{(n)}(c) \mathcal{D}_{q, t: *}^{(n)}(d)=\mathcal{D}_{q, t: *}^{(n)}(c d)$, so that we may interpret $\mathcal{D}_{q, t: *}^{(n)}(c)$ as a fractional power of the operator for $c=q^{-1 / 2}$, which in turn is a lowering operator appearing in the theory of Koornwinder polynomials. We can further extend this limit to the case of blowups in sufficiently general position (i.e., with all $x_{i}$ finite) by imposing the appropriate conditions on the leading coefficients; this is how we tested the $n=r=2$ case of Conjecture 7.30.

Another noteworthy limit involves gauging by a translation so as to break the $z \mapsto 1 / z$ symmetry, and taking a limit in the resulting parameter. This gives a Fourier transform represented by operators satisfying

$$
\mathcal{D}_{q, t: * *}^{(n)}(c) \prod_{1 \leq i \leq n} \frac{\left((v c d) z_{i} ; q\right)_{\infty}}{\left((v / c d) z_{i} ; q\right)_{\infty}} \mathcal{D}_{q, t: * *}^{(n)}(d)=\prod_{1 \leq i \leq n} \frac{\left((v d) z_{i} ; q\right)_{\infty}}{\left((v / d) z_{i} ; q\right)_{\infty}} \mathcal{D}_{q, t: * *}^{(n)}(c d) \prod_{1 \leq i \leq n} \frac{\left((v c) z_{i} ; q\right)_{\infty}}{\left((v / c) z_{i} ; q\right)_{\infty}}
$$

with leading term

$$
\prod_{1 \leq i \leq n} \frac{\theta_{q^{1 / 2}}\left(-1 / z_{i}\right)}{\theta_{q^{1 / 2}}\left(-1 / c z_{i}\right)} T_{\omega}(c)
$$

and special case

$$
\mathcal{D}_{q, t: * *}^{(n)}\left(q^{-1 / 2}\right)=\sum_{I \subset\{1, \ldots, n\}}(-1)^{|I|} t^{|I|(|I|-1) / 2} \prod_{1 \leq i \leq n} z_{i}^{-1} \prod_{i \in I, j \notin I} \frac{z_{j}-t z_{i}}{z_{j}-z_{i}} \prod_{i \in I} T_{i}^{1 / 2} \prod_{i \notin I} T_{i}^{-1 / 2},
$$

a.k.a. the lowering operator for $\mathrm{GL}_{n}$-type Macdonald polynomials. The Hom spaces of degree $s+d^{\prime} f$ have similar, if somewhat more complicated forms, obtained by gauging the $q^{-1 / 2}$ case of the Fourier transform operator by suitable products of Pochhammer symbols. We omit the details, except to note that the results again look like operators arising in Macdonald theory.

There are some other symmetry breaking limits (e.g., the image of $\eta^{\prime} \rightarrow 0$ under the Fourier transform); we omit the details. Of course, such symmetry-breaking limits have unfortunate effects on the Bruhat ordering; for instance, the "leading term" must now incorporate all $\sim 2^{n}$ $S_{n}$-orbits corresponding to the given $C_{n}$-orbit of weights. As a result, in more degenerate cases, it can be difficult to figure out the correct way to compactify the algebra. This can be fixed in some cases by realizing that the $S_{n}$-symmetric operator is actually a shadow of a $C_{n}$-symmetric operator acting on a power of a reducible curve (a hyperelliptic curve of arithmetic genus 1). Similarly, there are differential limits living on a power of the nonreduced curve $y^{2}=0$.

\section{Deformations of Hilbert schemes}

One potential issue with studying the category of sheaves on $\mathcal{S}$, even if one can resolve the uncertainty in the definition, is that the commutative projective scheme it deforms, the symmetric power of a surface, is singular. This suggests that one should look for an analogous deformation in which the symmetric power is replaced by its natural resolution, namely the Hilbert scheme of points.

The Picard group of the $n$-point Hilbert scheme of $X$ is still discrete (and isomorphic to the Néron-Severi group), with $\operatorname{Pic}\left(\operatorname{Hilb}^{n}(X)\right) \cong \operatorname{Pic}(X) \oplus \mathbb{Z}$ for $n>1$. The copy of $\operatorname{Pic}(X)$ in $\operatorname{Pic}\left(\operatorname{Hilb}^{n}(X)\right)$ is the pullback of $\operatorname{Pic}\left(\operatorname{Sym}^{n}(X)\right) \cong \operatorname{Pic}(X)$, and since the map $\operatorname{Hilb}^{n}(X) \rightarrow$ 
$\operatorname{Sym}^{n}(X)$ is a birational morphism, the global sections of any such bundle will be the same on either $2 n$-fold. Thus if we deform the category of line bundles on $\operatorname{Hilb}^{n}(X)$, the subcategory corresponding to $\operatorname{Sym}^{n}(X)$ should be precisely $\mathcal{S}$. As we saw for the symmetric power, twisting by line bundles cannot be expected to give an endofunctor, and thus we expect that twisting by the additional generator of $\operatorname{Pic}\left(\operatorname{Hilb}^{n}(X)\right)$ should also change the parameters.

There is, of course, only one remaining parameter we could reasonably shift, and thus we should consider what happens as we change $t$. Consider for the moment the $\mathbb{P}^{1} \times \mathbb{P}^{1}$ case. The sections of $\mathcal{S}$ are (for generic $t$ ) cut out by the condition that both $\mathcal{D}$ and

$$
\prod_{1 \leq i<j \leq n} \Gamma_{q}\left(t \pm z_{i} \pm z_{j}\right) \mathcal{D} \prod_{1 \leq i<j \leq n} \Gamma_{q}\left(t \pm z_{i} \pm z_{j}\right)^{-1}
$$

are sections of the corresponding parameter-free category. Incorporating a shift in $t$ here is then mostly straightforward: we should be considering operators such that both $\mathcal{D}$ and

$$
\prod_{1 \leq i<j \leq n} \Gamma_{q}\left(t+a_{2} q \pm z_{i} \pm z_{j}\right) \mathcal{D} \prod_{1 \leq i<j \leq n} \Gamma_{q}\left(t+a_{1} q \pm z_{i} \pm z_{j}\right)^{-1}
$$

are holomorphic for some integers $a_{1}$ and $a_{2}$. We should also note the effect on twisting, which boils down to noting that shifting $t$ acts on the standard polarization as $P_{d}\left(\eta^{\prime} ; q, t+a q\right)=$ $P_{d-(n-1) a}\left(\eta^{\prime} ; q, t\right)$.

With this in mind (and incorporating the conditions corresponding to $x_{1}, \ldots, x_{m}$ ), define a product of $\Gamma_{q}$ symbols for any element of $\operatorname{Pic}\left(\operatorname{Hilb}^{n}(X)\right)=\mathbb{Z}\left\langle\delta, s, f, e_{1}, \ldots, e_{m}\right\rangle$ as follows:

$$
\begin{aligned}
& \Delta_{x_{1}, \ldots, x_{m} ; q, t}\left(a \delta+d s+d^{\prime} f-r_{1} e_{1}-\cdots-r_{m} e_{m} ; \vec{z}\right) \\
& \quad=\prod_{1 \leq i<j \leq n} \Gamma_{q}\left(t+a q \pm z_{i} \pm z_{j}\right) \prod_{\substack{1 \leq i \leq n \\
1 \leq j \leq m}} \Gamma_{q}\left(\left(r_{j}+(1-d) / 2\right) q-x_{j} \pm z_{i}\right) .
\end{aligned}
$$

Also, let $\pi: \operatorname{Pic}\left(\operatorname{Hilb}^{n}(X)\right) \rightarrow \mathbb{Z}\langle s, f\rangle$ be given by $\pi(s)=s, \pi(f)=f, \pi\left(e_{i}\right)=0$ and $\pi(\delta)=$ $-(n-1) f$. Then (for $n>1) \mathcal{S}_{\eta^{\prime}, x_{1}, \ldots, x_{m} ; q, t}^{(n)}(v, w)$ is defined to be the subsheaf of $\mathcal{S}_{\eta^{\prime} ; q}^{(n)}(\pi(v), \pi(w))$ (the spherical algebra of the construction corresponding to the master DAHA) consisting for generic parameters of the operators $\mathcal{D}$ such that $\Delta_{x_{1}, \ldots, x_{m} ; q, t}(w, \vec{z}) \mathcal{D} \Delta_{x_{1}, \ldots, x_{m} ; q, t}(v, \vec{z})^{-1}$ is also a local section of the correspondingly twisted master spherical algebra. It is easy to see that when $v$ and $w$ have the same coefficient of $\delta$, this imposes the same vanishing conditions as the original definition of $\mathcal{S}$, and thus this indeed extends the category to the larger group of objects. The elementary transformation symmetries automatically extend (using the obvious definition for $\left.\mathcal{S}^{\prime(n)}\right)$, as does the $t \mapsto q-t$ symmetry, which now acts nontrivially on the objects (negating $\delta$ ); for the Fourier transform, see below.

We should note in passing that this is closely related to a construction valid for general affine Weyl groups. We have already mentioned that translating part of the system of parameters of such a DAHA or spherical algebra by $q$ gives a Morita equivalent algebra, and thus in particular a natural bimodule. More generally, we may translate any point of any $T_{i}$ by any multiple of $q$ without affecting generic Morita equivalence, and the associated bimodule can be constructed as the tensor product of the bimodules for the atomic shifts. As a result, we may construct a sheaf category in which the objects are the lattice of different ways we may shift the parameters (i.e., with rank equal to the total number of components of the independent $T_{i}$ ), and each Hom bimodule is the relevant Morita equivalence. Each of the original bimodules embeds in $\mathcal{H}_{\widetilde{W}, W ; \gamma}(X)$ (or $\mathcal{H}_{\widetilde{W} ; \gamma}(X)$ in the DAHA version) as operators satisfying appropriate vanishing conditions and thus the construction has a natural extension to general parameters. (Note that this construction only allows us to shift those parameters which are associated to roots of $W$ itself, so to obtain the $C^{\vee} C_{n}$ version in this way we need to associate the $\vec{x}$ parameters to $s_{n}$ 
rather than $s_{0}$.) This may be thought of as a generalization (to the elliptic level and arbitrary numbers of parameters) of the construction of [12].

As in the symmetric power case, we would like to show that the above generic conditions extend to give a strongly flat family of categories (i.e., not only flat but such that each fiber injects in the algebra of meromorphic difference operators). The first step of the construction carries over: replacing the $t$-dependent factor of $\Delta$ by the appropriate nonsymmetric version gives us a natural corresponding extension of $\mathcal{H}$. Unfortunately, the argument of Theorem 7.12 fails in this case to show flatness of the extended $\mathcal{H}$. The key difference here is that the roots involved in the conditions corresponding to $x_{1}, \ldots, x_{m}$ form a root system of type $A_{1}^{n}$. If we make the analogous construction for any other root system, then we will find that there are some leading terms for which the only vanishing condition involves a non-simple root, and such that any of the terms covered by that leading term do not have the corresponding vanishing condition. In particular, since the $t$-dependent vanishing conditions live on a root system of type $D_{n}$, this argument fails, with the notable exception of $n=2$.

Proposition 9.1. The sheaf category $\mathcal{S}_{\eta^{\prime}, x_{1}, \ldots, x_{m} ; q, t}^{(2)}$ defined above is locally free, and the map from any Hom bimodule to the sheaf bimodule of meromorphic operators is injective on fibers.

Proof. We argue essentially as in the symmetric power case. We first note that by using elementary transformations and the analogue for $t$, that we may arrange for the vanishing condition on the identity to be trivial. Then for any $w$ in the appropriate Bruhat interval, if there is any nontrivial vanishing condition associated to $t$ or $x_{i}$ in the interval, then there is such a condition at $w$ and associated to a simple reflection that makes $w$ shorter. Thus we may obtain the restriction of the given Hom sheaf to $[\leq w]$ by composing the appropriate rank 1 Hecke algebra (using only those parameter still active) to the restriction to $[\leq s w]$.

Remark. For each simple root of $W$ and each associated parameter $t$ or $x_{i}$, the existence of a nontrivial vanishing condition of $c_{w}$ along $t+\alpha$ or $x_{i}+\alpha$ is equivalent to the alcove associated to $w$ being on the appropriate side of a hyperplane orthogonal to $\alpha$. For $n=2$, there is thus a square (itself a Bruhat order ideal) such that any alcove in the square has no vanishing condition at $t$ (so we may ignore the parameter) and similarly for each $x_{i}$. For $n>2$, there is a corresponding shape in which no simple root (or its negative) has a vanishing condition for $t$; if one could prove strong flatness for the corresponding Bruhat order ideal, this would imply strong flatness in general.

There is, however, one more special case in which we can prove strong flatness. This has to do with the additional symmetry of the spherical algebra in the DAHA case. In particular, we noted that both the algebra for $t$ and the algebra for $t+q$ could be obtained from the Hecke algebra via an analogue of the construction of the spherical algebra, and that moreover there were analogous constructions of intertwining bimodules. But these intertwiners are precisely the Hom sheaves we want.

Proposition 9.2. Suppose the coefficient of $\delta$ in $v$ is -1 , 0 , or 1 . Then $\mathcal{S}_{\eta^{\prime}, x_{1}, \ldots, x_{m} ; q, t}^{(n)}(0, v)$ is locally free and injects on fibers into the sheaf bimodules of meromorphic operators.

Remark. Actually, there are two more cases in which we can prove strong flatness. If $a \geq d$, then the $t$-dependent vanishing conditions become vacuous, and thus the claim reduces to strong flatness in the version without a $t$ parameter, where the usual argument works. By the $t \mapsto q-t$ symmetry, this implies strong flatness when $a \leq-d$.

Not only is this further evidence that this construction is well-behaved, but this also has several useful consequences. The most significant of these is that the construction of the intertwining 
bimodules allows us to understand the case $t=0$. In particular, let $v \in \mathbb{Z}\left\langle s, f, e_{1}, \ldots, e_{m}\right\rangle$, and consider the Hom sheaf

$$
\mathcal{S}_{\eta^{\prime}, x_{1}, \ldots, x_{m} ; q, 0}^{(n)}(0, \delta+v) .
$$

By the infinite analogue of Proposition 4.64, these operators are all of the form $\sum_{w \in C_{n}} w \mathcal{D}$, where $\mathcal{D}$ is a section of the left twist by $\mathcal{O}_{X}\left(D_{w_{C_{n}}}\right)$ of the corresponding spherical module. Since $t=0$, the spherical module is just the tensor product of $n$ copies of the univariate spherical module $M_{\eta^{\prime}, x_{1}, \ldots, x_{m} ; q}^{(1)}(v)$, and thus the Hom sheaf is spanned by elements of the form

$$
\left(\sum_{w \in C_{n}} w\right) \frac{1}{\prod_{1 \leq i \leq n} \vartheta\left(2 z_{i}\right) \prod_{1 \leq i<j \leq n} \vartheta\left(z_{i} \pm z_{j}\right)} D_{1}\left(z_{1}\right) \cdots D_{n}\left(z_{n}\right)
$$

with each $D_{i}$ in the univariate spherical module. Summing over the normal subgroup of order $2^{n}$ turns each $D_{i}$ into a general element of the corresponding spherical algebra, giving

$$
\sum_{\pi \in S_{n}} \pi \frac{1}{\prod_{1 \leq i<j \leq n} \vartheta\left(z_{i} \pm z_{j}\right)} D_{1}^{\prime}\left(z_{1}\right) \cdots D_{n}^{\prime}\left(z_{n}\right)=\frac{1}{\prod_{1 \leq i<j \leq n} \vartheta\left(z_{i} \pm z_{j}\right)} \operatorname{det}_{1 \leq i, j \leq n} D_{i}^{\prime}\left(z_{j}\right)
$$

with each $D_{i}^{\prime}$ in the univariate spherical algebra. (Note that since these are univariate operators in distinct variables, the determinant makes sense.) We thus conclude the following.

Proposition 9.3. For any $v \in\left\langle s, f, e_{1}, \ldots, e_{m}\right\rangle$, there is a natural isomorphism of sheaf bimodules

$$
\mathcal{S}_{\eta^{\prime}, x_{1}, \ldots, x_{m} ; q, 0}^{(n)}(0, \delta+v) \cong \wedge^{n} \mathcal{S}_{\eta^{\prime}, x_{1}, \ldots, x_{m} ; q, 0}^{(1)}(0, v) .
$$

If $q=0$ and $v$ corresponds to an acyclic line bundle on the corresponding rational projective surface $X_{m}$, then the exterior power is also acyclic and we deduce

$$
\Gamma \mathcal{S}_{\eta^{\prime}, x_{1}, \ldots, x_{m} ; 0,0}^{(n)}(0, \delta+v) \cong \wedge^{n} \Gamma\left(X_{m} ; \mathcal{O}_{X_{m}}(v)\right) .
$$

Any element of either side (which differ only in the product of theta functions corresponding to the $D_{n}$ roots) gives a rational function on $X_{m}^{n}$, and the description as an exterior power tells us that the corresponding map to projective space is in fact a map to a Grassmannian: each point in $X_{m}$ determines a linear functional on $\Gamma\left(X_{m} ; \mathcal{O}_{X_{m}}(v)\right)$, and the map takes $X_{m}^{n}$ to the $n$-dimensional span of the corresponding linear functionals.

This is clearly invariant under permutations of the $n$-tuple, so gives a rational map on the symmetric power, and thus on the Hilbert scheme. Since we may also view this as the Grassmannian of $\left(h^{0}\left(\mathcal{O}_{X_{m}}(v)\right)-n\right)$-dimensional subspaces of $\Gamma\left(X_{m} ; \mathcal{O}_{X_{m}}(v)\right)$, we see that the map on the Hilbert scheme takes a given ideal sheaf $I$ to the subspace $\Gamma\left(X_{m} ; I(v)\right)$, assuming this has the correct dimension. In particular, for any sufficiently ample $v, I(v)$ will always be acyclic and globally generated, and thus we obtain an embedding of the Hilbert scheme in the Grassmannian. From known facts about acyclicity and Hilbert polynomials of line bundles on the Hilbert scheme [9], the Plücker coordinates in fact form all global sections of the given line bundle on the Hilbert scheme. (This line bundle has the form $-\Delta / 2+v$, where $\Delta$ is the discriminant, i.e., the divisor where the corresponding $n$-point subscheme is nonreduced.)

We thus conclude that for $q=t=0$ and $v$ sufficiently (relatively) ample on $X_{m}$, the (left) vector bundle $\mathcal{S}_{\eta^{\prime}, x_{1}, \ldots, x_{m} ; 0,0}^{(n)}(0, \delta+v)$ on $\mathbb{P}^{n}$ may be identified with the direct image of $\mathcal{O}_{X_{m}}(v)$ under the map $\operatorname{Hilb}^{n}\left(X_{m}\right) \rightarrow \operatorname{Sym}^{n}\left(\mathbb{P}^{1}\right) \cong \mathbb{P}^{n}$. Moreover, since we obtained this identification by using the actual values of the "operators" in $\mathcal{S}$, these elements satisfy precisely the same relations as their counterparts on the Hilbert scheme. 
Note that although this indicates that there is a strong relation between our construction and the Hilbert scheme, it is not quite enough to tell us that $\mathcal{S}$ is a true deformation of the Hilbert scheme: the problem is that there could conceivably be additional global sections involving larger multiples of $\delta$. For $n=2$, we can resolve this issue: it is straightforward (if tedious) to use the Bruhat filtration to compute the Euler characteristics of Hom sheaves of $\mathcal{S}$, and we find in particular that when $a(-\Delta / 2)+v$ is acyclic over $\mathbb{P}^{n}$ on the Hilbert scheme, the Euler characteristic of the Hom sheaf agrees with the Euler characteristic of the corresponding line bundle on the Hilbert scheme. Since we've shown an isomorphism for a large class of very ample divisors, it follows that there is a cone in which the direct image of the line bundle on the Hilbert scheme injects in the corresponding Hom sheaf, and must therefore be isomorphic. We thus conclude that (in a somewhat vague sense) $\mathcal{S}^{(2)}$ is indeed the desired deformation of the Hilbert scheme. (Note that this argument does not apply to the obvious category associated to $\operatorname{Hilb}^{2}\left(\mathbb{P}^{2}\right)$ in the absence of additional acyclicity results.)

Unfortunately, the calculation of Euler characteristics of Hom sheaves is not only tedious but subject to combinatorial explosion: the contribution from each subquotient depends in a nontrivial and apparently nonuniform way on the corresponding parabolic subgroup, and thus the general computation requires computing a subsum for each parabolic subgroup, a total of $2^{n}$ individual sums. Moreover, the condition under which the divisor on the Hilbert scheme is acyclic involves an upper bound on $a$ depending on $d$, $d^{\prime}$, etc., and that bound is not preserved if we subtract $2 s+2 f-e_{1}-\cdots-e_{m}$; as a result, we cannot expect to simplify the calculation by using an induction on $d$ and $n$. A further issue arises in the final step of comparing to the Hilbert scheme: the formula of [9] is not quite explicit; it depends on a certain universal power series which is only known in low degree, and thus the Euler characteristics are only known for small $n$.

Still, it seems reasonable on the above evidence to conjecture that $\mathcal{S}$ indeed provides a flat deformation of $\operatorname{Hilb}^{n}\left(X_{m}\right)$ for every $n$.

Assuming that this is true, we would like to know that the deformation depends only on the original rational surface rather than on the way in which we obtained it as a blowup. As in the symmetric power case, elementary transformations are easy to deal with, and it is only the analogue of the Fourier transform that we must consider. There is a clear guess as to how the Fourier transformation should be defined, at least when $q$ is not torsion: use the same operators $\mathcal{D}_{q, t}(c)$ and simply adjust $t$ as appropriate. This leads to an obvious question, namely whether this extension of the Fourier transform is still well-defined when $q$ is torsion. As in the symmetric power case, we can first ask for this to hold for formal difference operators, where it takes the form

$$
D \mapsto \mathcal{D}_{q, t+a q}^{(n)}(c+b q / 2) D \mathcal{D}_{q, t}^{(n)}(-c)
$$

for $a, b \in \mathbb{Z}$. Since we know the usual Fourier transform

$$
\hat{D}=\mathcal{D}_{q, t}^{(n)}(c+(b+(n-1) a) q / 2) D \mathcal{D}_{q, t}^{(n)}(-c)
$$

is well-defined, we may factor this as

$$
\mathcal{D}_{q, t+a q}^{(n)}(c+b q / 2) \mathcal{D}_{q, t}^{(n)}(-c-(b+(n-1) a) q / 2) \hat{D},
$$

and thus reduce to showing that the formal operator

$$
\mathcal{D}_{q, t+a q}^{(n)}(c+b q / 2) \mathcal{D}_{q, t}^{(n)}(-c-(b-(n-1) a) q / 2)
$$

remains holomorphic when $q$ is torsion. This then easily reduces to the analogous statement for

$$
\mathcal{D}_{q, t+q}^{(n)}(-c-(n-1) q / 2) \mathcal{D}_{q, t}^{(n)}(c) .
$$


Indeed, for $a>0$, we can factor the operator in question into $a$ operators of the given form, while for $a<0$ we have a similar factorization of the inverse, and the leading term is clearly nonzero.

Proposition 9.4. The formal operator

$$
\mathcal{D}_{q, t+q}^{(n)}(-c-(n-1) q / 2) \mathcal{D}_{q, t}^{(n)}(c)
$$

is a global section of $\mathcal{S}_{2 c ; q, t}^{(n)}(0, \delta+(n-1) s)$.

Proof. The Hom sheaf $\mathcal{S}_{2 c ; q, t}^{(n)}(0, \delta+(n-1) s)$ is a vector bundle, and the description as an alternating power for $t=0$ tells us that it has Euler characteristic 1 and is acyclic away from a finite set of hypersurfaces, not containing any component on which $q$ is torsion. Similarly, $\mathcal{S}_{2 c ; q, t}^{(n)}(-s, \delta+(n-1) s)$ has Euler characteristic $n+1$ and is also acyclic on a suitable open subset. Moreover, a section of either vector bundle with vanishing leading coefficient is 0; by semicontinuity (and flatness for Bruhat intervals), it suffices to check this for $t=0$, where it again reduces to facts about the univariate case. Let $D(c)$ be any nonzero local section of $\mathcal{S}_{2 c ; q, t}^{(n)}(0, \delta+(n-1) s)$. Then as $u$ varies,

$$
D(c) D_{q}^{(n)}(c \pm u ; t)
$$

spans an $n+1$-dimensional family of sections of $\mathcal{S}_{2 c ; q, t}^{(n)}(-s, \delta+(n-1) s)$, which must therefore be everything. Applying the same argument on the left and comparing the dependence of the leading coefficient on $u$, we conclude that

$$
D_{q}^{(n)}((n-1) q / 2+c \pm u ; t+q)^{-1} D(c) D_{q}^{(n)}(c \pm u ; t)
$$

is a section of $\mathcal{S}_{2 c ; q, t}^{(n)}(-s, \delta+(n-2) s)=\mathcal{S}_{2 c-q ; q, t}^{(n)}(0, \delta+(n-1) s)$ and thus

$$
D_{q}^{(n)}((n-1) q / 2+c \pm u ; t+q)^{-1} D(c) D_{q}^{(n)}(c \pm u ; t) \propto D(c-q / 2),
$$

with coefficient independent of $z_{i}$ and $u$.

Now, $D(c)$ has leading term

$$
F(\vec{z} ; c ; q, t) \prod_{1 \leq i \leq j \leq n} \frac{1}{\vartheta\left(-z_{i}-z_{j} ; q\right)_{n-1}} \prod_{1 \leq i<j \leq n} \vartheta\left(q+t-z_{i}-z_{j} ; q\right)_{n-2} T_{\omega}(-(n-1) q / 2),
$$

with $F(\vec{z} ; c ; q, t)$ holomorphic and $S_{n}$-invariant. The relation between $D(c)$ and $D(c-q / 2)$ gives a weak recurrence for the leading coefficient, namely that

$$
F\left(z_{1}-q / 2, \ldots, z_{n}-q / 2 ; c-q / 2 ; q, t\right) F\left(z_{1}, \ldots, z_{n} ; c ; q, t\right)^{-1}
$$

is independent of $\vec{z}$, and thus $F$ factors as

$$
F(\vec{z} ; c ; q, t)=G(c-\vec{z} ; q, t) H(c ; q, t)
$$

where we may as well absorb any $c$-dependence of $H$ into $D(c ; q, t)$ and thus make the recurrence exact.

If we specialize $c=-(n-1) q / 2$, then we still find that $\mathcal{S}_{-(n-1) q ; q, t}^{(n)}(0, \delta+(n-1) s)$ is generically 1 -dimensional by reference to the $t=0$ case. Since the operator $D_{q, t}^{(n)}(n-1)$ satisfies all of the 
requisite vanishing conditions, we conclude that $D(-(n-1) q / 2)$ is proportional to $D_{q, t}^{(n)}(n-1)$, and thus that

$$
G(-(n-1) q / 2-\vec{z} ; q, t) \propto \prod_{1 \leq i<j \leq n} \vartheta\left(t-z_{i}-z_{j}\right)
$$

so that (after rescaling) $D(c)$ has leading term

$$
\begin{gathered}
\prod_{1 \leq i \leq j \leq n} \frac{1}{\vartheta\left(-z_{i}-z_{j} ; q\right)_{n-1}} \prod_{1 \leq i<j \leq n} \vartheta\left(q+t-z_{i}-z_{j} ; q\right)_{n-2} \vartheta\left(2 c+t+(n-1) q-z_{i}-z_{j}\right) \\
\times T_{\omega}(-(n-1) q / 2) .
\end{gathered}
$$

We then find that

$$
\mathcal{D}_{q, t+q}^{(n)}(c+(n-1) q / 2) D(c)
$$

has the same leading term and satisfies the same defining recurrence as $\mathcal{D}_{q, t}^{(n)}(c)$, and thus by the proof of Proposition 8.13,

$$
D(c)=\mathcal{D}_{q, t+q}^{(n)}(-c-(n-1) q / 2) \mathcal{D}_{q, t}^{(n)}(c),
$$

from which the desired claim immediately follows.

Remark. When $n=2$, this is a first-order operator, and thus one can easily deduce an explicit formula from the leading coefficient. This turns out to be an operator we have already seen, albeit with a rather odd change of parameters: $\mathcal{D}_{q, t+q}^{(2)}(-c-q / 2) \mathcal{D}_{q, t}^{(2)}(c)=D_{q, t+q+2 c}^{(2)}(1)$, a special case of the curious identity $\mathcal{D}_{q, 2 u_{1}}^{(2)}\left(u_{2}-u_{3}\right) \mathcal{D}_{q, 2 u_{2}}^{(2)}\left(u_{3}-u_{1}\right) \mathcal{D}_{q, 2 u_{3}}^{(2)}\left(u_{1}-u_{2}\right)=1$. The latter can be proved by Zariski closure from the case $u_{2}=k q / 2+u_{3}$, which in turn follows by induction in $k$ from the known special case.

The operator considered in the proposition is the Fourier transform of the global section

$$
1 \in \Gamma \mathcal{S}_{-2 c ; q, t}^{(n)}(0, \delta+(n-1) f)
$$

As mentioned above, the usual $t \mapsto q-t$ symmetry extends to a symmetry that negates $\delta$, and thus we also find that the Fourier transform of the global section

$$
\prod_{1 \leq i<j \leq n} \vartheta\left(t \pm z_{i} \pm z_{j}\right) \in \Gamma \mathcal{S}_{-2 c ; q, t}^{(n)}(0,-\delta+(n-1) f)
$$

is a section of $\Gamma \mathcal{S}_{2 c ; q, t}^{(n)}(0,-\delta+(n-1) s)$. An easy induction then shows that

$$
\mathcal{D}_{q, t}^{(n)}(-c-a(n-1) q / 2) \prod_{1 \leq i<j \leq n} \vartheta\left(t \pm z_{i} \pm z_{j} ; q\right)_{a} \mathcal{D}_{q, t+a q}^{(n)}(c)
$$

is a section of $\mathcal{S}_{2 c ; q, t}^{(n)}(a \delta, a(n-1) s)$ for $a \geq 0$. For purposes of the following proof, denote this operator by $S_{-}^{(n)}(a ; c ; q, t)$.

Proposition 9.5. The Fourier transform extends to the Hilbert scheme category. 
Proof. As in the symmetric power case, this reduces to showing that the Fourier transform takes $\Gamma \mathcal{S}_{2 c ; q, t}^{(n)}\left(0, a \delta+d s+d^{\prime} f\right)$ to $\Gamma \mathcal{S}_{-2 c ; q, t}^{(n)}\left(0, a \delta+d^{\prime} s+d f\right)$. By the $t \mapsto q-t$ symmetry, it suffices to show this for $a \geq 0$. Let $D$ be a local section of this category on some open subset of parameter space. Then

$$
\begin{gathered}
\prod_{1 \leq i<j \leq n} \vartheta\left(t \pm z_{i} \pm z_{j} ; q\right)_{a} D \in \Gamma \mathcal{S}_{2 c ; q, t}^{(n)}\left(0, d s+\left(d^{\prime}+a(n-1)\right) f\right), \\
S_{-}^{(n)}\left(a ; c-\left(d^{\prime}-d\right) q / 2 ; q, t\right) D \in \Gamma \mathcal{S}_{2 c ; q, t}^{(n)}\left(0,(d+a(n-1)) s+d^{\prime} f\right),
\end{gathered}
$$

since each prefactor is itself a section of $\Gamma \mathcal{S}^{(n)}$. Both of these operators have well-behaved Fourier transforms, and we thus conclude that

$$
\begin{aligned}
& S_{-}^{(n)}\left(a ;-c+\left(d^{\prime}-d\right) q / 2 ; q, t\right) \hat{D} \in \Gamma \mathcal{S}_{2 c ; q, t}^{(n)}\left(0,\left(d^{\prime}+a(n-1)\right) s+d f\right) \\
& \prod_{1 \leq i<j \leq n} \vartheta\left(t \pm z_{i} \pm z_{j} ; q\right)_{a} \hat{D} \in \Gamma \mathcal{S}_{2 c ; q, t}^{(n)}\left(0, d^{\prime} s+(d+a(n-1)) f\right) .
\end{aligned}
$$

We claim that this implies $\hat{D} \in \Gamma \mathcal{S}_{2 c ; q, t}^{(n)}\left(0, a \delta+d^{\prime} s+d f\right)$ as required.

It suffices to show this on an open subset, and thus we may assume that $c$ and $t$ are in general position. The second claim shows that

$$
\begin{aligned}
\prod_{1 \leq i<j \leq n} & \Gamma_{q}\left(a q+t \pm z_{i} \pm z_{j}\right) \hat{D} \prod_{1 \leq i<j \leq n} \Gamma_{q}\left(t \pm z_{i} \pm z_{j}\right)^{-1} \\
& =\prod_{1 \leq i<j \leq n} \Gamma_{q}\left(t \pm z_{i} \pm z_{j}\right) \prod_{1 \leq i<j \leq n} \vartheta\left(t \pm z_{i} \pm z_{j} ; q\right)_{a} \hat{D} \prod_{1 \leq i<j \leq n} \Gamma_{q}\left(t \pm z_{i} \pm z_{j}\right)^{-1}
\end{aligned}
$$

is a section of the $t$-free version of the category, and thus it remains only to show that $\hat{D}$ itself is such a section. In other words, we need to show that dividing by $\vartheta\left(t \pm z_{i} \pm z_{j} ; q\right)_{a}$ does not introduce any poles. Now, the operator $S_{-}^{(n)}\left(a ;-c+\left(d^{\prime}-d\right) q / 2 ; q, t\right)$ is holomorphic away from the reflection hypersurfaces, and has leading (left) coefficient

$$
\frac{\prod_{1 \leq i<j \leq n} \vartheta\left(t-z_{i}-z_{j} ; q\right)_{a n} \vartheta\left(t \pm\left(z_{i}-z_{j}\right) ; q\right)_{a} \vartheta\left(2 c+t+z_{i}+z_{j}-\left(d^{\prime}-d+a(n-1)\right) q ; q\right)_{a}}{\prod_{1 \leq i \leq j \leq n} \vartheta\left(-z_{i}-z_{j} ; q\right)_{a(n-1)}} .
$$

If we take its inverse as a formal operator, the only poles that can arise are translates of those originally present and translates of the divisors on which the leading coefficient vanishes. We find in particular that the inverse remains (since $t$ and $c$ are generic) holomorphic on any divisor of the form $t+z_{i}+z_{j}=k q$. Since $S_{-}^{(n)}\left(a ;-c+\left(d^{\prime}-d\right) q / 2 ; q, t\right) \hat{D}$ is holomorphic away from the reflection hypersurfaces, it follows that $\hat{D}$ itself is holomorphic on the divisors $t+z_{i}+z_{j}=k q$, and thus by symmetry on any divisor $t \pm z_{i} \pm z_{j}=k q$. But this is precisely what we needed to show.

Remark. As in the symmetric power case, this tells us that the subcategory corresponding to $\mathbb{P}^{2}$ is indeed (up to fppf local isomorphism) independent of $c$.

When $q=0$, the category is equal to its center (taking the natural extension of the definition used in the symmetric power case), and thus we expect to obtain a family of commutative deformations of the Hilbert scheme as $t$ varies. (We similarly expect the center for $q$ torsion to be the pullback of this family through a suitable base change; this does not quite follow from the usual arguments, since those depended on strong flatness for the spherical algebra.) Such a family was constructed in [26] (see also [20] for the case of $\mathbb{P}^{2}$ ), and thus we naturally conjecture that they agree under a suitable reparametrization. The significance of this is that the 
construction of $[20,26]$ is as a moduli space of sheaves on a noncommutative surface (essentially $\operatorname{Proj} \mathcal{S}^{(1)}$ ), and there are some natural birational transformations between such moduli spaces. In particular, under certain conditions, the deformed Hilbert scheme is birational to a moduli space of sheaves with 1-dimensional support, which in turn may be interpreted as a moduli space of elliptic difference equations. We thus expect that our deformations are similarly closely related to noncommutative deformations of such moduli spaces.

When $q=t=0$ (i.e., the usual Hilbert scheme), this birational map may be described as follows. Let $X$ be a rational surface with a chosen anticanonical curve $C_{a}$, and let $D$ be a divisor class such that $\mathcal{O}_{C_{a}}(D)$ is trivial and the linear system $|D|$ is generically integral. The curves in this linear system have genus $g=D^{2} / 2+1$, and the linear system is itself a $\mathbb{P}^{g}$. There is a natural incidence relation between $\operatorname{Hilb}^{g}(X)$ and the linear system, i.e., whether the curve contains the $g$-point subscheme $Z$. Each divisor in $|D|$ is incident with a $g$-dimensional subscheme of $\operatorname{Hilb}^{g}(X)$, and thus Hilb $(X)$ is birational to the relative Hilb ${ }^{g}$ of the linear system. For $C \in|D|$, there is a natural map from $\operatorname{Hilb}^{g}(C)$ to a compactification of $\operatorname{Pic}^{g}(C)$ (i.e., torsionfree sheaves on $C$ ), and again this is generically invertible. In other words, the incidence relation is the graph of a birational map as desired.

There are two things that may go wrong with the map from $\operatorname{Hilb}^{g}(X)$ to the relative $\mathrm{Pic}^{g}$ : there may be more than one curve in the linear system containing the given subscheme, and the curve may be reducible (so that the resulting torsion-free sheaf may fail to be stable). The first issue happens when $h^{0}(\mathcal{I}(D))>1$ (where $\mathcal{I}$ is the ideal sheaf of $Z$ ); since this sheaf has Euler characteristic 0, we expect this to happen only in codimension $\geq 3$. (In fact, given a particular curve $C$ containing $Z$, the deformations of $C$ that still contain $Z$ are given by a subsheaf of the cotangent sheaf corresponding to $\mathcal{O}_{C}(K-Z)$, and thus $C$ is moveable iff $Z$ is moveable in $\mathrm{Pic}^{g}(C)$, so this is codimension $\geq 2$ in the graph of the birational map.) For generic parameters, the only reducible curves of $D$ will be those meeting $C_{a}$ and thus having it as a component. Since $\left|D-C_{a}\right| \cong \mathbb{P}^{g-1}$, there are two divisors in $\operatorname{Hilb}^{g}(X)$ compatible with such a reducible curve: either all $g$ points lie on a curve of $\left|D-C_{a}\right|$ or at least one point lies on $C_{a}$. (These correspond to the divisor classes on $\operatorname{Hilb}^{g}(X)$ denoted by $\delta+D-C_{a}$ and $C_{a}$ respectively relative to the above basis.) For an appropriate choice of stability conditions, the map is welldefined on the locus where one point lies on $C_{a}$, and contracts the other divisor to a subscheme of codimension $\geq 2$.

We thus find that if we remove the unique divisor of class $\delta+D-C_{a}$ from $\operatorname{Hilb}^{g}(X)$, then the result is not only birational to the compactified relative $\operatorname{Pic}^{g}(C)$, but the birational map is an isomorphism in codimension 1. The compactified relative $\operatorname{Pic}^{g}(C)$ is an abelian fibration over $\mathbb{P}^{g} \cong|D|$ (with integral fibers), and the derived autoequivalences of the fibers should extend to derived autoequivalences of $\operatorname{Pic}^{g}(C)$. One also expects that birational maps which are isomorphisms in codimension 1 should induce derived equivalences, and thus we expect that contracting the given divisor on $\operatorname{Hilb}^{g}(X)$ should give a projective scheme with a large family of derived autoequivalences. Moreover, since the noncommutative deformations of a scheme are functions of the derived category alone, we should expect these derived autoequivalences to act on the corresponding formal neighborhood in the family corresponding to $\mathcal{S}^{(n)}$, and thus one hopes on the family itself. The result would be an analogue of the derived equivalences of geometric Langlands, with (symmetric) elliptic difference equations replacing connections. (For $n=1$, such derived equivalences in fact exist, see [26, Section 12].)

One part of the above line of reasoning is the strong suggestion that the divisor $\delta+D-C_{a}$ should be contractible. Something along these lines holds for $\mathcal{S}$.

Proposition 9.6. For any $v \in\left\langle\delta, s, f, e_{1}, \ldots, e_{m}\right\rangle$, the union

$$
\bigcup_{k \in \mathbb{Z}} \mathcal{S}_{\eta^{\prime}, x_{1}, \ldots, x_{m} ; q, t}^{(n)}(0, v+k(\delta+(n-1) f))
$$


is coherent and equal to

$$
\begin{aligned}
\mathcal{S}_{\eta^{\prime}, x_{1}, \ldots, x_{m} ; q, t}^{(n)} & (0, v+(d-a)(\delta+(n-1) f)) \\
& =\mathcal{S}_{\eta^{\prime}+(n-1) t, x_{1}, \ldots, x_{m} ; q, 0}^{(n)}(0, v+(d-a)(\delta+(n-1) f))
\end{aligned}
$$

if $v=a \delta+d s+\cdots$.

Proof. Indeed, this is a nested sequence of sheaf bimodules as $k$ increases, and once $k \geq d-a$, there are no longer any $t$-dependent vanishing conditions, and thus the sequence stabilizes. The only remaining dependence on $t$ is in the twisting datum, and thus we may make $t=0$ as long as we adjust $\eta^{\prime}$ accordingly.

Remark. For the commutative Hilbert scheme, what this is suggesting is that if $D$ is the unique divisor of class $\delta+(n-1) f$, then for any line bundle $\mathcal{L}, \Gamma\left(\operatorname{Hilb}^{n}(X) \backslash D ; \mathcal{L}\right)$ is finite-dimensional (and isomorphic to the space of global sections of some $\mathcal{L}^{\prime}$ ). Applying this to powers of an ample bundle gives a graded algebra the Proj of which has the same homogeneous coordinate ring as $\operatorname{Hilb}^{n}(X) \backslash D$, and thus the corresponding map contracts $D$ to a subscheme of codimension $\geq 2$. Note that we cannot expect this to be a blow down, but it should be similar: if we take $e_{m}$ rather than $\delta+(n-1) f$, then the same construction gives the Hilbert scheme of the blow down of $X$.

Remark. The case $D-C_{a}=(n-1) f$ (and thus $\left.m=2 n+6\right)$ corresponds to a moduli space of second-order equations with $2 n+6$ singularities.

As this holds even without the constraint that $D \cdot C_{a}=0$, it seems likely that something analogous should hold for any divisor. To be precise, if $w \in \mathbb{Z}\left\langle s, f, e_{1}, \ldots, e_{m}\right\rangle$ is such that $\mathcal{O}_{X_{m}}(w)$ is generically acyclic with $n$ global sections, then we conjecture that the union

$$
\bigcup_{k \in \mathbb{Z}} \mathcal{S}_{\eta^{\prime}, x_{1}, \ldots, x_{m} ; q, t}^{(n)}(0, v+k(\delta+w))
$$

stabilizes (and is thus coherent) for large $k$, and moreover that the smallest $k$ for which it stabilizes is a linear function of $v$, so that the resulting category may be identified with a subcategory of $\mathcal{S}$. The result would then be invariant under the shift in parameters corresponding to $\delta+w$, and thus equivalent to a category with $t=0$. Something along these lines appears to work for $n=2$ with $w=2 s+2 f-e_{1}-\cdots-e_{7}$, in that the Euler characteristic of the line bundle reaches a maximum at $k$ depending linearly on $v$. (This case corresponds to the unique other case (modulo the Fourier transform and elementary transformations) of a divisor $D$ such that $\left(D+C_{a}\right) \cap C_{a}=\varnothing$ and $\left(D+C_{a}\right)^{2}=2$, corresponding to a moduli space of line bundles on genus 2 curves.)

\section{Acknowledgements}

The author would particularly like to thank P. Etingof both for asking the original seed question (with an important assist from A. Okounkov!) and hosting the author's sabbatical at MIT (which the author would also like to thank, naturally) where much of the basic approach was worked out, with great assistance from conversations with not only Etingof but also (regarding various geometrical issues) B. Poonen. Thanks also go to T. Graber and E. Mantovan for helpful and encouraging conversations regarding the constructions of Section 2 as well as various general algebraic geometric questions, and to O. Chalykh for asking some fruitful questions about residue conditions. The author's work presented here was supported in part by grants from the National Science Foundation, DMS-1001645 and DMS-1500806. 


\section{References}

[1] Artin M., Tate J., Van den Bergh M., Some algebras associated to automorphisms of elliptic curves, in The Grothendieck Festschrift, Vol. I, Progr. Math., Vol. 86, Editors P. Cartier, L. Illusie, N.M. Katz, G. Laumon, Yu.I. Manin, Birkhäuser Boston, Boston, MA, 2007, 33-85.

[2] Artin M., Van den Bergh M., Twisted homogeneous coordinate rings, J. Algebra 133 (1990), 249-271.

[3] Bondal A.I., Polishchuk A.E., Homological properties of associative algebras: the method of helices, Izv. Math. 42 (1994), 219-260.

[4] Brion M., Stable properties of plethysm: on two conjectures of Foulkes, Manuscripta Math. 80 (1993), 347-371.

[5] Conway J.H., Sloane N.J.A., Low-dimensional lattices. II. Subgroups of GL(n, Z), Proc. Roy. Soc. London Ser. A 419 (1988), 29-68.

[6] van Diejen J.F., Integrability of difference Calogero-Moser systems, J. Math. Phys. 35 (1994), $2983-3004$.

[7] van Diejen J.F., Difference Calogero-Moser systems and finite Toda chains, J. Math. Phys. 36 (1995), 1299-1323.

[8] Eichler M., Zagier D., The theory of Jacobi forms, Progress in Mathematics, Vol. 55, Birkhäuser Boston, Inc., Boston, MA, 1985.

[9] Ellingsrud G., Göttsche L., Lehn M., On the cobordism class of the Hilbert scheme of a surface, J. Algebraic Geom. 10 (2001), 81-100, arXiv:math.AG/9904095.

[10] Fulton W., Olsson M., The Picard group of $\mathcal{M}_{1,1}$, Algebra Number Theory 4 (2010), 87-104, arXiv:0704.2214.

[11] Ginzburg V., Kapranov M., Vasserot E., Residue construction of Hecke algebras, Adv. Math. 128 (1997), 1-19, arXiv:alg-geom/9512017.

[12] Gordon I., Stafford J.T., Rational Cherednik algebras and Hilbert schemes, Adv. Math. 198 (2005), 222-274, arXiv:math.RA/0407516.

[13] Gritsenko V., Modular forms and moduli spaces of abelian and K3 surfaces, St. Petersburg Math. J. 6 (1995), 1179-1208.

[14] Gritsenko V.A., Fourier-Jacobi functions in $n$ variables, J. Soviet Math. 53 (1991), 243-252.

[15] Katz N.M., Mazur B., Arithmetic moduli of elliptic curves, Annals of Mathematics Studies, Vol. 108, Princeton University Press, Princeton, NJ, 1985.

[16] Komori Y., Hikami K., Quantum integrability of the generalized elliptic Ruijsenaars models, J. Phys. A: Math. Gen. 30 (1997), 4341-4364.

[17] Krieg A., Jacobi forms of several variables and the Maaßspace, J. Number Theory 56 (1996), 242-255.

[18] Looijenga E., Root systems and elliptic curves, Invent. Math. 38 (1976), 17-32.

[19] Lusztig G., Singularities, character formulas, and a $q$-analog of weight multiplicities, in Analysis and Topology on Singular Spaces, II, III (Luminy, 1981), Astérisque, Vol. 101, Soc. Math. France, Paris, 1983, 208-229.

[20] Nevins T.A., Stafford J.T., Sklyanin algebras and Hilbert schemes of points, Adv. Math. 210 (2007), 405-478, arXiv:math.AG/0310045.

[21] Oblomkov A., Double affine Hecke algebras of rank 1 and affine cubic surfaces, Int. Math. Res. Not. 2004 (2004), 877-912, arXiv:math.RT/0306393.

[22] Polishchuk A., Abelian varieties, theta functions and the Fourier transform, Cambridge Tracts in Mathematics, Vol. 153, Cambridge University Press, Cambridge, 2003.

[23] Rains E.M., $B C_{n}$-symmetric Abelian functions, Duke Math. J. $135 \quad$ (2006), 99-180, arXiv:math.CO/0402113.

[24] Rains E.M., Transformations of elliptic hypergeometric integrals, Ann. of Math. 171 (2010), 169-243, arXiv:math.QA/0309252.

[25] Rains E.M., Generalized Hitchin systems on rational surfaces, arXiv:1307.4033.

[26] Rains E.M., The noncommutative geometry of elliptic difference equations, arXiv:1607.08876.

[27] Rains E.M., Multivariate quadratic transformations and the interpolation kernel, SIGMA 14 (2018), 019, 69 pages, arXiv:1408.0305. 
[28] Rains E.M., Ruijsenaars S., Difference operators of Sklyanin and van Diejen type, Comm. Math. Phys. 320 (2013), 851-889, arXiv:1203.0042.

[29] Rosengren H., Elliptic hypergeometric functions, in Lectures on Orthogonal Polynomials and Special Functions, Cambridge University Press, Cambridge, to appear, arXiv:1608.06161.

[30] Ruijsenaars S.N.M., First order analytic difference equations and integrable quantum systems, J. Math. Phys. 38 (1997), 1069-1146.

[31] Saito K., Extended affine root systems. II. Flat invariants, Publ. Res. Inst. Math. Sci. 26 (1990), 15-78.

[32] Sekiguchi T., On projective normality of Abelian varieties. II, J. Math. Soc. Japan 29 (1977), $709-727$.

[33] Shioda T., On elliptic modular surfaces, J. Math. Soc. Japan 24 (1972), 20-59.

[34] Silverman J.H., The arithmetic of elliptic curves, 2nd ed., Graduate Texts in Mathematics, Vol. 106, Springer, Dordrecht, 2009.

[35] Spiridonov V.P., Warnaar S.O., Inversions of integral operators and elliptic beta integrals on root systems, Adv. Math. 207 (2006), 91-132, arXiv:math.CA/0411044.

[36] Stembridge J.R., Tight quotients and double quotients in the Bruhat order, Electron. J. Combin. 11 (2005), 14, 41 pages.

[37] Van den Bergh M., A translation principle for the four-dimensional Sklyanin algebras, J. Algebra 184 (1996), 435-490.

[38] Van den Bergh M., Non-commutative $\mathbb{P}^{1}$-bundles over commutative schemes, Trans. Amer. Math. Soc. 364 (2012), 6279-6313, arXiv:math.RA/0102005. 